\title{
ON THE FREDHOLM AND UNIQUE SOLVABILITY OF NONLOCAL ELLIPTIC PROBLEMS IN MULTIDIMENSIONAL DOMAINS
}

\author{
P. L. GUREVICH AND A. L. SKUBACHEVSKII
}

\section{INTRODUCTION}

We consider elliptic equations of order $2 m$ in a bounded domain $Q \subset \mathbb{R}^{n}$ with nonlocal boundary-value conditions connecting the values of a solution and its derivatives on $(n-1)$-dimensional smooth manifolds $\Gamma_{i}$ with the values on manifolds $\omega_{i}\left(\Gamma_{i}\right)$, where $\bigcup_{i} \overline{\Gamma_{i}}=\partial Q$ is a boundary of $Q$ and $\omega_{i}$ are $C^{\infty}$ diffeomorphisms. The presence of nonlocal terms leads to the appearance of power-law singularities of solutions and their derivatives at the points of the set $\mathcal{K}_{1}=\bigcup_{i}\left(\overline{\Gamma_{i}} \backslash \Gamma_{i}\right)$ (which is called the set of conjugation points). Therefore, nonlocal elliptic problems are naturally studied in weighted spaces $H_{a}^{l+2 m}(Q), a \in \mathbb{R}, l \geq 0$ is an integer (see definition (1.5)), originally introduced in the theory of elliptic problems in nonsmooth domains [15]. Because of the transformations $\omega_{i}$ occurring in nonlocal terms, the points of the set $\mathcal{K}_{1}$ turn out to be connected with the points of the set

$$
\left\{\bigcup_{i} \omega_{i}\left(\mathcal{K}_{1}\right)\right\} \cup\left\{\bigcup_{i, j} \omega_{j}\left(\omega_{i}\left(\mathcal{K}_{1}\right) \cap \Gamma_{j}\right)\right\} .
$$

The latter points belong to $Q$ or $\partial Q$. Therefore, we must consider certain consistency conditions at the points of the set

$$
\mathcal{K}=\mathcal{K}_{1} \cup\left\{\bigcup_{i} \omega_{i}\left(\mathcal{K}_{1}\right)\right\} \cup\left\{\bigcup_{i, j} \omega_{j}\left(\omega_{i}\left(\mathcal{K}_{1}\right) \cap \Gamma_{j}\right)\right\} .
$$

The following two approaches are possible. First, one can consider all the points of the set $\mathcal{K}$ as singular points in the definition of weighted spaces, which allows one to study nonlocal elliptic problems for any value of the parameter $a \in \mathbb{R}$ (see 24] for the case $n=2$ ). Second, one can assume that only the points of the set $\mathcal{K}_{1}$ or the set $\mathcal{K} \cap \partial Q$ are singular points in the definition of weighted spaces, which allows one to study nonlocal elliptic problems only for $a>l+2 m-1$ (see [26, 27]).

It is proved in [20, 22, that "local" elliptic problems in bounded domains have the Fredholm property (see Definition 2.1) if some model elliptic operators (depending on a parameter $\omega$ ) in plane angles have a trivial kernel and cokernel for all $\omega \in S^{n-3}$, where

$$
S^{n-3}=\left\{\omega \in \mathbb{R}^{n-2}:|\omega|=1\right\} .
$$

Similarly, elliptic operators in $\mathbb{R}^{2} \backslash\{0\}$ with the parameter $\omega \in S^{n-3}$ arise if the points of the set $\mathcal{K} \cap Q$ are considered as singular points in the definition of weighted spaces.

2000 Mathematics Subject Classification. Primary 35J40.

Key words and phrases. Elliptic problems, nonlocal boundary conditions, solvability, nonsmooth domains.

Supported by the Russian Foundation for Basic Research (project No. 04-01-00256) and the Russian President Grant (project No. MK-980.2005.1). The first author was also supported by the Alexander von Humboldt Foundation. 
However, we prove in this paper that these operators are not isomorphisms; see Sec. 3 . Therefore, unlike the case of plane domains, if $n \geq 3$ and $\mathcal{K} \cap Q \neq \varnothing$, only the points of the set $\mathcal{K}_{1}$ or the set $\mathcal{K} \cap \partial Q$ can be considered as singular points. This leads to the restriction $a>l+2 m-1$ (see Sec. 4.1 for details).

The paper is organized as follows.

The setting of nonlocal elliptic problems is presented in Sec. 1. In the same section, we define model problems in dihedral angles and introduce function spaces. In Sec. 2, we consider the solvability of nonlocal problems in dihedral angles. In particular, we give an example of a nonlocal elliptic problem in a dihedral angle which is uniquely solvable on weighted spaces for $0 \leq a \leq 2$. In Sec. 3 . we show that an elliptic operator of order $2 m$ acting from $H_{a}^{l+2 m}\left(\mathbb{R}^{n}\right)$ to $H_{a}^{l}\left(\mathbb{R}^{n}\right)$ is not an isomorphism for any $a \in \mathbb{R}$ and integer $l \geq 0$. Here we suppose that the points of the set

$$
\mathcal{P}=\left\{x=(y, z) \in \mathbb{R}^{n}: y=0, z \in \mathbb{R}^{n-2}\right\}
$$

are singular in the definition of weighted spaces $H_{a}^{l+2 m}\left(\mathbb{R}^{n}\right)$ and $H_{a}^{l}\left(\mathbb{R}^{n}\right)$. In Sec. 4, we prove a priori estimates for solutions of nonlocal problems in bounded domains. In Sec. 5. we construct a right regularizer for those problems. Thus, we prove a theorem on the Fredholm solvability of nonlocal elliptic problems in bounded domains. Section 6 is devoted to generalizations of nonlocal elliptic problems to the case of nonlocal terms supported on the manifolds $\omega_{i s}\left(\Gamma_{i}\right)$ near the set $\mathcal{K}_{1}$ and abstract nonlocal terms supported outside the set $\mathcal{K}_{1}$. In Secs. 7 and 8 , we prove the unique solvability of nonlocal elliptic problems with a parameter.

Note that it was A. V. Bitsadze and A. A. Samarskiu [3] who first considered an elliptic equation with nonlocal conditions imposed on the shifts of different parts of the boundary of a rectangle. In the general situation, they formulated this problem as an unsolved problem. Solvability and regularity of solutions for higher-order elliptic equations with general nonlocal conditions supported near the boundary were studied in 24, 25, 26, 27. Second-order elliptic equations with nonlocal conditions near the boundary were also considered in [14, 12, 11. One can find various applications of nonlocal elliptic problems as well as a comprehensive bibliography of the question in [24, 27, 28, 8].

\section{Setting OF NONLOCAL ELLIPTIC PROBLEMS}

1.1. Let $Q \subset \mathbb{R}^{n}(n \geq 3)$ be a bounded domain with boundary $\partial Q=\bigcup_{i=1}^{N_{0}} \overline{\Gamma_{i}}$, where $\Gamma_{i}$ are open connected, in the topology of $\partial Q,(n-1)$-dimensional $C^{\infty}$ manifolds. Assume that, in a neighborhood of each point $g \in \overline{\Gamma_{i}} \backslash \Gamma_{i}$, the domain $Q$ is diffeomorphic to an $n$-dimensional dihedral angle

$$
\Theta=\left\{x=(y, z) \in \mathbb{R}^{n}: d_{1}<\varphi<d_{2}, z \in \mathbb{R}^{n-2}\right\},
$$

where $r, \varphi$ are the polar coordinates of the point $y \in \mathbb{R}^{2}, d_{j}=d_{j}(g), j=1,2$.

Introduce the differential operators

$$
A(x, D)=\sum_{|\alpha| \leq 2 m} a_{\alpha}(x) D^{\alpha}, \quad B_{i \mu s}(x, D)=\sum_{|\alpha| \leq m_{i \mu}} b_{i \mu s \alpha}(x) D^{\alpha},
$$

where $a_{\alpha}, b_{i \mu s \alpha} \in C^{\infty}\left(\mathbb{R}^{n}\right)$ are complex-valued functions $\left(i=1, \ldots, N_{0} ; \mu=1, \ldots, m\right.$; $\left.s=0, \ldots, S_{i}\right), m_{i \mu} \leq 2 m-1, \alpha=\left(\alpha_{1}, \ldots, \alpha_{n}\right),|\alpha|=\alpha_{1}+\cdots+\alpha_{n}, D^{\alpha}=D_{1}^{\alpha_{1}} \ldots D_{n}^{\alpha_{n}}$, $D_{j}=-i \partial / \partial x_{j}$. If it is necessary to indicate the variables with respect to which a function $u$ is differentiated, we write $D_{y}^{\alpha} u, D_{z}^{\alpha} u$, etc.

Let $\omega_{i s}\left(i=1, \ldots, N_{0} ; s=1, \ldots, S_{i}\right)$ denote a $C^{\infty}$ diffeomorphism mapping some neighborhood $\Omega_{i}$ of the manifold $\Gamma_{i}$ onto the set $\omega_{i s}\left(\Omega_{i}\right)$ in such a way that $\omega_{i s}\left(\Gamma_{i}\right) \subset Q$. 
Assume that the set

$$
\mathcal{K}=\left\{\bigcup_{i}\left(\overline{\Gamma_{i}} \backslash \Gamma_{i}\right)\right\} \cup\left\{\bigcup_{i, s} \omega_{i s}\left(\overline{\Gamma_{i}} \backslash \Gamma_{i}\right)\right\} \cup\left\{\bigcup_{j, p} \bigcup_{i, s} \omega_{j p}\left(\omega_{i s}\left(\overline{\Gamma_{i}} \backslash \Gamma_{i}\right) \cap \Gamma_{j}\right)\right\}
$$

can be represented as follows:

$$
\mathcal{K}=\mathcal{K}_{1} \cup \mathcal{K}_{2} \cup \mathcal{K}_{3},
$$

where $\mathcal{K}_{1}=\bigcup_{\nu} \mathcal{K}_{1 \nu}=\partial Q \backslash \bigcup_{i} \Gamma_{i}, \mathcal{K}_{2}=\bigcup_{\nu} \mathcal{K}_{2 \nu} \subset \bigcup_{i} \Gamma_{i}, \mathcal{K}_{3}=\bigcup_{\nu} \mathcal{K}_{3 \nu} \subset Q(\nu=$ $\left.1, \ldots, N_{j} ; j=1,2,3\right), \mathcal{K}_{j \nu}$ are mutually disjoint $(n-2)$-dimensional connected $C^{\infty}$ manifolds without a boundary. In particular, the sets $\mathcal{K}_{2}$ and $\mathcal{K}_{3}$ may be empty.

We study the following nonlocal problem:

$$
\begin{gathered}
A u \equiv A(x, D) u(x)=f_{0}(x), \quad x \in Q, \\
\left.B_{i \mu} u \equiv \sum_{s=0}^{S_{i}}\left(B_{i \mu s}(x, D) u\right)\left(\omega_{i s}(x)\right)\right|_{\Gamma_{i}}=f_{i \mu}(x), \\
x \in \Gamma_{i} ; i=1, \ldots, N_{0} ; \mu=1, \ldots, m,
\end{gathered}
$$

where $\left.\left(B_{i \mu s}(x, D) u\right)\left(\omega_{i s}(x)\right)\right|_{\Gamma_{i}}=\left.B_{i \mu s}(\hat{x}, D) u(\hat{x})\right|_{\hat{x}=\omega_{i s}(x), x \in \Gamma_{i}}, \omega_{i 0}(x) \equiv x$.

We assume throughout that the operators $A(x, D)$ and $B_{i \mu 0}(x, D)$ satisfy the following conditions.

Condition 1.1. The operator $A(x, D)$ is properly elliptic for each $x \in \bar{Q}$.

Condition 1.2. The system $\left\{B_{i \mu 0}(x, D)\right\}_{\mu=1}^{m}$ satisfies the Lopatinskii condition with respect to $A(x, D)$ and is normal for all $i=1, \ldots, N_{0}$ and $x \in \overline{\Gamma_{i}}$ (see [19, Chap. 2, Sec. 1.4]).

Denote by $\omega_{i s}^{+1}$ the transformation $\omega_{i s}: \Omega_{i} \rightarrow \omega_{i s}\left(\Omega_{i}\right)$ and by $\omega_{i s}^{-1}: \omega_{i s}\left(\Omega_{i}\right) \rightarrow \Omega_{i}$ the inverse transformation.

Definition 1.1. The set of all points that can be obtained from a point $g \in \mathcal{K}_{1}$ by consecutively applying to it the transformations $\omega_{i s}^{+1}$ or $\omega_{i s}^{-1}$ mapping the points of the set $\mathcal{K}_{1}$ to $\mathcal{K}_{1}$ is called an orbit of the point $g$ and is denoted by $\mathcal{O}(g)$.

In other words, the orbit $\mathcal{O}(g)$ consists of the point $g \in \mathcal{K}_{1}$ and the points that can be obtained from $g$ in the following way: given a point $h \in \mathcal{O}(g)$, the point $\omega_{i s}(h)$ belongs to $\mathcal{O}(g)$ iff $h \in \overline{\Gamma_{i}} \cap \mathcal{K}_{1}$ and $\omega_{i s}(h) \in \mathcal{K}_{1}$, while the point $\omega_{i s}^{-1}(h)$ belongs to $\mathcal{O}(g)$ iff $h \in \omega_{i s}\left(\overline{\Gamma_{i}}\right) \cap \mathcal{K}_{1}$.

Assume that the following condition holds.

Condition 1.3 (Finiteness of the orbits). (1) For each point $g \in \mathcal{K}_{1}$, the orbit $\mathcal{O}(g)$ consists of finitely many points $g_{j}, j=1, \ldots, N=N(g)$.

(2) There are neighborhoods $\hat{V}\left(g_{j}\right) \subset V\left(g_{j}\right) \subset \mathbb{R}^{n} \backslash\left(\mathcal{K}_{2} \cup \mathcal{K}_{3}\right), V\left(g_{j}\right) \cap V\left(g_{p}\right)=\varnothing$ $(j \neq p)$, of the points $g_{j} \in \mathcal{O}(g)$ such that, if $g_{j} \in \overline{\Gamma_{i}}$ and $\omega_{i s}\left(g_{j}\right)=g_{p}$, then $\hat{V}\left(g_{j}\right) \subset \Omega_{i}$ and $\omega_{i s}\left(\hat{V}\left(g_{j}\right)\right) \subset V\left(g_{p}\right)$.

The following condition means that the support of nonlocal terms intersects the boundary at the points of the set $\mathcal{K}_{1}$ in a nontangential way.

Condition 1.4 (Nontangential approach). For each point $g \in \mathcal{K}_{1}$ and $j=1, \ldots, N(g)$, there exists a smooth nondegenerate change of variables $x \rightarrow x^{\prime}=x^{\prime}(g, j)$ such that the 
neighborhood $V\left(g_{j}\right)$ reduces, under this change of variables, to some neighborhood of the origin $V(0)$ and, moreover:

(1) The sets $Q \cap V\left(g_{j}\right)$ and $\Gamma_{i} \cap V\left(g_{j}\right)$ reduce to the intersection of a dihedral angle $\Theta_{j}$ with $V(0)$ and to the intersection of a side $\Gamma_{j \rho}(\rho=1$ or $\rho=2)$ of $\Theta_{j}$ with $V(0)$, respectively;

(2) Each transformation $\omega_{i s}(x)$, for $x \in \hat{V}\left(g_{j}\right), g_{j} \in \overline{\Gamma_{i}}$, reduces to the composition of rotation and homothety on the plane $\left\{y^{\prime}\right\}$ in the new variables $x^{\prime}=\left(y^{\prime}, z^{\prime}\right)$, where $y^{\prime} \in \mathbb{R}^{2}$ and $z^{\prime} \in \mathbb{R}^{n-2}$.

Remark 1.1. If $N(g)=1$, then the orbit of $g \in \mathcal{K}_{1}$ consists of the unique point $g$. This is the case iff the following two conditions are fulfilled:

(1) $\omega_{i s}(g)=g$ for all $i$ and $s$ such that $g \in \overline{\Gamma_{i}} \cap \mathcal{K}_{1}$ and $\omega_{i s}(g) \in \mathcal{K}_{1}$;

(2) there do not exist indices $i$ and $s$ and a point $h \in \overline{\Gamma_{i}} \cap \mathcal{K}_{1}$ such that $h \neq g$ and $\omega_{i s}(h)=g$.

1.2. For any domain $\Omega$, denote by $W^{k}(\Omega)=W_{2}^{k}(\Omega)(k \geq 0$ is an integer) the Sobolev space. Denote by $W^{k-1 / 2}(\Gamma)(k \geq 1$ is an integer $)$ the space of traces on a smooth $(n-1)$-dimensional manifold $\Gamma \subset \bar{\Omega}$, with the norm

$$
\|\psi\|_{W^{k-1 / 2}(\Gamma)}=\inf \|v\|_{W^{k}(\Omega)} \quad\left(v \in W^{k}(\Omega):\left.v\right|_{\Gamma}=\psi\right) .
$$

If $X$ is a domain in $\mathbb{R}^{n}, n=1,2, \ldots$, we denote by $C_{0}^{\infty}(X)$ the set of functions infinitely differentiable on $\bar{X}$ and compactly supported on $X$. If $M$ is a union of finitely many $(n-l)$-dimensional manifolds $(l=1, \ldots, n)$ lying in $\bar{X}$, we denote by $C_{0}^{\infty}(\bar{X} \backslash M)$ the set of functions infinitely differentiable on $\bar{X}$ and compactly supported on $\bar{X} \backslash M$.

Now we introduce different weighted spaces for different domains $\Omega$. Consider the following cases:

(1) $\Omega=Q$; denote either $K=\mathcal{K}_{1}$ or $K=\mathcal{K}_{1} \cup \mathcal{K}_{2}$ (cf. Condition 4.1 in Sec. 44), and let $\rho(x)$ be a function such that $\rho \in C^{\infty}\left(\mathbb{R}^{n} \backslash K\right), \rho(x)>0$ for $x \in \mathbb{R}^{n} \backslash K$ and it is equivalent, in a neighborhood of the set $K$, to the distance from a point $x \in \Omega$ to the set $K$;

(2) $\Omega \subset \mathbb{R}^{n}$ is a bounded domain with boundary $\partial \Omega \in C^{\infty}$; denote by $K$ some $(n-2)$-dimensional manifold of class $C^{\infty}$ lying in $\bar{\Omega}$, and let $\rho$ be the same function as in case 1 ;

(3) $\Omega$ is an $n$-dimensional dihedral angle $\Theta$; denote $K=\mathcal{P}$, where

$$
\mathcal{P}=\left\{x=(y, z) \in \mathbb{R}^{n}: y=0, z \in \mathbb{R}^{n-2}\right\},
$$

and let $\rho(x)=|y|$

(4) $\Omega=\mathbb{R}^{n}$; denote $K=\mathcal{P}$, and let $\rho(x)=|y|$.

Introduce the weighted space $H_{a}^{k}(\Omega)=H_{a}^{k}(\Omega, K)$ as the completion of the set $C_{0}^{\infty}(\bar{\Omega} \backslash K)$ with respect to the norm

$$
\|u\|_{H_{a}^{k}(\Omega)}=\left(\sum_{|\alpha| \leq k} \int_{\Omega} \rho^{2(a-k+|\alpha|)}\left|D^{\alpha} u\right|^{2} d x\right)^{1 / 2},
$$

where $k \geq 0$ is an integer and $a \in \mathbb{R}$.

Denote by $H_{a}^{k-1 / 2}(\Gamma)(k \geq 1$ is an integer $)$ the space of traces on a smooth $(n-1)$ dimensional manifold $\Gamma \subset \bar{\Omega}$, with the norm

$$
\|\psi\|_{H_{a}^{k-1 / 2}(\Gamma)}=\inf \|v\|_{H_{a}^{k}(\Omega)} \quad\left(v \in H_{a}^{k}(\Omega):\left.v\right|_{\Gamma}=\psi\right) .
$$

One can similarly introduce the weighted spaces $H_{a}^{k}(\Omega)$ and $H_{a}^{k-1 / 2}(\Gamma)$ for $n=2$. In particular, we set $K=\{0\}$ for $\Omega=\theta=\left\{y \in \mathbb{R}^{2}: d_{1}<\varphi<d_{2}, 0<r\right\}$ or $\Omega=\mathbb{R}^{2}$. 
In what follows, we assume that $u \in H_{a}^{l+2 m}(Q)$ and $f=\left\{f_{0}, f_{i \mu}\right\} \in \mathcal{H}_{a}^{l}(Q, \Gamma)$ in problem (1.3), (1.4), where

$$
\mathcal{H}_{a}^{l}(Q, \Gamma)=H_{a}^{l}(Q) \times \prod_{i, \mu} H_{a}^{l+2 m-m_{i \mu}-1 / 2}\left(\Gamma_{i}\right)
$$

and $l \geq 0$ is an integer.

1.3. Fix an arbitrary point $g \in \mathcal{K}_{1}$. By Condition 1.3 , the orbit $\mathcal{O}(g)$ consists of finitely many points $g_{j}, j=1, \ldots, N=N(g)$. We now reduce problem (1.3), (1.4) to a system of $N$ elliptic equations in dihedral angles with nonlocal boundary-value conditions. To do this, we suppose that

$$
\operatorname{supp} u \subset\left(\bigcup_{j} \hat{V}\left(g_{j}\right)\right) \cap \bar{Q} .
$$

Denote by $u_{j}(x)$ the function $u(x)$ for $x \in Q \cap V\left(g_{j}\right)$. If $g_{j} \in \overline{\Gamma_{i}}$ and $x \in \hat{V}\left(g_{j}\right)$, then $\omega_{i s}(x) \in V\left(g_{p}\right)$ for some $p, 1 \leq p \leq N$, by Condition 1.3. Denote the function $u\left(\omega_{i s}(x)\right)$ by $u_{p}\left(\omega_{i s}(x)\right)$. It is clear that $u\left(\omega_{i 0}(x)\right)=u(x)=u_{j}(x)$. In the above notation, problem (1.3), (1.4) takes the form

$$
\begin{gathered}
A(x, D) u_{j}(x)=f_{0}(x), \quad x \in Q \cap \hat{V}\left(g_{j}\right) ; \\
\left.\sum_{s \in S_{i j}^{g}}\left(B_{i \mu s}(x, D) u_{p}\right)\left(\omega_{i s}(x)\right)\right|_{\Gamma_{i}}=f_{i \mu}(x), \quad x \in \hat{V}\left(g_{j}\right) \cap \Gamma_{i} ; \\
i \in\left\{1 \leq i \leq N_{0}: \hat{V}\left(g_{j}\right) \cap \Gamma_{i} \neq \varnothing\right\} ; j=1, \ldots, N ; \mu=1, \ldots, m,
\end{gathered}
$$

where $S_{i j}^{g}=\left\{0 \leq s \leq S_{i}: \omega_{i s}\left(g_{j}\right)=g_{p} \in \mathcal{O}(g)\right.$ for some $\left.p=1, \ldots, N\right\}$.

Using the change of variables $x \rightarrow x^{\prime}(g, j)$ from Sec. 1.1. we introduce the functions $v_{j}\left(x^{\prime}\right)=u_{j}\left(x\left(x^{\prime}\right)\right)$. By Condition 1.4 problem (1.7), (1.8) takes the following form:

$$
\begin{array}{r}
A_{j}\left(x^{\prime}, D_{y^{\prime}}, D_{z^{\prime}}\right) v_{j}\left(x^{\prime}\right)=f_{j}\left(x^{\prime}\right), \quad x^{\prime} \in \Theta_{j}, j=1, \ldots, N ; \\
\left.\sum_{k=1}^{N} \sum_{s=0}^{S_{j \rho k}}\left(B_{j \rho \mu k s}\left(x^{\prime}, D_{y^{\prime}}, D_{z^{\prime}}\right) v_{k}\right)\left(\mathcal{G}_{j \rho k s} y^{\prime}, z^{\prime}\right)\right|_{\Gamma_{j \rho}}=f_{j \rho \mu}\left(x^{\prime}\right), \\
x^{\prime} \in \Gamma_{j \rho} ; j=1, \ldots, N ; \rho=1,2 ; \mu=1, \ldots, m .
\end{array}
$$

Here the operators $A_{j}$ and $B_{j \rho \mu k s}$ have variable coefficients of class $C^{\infty}$;

$$
\begin{aligned}
\Theta_{j} & =\left\{x^{\prime}=\left(y^{\prime}, z^{\prime}\right): 0<d_{j 1}<\varphi<d_{j 2}, z^{\prime} \in \mathbb{R}^{n-2}\right\}, \\
\Gamma_{j \rho} & =\left\{x^{\prime}=\left(y^{\prime}, z^{\prime}\right): \varphi=d_{j \rho}, z^{\prime} \in \mathbb{R}^{n-2}\right\} ;
\end{aligned}
$$

$\mathcal{G}_{j \rho k s}$ is the operator of rotation by an angle $\varphi_{j \rho k s}$ and homothety with a coefficient $\chi_{j \rho k s}$ in the $y^{\prime}$-plane so that $d_{k 1}<d_{j \rho}+\varphi_{j \rho k s}<d_{k 2}$ and $0<\chi_{j \rho k s}$ for $(k, s) \neq(j, 0)$, while $\varphi_{j \rho j 0}=0$ and $\chi_{j \rho j 0}=1$ (i.e., $\left.\mathcal{G}_{j \rho j 0} y^{\prime} \equiv y^{\prime}\right) ; v=\left(v_{1}, \ldots, v_{N}\right)$.

Remark 1.2. If $g \in \overline{\Gamma_{i}}, N=N(g)=1$ (cf. Remark 1.1), and $\omega_{i s}(g) \neq g$ for all $s=1, \ldots, S_{i}$, then model problem (1.9), (1.10) contains no nonlocal terms due to the fact that the manifolds $\mathcal{K}_{j \nu}$ are mutually disjoint.

Introduce the following spaces of vector-valued functions:

$$
\mathcal{H}_{a}^{k}(\Theta)=\prod_{j=1}^{N} H_{a}^{k}\left(\Theta_{j}\right), \quad \mathcal{H}_{a}^{l}(\Theta, \Gamma)=\mathcal{H}_{a}^{l}(\Theta) \times \prod_{j=1}^{N} \prod_{\rho=1,2} \prod_{\mu=1}^{m} H_{a}^{l+2 m-m_{j \rho \mu}-1 / 2}\left(\Gamma_{j \rho}\right),
$$

where $m_{j \rho \mu}$ is the order of the operator $B_{j \rho \mu k s}\left(x^{\prime}, D_{y^{\prime}}, D_{z^{\prime}}\right)$. 
Consider the linear bounded operator $\mathcal{L}_{g}: \mathcal{H}_{a}^{l+2 m}(\Theta) \rightarrow \mathcal{H}_{a}^{l}(\Theta, \Gamma)$ given by

$$
\mathcal{L}_{g} v=\left\{A_{j}\left(D_{y^{\prime}}, D_{z^{\prime}}\right) v_{j}\left(y^{\prime}, z^{\prime}\right),\left.\sum_{k=1}^{N} \sum_{s=0}^{S_{j \rho k}}\left(B_{j \rho \mu k s}\left(D_{y^{\prime}}, D_{z^{\prime}}\right) v_{k}\right)\left(\mathcal{G}_{j \rho k s} y^{\prime}, z^{\prime}\right)\right|_{\Gamma_{j \rho}}\right\}
$$

where $A_{j}\left(D_{y^{\prime}}, D_{z^{\prime}}\right)$ and $B_{j \rho \mu k s}\left(D_{y^{\prime}}, D_{z^{\prime}}\right)$ are principal homogeneous parts of the operators $A_{j}\left(0, D_{y^{\prime}}, D_{z^{\prime}}\right)$ and $B_{j \rho \mu k s}\left(0, D_{y^{\prime}}, D_{z^{\prime}}\right)$, respectively. The subscript $g$ means that the operator $\mathcal{L}_{g}$ depends on the choice of the point $g \in \mathcal{K}_{1}$ (and therefore, it depends on the orbit $\mathcal{O}(g))$. Clearly, each of the operators $A_{j}\left(D_{y^{\prime}}, D_{z^{\prime}}\right)$ is properly elliptic, while the system $\left\{B_{j \rho \mu j 0}\left(D_{y^{\prime}}, D_{z^{\prime}}\right)\right\}_{\mu=1}^{m}$ satisfies the Lopatinskii condition with respect to $A_{j}\left(D_{y^{\prime}}, D_{z^{\prime}}\right)$ and is normal for all $j=1, \ldots, N$ and $\rho=1,2$.

Example 1.1. Let $Q \subset \mathbb{R}^{3}$ be a bounded domain with boundary $\partial Q \in C^{\infty}$ that is a surface of revolution about the axis $x_{3}$. Set $P=\{(0,0,3)\} \cup\{(0,0,-3)\} \cup\left\{x: x_{3}=\right.$ $\left.0, \sqrt{x_{1}^{2}+x_{2}^{2}}=3\right\}$ and $P^{1 / 4}=\{x: \operatorname{dist}(x, P)<1 / 4\}$. Assume that, outside the set $P^{1 / 4}$, the boundary $\partial Q$ coincides with the boundary of the domain

$$
\left\{x: x_{3}<3-\sqrt{x_{1}^{2}+x_{2}^{2}}\right\} \cap\left\{x: x_{3}>-3+\sqrt{x_{1}^{2}+x_{2}^{2}}\right\} \text {. }
$$

Denote

$$
\Gamma_{1}=\left\{x \in \partial Q: x_{3}<-2\right\}, \quad \Gamma_{2}=\left\{x \in \partial Q: x_{3}>2\right\}, \quad \Gamma_{3}=\partial Q \backslash\left(\overline{\Gamma_{1}} \cup \overline{\Gamma_{2}}\right) .
$$

We consider the following nonlocal boundary-value problem:

$$
\begin{aligned}
-\Delta u=f_{0}(x), \quad x \in Q, & \\
{\left.\left[u(x)+\alpha_{j} u\left(x+h_{j}\right)+\beta_{j} u\left(\mathcal{G}_{\pi} x+h_{j}\right)\right]\right|_{\Gamma_{j}} } & =0, \quad j=1,2, \\
\left.u(x)\right|_{\Gamma_{3}}=0, &
\end{aligned}
$$

where $\alpha_{j}, \beta_{j} \in \mathbb{R}, h_{j}=(-1)^{j+1}(0,0,4), j=1,2$, and $\mathcal{G}_{\pi}$ is the operator of rotation by the angle $\pi$ about the axis $x_{3}$. Clearly, we have (see Figure 1)

$$
\mathcal{K}=\mathcal{K}_{1}=\mathcal{K}_{11} \cup \mathcal{K}_{12}, \quad \mathcal{K}_{1 \nu}=\left\{x \in \partial Q: x_{3}=(-1)^{\nu} 2\right\}, \nu=1,2 .
$$

The orbit of each point $g \in \mathcal{K}_{11}$ consists of the four points: $g_{1}=g, g_{2}=\mathcal{G}_{\pi} g_{1}$, $g_{3}=g_{1}+h_{1}$, and $g_{4}=\mathcal{G}_{\pi} g_{1}+h_{1}$. Let $\hat{V}\left(g_{j}\right)=V\left(g_{j}\right)=\left\{x:\left|x-g_{j}\right|<\varepsilon\right\}$, where $\varepsilon$ is sufficiently small, and let $\operatorname{supp} u \subset\left(\bigcup_{j} V\left(g_{j}\right)\right) \cap \bar{Q}$. For $x \in V\left(g_{j}\right)$, we introduce the new variables $x^{\prime}=\left(y_{1}^{\prime}, y_{2}^{\prime}, z^{\prime}\right)$ by the formulas

$$
y_{1}^{\prime}=r-1, \quad y_{2}^{\prime}=x_{3}-g_{j 3}, \quad z^{\prime}=\varphi-\varphi_{j},
$$

where $r, \varphi, x_{3}$ and $1, \varphi_{j}, g_{j 3}$ are the cylindrical coordinates of the points $x$ and $g_{j}$, respectively. Clearly, the transformation $x \mapsto x^{\prime}(g, j)$ is nondegenerate for $x \neq 0$, and each open set $V\left(g_{j}\right)$ is taken onto one and the same neighborhood of the origin $V(0)$ under this transformation. We define the vector-valued function $v\left(x^{\prime}\right)$ such that $v_{j}\left(x^{\prime}\right)=u_{j}\left(x\left(x^{\prime}\right)\right)$ for $x^{\prime} \in V(0)$, where $u_{j}(x)=u(x)$ for $x \in V\left(g_{j}\right) \cap Q$. Denote $x^{\prime}=\left(y_{1}^{\prime}, y_{2}^{\prime}, z^{\prime}\right)$ by $x=\left(y_{1}, y_{2}, z\right)$ again. Then the boundary-value problem (1.12), (1.13) takes the form (see Figure 2)

$$
\begin{aligned}
-\frac{\partial^{2} v_{j}}{\partial y_{1}^{2}}- & \frac{\partial^{2} v_{j}}{\partial y_{2}^{2}}-\frac{1}{\left(1+y_{1}\right)^{2}} \frac{\partial^{2} v_{j}}{\partial z^{2}}-\frac{1}{1+y_{1}} \frac{\partial v_{j}}{\partial y_{1}}=f_{j}(x), \quad x \in \Theta_{j}, j=1, \ldots, 4, \\
& \left.v_{j}\right|_{\Gamma_{j 1}}=0, \quad j=1, \ldots, 4, \\
& \left.\left(v_{1}+\alpha_{1} v_{3}+\beta_{1} v_{4}\right)\right|_{\Gamma_{12}}=\left.\left(v_{2}+\beta_{1} v_{3}+\alpha_{1} v_{4}\right)\right|_{\Gamma_{22}}=0, \\
& \left.\left(v_{3}+\alpha_{2} v_{1}+\beta_{2} v_{2}\right)\right|_{\Gamma_{32}}=\left.\left(v_{4}+\beta_{2} v_{1}+\alpha_{2} v_{2}\right)\right|_{\Gamma_{42}}=0 .
\end{aligned}
$$




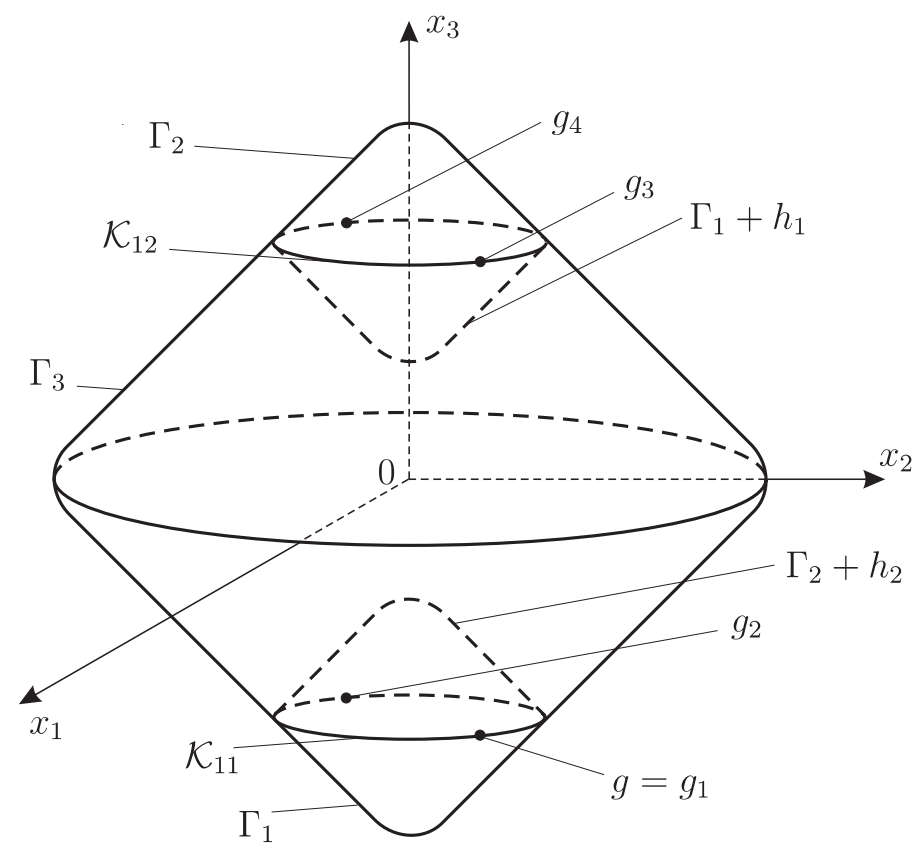

Figure 1. Problem (1.12), (1.13)

Here

$$
\begin{array}{cl}
\Theta_{1}=\Theta_{2}=\left\{x \in \mathbb{R}^{3}: y_{2}>y_{1}\right\}, & \Theta_{3}=\Theta_{4}=\left\{x \in \mathbb{R}^{3}: y_{2}<-y_{1}\right\}, \\
\Gamma_{11}=\Gamma_{21}=\left\{x \in \mathbb{R}^{3}: y_{2}=y_{1}, y_{1}>0\right\}, & \Gamma_{31}=\Gamma_{41}=\left\{x \in \mathbb{R}^{3}: y_{2}=-y_{1}, y_{1}>0\right\}, \\
\Gamma_{12}=\Gamma_{22}=\left\{x \in \mathbb{R}^{3}: y_{2}=y_{1}, y_{1}<0\right\}, & \Gamma_{32}=\Gamma_{42}=\left\{x \in \mathbb{R}^{3}: y_{2}=-y_{1}, y_{1}<0\right\} .
\end{array}
$$
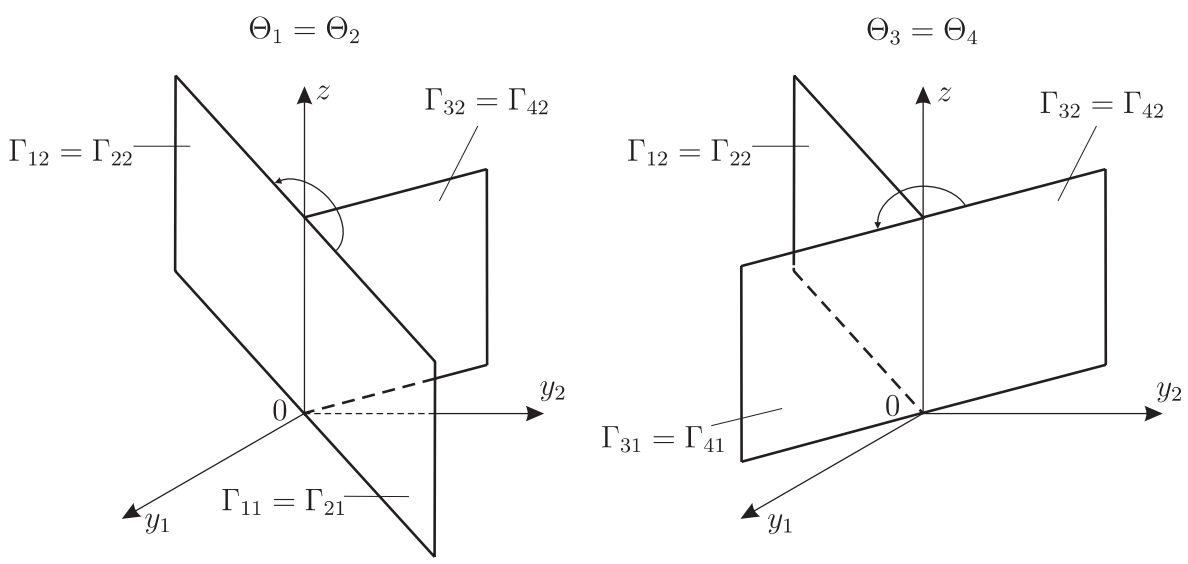

Figure 2. Problem (1.14), (1.15)

Clearly, Conditions 1.1 1.4 hold in this example. 
Passing to the principal homogeneous parts in (1.14) and freezing the coefficients at the origin, we obtain

$$
-\Delta v_{j}=f_{j}(x), \quad x \in \Theta_{j}, j=1, \ldots, 4 .
$$

The nonlocal boundary conditions (1.15) do not change.

1.4. Now fix an arbitrary point $g \in \mathcal{K}_{2}$. Clearly, $g \in \mathcal{K}_{2 \nu} \cap \Gamma_{i}$ for some $1 \leq \nu \leq$ $N_{2}$ and $1 \leq i \leq N_{0}$. By virtue of the smoothness of $\Gamma_{i}$ and $\mathcal{K}_{2 \nu}$, there exists a $C^{\infty}$ diffeomorphism $x \rightarrow x^{\prime}=x^{\prime}(g)$ defined on a small neighborhood $V(g)$ of the point $g$, such that the images of $Q \cap V(g)$ and $\mathcal{K}_{2 \nu} \cap V(g)$ are the intersection of the half-space $\mathbb{R}_{+}^{n}=\left\{x:|\varphi|<\pi / 2, z \in \mathbb{R}^{n-2}\right\}$ with some neighborhood $V(0)$ and the intersection of the set $\mathcal{P}$ with $V(0)$, respectively.

Let $A\left(D_{y^{\prime}}, D_{z^{\prime}}\right)$ and $B_{i \mu 0}\left(D_{y^{\prime}}, D_{z^{\prime}}\right)$ be the principal homogeneous parts of the operators $A\left(g, D_{y}, D_{z}\right)$ and $B_{i \mu 0}\left(g, D_{y}, D_{z}\right)$, respectively, written in the new coordinates $x^{\prime}=x^{\prime}(g)$.

We introduce the linear bounded operator

$$
\begin{aligned}
\mathcal{L}_{g}: H_{a}^{l+2 m}\left(\mathbb{R}_{+}^{n}\right) \rightarrow & \mathcal{H}_{a}^{l}\left(\mathbb{R}_{+}^{n}, \Gamma\right) \\
& =H_{a}^{l}\left(\mathbb{R}_{+}^{n}\right) \times H_{a}^{l+2 m-m_{i \mu}-1 / 2}\left(\mathbb{R}_{-}^{n-1}\right) \times H_{a}^{l+2 m-m_{i \mu}-1 / 2}\left(\mathbb{R}_{+}^{n-1}\right)
\end{aligned}
$$

given by

$$
\begin{aligned}
\mathcal{L}_{g} u=( & A\left(D_{y^{\prime}}, D_{z^{\prime}}\right) u\left(y^{\prime}, z^{\prime}\right), \\
& \left.\left.B_{i \mu 0}\left(D_{y^{\prime}}, D_{z^{\prime}}\right) u\left(y^{\prime}, z^{\prime}\right)\right|_{\varphi=-\pi / 2},\left.B_{i \mu 0}\left(D_{y^{\prime}}, D_{z^{\prime}}\right) u\left(y^{\prime}, z^{\prime}\right)\right|_{\varphi=\pi / 2}\right),
\end{aligned}
$$

where $\mathbb{R}_{ \pm}^{n-1}=\left\{x^{\prime}=\left(y^{\prime}, z^{\prime}\right) \in \mathbb{R}^{n}: \varphi= \pm \pi / 2, z^{\prime} \in \mathbb{R}^{n-2}\right\}$.

\section{Nonlocal Elliptic PROBlems IN DihedRAL ANGLES}

2.1. When studying nonlocal problems in bounded domains, we will represent the nonlocal operators as the sum of three operators. The first operator will correspond to nonlocal terms supported near the set $\mathcal{K}_{1}$, the second operator to nonlocal terms supported outside the set $\mathcal{K}_{1}$, and the third one to lower-order terms (compact perturbations). In this section, we consider a model operator corresponding to the problem with nonlocal terms supported near the set $\mathcal{K}_{1}$.

By using the Fourier transform with respect to $z$, one can reduce the study of the operator $\mathcal{L}_{g}$ in dihedral angles to the study of a model operator $\mathcal{L}_{g}(\omega)$ in plane angles, where $\omega$ is a parameter belonging to the unit sphere

$$
S^{n-3}=\left\{\omega \in \mathbb{R}^{2}:|\omega|=1\right\} ;
$$

see $[25,5$. In this section, we formulate some results (mostly proved in 25,5$]$ ) which we need below and illustrate them by an example. Note that the Fourier transform approach was earlier proposed for the study of local elliptic problems in dihedral angles [20].

To introduce the operator $\mathcal{L}_{g}(\omega)$, we preliminarily consider weighted spaces with nonhomogeneous weight. Denote by $E_{a}^{k}(\Omega)$ the completion of the set $C_{0}^{\infty}(\bar{\Omega} \backslash\{0\})$ with respect to the norm

$$
\|u\|_{E_{a}^{k}(\Omega)}=\left(\sum_{|\alpha| \leq k} \int_{\Omega} r^{2 a}\left(r^{2(|\alpha|-k)}+1\right)\left|D_{y}^{\alpha} u(y)\right|^{2} d y\right)^{1 / 2},
$$

where either $\Omega=\theta=\left\{y \in \mathbb{R}^{2}: d_{1}<\varphi<d_{2}\right\}$ or $\Omega=\mathbb{R}^{2} ; r, \varphi$ are the polar coordinates of the point $y ; k \geq 0$ is an integer. Let $\gamma \subset \bar{\Omega}$ be a half-line given by $\gamma=\left\{y \in \mathbb{R}^{2}: \varphi=\varphi_{0}\right\}$, 
where $d_{1} \leq \varphi_{0} \leq d_{2}$ for $\Omega=\theta$. Denote by $E_{a}^{k-1 / 2}(\gamma)(k \geq 1$ is an integer $)$ the space of traces on $\gamma$ with the norm

$$
\|\psi\|_{E_{a}^{k-1 / 2}(\gamma)}=\inf \|v\|_{E_{a}^{k}(\Omega)} \quad\left(v \in E_{a}^{k}(\Omega):\left.v\right|_{\gamma}=\psi\right) .
$$

Introduce the following spaces of vector-valued functions:

$$
\mathcal{E}_{a}^{k}(\theta)=\prod_{j=1}^{N} E_{a}^{k}\left(\theta_{j}\right), \quad \mathcal{E}_{a}^{l}(\theta, \gamma)=\mathcal{E}_{a}^{l}(\theta) \times \prod_{j=1}^{N} \prod_{\rho=1,2} \prod_{\mu=1}^{m} E_{a}^{l+2 m-m_{j \rho \mu}-1 / 2}\left(\gamma_{j \rho}\right),
$$

where $\theta_{j}=\left\{y \in \mathbb{R}^{2}: d_{j 1}<\varphi<d_{j 2}\right\}$ and $\gamma_{j \rho}=\left\{y \in \mathbb{R}^{2}: \varphi=d_{j \rho}\right\}$.

For a fixed point $g \in \mathcal{K}_{1}$, we consider the linear bounded operator

$$
\mathcal{L}_{g}(\omega): \mathcal{E}_{a}^{l+2 m}(\theta) \rightarrow \mathcal{E}_{a}^{l}(\theta, \gamma)
$$

given by

$$
\mathcal{L}_{g}(\omega) V=\left\{A_{j}\left(D_{y}, \omega\right) V_{j}(y),\left.\sum_{k, s}\left(B_{j \rho \mu k s}\left(D_{y}, \omega\right) V_{k}\right)\left(\mathcal{G}_{j \rho k s} y\right)\right|_{\gamma_{j \rho}}\right\}
$$

where $\omega \in S^{n-3}$ and $V=\left(V_{1}, \ldots, V_{N}\right)$; cf. (1.11).

2.2. We write the operators $A_{j}\left(D_{y}, 0\right)$ and $B_{j \rho \mu k s}\left(D_{y}, 0\right)$ in polar coordinates:

$$
A_{j}\left(D_{y}, 0\right)=r^{-2 m} \hat{A}_{j}\left(\varphi, D_{\varphi}, r D_{r}\right), \quad B_{j \rho \mu k s}\left(D_{y}, 0\right)=r^{-m_{j \rho \mu}} \hat{B}_{j \rho \mu k s}\left(\varphi, D_{\varphi}, r D_{r}\right),
$$

where $D_{\varphi}=-i \partial / \partial \varphi, D_{r}=-i \partial / \partial r$.

Introduce the following spaces of vector-valued functions:

$$
\mathcal{W}^{k}\left(d_{1}, d_{2}\right)=\prod_{j=1}^{N} W^{k}\left(d_{j 1}, d_{j 2}\right), \quad \mathcal{W}^{l}\left[d_{1}, d_{2}\right]=\mathcal{W}^{l}\left(d_{1}, d_{2}\right) \times \mathbb{C}^{m N} \times \mathbb{C}^{m N}
$$

Consider the analytic operator-valued function $\hat{\mathcal{L}}_{g}(\lambda): \mathcal{W}^{l+2 m}\left(d_{1}, d_{2}\right) \rightarrow \mathcal{W}^{l}\left[d_{1}, d_{2}\right]$ given by

$$
\begin{aligned}
\hat{\mathcal{L}}_{g}(\lambda) w= & \left\{\hat{A}_{j}\left(\varphi, D_{\varphi}, \lambda\right) w_{j},\right. \\
& \left.\left.\sum_{k, s} e^{\left(i \lambda-m_{j \rho \mu}\right) \ln \chi_{j \rho k s}}\left(\hat{B}_{j \rho \mu k s}\left(\varphi, D_{\varphi}, \lambda\right) w_{k}\right)\left(\varphi+\varphi_{j \rho k s}\right)\right|_{\varphi=d_{j \rho}}\right\},
\end{aligned}
$$

where $w=\left(w_{1}, \ldots, w_{N}\right)$.

By Lemmas 2.1 and 2.2 in 25], there exists a finite-meromorphic operator-valued function $\hat{\mathcal{R}}_{g}(\lambda): \mathcal{W}^{l}\left[d_{1}, d_{2}\right] \rightarrow \mathcal{W}^{l+2 m}\left(d_{1}, d_{2}\right)$ such that $\hat{\mathcal{L}}_{g}^{-1}(\lambda)=\hat{\mathcal{R}}_{g}(\lambda)$ for any $\lambda$ which is not a pole of $\hat{\mathcal{R}}_{g}(\lambda)$. Moreover, if $\lambda_{0}=\mu_{0}+i \nu_{0}$ is a pole of $\hat{\mathcal{R}}_{g}(\lambda)$, then $\lambda_{0}$ is an eigenvalue of $\hat{\mathcal{L}}_{g}(\lambda)$, and there exists a number $\delta>0$ such that the set $\{\lambda \in \mathbb{C}: 0<$ $\left.\left|\operatorname{Im} \lambda-\nu_{0}\right|<\delta\right\}$ contains no eigenvalues of $\hat{\mathcal{L}}_{g}(\lambda)$.

2.3. We give the following definition.

Definition 2.1. Let $H_{1}$ and $H_{2}$ denote Hilbert spaces. A linear bounded operator $L: H_{1} \rightarrow H_{2}$ is said to have the Fredholm property if $\operatorname{dim} \mathcal{N}(L)<\infty, \operatorname{codim} \mathcal{R}(L)<\infty$, and $\mathcal{R}(L)$ is closed, where $\mathcal{N}(L)$ and $\mathcal{R}(L)$ are the kernel and the image of the operator $L$, respectively.

The following theorem shows that spectral properties of the operator-valued function $\hat{\mathcal{L}}_{g}(\lambda)$ affect whether or not the operator $\mathcal{L}_{g}(\omega)$ has the Fredholm property. 
Theorem 2.1. Let Conditions 1.11 .4 hold. If the line $\operatorname{Im} \lambda=a+1-l-2 m$ contains no eigenvalues of the operator-valued function $\hat{\mathcal{L}}_{g}(\lambda)$, then the operator $\mathcal{L}_{g}(\omega): \mathcal{E}_{a}^{l+2 m}(\theta) \rightarrow$ $\mathcal{E}_{a}^{l}(\theta, \gamma)$ has the Fredholm property for all $\omega \in S^{n-3}$.

If the operator $\mathcal{L}_{g}(\omega)$ has the Fredholm property for a certain $\omega \in S^{n-3}$, then the line $\operatorname{Im} \lambda=a+1-l-2 m$ contains no eigenvalues of $\hat{\mathcal{L}}_{g}(\lambda)$.

Theorem 2.1 was proved in [5. This result is a generalization of Theorem 3.2 in [25], where one additionally assumes that the line $\operatorname{Im} \lambda=a+1-l-2 m$ contains no eigenvalues of the corresponding localized operator with a parameter $\lambda$.

The following theorem results from Theorems 3.3, 9.2, and 9.3 in [5].

Theorem 2.2. Let Conditions 1.11 .4 hold. Then the operator $\mathcal{L}_{g}: \mathcal{H}_{a}^{l+2 m}(\Theta) \rightarrow$ $\mathcal{H}_{a}^{l}(\Theta, \Gamma)$ is an isomorphism iff the operator $\mathcal{L}_{g}(\omega): \mathcal{E}_{a}^{l+2 m}(\theta) \rightarrow \mathcal{E}_{a}^{l}(\theta, \gamma)$ is an isomorphism for each $\omega \in S^{n-3}$.

Denote

$$
\mathcal{L}_{g}^{\prime} v=\left\{A_{j}^{0}\left(x, D_{y}, D_{z}\right) v_{j}(y, z),\left.\sum_{k, s}\left(B_{j \rho \mu k s}^{0}\left(x, D_{y}, D_{z}\right) v_{k}\right)\left(\mathcal{G}_{j \rho k s} y, z\right)\right|_{\Gamma_{j \rho}}\right\},
$$

where $A_{j}^{0}\left(x, D_{y}, D_{z}\right)$ and $B_{j \rho \mu k s}^{0}\left(x, D_{y}, D_{z}\right)$ are principal homogeneous parts of the operators $A_{j}\left(x, D_{y}, D_{z}\right)$ and $B_{j \rho \mu k s}\left(x, D_{y}, D_{z}\right)$, respectively. Note that $A_{j}^{0}\left(0, D_{y}, D_{z}\right)=$ $A_{j}\left(D_{y}, D_{z}\right)$ and $B_{j \rho \mu k s}^{0}\left(0, D_{y}, D_{z}\right)=B_{j \rho \mu k s}\left(D_{y}, D_{z}\right)$.

Let

$$
B_{\varepsilon}=\left\{x \in \mathbb{R}^{n}:|x|<\varepsilon\right\}, \quad \varepsilon>0,
$$

be a ball of radius $\varepsilon$ centered at the origin.

For each $\delta>0$, we define a function $\eta=\eta_{\delta} \in C_{0}^{\infty}\left(\mathbb{R}^{n}\right)$ such that $\eta(x)=1$ for $x \in B_{\delta}$, $\operatorname{supp} \eta \subset B_{2 \delta}$, and

$$
\left|D^{\beta} \eta(x)\right| \leq k_{1} \delta^{-|\beta|}, \quad x \in \mathbb{R}^{n},
$$

where $k_{1}=k_{1}(\beta)>0$ does not depend on $\delta$.

The number $\delta$ will be specified in Secs. 4 and 5 where we prove a priori estimates and construct a right regularizer for the nonlocal problem in a bounded domain.

Introduce the linear bounded operator $\mathcal{L}_{g}^{\prime \prime}: \mathcal{H}_{a}^{l+2 m}(\Theta) \rightarrow \mathcal{H}_{a}^{l}(\Theta, \Gamma)$ by the formula

$$
\mathcal{L}_{g}^{\prime \prime} v=\mathcal{L}_{g} v+\eta\left(\mathcal{L}_{g}^{\prime}-\mathcal{L}_{g}\right) v .
$$

Corollary 2.1. Let Conditions 1.11 .4 hold. Assume that the line $\operatorname{Im} \lambda=a+1-l-2 m$ contains no eigenvalues of $\hat{\mathcal{L}}_{g}(\lambda)$ and $\operatorname{dim} \mathcal{N}\left(\mathcal{L}_{g}(\omega)\right)=\operatorname{codim} \mathcal{R}\left(\mathcal{L}_{g}(\omega)\right)=0$ for any $\omega \in S^{n-3}$. Then the operator $\mathcal{L}_{g}^{\prime \prime}: \mathcal{H}_{a}^{l+2 m}(\Theta) \rightarrow \mathcal{H}_{a}^{l}(\Theta, \Gamma)$ is an isomorphism for all sufficiently small $\delta>0$ and $\left\|\left(\mathcal{L}_{g}^{\prime \prime}\right)^{-1}\right\| \leq c_{0}$, where $c_{0}>0$ does not depend on $\delta$.

Proof. Let us show that

$$
\left\|\eta\left(\mathcal{L}_{g}^{\prime}-\mathcal{L}_{g}\right)\right\| \rightarrow 0 \quad \text { as } \delta \rightarrow 0 .
$$

To do so, we first prove that

$$
\left\|\eta_{1}\left(B_{j \rho \mu k s}^{0}\left(x, D_{y}, D_{z}\right) u-B_{j \rho \mu k s}\left(D_{y}, D_{z}\right) u\right)\right\|_{H_{a}^{l+2 m-m_{j \rho \mu}\left(\Theta_{k}\right)}} \leq k_{2} \delta\|u\|_{H_{a}^{l+2 m}\left(\Theta_{k}\right)}
$$

for all $u \in H_{a}^{l+2 m}\left(\Theta_{k}\right)$, where $\eta_{1}(x)=\eta\left(\mathcal{G}_{j \rho k s}^{-1} y, z\right)$, while $k_{2}>0$ does not depend on $u$ and $\delta$.

Let

$$
b \eta_{1} D^{\beta} u, \quad|\beta|=m_{j \rho \mu},
$$

be an arbitrary term of the expression

$$
\eta_{1}\left(B_{j \rho \mu k s}^{0}\left(x, D_{y}, D_{z}\right) u-B_{j \rho \mu k s}\left(D_{y}, D_{z}\right) u\right),
$$


where $b \in C^{\infty}\left(\mathbb{R}^{n}\right)$ and $b(0)=0$. It follows from (2.4) and from the relation $b(0)=0$ that

$$
\left|r^{|\alpha|} D^{\alpha}\left(b \eta_{1}\right)\right| \leq k_{3} \delta, \quad x \in \mathbb{R}^{n},|\alpha| \leq l+2 m-m_{j \rho \mu},
$$

where $k_{3}=k_{3}(\alpha)>0$ does not depend on $\delta$. Using (2.7) and the definition of the weighted spaces, we directly derive (2.6).

Analogous relations for the pairs of the operators $A_{j}^{0}\left(x, D_{y}, D_{z}\right)$ and $A_{j}\left(D_{y}, D_{z}\right)$ can be proved in the same way. Thus, we have proved (2.5).

It follows from the conditions of this corollary and from Theorem 2.1 that the operator $\mathcal{L}_{g}(\omega)$ is an isomorphism for any $\omega \in S^{n-3}$. Therefore, by Theorem 2.2, the operator $\mathcal{L}_{g}$ is an isomorphism. Combining this fact with relation (2.5), we complete the proof.

2.4. In this subsection, we give an example of an operator which corresponds to a nonlocal elliptic problem in a dihedral angle and is an isomorphism.

Example 2.1. Let

$$
\Theta=\left\{x=(y, z) \in \mathbb{R}^{3}: 0<\varphi<d, 0<r, z \in \mathbb{R}\right\}
$$

be a three-dimensional dihedral angle, where $r, \varphi$ are the polar coordinates of the point $y$. Let

$$
\begin{aligned}
& \Gamma_{1}=\left\{x=(y, z) \in \mathbb{R}^{3}: \varphi=0,0<r, z \in \mathbb{R}\right\}, \\
& \Gamma_{2}=\left\{x=(y, z) \in \mathbb{R}^{3}: \varphi=d, 0<r, z \in \mathbb{R}\right\}
\end{aligned}
$$

be the sides of the angle $\Theta$. Consider the nonlocal elliptic problem

$$
\begin{array}{cc}
-\Delta v(x)=f_{0}(x), & x \in \Theta, \\
\left.v(\varphi, r, z)\right|_{\Gamma_{1}}-\left.\alpha_{1} v(\varphi+d / 2, r, z)\right|_{\Gamma_{1}}=f_{1}(x), & x \in \Gamma_{1}, \\
\left.v(\varphi, r, z)\right|_{\Gamma_{2}}-\left.\alpha_{2} v(\varphi-d / 2, r, z)\right|_{\Gamma_{2}}=f_{2}(x), & x \in \Gamma_{2},
\end{array}
$$

where $\alpha_{1}, \alpha_{2} \in \mathbb{R}$. Thus, the values of the unknown function $v$ on the sides $\Gamma_{1}$ and $\Gamma_{2}$ are connected with the values of $v$ on the half-plane $\left\{x=(y, z) \in \mathbb{R}^{3}: \varphi=d / 2,0<r, z \in\right.$ $\mathbb{R}\}$ lying strictly inside the angle $\Theta$. The nonlocal transformations are rotations in the $y$-plane only, while transformations with respect to the variables $r$ and $z$ are absent.

Introduce the linear bounded operator

$$
\mathcal{L}: H_{a}^{2}(\Theta) \rightarrow \mathcal{H}_{a}^{0}(\Theta, \Gamma)=H_{a}^{0}(\Theta) \times H_{a}^{3 / 2}\left(\Gamma_{1}\right) \times H_{a}^{3 / 2}\left(\Gamma_{2}\right)
$$

by the formula

$$
\mathcal{L} v=\left(-\Delta v,\left.v(\varphi, r, z)\right|_{\Gamma_{1}}-\left.\alpha_{1} v(\varphi+d / 2, r, z)\right|_{\Gamma_{1}},\left.v(\varphi, r, z)\right|_{\Gamma_{2}}-\left.\alpha_{2} v(\varphi-d / 2, r, z)\right|_{\Gamma_{2}}\right) ;
$$

cf. (1.11). Along with the operator $\mathcal{L}$, we consider the linear bounded operator

$$
\mathcal{L}(\omega): E_{a}^{2}(\theta) \rightarrow \mathcal{E}_{a}^{0}(\theta, \gamma)=E_{a}^{0}(\theta) \times E_{a}^{3 / 2}\left(\gamma_{1}\right) \times E_{a}^{3 / 2}\left(\gamma_{2}\right)
$$

given by

$\mathcal{L}(\omega) V=\left(-\Delta_{y} V+V,\left.V(\varphi, r)\right|_{\gamma_{1}}-\left.\alpha_{1} V(\varphi+d / 2, r)\right|_{\gamma_{1}},\left.V(\varphi, r)\right|_{\gamma_{2}}-\left.\alpha_{2} V(\varphi-d / 2, r)\right|_{\gamma_{2}}\right)$,

where

$$
\begin{aligned}
\theta & =\left\{y \in \mathbb{R}^{2}: 0<\varphi<d, 0<r\right\}, \\
\gamma_{1}=\left\{y \in \mathbb{R}^{2}: \varphi\right. & =0,0<r\}, \quad \gamma_{2}=\left\{y \in \mathbb{R}^{2}: \varphi=d, 0<r\right\},
\end{aligned}
$$

$\omega= \pm 1$; cf. (2.1). (Actually, one must write $-\Delta_{y} V+\omega^{2} V$ instead of $-\Delta_{y} V+V$ in the definition of the operator $\mathcal{L}(\omega)$, but $\omega^{2}=1$ for $\omega= \pm 1$. Thus, the operator $\mathcal{L}(\omega)$ does not depend on $\omega$ in this example.) 
The operator-valued function $\hat{\mathcal{L}}(\lambda): W^{2}(0, d) \rightarrow \mathcal{W}^{0}[0, d]=L_{2}(0, d) \times \mathbb{C}^{2}$ corresponding to the operator $\mathcal{L}(\omega)$ has the form

$$
\hat{\mathcal{L}}(\lambda) u=\left(-u_{\varphi \varphi}+\lambda^{2} u,\left.u\right|_{\varphi=0}-\left.\alpha_{1} u\right|_{\varphi=d / 2},\left.u\right|_{\varphi=d}-\left.\alpha_{2} u\right|_{\varphi=d / 2}\right) ;
$$

cf. (2.2).

We prove that the operator $\mathcal{L}(\omega)$ is an isomorphism for $0 \leq a \leq 2,0<\left|\alpha_{1}+\alpha_{2}\right|<2$, and $0<d<2 \arctan \sqrt{4\left(\alpha_{1}+\alpha_{2}\right)^{-2}-1}$. In this case, Theorem 2.2 implies that the operator $\mathcal{L}$ is also an isomorphism.

The proof comprises three parts.

(1) We prove that the equation

$$
\mathcal{A}_{\alpha} w=f_{0}
$$

has a unique solution for any $f_{0} \in L_{2}(\theta)$, where $\mathcal{A}_{\alpha}: \mathrm{D}\left(\mathcal{A}_{\alpha}\right) \subset L_{2}(\theta) \rightarrow L_{2}(\theta)$ is the linear bounded operator given by

$$
\begin{gathered}
\mathcal{A}_{\alpha} w=-\Delta w+w, \quad w \in \mathrm{D}\left(\mathcal{A}_{\alpha}\right)=\left\{w \in W_{\alpha}^{1}(\theta):-\Delta w+w \in L_{2}(\theta)\right\}, \\
W_{\alpha}^{1}(\theta)=\left\{w \in W^{1}(\theta):\left.w(\varphi, r)\right|_{\gamma_{1}}-\left.\alpha_{1} w(\varphi+d / 2, r)\right|_{\gamma_{1}}=0,\right. \\
\left.\left.w(\varphi, r)\right|_{\gamma_{2}}-\left.\alpha_{2} w(\varphi-d / 2, r)\right|_{\gamma_{2}}=0\right\} .
\end{gathered}
$$

To prove the unique solvability of (2.10), we reduce it to an elliptic functional differential equation.

(2) We show that each solution of (2.10) belongs to $H_{1}^{2}\left(\theta \cap B_{R}\right)$ for all $R>0$.

(3) We prove that the equation

$$
\mathcal{L}(\omega) V=f
$$

has a unique solution for any $f=\left(f_{0}, f_{1}, f_{2}\right) \in \mathcal{E}_{a}^{0}(\theta, \gamma)$.

1. Let us prove that (2.10) has a unique solution $w \in \mathrm{D}\left(\mathcal{A}_{\alpha}\right)$ for any $f_{0} \in L_{2}(\theta)$. To do this, we reduce (2.10) to a functional differential equation.

1a. Consider the functional operator $\mathcal{R}: L_{2}\left(\mathbb{R}^{2}\right) \rightarrow L_{2}\left(\mathbb{R}^{2}\right)$ given by

$$
\mathcal{R} u=u(\varphi, r)+\alpha_{1} u(\varphi+d / 2, r)+\alpha_{2} u(\varphi-d / 2, r) .
$$

Let $I_{\theta}: L_{2}(\theta) \rightarrow L_{2}\left(\mathbb{R}^{2}\right)$ denote the operator which extends a function defined on $\theta$ to $\mathbb{R}^{2}$ by zero and $P_{\theta}: L_{2}\left(\mathbb{R}^{2}\right) \rightarrow L_{2}(\theta)$ the operator which restricts a function defined on $\mathbb{R}^{2}$ to $\theta$. Set

$$
\mathcal{R}_{\theta}=P_{\theta} \mathcal{R} I_{\theta} .
$$

We claim that the operator $\mathcal{R}_{\theta}$ has the bounded inverse

$$
\mathcal{R}_{\theta}^{-1}=P_{\theta} \mathcal{R}^{\prime} I_{\theta},
$$

where

$$
\mathcal{R}^{\prime} u=\left(u(\varphi, r)-\alpha_{1} u(\varphi+d / 2, r)-\alpha_{2} u(\varphi-d / 2, r)\right) /\left(1-\alpha_{1} \alpha_{2}\right),
$$

provided that $\alpha_{1} \alpha_{2} \neq 1$ (which is true because $\left|\alpha_{1}+\alpha_{2}\right|<2$ ). Indeed,

$$
\begin{array}{ll}
\mathcal{R}_{\theta} u=u(\varphi, r)+\alpha_{1} u(\varphi+d / 2, r) & \text { for } y \in \theta_{1}=\left\{y \in \mathbb{R}^{2}: 0<\varphi<d / 2,0<r\right\}, \\
\mathcal{R}_{\theta} u=u(\varphi, r)+\alpha_{2} u(\varphi-d / 2, r) & \text { for } y \in \theta_{2}=\left\{y \in \mathbb{R}^{2}: d / 2<\varphi<d, 0<r\right\} .
\end{array}
$$

Therefore,

$$
\begin{aligned}
\mathcal{R}_{\theta}^{-1} \mathcal{R}_{\theta} u & =\left(u(\varphi, r)+\alpha_{1} u(\varphi+d / 2, r)-\alpha_{1} u(\varphi+d / 2, r)-\alpha_{1} \alpha_{2} u(\varphi, r)\right) /\left(1-\alpha_{1} \alpha_{2}\right) \\
& =u(\varphi, r) \quad \text { for } y \in \theta_{1}, \\
\mathcal{R}_{\theta}^{-1} \mathcal{R}_{\theta} u & =\left(u(\varphi, r)+\alpha_{2} u(\varphi-d / 2, r)-\alpha_{2} u(\varphi-d / 2, r)-\alpha_{1} \alpha_{2} u(\varphi, r)\right) /\left(1-\alpha_{1} \alpha_{2}\right) \\
& =u(\varphi, r) \quad \text { for } y \in \theta_{2},
\end{aligned}
$$


which implies $\mathcal{R}_{\theta}^{-1} \mathcal{R}_{\theta} u(y)=u(y)$ for $y \in \theta$. Similarly, we obtain $\mathcal{R}_{\theta} \mathcal{R}_{\theta}^{-1} u(y)=u(y)$ for $y \in \theta$. Moreover, by using the same arguments as in Theorem 8.1 in [28, Chap. 2, Sec. 8], one can verify that the operators

$$
\mathcal{R}_{\theta}: \stackrel{\circ}{W}^{1}(\theta) \rightarrow W_{\alpha}^{1}(\theta), \quad \mathcal{R}_{\theta}: \stackrel{\circ}{W}^{1}\left(\theta \cap B_{R}\right) \rightarrow W_{\alpha}^{1}\left(\theta \cap B_{R}\right)
$$

are isomorphisms for all $R>0$, where

$$
\begin{gathered}
\stackrel{\circ}{W}^{1}(\theta)=\left\{u \in W^{1}(\theta):\left.u\right|_{\gamma_{1}}=0,\left.u\right|_{\gamma_{2}}=0\right\}, \\
\stackrel{\circ}{W}^{1}\left(\theta \cap B_{R}\right)=\left\{u \in W^{1}\left(\theta \cap B_{R}\right):\left.u\right|_{\gamma_{1}}=0,\left.u\right|_{\gamma_{2}}=0\right\}, \\
W_{\alpha}^{1}\left(\theta \cap B_{R}\right)=\left\{w \in W^{1}\left(\theta \cap B_{R}\right):\left.w(\varphi, r)\right|_{\gamma_{1}}-\left.\alpha_{1} w(\varphi+d / 2, r)\right|_{\gamma_{1}}=0,\right. \\
\left.\left.w(\varphi, r)\right|_{\gamma_{2}}-\left.\alpha_{2} w(\varphi-d / 2, r)\right|_{\gamma_{2}}=0\right\} .
\end{gathered}
$$

1b. It follows from what has been proved in part 1a that (2.10) is equivalent to the equation

$$
\mathcal{A}_{\mathcal{R}} u=f_{0},
$$

where $\mathcal{A}_{\mathcal{R}}: \mathrm{D}\left(\mathcal{A}_{\mathcal{R}}\right) \subset L_{2}(\theta) \rightarrow L_{2}(\theta)$ is the unbounded operator given by

$$
\mathcal{A}_{\mathcal{R}} u=(-\Delta+I) \mathcal{R}_{\theta} u, \quad u \in \mathrm{D}\left(\mathcal{A}_{\mathcal{R}}\right)=\left\{u \in \stackrel{\circ}{W}^{1}(\theta):(-\Delta+I) \mathcal{R}_{\theta} u \in L_{2}(\theta)\right\},
$$

and $I$ stands for the identity operator in $L_{2}(\theta)$.

Similarly to Theorem 10.1 in [28, Chap. 2, Sec. 10], one can show that (2.13) has a unique solution for any $f_{0} \in L_{2}(\theta)$. However, for the reader's convenience, we prefer to give the proof here.

Consider the sesquilinear form $b_{\mathcal{R}}[u, v]$ with the domain $\mathrm{D}\left(b_{\mathcal{R}}\right)=\stackrel{\circ}{W}^{1}(\theta)$ given by

$$
b_{\mathcal{R}}[u, v]=\int_{\theta}\left(\sum_{i=1,2}\left(\mathcal{R}_{\theta} u\right)_{y_{i}} \overline{v_{y_{i}}}+\mathcal{R}_{\theta} u \bar{v}\right) d y .
$$

It is clear that

$$
\mathcal{R}_{\theta} u_{y_{i}}=\left(\mathcal{R}_{\theta} u\right)_{y_{i}} \quad \text { for } u \in \stackrel{\circ}{W}^{1}(\theta) .
$$

It follows from the Schwarz inequality and from (2.15) that

$$
\left|b_{\mathcal{R}}[u, v]\right| \leq k_{1}\|u\|_{\mathscr{W}^{1}(\theta)}\|v\|_{\mathscr{W}^{1}(\theta)},
$$

where $k_{1}>0$ does not depend on $u$ and $v$.

Introduce the isomorphism $\mathcal{U}: L_{2}(\theta) \rightarrow L_{2}\left(\theta_{1}\right) \times L_{2}\left(\theta_{1}\right)$ by the formula

$$
(\mathcal{U} u)_{i}(y)=u(\varphi+(i-1) d / 2, r), \quad y \in \theta_{1}, i=1,2 .
$$

Let $R_{1}=\left(\begin{array}{cc}1 & \alpha_{1} \\ \alpha_{2} & 1\end{array}\right)$. One can directly verify that

$$
R_{\theta} u=\mathcal{U}^{-1} R_{1} \mathcal{U} u=\mathcal{U}^{*} R_{1} \mathcal{U} u
$$

The symmetric part of the matrix $R_{1}$ has the form

$$
\left(R_{1}+R_{1}^{*}\right) / 2=\left(\begin{array}{cc}
1 & \left(\alpha_{1}+\alpha_{2}\right) / 2 \\
\left(\alpha_{1}+\alpha_{2}\right) / 2 & 1
\end{array}\right) .
$$


Since $\left|\alpha_{1}+\alpha_{2}\right|<2$, it follows that the matrix $\left(R_{1}+R_{1}^{*}\right) / 2$ is positive definite. Therefore, using (2.15) and (2.17), we obtain

$$
\begin{aligned}
\operatorname{Re} b_{\mathcal{R}}[u, u] & =\int_{\theta_{1}}\left\{\sum_{i}\left(\frac{\left(R_{1}+R_{1}^{*}\right)}{2}\left(\mathcal{U} u_{y_{i}}\right), \mathcal{U} u_{y_{i}}\right)_{\mathbb{C}^{2}}+\left(\frac{\left(R_{1}+R_{1}^{*}\right)}{2} \mathcal{U} u, \mathcal{U} u\right)_{\mathbb{C}^{2}}\right\} d y \\
& \geq k_{2} \int_{\theta_{1}}\left\{\sum_{i}\left(\mathcal{U} u_{y_{i}}, \mathcal{U} u_{y_{i}}\right)_{\mathbb{C}^{2}}+(\mathcal{U} u, \mathcal{U} u)_{\mathbb{C}^{2}}\right\} d y=k_{2}\|u\|_{W^{1}(\theta)}^{2},
\end{aligned}
$$

where $k_{2}>0$ does not depend on $u$.

Inequalities (2.16) and (2.18) imply that $b_{\mathcal{R}}$ is a closed sectorial form on $L_{2}(\theta)$, with the domain $\mathrm{D}\left(b_{\mathcal{R}}\right)=\stackrel{\circ}{W}^{1}(\theta)$ and vertex $k_{2}>0$ (see [13, Chap. 6]). It follows from the first representation theorem (see [13, Chap. 6, Sec. 2]) that the $m$-sectorial operator $\mathcal{A}_{\mathcal{R}}$ associated with the form $b_{\mathcal{R}}$ has a bounded inverse $\mathcal{A}_{\mathcal{R}}^{-1}: L_{2}(\theta) \rightarrow \stackrel{\circ}{W}^{1}(\theta)$.

Thus, we have proved that (2.10) has a unique solution $w=\mathcal{R}_{\theta} \mathcal{A}_{\mathcal{R}}^{-1} f_{0} \in \mathrm{D}\left(\mathcal{A}_{\alpha}\right)$ for any $f_{0} \in L_{2}(\theta)$.

2. We now prove that, if $w \in \mathrm{D}\left(\mathcal{A}_{\alpha}\right)$ is a solution of (2.10), then $w \in H_{1}^{2}\left(\theta \cap B_{R}(0)\right)$ for any $R>0$.

2a. Denote $\theta^{s j}=\theta \cap\left\{2^{s-j}<|y|<2^{s+j}\right\}, \gamma_{\rho}^{s j}=\gamma_{\rho} \cap\left\{2^{s-j}<|y|<2^{s+j}\right\}$, where $s=0, \pm 1, \pm 2, \ldots ; \rho, j=1,2,3 ; \gamma_{3}=\left\{y \in \mathbb{R}^{2}: \varphi=d / 2,0<r\right\}$.

We prove that $w \in W^{2}\left(\theta^{s 3}\right)$ for any $s$. By the theorem on interior smoothness (see, e.g., Theorem 3.2 in [19, Chap. 2, Sec. 3]), we have $\left.w\right|_{\gamma_{3}^{s 3}} \in W^{3 / 2}\left(\gamma_{3}^{s 3}\right)$. Since

$$
\left.w(\varphi, r)\right|_{\gamma_{1}}=\left.\alpha_{1} w(\varphi+d / 2, r)\right|_{\gamma_{1}},\left.\quad w(\varphi, r)\right|_{\gamma_{2}}=\left.\alpha_{2} w(\varphi-d / 2, r)\right|_{\gamma_{2}},
$$

it follows that

$$
\left.w\right|_{\gamma_{1}^{s 3}} \in W^{3 / 2}\left(\gamma_{1}^{s 3}\right),\left.\quad w\right|_{\gamma_{2}^{s 3}} \in W^{3 / 2}\left(\gamma_{2}^{s 3}\right) .
$$

Therefore, using a theorem on smoothness of solutions of local boundary-value problems in bounded domains (see, e.g., Theorem 8.2 in [19, Chap. 2, Sec. 8]), we obtain $w \in$ $W^{2}\left(\theta^{s 2}\right)$. Since $\theta^{s 3}=\theta^{s-1,2} \cup \theta^{s+1,2}$, it follows that $w \in W^{2}\left(\theta^{s 3}\right)$ for any $s$.

2b. Let us prove that

$$
\|w\|_{W^{2}\left(\theta^{01}\right)} \leq k_{3}\left(\|-\Delta w\|_{L_{2}\left(\theta^{03}\right)}+\|w\|_{W^{1}\left(\theta^{03}\right)}\right)
$$

where $k_{3}, k_{4}, \ldots>0$ do not depend on $w$.

To do this, we denote $\theta_{3}^{02}=\left\{y \in \theta^{02}: d / 4<\varphi<3 d / 4\right\}$ and consider a function $\xi_{0} \in C_{0}^{\infty}\left(\theta^{03}\right)$ such that $\xi_{0}(y)=1$ for $y \in \theta_{3}^{02}$.

Using the a priori estimate for solutions of local elliptic problems (see, e.g., Theorem 8.2 in [19, Chap. 2, Sec. 8] and Theorem 9.1 in [19, Chap. 2, Sec. 9]) and Leibniz' formula, we have

$$
\begin{aligned}
\left\|\left.w\right|_{\gamma_{3}^{02}}\right\|_{W^{3 / 2}\left(\gamma_{3}^{02}\right)} & \leq\|w\|_{W^{2}\left(\theta_{3}^{02}\right)} \leq\left\|\xi_{0} w\right\|_{W^{2}\left(\theta^{03}\right)} \\
& \leq k_{4}\left\|-\Delta\left(\xi_{0} w\right)\right\|_{L_{2}\left(\theta^{03}\right)} \leq k_{5}\left(\|-\Delta w\|_{L_{2}\left(\theta^{03}\right)}+\|w\|_{W^{1}\left(\theta^{03}\right)}\right) .
\end{aligned}
$$

Introduce a function $\xi_{1}(r) \in C_{0}^{\infty}(0,+\infty)$ such that $\xi_{1}(r)=1$ for $2^{-1} \leq r \leq 2$ and $\operatorname{supp} \xi_{1} \subset\left(2^{-2}, 2^{2}\right)$. Applying Theorem 8.2 in [19, Chap. 2, Sec. 8] and Theorem 9.1 in [19, Chap. 2, Sec. 9] again and using (2.20), we obtain

$$
\begin{aligned}
\|w\|_{W^{2}\left(\theta^{01}\right)} & \leq\left\|\xi_{1} w\right\|_{W^{2}\left(\theta^{02}\right)} \leq k_{6}\left(\left\|-\Delta\left(\xi_{1} w\right)\right\|_{L_{2}\left(\theta^{02}\right)}+\sum_{\rho=1,2}\left\|\left.\left(\xi_{1} w\right)\right|_{\gamma_{\rho}^{02}}\right\|_{W^{3 / 2}\left(\gamma_{\rho}^{02}\right)}\right) \\
& \leq k_{7}\left(\|-\Delta w\|_{L_{2}\left(\theta^{03}\right)}+\|w\|_{W^{1}\left(\theta^{03}\right)}\right) .
\end{aligned}
$$

Thus, inequality (2.19) is proved.

2c. Now we prove that $w \in H_{1}^{2}\left(\theta \cap B_{R}\right)$ for any $R>0$. It follows from part 2 a of the proof that $w \in H_{1}^{2}\left(\theta^{s j}\right)$ (we set $\rho(y)=|y|$ in the definition of the space $H_{1}^{2}\left(\theta^{s j}\right)$ ). Set 
$y^{\prime}=2^{-s} y$. Clearly, $y^{\prime} \in \theta^{0 j}$ for $y \in \theta^{s j}$. Therefore, using the fact that $2^{s-1}<r<2^{s+1}$ for $y \in \theta^{s 1}$, letting $w^{s}\left(y^{\prime}\right)=w\left(2^{s} y^{\prime}\right)$ and applying inequality (2.19), we obtain

$$
\begin{aligned}
\|w\|_{H_{1}^{2}\left(\theta^{s 1}\right)} & \leq k_{8} \sum_{|\alpha| \leq 2} 2^{2 s(1-2+|\alpha|)} \int_{\theta^{s 1}}\left|D_{y}^{\alpha} w(y)\right|^{2} d y \\
& =k_{8} \sum_{|\alpha| \leq 2} \int_{\theta^{01}}\left|D_{y^{\prime}}^{\alpha} w^{s}\left(y^{\prime}\right)\right|^{2} d y^{\prime} \\
& \leq k_{9}\left(\left\|-\Delta_{y^{\prime}} w^{s}\left(y^{\prime}\right)\right\|_{L_{2}\left(\theta^{03}\right)}^{2}+\sum_{|\alpha| \leq 1}\left\|D_{y^{\prime}}^{\alpha} w^{s}\left(y^{\prime}\right)\right\|_{L_{2}\left(\theta^{03}\right)}^{2}\right) \\
& =k_{9}\left(2^{2 s}\left\|-\Delta_{y} w(y)\right\|_{L_{2}\left(\theta^{s 3}\right)}^{2}+\sum_{|\alpha| \leq 1} 2^{2 s(0-1+|\alpha|)}\left\|D_{y}^{\alpha} w(y)\right\|_{L_{2}\left(\theta^{s 3}\right)}^{2}\right),
\end{aligned}
$$

where $k_{7}, k_{8}, \ldots>0$ do not depend on $w$ and $s$. It follows from (2.21) that

$$
\|w\|_{H_{1}^{2}\left(\theta^{s 1}\right)} \leq k_{10}\left(\|-\Delta w+w\|_{L_{2}\left(\theta^{s 3}\right)}^{2}+\|w\|_{H_{0}^{1}\left(\theta^{s 3}\right)}\right)
$$

for $s \leq\left[\log _{2} R\right]$. Now we claim that

$$
w \in H_{0}^{1}\left(\theta \cap B_{8 R}\right) .
$$

Indeed,

$$
w \in W_{\alpha}^{1}\left(\theta \cap B_{8 R}\right)
$$

by assumption. Therefore, $\mathcal{R}_{\theta}^{-1} w \in \stackrel{\circ}{W}^{1}\left(\theta \cap B_{8 R}\right)$ because the operator $\mathcal{R}_{\theta}: \stackrel{\circ}{W}^{1}(\theta \cap$ $\left.B_{8 R}\right) \rightarrow W_{\alpha}^{1}\left(\theta \cap B_{8 R}\right)$ is an isomorphism. By Lemma 4.8 in [15], $\mathscr{W}^{1}\left(\theta \cap B_{8 R}\right) \subset$ $H_{0}^{1}\left(\theta \cap B_{8 R}\right)$, which implies that $\mathcal{R}_{\theta}^{-1} w \in H_{0}^{1}\left(\theta \cap B_{8 R}\right)$. Therefore, using (2.12), we have

$$
w \in H_{0}^{1}\left(\theta_{1} \cap B_{8 R}\right), \quad w \in H_{0}^{1}\left(\theta_{2} \cap B_{8 R}\right) .
$$

Combining these relations with (2.24) yields (2.23).

Summing inequalities (2.22) with respect to $s \leq\left[\log _{2} R\right]$ and taking into account relation (2.23), we obtain

$$
\|w\|_{H_{1}^{2}\left(\theta \cap B_{R}\right)} \leq k_{11}\left(\|-\Delta w+w\|_{L_{2}\left(\theta \cap B_{8 R}\right)}^{2}+\|w\|_{H_{0}^{1}\left(\theta \cap B_{8 R}\right)}\right) .
$$

Thus, we have proved that $w \in H_{1}^{2}\left(\theta \cap B_{R}\right)$.

3. We finally prove that (2.11) has a unique solution $V \in E_{a}^{2}(\theta)$ for any $f \in \mathcal{E}_{a}^{0}(\theta, \gamma)$, where $0 \leq a \leq 2$.

3a. Let $w \in \mathrm{D}\left(\mathcal{A}_{\alpha}\right)$ be a solution of (2.10) with right-hand side $f_{0} \in C_{0}^{\infty}(\bar{\theta} \backslash\{0\})$. It is easy to check that the strip $-1 \leq \operatorname{Im} \lambda \leq 1$ contains no eigenvalues of the operatorvalued function $\hat{\mathcal{L}}(\lambda)$ for $0<d<2 \arctan \sqrt{4\left(\alpha_{1}+\alpha_{2}\right)^{-2}-1}$ (see [9, Sec. 9.1]). On the other hand, $w \in H_{1}^{2}\left(\theta \cap B_{1}\right)$ by the above, and the inequalities $-1 \leq a+1-2 \leq 1$ hold. Therefore, by Lemma 3.2 in 24] concerning the asymptotic behavior of solutions of nonlocal elliptic problems in plane angles, we have $w \in H_{a}^{2}\left(\theta \cap B_{1}\right)$.

$3 \mathrm{~b}$. Now let us prove that the equation

$$
\mathcal{L}(\omega) w=\left(F_{0}, 0,0\right)
$$

has a solution $w \in E_{a}^{2}(\theta)$ for any $F_{0} \in E_{a}^{0}(\theta)$.

Repeating the arguments from the proof of inequality (2.4) in [22, Chap. 6, Sec. 2], one can see that a solution $w \in \mathrm{D}\left(\mathcal{A}_{\alpha}\right)$ of (2.10) with right-hand side $f_{0} \in C_{0}^{\infty}(\bar{\theta} \backslash\{0\})$ belongs to $E_{a}^{2}\left(\theta \backslash B_{1 / 2}\right)$. Combining this fact with part 3a of our proof yields $w \in E_{a}^{2}(\theta)$. Since the line $\operatorname{Im} \lambda=a+1-2$ contains no eigenvalues of the operator-valued function $\hat{\mathcal{L}}(\lambda)$, it follows from Theorem 2.1 that the set of functions $F_{0} \in E_{a}^{0}(\theta)$ for which (2.25) 
has a solution is closed in $E_{a}^{0}(\theta)$. On the other hand, the set $C_{0}^{\infty}(\bar{\theta} \backslash\{0\})$ is dense in $E_{a}^{0}(\theta)$. Therefore, (2.25) has a solution $w \in E_{a}^{2}(\theta)$ for any $F_{0} \in E_{a}^{0}(\theta)$.

3c. Let us show that $\mathcal{R}(\mathcal{L}(\omega))=\mathcal{E}_{a}^{0}(\theta, \gamma)$. Take functions $U_{\rho} \in E_{a}^{2}(\theta)$ such that $\left.U_{\rho}\right|_{\gamma_{\rho}}=f_{\rho}, \rho=1,2$. Consider cut-off functions $\eta_{\rho}(\varphi) \in C^{\infty}[0, d]$ such that $\eta_{1}(\varphi)=1$ for $0 \leq \varphi \leq d / 4, \eta_{1}(\varphi)=0$ for $d / 3 \leq \varphi \leq d$ and $\eta_{2}(\varphi)=1$ for $3 d / 4 \leq \varphi \leq d, \eta_{2}(\varphi)=0$ for $0 \leq \varphi \leq 2 d / 3$. Then (2.11) is equivalent to (2.25), where $F_{0}=\Delta U-U+f_{0}$, $U=\eta_{1} U_{1}+\eta_{2} U_{2} \in E_{a}^{2}(\theta)$, and $w=V-U$. It is proved in part $3 \mathrm{~b}$ that (2.25) has a solution $w \in E_{a}^{2}(\theta)$ for any $F_{0} \in E_{a}^{0}(\theta)$. Therefore, (2.11) has a solution $V=w+U \in E_{a}^{2}(\theta)$ for any $f \in \mathcal{E}_{a}^{0}(\theta, \gamma)$.

3d. It remains to prove that $\mathcal{N}(\mathcal{L}(\omega))=\{0\}$. Let $w \in E_{a}^{2}(\theta)$ be a solution of (2.25) with $F_{0}=0$. Using the same arguments as in part 3a of this proof, we have $w \in H_{1}^{2}\left(\theta \cap B_{1}\right) \subset W^{1}\left(\theta \cap B_{1}\right)$. On the other hand, $E_{a}^{2}\left(\theta \backslash \overline{B_{1 / 2}}\right) \subset W^{1}\left(\theta \backslash \overline{B_{1 / 2}}\right)$ because $a \geq 0$. Therefore, $w \in W^{1}(\theta)$, and $w=0$ by part 1 of this proof.

Note that Example 2.1 was earlier studied by another method in [5, Sec. 10]. The approach proposed in [5] is based on the Green formulas for nonlocal elliptic problems (see [5]) and on the interrelation between nonlocal elliptic problems and boundary-value problems for functional differential equations (see [28]). Other examples of nonlocal elliptic problems generalizing problem (2.8), (2.9) and being uniquely solvable in dihedral angles are constructed in [23].

2.5. Given a point $g \in \mathcal{K}_{2}$, we consider the linear bounded operator

$$
\mathcal{L}_{g}(\omega): E_{a}^{l+2 m}\left(\mathbb{R}_{+}^{2}\right) \rightarrow \mathcal{E}_{a}^{l}\left(\mathbb{R}_{+}^{2}, \gamma\right)
$$

given by

$$
\mathcal{L}_{g}(\omega) V=\left(A\left(D_{y}, \omega\right) V(y),\left.B_{i \mu 0}\left(D_{y}, \omega\right) V(y)\right|_{\mathbb{R}_{-}},\left.B_{i \mu 0}\left(D_{y}, \omega\right) V(y)\right|_{\mathbb{R}_{+}}\right),
$$

where

$$
\mathcal{E}_{a}^{l}\left(\mathbb{R}_{+}^{2}, \gamma\right)=E_{a}^{l}\left(\mathbb{R}_{+}^{2}\right) \times E_{a}^{l+2 m-m_{i \mu}-1 / 2}\left(\mathbb{R}_{-}\right) \times E_{a}^{l+2 m-m_{i \mu}-1 / 2}\left(\mathbb{R}_{+}\right),
$$

$\mathbb{R}_{+}^{2}=\left\{y \in \mathbb{R}^{2}:|\varphi|<\pi / 2\right\}, \mathbb{R}_{ \pm}=\left\{y \in \mathbb{R}^{2}: \varphi= \pm \pi / 2\right\}, \omega \in S^{n-3}$; cf. (1.16).

We write the operators $A\left(D_{y}, 0\right)$ and $B_{i \mu 0}\left(D_{y}, 0\right)$ in polar coordinates:

$$
A\left(D_{y}, 0\right)=r^{-2 m} \hat{A}\left(\varphi, D_{\varphi}, r D_{r}\right), \quad B_{i \mu 0}\left(D_{y}, 0\right)=r^{-m_{i \mu}} \hat{B}_{i \mu 0}\left(\varphi, D_{\varphi}, r D_{r}\right) .
$$

Consider the analytic operator-valued function

$$
\hat{\mathcal{L}}_{g}(\lambda): W^{l+2 m}(-\pi / 2, \pi / 2) \rightarrow \mathcal{W}^{l}[-\pi / 2, \pi / 2]
$$

given by

$$
\hat{\mathcal{L}}_{g}(\lambda) w=\left(\hat{A}\left(\varphi, D_{\varphi}, \lambda\right) w,\left.\hat{B}_{i \mu 0}\left(\varphi, D_{\varphi}, \lambda\right) w\right|_{\varphi=-\pi / 2},\left.\hat{B}_{i \mu 0}\left(\varphi, D_{\varphi}, \lambda\right) w\right|_{\varphi=\pi / 2}\right),
$$

where $\mathcal{W}^{l}[-\pi / 2, \pi / 2]=W^{l}(-\pi / 2, \pi / 2) \times \mathbb{C} \times \mathbb{C}$; cf. (2.2).

It follows from [2, 4 that there exists a finite-meromorphic operator-valued function $\hat{\mathcal{R}}_{g}(\lambda): \mathcal{W}^{l}[-\pi / 2, \pi / 2] \rightarrow \mathcal{W}^{l+2 m}(-\pi / 2, \pi / 2)$ such that $\hat{\mathcal{L}}_{g}^{-1}(\lambda)=\hat{\mathcal{R}}_{g}(\lambda)$ for any $\lambda$ that is not a pole of $\hat{\mathcal{R}}_{g}(\lambda)$. Moreover, if $\lambda_{0}=\mu_{0}+i \nu_{0}$ is a pole of $\hat{\mathcal{R}}_{g}(\lambda)$, then $\lambda_{0}$ is an eigenvalue of $\hat{\mathcal{L}}_{g}(\lambda)$, and there exists a $\delta>0$ such that the set $\left\{\lambda \in \mathbb{C}: 0<\left|\operatorname{Im} \lambda-\nu_{0}\right|<\delta\right\}$ contains no eigenvalues of $\hat{\mathcal{L}}_{g}(\lambda)$.

The following theorem establishes a connection between the operators $\mathcal{L}_{g}$ and $\mathcal{L}_{g}(\omega)$ (see Theorem 2.1 in [22, Chap. 6, Sec. 2]).

Theorem 2.3. Let Conditions 1.1 and 1.2 hold. Assume that the line $\operatorname{Im} \lambda=a+1-l-2 m$ contains no eigenvalues of $\hat{\mathcal{L}}_{g}(\lambda)$ and $\operatorname{dim} \mathcal{N}\left(\mathcal{L}_{g}(\omega)\right)=\operatorname{codim} \mathcal{R}\left(\mathcal{L}_{g}(\omega)\right)=0$ for any $\omega \in S^{n-3}$. Then the operator $\mathcal{L}_{g}: \mathcal{H}_{a}^{l+2 m}\left(\mathbb{R}_{+}^{n}\right) \rightarrow \mathcal{H}_{a}^{l}\left(\mathbb{R}_{+}^{n}, \Gamma\right)$ is an isomorphism. 


\section{LoCAL ELLiptic PROBLEMS IN $\mathbb{R}^{n} \backslash \mathcal{P}$}

3.1. In Secs. 3.1 and 3.2, we recall some known results on the solvability of elliptic problems in $\mathbb{R}^{2} \backslash\{0\}$. These results are adopted from [24, 6]; they will be applied to the investigation of local elliptic problems in $\mathbb{R}^{n} \backslash \mathcal{P}, n \geq 3$; see Secs. 3.3 3.5.

Let $A$ be a properly elliptic homogeneous operator with constant complex coefficients given by

$$
A=A\left(D_{y}\right)=\sum_{|\alpha|=2 m} a_{\alpha} D_{y}^{\alpha}
$$

The operator $A: H_{a}^{l+2 m}\left(\mathbb{R}^{2}\right) \rightarrow H_{a}^{l}\left(\mathbb{R}^{2}\right)$ is bounded for any fixed integer $l \geq 0$. We consider the equation

$$
A v=f_{0}(y), \quad y \in \mathbb{R}^{2} \backslash\{0\},
$$

where $f_{0} \in H_{a}^{l}\left(\mathbb{R}^{2}\right)$.

Write the operator $A\left(D_{y}\right)$ in polar coordinates:

$$
A\left(D_{y}\right)=r^{-2 m} \hat{A}\left(\varphi, D_{\varphi}, r D_{r}\right)=r^{-2 m} \sum_{\alpha_{1}+\alpha_{2} \leq 2 m} a_{\alpha_{1} \alpha_{2}}(\varphi) D_{\varphi}^{\alpha_{1}}\left(r D_{r}\right)^{\alpha_{2}},
$$

where $a_{\alpha_{1} \alpha_{2}} \in C_{2 \pi}^{\infty}[0,2 \pi]$, and $C_{2 \pi}^{\infty}[0,2 \pi]$ is the set of functions defined on the interval $[0,2 \pi]$ such that their $2 \pi$-periodic extensions are infinitely differentiable on $\mathbb{R}$.

Setting $\tau=\ln r$, we infer from (3.1) that

$$
\begin{gathered}
\hat{A}\left(\varphi, D_{\varphi}, D_{\tau}\right) v=F_{0}(\varphi, \tau), \quad 0<\varphi<2 \pi,-\infty<\tau<\infty \\
\left.D_{\varphi}^{j} v\right|_{\varphi=0}=\left.D_{\varphi}^{j} v\right|_{\varphi=2 \pi}, \quad-\infty<\tau<\infty, j=0, \ldots, l+2 m-1,
\end{gathered}
$$

where $D_{\tau}=-i \partial / \partial \tau, F_{0}(\varphi, \tau)=e^{2 m \tau} f_{0}(\varphi, \tau),\left.D_{\varphi}^{j} F_{0}\right|_{\varphi=0}=\left.D_{\varphi}^{j} F_{0}\right|_{\varphi=2 \pi}, j=1, \ldots, l-1$.

By using the Fourier transform with respect to $\tau$, we obtain from relations (3.2) and (3.3) that

$$
\begin{gathered}
\hat{A}\left(\varphi, D_{\varphi}, \lambda\right) \hat{v}(\varphi, \lambda)=\hat{F}_{0}(\varphi, \lambda), \quad 0<\varphi<2 \pi, \\
\left.D_{\varphi}^{j} \hat{v}\right|_{\varphi=0}=\left.D_{\varphi}^{j} \hat{v}\right|_{\varphi=2 \pi}, \quad j=0, \ldots, l+2 m-1 .
\end{gathered}
$$

Denote by $W_{2 \pi}^{k}(0,2 \pi)$ the closure of the set $C_{2 \pi}^{\infty}[0,2 \pi]$ in the space $W^{k}(0,2 \pi)$. Consider the operator-valued function $\hat{A}(\lambda): W_{2 \pi}^{l+2 m}(0,2 \pi) \rightarrow W_{2 \pi}^{l}(0,2 \pi)$ given by

$$
\hat{A}(\lambda) w=\hat{A}\left(\varphi, D_{\varphi}, \lambda\right) w(\varphi) .
$$

It follows from [24, Sec. 1] that there exists a finite-meromorphic operator-valued function $\hat{\mathcal{R}}(\lambda): \mathcal{W}_{2 \pi}^{l}(0,2 \pi) \rightarrow W_{2 \pi}^{l+2 m}(0,2 \pi)$ such that $\hat{A}^{-1}(\lambda)=\hat{\mathcal{R}}(\lambda)$ for any $\lambda$ that is not a pole of $\hat{\mathcal{R}}(\lambda)$. Moreover, if $\lambda_{0}=\mu_{0}+i \nu_{0}$ is a pole of $\hat{\mathcal{R}}(\lambda)$, then $\lambda_{0}$ is an eigenvalue of $\hat{A}(\lambda)$, and there exists a $\delta>0$ such that the set $\left\{\lambda \in \mathbb{C}: 0<\left|\operatorname{Im} \lambda-\nu_{0}\right|<\delta\right\}$ contains no eigenvalues of $\hat{A}(\lambda)$.

The following result is proved in [24, Sec. 1].

Lemma 3.1. Assume that the line $\operatorname{Im} \lambda=a+1-l-2 m$ contains no eigenvalues of the operator-valued function $\hat{A}(\lambda)$. Then (3.1) has a unique solution $v \in H_{a}^{l+2 m}\left(\mathbb{R}^{2}\right)$ for any $f_{0} \in H_{a}^{l}\left(\mathbb{R}^{2}\right)$ and

$$
\|v\|_{H_{a}^{l+2 m}\left(\mathbb{R}^{2}\right)} \leq c\left\|f_{0}\right\|_{H_{a}^{l}\left(\mathbb{R}^{2}\right)},
$$

where $c>0$ does not depend on $f_{0}$. 
3.2. Now we consider the asymptotic behavior of solutions of elliptic problems in $\mathbb{R}^{2} \backslash$ $\{0\}$. Let $l_{1}, l_{2} \geq 0$ be integers, and let $a_{1}, a_{2} \in \mathbb{R}$ be such that

$$
h_{2}=a_{2}+1-l_{2}-2 m<a_{1}+1-l_{1}-2 m=h_{1} .
$$

By the above properties of the operator-valued function $\hat{A}(\lambda)$, the strip $h_{2}<\operatorname{Im} \lambda<h_{1}$ contains finitely many eigenvalues $\lambda_{j}, j=1, \ldots, J$, of $\hat{A}(\lambda)$. Let $q_{j}$ be the geometrical multiplicity of the eigenvalue $\lambda_{j}$. Denote by

$$
\left\{\psi_{j}^{0, q}(\varphi), \ldots, \psi_{j}^{p_{j q}-1, q}(\varphi)\right\}, \quad q=1, \ldots, q_{j},
$$

a canonical system of Jordan chains corresponding to the eigenvalue $\lambda_{j}$, where $p_{j 1} \geq$ $p_{j 2} \geq \cdots \geq p_{j q_{j}}$ are the ranks of the eigenvectors $\psi_{j}^{0,1}(\varphi), \ldots, \psi_{j}^{0, q_{j}}(\varphi)$, respectively; see [4, Sec. 1]. It is known that the Jordan chain (3.7) satisfies the equations

$$
\sum_{s=0}^{p} \frac{1}{s !} \partial_{\lambda}^{s} \hat{A}\left(\lambda_{j}\right) \psi_{j}^{p-s, q}(\varphi)=0, \quad p=0, \ldots, p_{j q}-1,
$$

where $\partial_{\lambda}^{s}=\partial^{s} / \partial \lambda^{s}$.

Lemma 3.2. Let $u \in H_{a_{1}}^{l_{1}+2 m}\left(\mathbb{R}^{2}\right)$ be a solution of (3.1), and let $f_{0} \in H_{a_{1}}^{l_{1}}\left(\mathbb{R}^{2}\right) \cap H_{a_{2}}^{l_{2}}\left(\mathbb{R}^{2}\right)$. Suppose that the line $\operatorname{Im} \lambda=h_{2}$ contains no eigenvalues of the operator-valued function $\hat{A}(\lambda)$. Then

$$
v(y)=\sum_{j=1}^{J} \sum_{q=1}^{q_{j}} \sum_{k=0}^{p_{j q}-1} \alpha_{j}^{k q} v_{j}^{k q}(y)+w(y), \quad y \in \mathbb{R}^{2} \backslash\{0\}
$$

here

$$
v_{j}^{k q}(y)=r^{i \lambda_{j}} \sum_{n=0}^{k} \frac{1}{n !}(i \ln r)^{n} \psi_{j}^{k-n, q}(\varphi),
$$

$\alpha_{j}^{k q}=\alpha_{j}^{k q}\left(f_{0}\right)$ are linear bounded functionals on $H_{a_{1}}^{l_{1}}\left(\mathbb{R}^{2}\right) \cap H_{a_{2}}^{l_{2}}\left(\mathbb{R}^{2}\right)$, the function $w \in$ $H_{a_{2}}^{l_{2}+2 m}\left(\mathbb{R}^{2}\right)$ satisfies the equation $A w=f_{0}$ and the inequality

$$
\|w\|_{H_{a_{2}}^{l_{2}+2 m}\left(\mathbb{R}^{2}\right)} \leq c\left\|f_{0}\right\|_{H_{a_{2}}^{l_{2}\left(\mathbb{R}^{2}\right)}}
$$

where $c>0$ does not depend on $f_{0}$.

This lemma was obtained in [24, Sec. 3] in a slightly different form. Its proof is similar to that of Theorem 1.4 in [22, Chap. 3, Sec. 1]; see also [6, Sec. 5], where the coefficients $\alpha_{j}^{k q}$ are explicitly calculated.

Remark 3.1. It is easy to see that $\psi_{j}^{s, q} \in C_{2 \pi}^{\infty}[0,2 \pi], j=1, \ldots, J ; q=1, \ldots, q_{j} ; s=$ $0, \ldots, p_{j q}-1$.

Remark 3.2. Lemma 3.2 is also valid for $h_{2} \geq h_{1}$.

Using the same arguments as in [22, Chap. 3, Sec. 1], one can obtain the following corollaries from Lemma 3.2 (see also [6, Sec. 5]).

Corollary 3.1. Let the conditions of Lemma 3.2 hold, and let the strip $h_{2}<\operatorname{Im} \lambda<h_{1}$ contain no eigenvalues of $\hat{A}(\lambda)$. Then $v \in H_{a_{2}}^{l_{2}+2 m}\left(\mathbb{R}^{2}\right)$.

Corollary 3.2. Let the line $\operatorname{Im} \lambda=a+1-l-2 m$ contain an eigenvalue of $\hat{A}(\lambda)$. Then the kernel of the operator $A: H_{a}^{l+2 m}\left(\mathbb{R}^{2}\right) \rightarrow H_{a}^{l}\left(\mathbb{R}^{2}\right)$ is trivial, while the image of $A$ is not closed in $H_{a}^{l}\left(\mathbb{R}^{2}\right)$. 
Example 3.1. We consider the equation

$$
-\Delta v(y)=f_{0}(y), \quad y \in \mathbb{R}^{2} \backslash\{0\} .
$$

Introduce the operator $A: H_{a}^{2}\left(\mathbb{R}^{2}\right) \rightarrow H_{a}^{0}\left(\mathbb{R}^{2}\right)$ by the formula $A v=-\Delta v$.

Passing to the variables $\tau, \varphi$ and using the Fourier transform with respect to $\tau$, we have

$$
\begin{gathered}
-\hat{v}_{\varphi \varphi}(\varphi, \lambda)+\lambda^{2} \hat{v}(\varphi, \lambda)=\hat{F}_{0}(\varphi, \lambda), \quad 0<\varphi<2 \pi, \\
\left.\hat{v}\right|_{\varphi=0}=\left.\hat{v}\right|_{\varphi=2 \pi},\left.\quad \hat{v}_{\varphi}\right|_{\varphi=0}=\left.\hat{v}_{\varphi}\right|_{\varphi=2 \pi},
\end{gathered}
$$

where $F_{0}(\varphi, \tau)=e^{2 \tau} f_{0}(\varphi, \tau)$; cf. (3.4), (3.5).

Let us study the eigenvalue problem for the corresponding operator-valued function $\hat{A}(\lambda): W_{2 \pi}^{2}(0,2 \pi) \rightarrow L_{2}(0,2 \pi)$ given by

$$
\hat{A}(\lambda) \hat{v}=-\hat{v}_{\varphi \varphi}+\lambda^{2} \hat{v} .
$$

The general solution of the equation

$$
-\hat{v}_{\varphi \varphi}+\lambda^{2} \hat{v}=0
$$

for $\lambda \neq 0$ has the form

$$
\hat{v}(\varphi)=c_{1} e^{\lambda \varphi}+c_{2} e^{-\lambda \varphi} .
$$

Substituting this solution into (3.14), we have

$$
\begin{aligned}
c_{1}\left(1-e^{\lambda 2 \pi}\right)+c_{2}\left(1-e^{-\lambda 2 \pi}\right) & =0, \\
c_{1} \lambda\left(1-e^{\lambda 2 \pi}\right)-c_{2} \lambda\left(1-e^{-\lambda 2 \pi}\right) & =0 .
\end{aligned}
$$

Therefore, the set of nonzero eigenvalues of $\hat{A}(\lambda)$ coincides with the set of nonzero roots of the equation

$$
e^{\lambda 2 \pi}=1
$$

The nonzero roots of this equation have the form

$$
\lambda_{k}=i k, \quad k= \pm 1, \pm 2, \ldots .
$$

It is evident that $\lambda_{0}=0$ is also an eigenvalue of $\hat{A}(\lambda)$.

1. Let us first consider the eigenvalue $\lambda_{0}=0$. The corresponding eigenvector has the form $\psi_{0}^{0,1}(\varphi)=1$ (up to a factor). The geometric multiplicity of $\lambda_{0}=0$ is equal to 1 .

Due to (3.8), an associated vector $\psi_{0}^{1,1}(\varphi)$ must satisfy the equation

$$
\hat{A}(0) \psi_{0}^{1,1}+\partial_{\lambda} \hat{A}(0) \psi_{0}^{0,1}=0,
$$

which is equivalent to the following problem:

$$
\begin{gathered}
\left(\psi_{0}^{1,1}\right)_{\varphi \varphi}=0, \quad 0<\varphi<2 \pi \\
\left.\psi_{0}^{1,1}\right|_{\varphi=0}=\left.\psi_{0}^{1,1}\right|_{\varphi=2 \pi},\left.\quad\left(\psi_{0}^{1,1}\right)_{\varphi}\right|_{\varphi=0}=\left.\left(\psi_{0}^{1,1}\right)_{\varphi}\right|_{\varphi=2 \pi} .
\end{gathered}
$$

Hence, $\psi_{0}^{1,1}=0$ (note that an associated vector of an operator-valued function, unlike an eigenvector, can be equal to zero).

Due to (3.8), the second associated vector $\psi_{0}^{2,1}(\varphi)$ must satisfy the equation

$$
\hat{A}(0) \psi_{0}^{2,1}+\partial_{\lambda} \hat{A}(0) \psi_{0}^{1,1}+\frac{1}{2} \partial_{\lambda}^{2} \hat{A}(0) \psi_{0}^{0,1}=0,
$$

which is equivalent to the following problem:

$$
\begin{gathered}
\left(\psi_{0}^{2,1}\right)_{\varphi \varphi}=1, \quad 0<\varphi<2 \pi \\
\left.\psi_{0}^{2,1}\right|_{\varphi=0}=\left.\psi_{0}^{2,1}\right|_{\varphi=2 \pi},\left.\quad\left(\psi_{0}^{2,1}\right)_{\varphi}\right|_{\varphi=0}=\left.\left(\psi_{0}^{2,1}\right)_{\varphi}\right|_{\varphi=2 \pi} .
\end{gathered}
$$


Substituting the general solution $\psi_{0}^{2,1}(\varphi)=c_{0}+c_{1} \varphi+\varphi^{2} / 2$ of (3.18) into (3.19), we obtain

$$
2 \pi c_{1}+4 \pi^{2} / 2=0, \quad 2 \pi=0
$$

This system is incompatible, and hence there does not exist a second associated vector for $\lambda_{0}=0$.

2. Consider the eigenvalue $\lambda_{k}=i k, k= \pm 1, \pm 2, \ldots$. There are two linearly independent eigenvectors corresponding to $\lambda_{k}$,

$$
\psi_{k}^{0,1}=\sin k \varphi, \quad \psi_{k}^{0,2}=\cos k \varphi
$$

(up to a factor). Hence, the geometric multiplicity of $\lambda_{k}$ equals 2 .

Due to (3.8), an associated vector $\psi_{k}^{1, j}(\varphi)(j=1,2)$ must satisfy the equation

$$
\hat{A}(i k) \psi_{k}^{1, j}+\partial_{\lambda} \hat{A}(i k) \psi_{k}^{0, j}=0,
$$

which is equivalent to the following problem:

$$
\begin{gathered}
\left(\psi_{k}^{1, j}\right)_{\varphi \varphi}+k^{2} \psi_{k}^{1, j}=i 2 k \psi_{k}^{0, j}, \quad 0<\varphi<2 \pi \\
\left.\psi_{k}^{1, j}\right|_{\varphi=0}=\left.\psi_{k}^{1, j}\right|_{\varphi=2 \pi},\left.\quad\left(\psi_{k}^{1, j}\right)_{\varphi}\right|_{\varphi=0}=\left.\left(\psi_{k}^{1, j}\right)_{\varphi}\right|_{\varphi=2 \pi} .
\end{gathered}
$$

Substituting the general solution

$$
\begin{array}{ll}
\psi_{k}^{1,1}(\varphi)=a_{k}^{1} \cos k \varphi+b_{k}^{1} \sin k \varphi-i \varphi \cos k \varphi & \text { for } j=1, \\
\psi_{k}^{1,2}(\varphi)=a_{k}^{2} \cos k \varphi+b_{k}^{2} \sin k \varphi+i \varphi \sin k \varphi & \text { for } j=2
\end{array}
$$

of (3.20) into (3.21), we have

$$
\begin{array}{ll}
0=-i 2 \pi, \quad 0=0 & \text { for } j=1, \\
0=0, \quad 0=i 2 \pi k & \text { for } j=2 .
\end{array}
$$

These systems are incompatible for $k= \pm 1, \pm 2, \ldots$, and hence there do not exist associated vectors for $\lambda_{k}=i k, k= \pm 1, \pm 2, \ldots$.

Example 3.2. Let $v \in H_{1}^{2}\left(\mathbb{R}^{2}\right)$ be a solution of (3.12) with right-hand side $f_{0} \in H_{1}^{0}\left(\mathbb{R}^{2}\right) \cap$ $H_{-\varepsilon}^{0}\left(\mathbb{R}^{2}\right)$, where $0<\varepsilon<1$. We study the asymptotic behavior of the solution $v$. Using the results of Example 3.1 and Lemma 3.2 for $a_{1}=1, l_{1}=0, h_{1}=0$ and $a_{2}=-\varepsilon, l_{2}=$ $0, h_{2}=-1-\varepsilon$, we obtain

$(3.22) v(y)=\left(\alpha_{1}^{0,1} \sin \varphi+\alpha_{1}^{0,2} \cos \varphi\right) r+w(y)=\alpha_{1}^{0,1} y_{2}+\alpha_{1}^{0,2} y_{1}+w(y), \quad y \in \mathbb{R}^{2} \backslash\{0\}$, where $w \in H_{-\varepsilon}^{2}\left(\mathbb{R}^{2}\right)$, while $\alpha_{1}^{0, j}=\alpha_{1}^{0, j}\left(f_{0}\right)$ are linear bounded functionals on $H_{1}^{0}\left(\mathbb{R}^{2}\right) \cap$ $H_{-\varepsilon}^{0}\left(\mathbb{R}^{2}\right)$. Note that these functionals can be found in an explicit form (see [6, Sec. 5]).

3.3. We now proceed with the study of elliptic problems in $\mathbb{R}^{n} \backslash \mathcal{P}$ for $n \geq 3$, where

$$
\mathcal{P}=\left\{x=(y, z) \in \mathbb{R}^{n}: y=0, z \in \mathbb{R}^{n-2}\right\} .
$$

Let

$$
A=A\left(D_{y}, D_{z}\right)=\sum_{|\alpha|+|\beta|=2 m} a_{\alpha \beta} D_{y}^{\alpha} D_{z}^{\beta}
$$

be a homogeneous properly elliptic operator with constant complex coefficients. We consider the equation

$$
A v=f_{0}(x), \quad x \in \mathbb{R}^{n} \backslash \mathcal{P},
$$

where $f_{0} \in H_{a}^{l}\left(\mathbb{R}^{n}\right)$. It is easy to see that the operator $A: H_{a}^{l+2 m}\left(\mathbb{R}^{n}\right) \rightarrow H_{a}^{l}\left(\mathbb{R}^{n}\right)$ is bounded for any $a \in \mathbb{R}$ and any integer $l \geq 0$.

The main result of this section is as follows. 
Theorem 3.1. Suppose $a \in \mathbb{R}$, and let $l \geq 0$ be an integer. Then the operator $A$ : $H_{a}^{l+2 m}\left(\mathbb{R}^{n}\right) \rightarrow H_{a}^{l}\left(\mathbb{R}^{n}\right)$ is not an isomorphism.

To prove Theorem 3.1, we first apply the Fourier transform $F_{z \rightarrow \eta}$ with respect to $z \in \mathbb{R}^{n-2}$. Then (3.23) takes the form

$$
A\left(D_{y}, \eta\right) \tilde{v}(y, \eta)=\tilde{f}_{0}(y, \eta), \quad y \in \mathbb{R}^{2} \backslash\{0\}, \eta \in \mathbb{R}^{n-2},
$$

where

$$
\tilde{v}(y, \eta)=F_{z \rightarrow \eta} v=(2 \pi)^{-(n-2) / 2} \int_{\mathbb{R}^{n-2}} v(y, z) e^{-i(\eta, z)} d z .
$$

Denote $Y=|\eta| y, \omega=\eta /|\eta|, V(Y, \eta)=|\eta|^{2 m} \tilde{v}(y, \eta), F_{0}(Y, \eta)=\tilde{f}_{0}(y, \eta)$. Then (3.24) takes the following form:

$$
A\left(D_{Y}, \omega\right) V(Y, \eta)=F_{0}(Y, \eta), \quad y \in \mathbb{R}^{2} \backslash\{0\}, \omega \in S^{n-3} .
$$

Consider the linear bounded operator $A(\omega): E_{a}^{l+2 m}\left(\mathbb{R}^{2}\right) \rightarrow E_{a}^{l}\left(\mathbb{R}^{2}\right)$ given by

$$
A(\omega) V=A\left(D_{Y}, \omega\right) V(Y), \quad \omega \in S^{n-3} .
$$

Denote by $A(0): H_{a}^{l+2 m}\left(\mathbb{R}^{2}\right) \rightarrow H_{a}^{l}\left(\mathbb{R}^{2}\right)$ the linear bounded operator given by

$$
A(0) v=A\left(D_{y}, 0\right) v(y) .
$$

Clearly, the operator $A(0)$ is properly elliptic. Write the operator $A(0)$ in polar coordinates as

$$
A(0)=r^{-2 m} \hat{A}\left(\varphi, D_{\varphi}, r D_{r}\right) .
$$

Consider the operator-valued function $\hat{A}(\lambda): W_{2 \pi}^{l+2 m}(0,2 \pi) \rightarrow W_{2 \pi}^{l}(0,2 \pi)$ given by

$$
\hat{A}(\lambda) w=\hat{A}\left(\varphi, D_{\varphi}, \lambda\right) w(\varphi) .
$$

Spectral properties of the operator-valued function $\hat{A}(\lambda)$ are described in Sec. 3.1 .

The proof of the following lemma is similar to that of Theorem 2.3 in 22, Chap. 6, Sec. 2] (see also Theorem 4.2 in [25]).

Lemma 3.3. The operator $A(\omega): E_{a}^{l+2 m}\left(\mathbb{R}^{2}\right) \rightarrow E_{a}^{l}\left(\mathbb{R}^{2}\right)$ has the Fredholm property for each $\omega \in S^{n-3}$ iff the line $\operatorname{Im} \lambda=a+1-l-2 m$ contains no eigenvalues of the operator-valued function $\hat{A}(\lambda)$.

However, we prove below (see Lemma 3.10) that the operator $A(\omega): E_{a}^{l+2 m}\left(\mathbb{R}^{2}\right) \rightarrow$ $E_{a}^{l}\left(\mathbb{R}^{2}\right)$ is not an isomorphism for $a \in \mathbb{R}, l \geq 0$, and $\omega \in S^{n-3}$.

The following result is valid (see [25]).

Lemma 3.4. The operator $A: H_{a}^{l+2 m}\left(\mathbb{R}^{n}\right) \rightarrow H_{a}^{l}\left(\mathbb{R}^{n}\right)$ is an isomorphism iff the operator $A(\omega): E_{a}^{l+2 m}\left(\mathbb{R}^{2}\right) \rightarrow E_{a}^{l}\left(\mathbb{R}^{2}\right)$ is an isomorphism.

Combining Lemma 3.4 with the fact that $A(\omega)$ is not an isomorphism allows us to prove Theorem 3.1 Thus, it remains to show that the operator $A(\omega)$ is not an isomorphism for $a \in \mathbb{R}, l \geq 0$, and $\omega \in S^{n-3}$. To do so, we preliminarily establish a priori estimates for solutions of (3.25) and study the adjoint operators.

The proof of a priori estimates in the spaces $E_{a}^{l}\left(\mathbb{R}^{2}\right)$ is based on the well-known a priori estimate in Sobolev spaces (see, e.g., Theorem 15.3 in [1] and the comment following it).

Lemma 3.5. Let $Q_{1}, Q_{2} \subset \mathbb{R}^{n}$ be bounded domains such that $\overline{Q_{1}} \subset Q_{2}$. Assume that an operator

$$
\mathcal{A}=\sum_{|\alpha| \leq 2 m} a_{\alpha}(x) D_{x}^{\alpha}
$$


with infinitely differentiable coefficients $a_{\alpha}(x)$ is properly elliptic on $\overline{Q_{2}}$. Then the following estimate holds for all $u \in W^{l+2 m}\left(Q_{2}\right)$ :

$$
\|u\|_{W^{l+2 m}\left(Q_{1}\right)} \leq c\left(\|\mathcal{A} u\|_{W^{l}\left(Q_{2}\right)}+\|u\|_{L_{2}\left(Q_{2}\right)}\right),
$$

where $c>0$ depends on $Q_{1}, Q_{2}$, and $M$,

$$
M=\max _{|\beta| \leq l_{0}} \max _{|\alpha| \leq 2 m} \max _{x \in \overline{Q_{2}}}\left|D^{\beta} a_{\alpha}(x)\right|, \quad l_{0}=\max (l, 1),
$$

and does not depend on $u$.

Remark 3.3. Theorem 3.1 in [19, Chap. 2, Sec. 3] ensures the validity of estimate (3.27) with the term $\|u\|_{W^{l+2 m-1}\left(Q_{2}\right)}$ instead of $\|u\|_{L_{2}\left(Q_{2}\right)}$ on the right-hand side. To obtain estimate (3.27), one must additionally apply the technique close to that in [21, Chap. 5].

Denote by $W_{\text {loc }}^{k}\left(\mathbb{R}^{2} \backslash\{0\}\right)$ the space of distributions $v$ on $\mathbb{R}^{2} \backslash\{0\}$ such that $\psi v \in$ $W^{k}\left(\mathbb{R}^{2}\right)$ for all $\psi \in C_{0}^{\infty}\left(\mathbb{R}^{2} \backslash\{0\}\right)$.

Lemma 3.6. Let $v \in W_{\text {loc }}^{l+2 m}\left(\mathbb{R}^{2} \backslash\{0\}\right) \cap E_{a-l-2 m}^{0}(\{|y|<1\})$ and $A(\omega) v \in E_{a}^{l}\left(\mathbb{R}^{2}\right)$ for some $\omega \in S^{n-3}$. Then $v \in E_{a}^{l+2 m}\left(\mathbb{R}^{2}\right)$ and

$$
\|v\|_{E_{a}^{l+2 m}\left(\mathbb{R}^{2}\right)} \leq c\left(\|A(\omega) v\|_{E_{a}^{l}\left(\mathbb{R}^{2}\right)}+\|v\|_{E_{a-l-2 m}^{0}(\{|y|<R\})}\right),
$$

where $R, c>0$ do not depend on $v$ and $\omega$.

Proof. 1. Denote $Q_{1}^{s}=\left\{y \in \mathbb{R}^{2}: 2^{s}<|y|<2^{s+1}\right\}$ and $Q_{2}^{s}=\left\{y \in \mathbb{R}^{2}: 2^{s-1}<\right.$ $\left.|y|<2^{s+2}\right\}, s=0, \pm 1, \pm 2, \ldots$ Evidently, $\overline{Q_{1}^{s}} \subset Q_{2}^{s}$. It follows from the inclusion $v \in W_{\text {loc }}^{l+2 m}\left(\mathbb{R}^{2} \backslash\{0\}\right)$ that $v \in H_{a}^{l+2 m}\left(Q_{2}^{s}\right) \cap E_{a}^{l+2 m}\left(Q_{2}^{s}\right)$ for any $s$ (we set $\rho(y)=|y|$ in the definition of the spaces $H_{a}^{l+2 m}\left(Q_{2}^{s}\right)$ and $\left.E_{a}^{l+2 m}\left(Q_{2}^{s}\right)\right)$. First, we prove that

$$
\|v\|_{E_{a}^{l+2 m}\left(Q_{1}^{s}\right)}^{2} \leq k_{1}\left(\|A(\omega) v\|_{E_{a}^{l}\left(Q_{2}^{s}\right)}^{2}+\|v\|_{E_{a-l-2 m}^{0}\left(Q_{2}^{s}\right)}^{2}\right), \quad s \leq 0,
$$

where $k_{1}, k_{2}, \ldots>0$ do not depend on $v, \omega$, and $s$.

Set $y^{\prime}=2^{-s} y$. Clearly, $y^{\prime} \in Q_{j}^{0}$ for $y \in Q_{j}^{s}, j=1,2 ; s=0, \pm 1, \pm 2, \ldots$ Therefore, setting $v^{s}\left(y^{\prime}\right)=v\left(2^{s} y^{\prime}\right)$ and applying Lemma 3.5. we obtain, for $s \leq 0$,

$$
\begin{aligned}
\|v\|_{H_{a}^{l+2 m}\left(Q_{1}^{s}\right)}^{2} & \leq k_{2} \sum_{|\alpha| \leq l+2 m} 2^{2 s(a-l-2 m+|\alpha|)}\left\|D_{y}^{\alpha} v(y)\right\|_{L_{2}\left(Q_{1}^{s}\right)}^{2} \\
& =k_{2} \sum_{|\alpha| \leq l+2 m} 2^{2 s(a-l-2 m+1)}\left\|D_{y^{\prime}}^{\alpha} v^{s}\left(y^{\prime}\right)\right\|_{L_{2}\left(Q_{1}^{0}\right)}^{2} \\
& \leq k_{3} 2^{2 s(a-l-2 m+1)}\left(\sum_{|\alpha| \leq l}\left\|D_{y^{\prime}}^{\alpha} A\left(D_{y^{\prime}}, 2^{s} \omega\right) v^{s}\left(y^{\prime}\right)\right\|_{L_{2}\left(Q_{2}^{0}\right)}^{2}+\left\|v^{s}\left(y^{\prime}\right)\right\|_{L_{2}\left(Q_{2}^{0}\right)}^{2}\right) \\
& =k_{3}\left(\sum_{|\alpha| \leq l} 2^{2 s(a-l+|\alpha|)}\left\|D_{y}^{\alpha} A\left(D_{y}, \omega\right) v(y)\right\|_{L_{2}\left(Q_{2}^{s}\right)}^{2}+2^{2 s(a-l-2 m)}\|v(y)\|_{L_{2}\left(Q_{2}^{s}\right)}^{2}\right) \\
& \leq k_{4}\left(\left\|A\left(D_{y}, \omega\right) v\right\|_{H_{a}^{l}\left(Q_{2}^{s}\right)}^{2}+\|v\|_{H_{a-l-2 m}^{0}\left(Q_{2}^{s}\right)}^{2}\right) .
\end{aligned}
$$

The latter estimate is equivalent to (3.29).

2. To complete the proof, it remains to show that the estimate in (3.29) is also valid for $s>0$. To do so, we apply Lemma 3.5 for $\mathcal{A}=A\left(D_{y^{\prime}}, D_{z}\right), u\left(y^{\prime}, z\right)=\exp \left(i 2^{s}(\omega, z)\right) v^{s}\left(y^{\prime}\right)$, and

$$
Q_{j}=Q_{j}^{0} \times\left\{z \in \mathbb{R}^{n-2}:\left|z_{k}\right|<j, k=1, \ldots, n-2\right\}, \quad j=1,2
$$


Then we obtain

$$
\begin{aligned}
& \sum_{\nu=0}^{l+2 m} 2^{2 s \nu}\left\|v^{s}\left(y^{\prime}\right)\right\|_{W^{l+2 m-\nu}\left(Q_{1}^{0}\right)}^{2} \\
& \quad \leq k_{5}\left(\sum_{\nu=0}^{l} 2^{2 s \nu}\left\|A\left(D_{y^{\prime}}, 2^{s} \omega\right) v^{s}\left(y^{\prime}\right)\right\|_{W^{l-\nu}\left(Q_{2}^{0}\right)}^{2}+\left\|v^{s}\left(y^{\prime}\right)\right\|_{L_{2}\left(Q_{2}^{0}\right)}^{2}\right) .
\end{aligned}
$$

Since $s>0$, it follows that inequality (3.30) is equivalent to the following:

$$
\begin{aligned}
& 2^{2 s(l+2 m-1)}\|v(y)\|_{W^{l+2 m}\left(Q_{1}^{s}\right)}^{2} \\
& \quad \leq k_{6}\left(2^{2 s(l+2 m-1)}\left\|A\left(D_{y}, \omega\right) v(y)\right\|_{W^{l}\left(Q_{2}^{s}\right)}^{2}+2^{-2 s}\|v(y)\|_{L_{2}\left(Q_{2}^{s}\right)}^{2}\right) .
\end{aligned}
$$

Multiplying both sides of this inequality by $2^{2 s(a-l-2 m+1)}$ yields

$$
\|v\|_{E_{a}^{l+2 m}\left(Q_{1}^{s}\right)}^{2} \leq k_{1}\left(\|A(\omega) v\|_{E_{a}^{l}\left(Q_{2}^{s}\right)}^{2}+2^{2 s(a-l-2 m)}\|v\|_{L_{2}\left(Q_{2}^{s}\right)}^{2}\right), \quad s>0 .
$$

Summing (3.29) and (3.31) with respect to $s=0,-1,-2, \ldots$ and $s=1,2, \ldots$, respectively, and choosing a sufficiently large $R>0$, we obtain (3.28).

Using Lemmas 3.2 and 3.6. we can prove the following result on regularity of solutions of the equation

$$
A(\omega) v=f_{0}, \quad \omega \in S^{n-3} .
$$

Lemma 3.7. Let the closed strip bounded by the lines $\operatorname{Im} \lambda=a_{1}+1-l_{1}-2 m$ and $\operatorname{Im} \lambda=a_{2}+1-l_{2}-2 m$ contain no eigenvalues of the operator-valued function $\hat{A}(\lambda)$. Suppose that $v \in E_{a_{1}}^{l_{1}+2 m}\left(\mathbb{R}^{2}\right)$ is a solution of (3.32) for some $\omega \in S^{n-3}$, with right-hand side $f_{0} \in E_{a_{1}}^{l_{1}}\left(\mathbb{R}^{2}\right) \cap E_{a_{2}}^{l_{2}}\left(\mathbb{R}^{2}\right)$. Then $v \in E_{a_{2}}^{l_{2}+2 m}\left(\mathbb{R}^{2}\right)$.

Proof. 1. Consider a function $\eta \in C^{\infty}(\mathbb{R})$ such that $\eta(r)=0$ for $r \leq 1$ and $\eta(r)=$ 1 for $r \geq 2$. Denote by $[A(\omega), \eta]$ the commutator of $A(\omega)$ and $\eta$. It is clear that $\operatorname{supp}[A(\omega), \eta] v \subset\left\{y \in \mathbb{R}^{2}: 1 \leq|y| \leq 2\right\}$. Therefore,

$$
A(\omega)(\eta v)=\eta f_{0}+[A(\omega), \eta] v \in E_{a_{1}}^{l_{1}}\left(\mathbb{R}^{2}\right) \cap E_{a_{2}}^{l_{2}}\left(\mathbb{R}^{2}\right)
$$

because $v \in W_{\text {loc }}^{l+2 m}\left(\mathbb{R}^{2} \backslash\{0\}\right)$, where $l=\max \left(l_{1}, l_{2}\right)$, due to Theorem 3.2 in [19, Chap. 2, Sec. 3].

On the other hand, $\eta v$ vanishes near the origin, and hence $\eta v \in E_{a_{2}-l_{2}-2 m}^{0}(\{|y|<R\})$ for any $R>0$. Using this fact, relation (3.33), and Lemma 3.6, we conclude that $\eta v \in E_{a_{2}}^{l_{2}+2 m}\left(\mathbb{R}^{2}\right)$.

2. Since $\operatorname{supp} A(\omega)((1-\eta) v) \subset\left\{y \in \mathbb{R}^{2}:|y| \leq 2\right\}$, we obtain (similarly to (3.33)) that

$$
A(\omega)((1-\eta) v) \in H_{a_{1}}^{l_{1}}\left(\mathbb{R}^{2}\right) \cap H_{a_{2}}^{l_{2}}\left(\mathbb{R}^{2}\right) .
$$

Therefore, using Lemma 3.2 and Remark 3.2 , we conclude that $1-\eta) v \in H_{a_{2}}^{l_{2}+2 m}\left(\mathbb{R}^{2}\right)$, and hence $(1-\eta) v \in E_{a_{2}}^{l_{2}+2 m}\left(\mathbb{R}^{2}\right)$.

Thus, $v \in E_{a_{2}}^{l_{2}+2 m}\left(\mathbb{R}^{2}\right)$.

\footnotetext{
${ }^{1}$ Since the operator $A(\omega)$ contains lower-order terms, one must consecutively apply Lemma 3.2 finitely many times; cf. [15] 16.
} 
3.4. In this subsection, we consider adjoint operators. Introduce the operator

$$
A^{\prime}(\omega)=A^{\prime}\left(D_{y}, \omega\right)=\sum_{|\alpha|+|\beta|=2 m} \overline{a_{\alpha \beta}} \omega^{\beta} D_{y}^{\alpha} .
$$

The operator $A^{\prime}\left(D_{y}, \omega\right)$ is formally adjoint to $A\left(D_{y}, \omega\right)$ with respect to the Green formula, i.e.,

$$
\int_{\mathbb{R}^{2}} A\left(D_{y}, \omega\right) u \bar{v} d y=\int_{\mathbb{R}^{2}} u \overline{A^{\prime}\left(D_{y}, \omega\right) v} d y, \quad \omega \in \mathbb{R}^{n-2},
$$

for all $u, v \in C_{0}^{\infty}\left(\mathbb{R}^{2} \backslash\{0\}\right)$.

Consider the unbounded operators

$$
\begin{gathered}
\mathcal{A}(\omega): \mathrm{D}(\mathcal{A}(\omega)) \subset E_{b-2 m}^{0}\left(\mathbb{R}^{2}\right) \rightarrow E_{b}^{0}\left(\mathbb{R}^{2}\right), \\
\mathcal{A}(\omega) v=A\left(D_{y}, \omega\right) v, \quad v \in \mathrm{D}(\mathcal{A}(\omega))=E_{b}^{2 m}\left(\mathbb{R}^{2}\right)
\end{gathered}
$$

and

$$
\begin{gathered}
\mathcal{A}^{\prime}(\omega): \mathrm{D}\left(\mathcal{A}^{\prime}(\omega)\right) \subset E_{-b}^{0}\left(\mathbb{R}^{2}\right) \rightarrow E_{2 m-b}^{0}\left(\mathbb{R}^{2}\right), \\
\mathcal{A}^{\prime}(\omega) v=A^{\prime}\left(D_{y}, \omega\right) v, \quad v \in \mathrm{D}\left(\mathcal{A}^{\prime}(\omega)\right)=E_{2 m-b}^{2 m}\left(\mathbb{R}^{2}\right) .
\end{gathered}
$$

Lemma 3.8. The operator $\mathcal{A}^{\prime}(\omega)$ is adjoint to $\mathcal{A}(\omega)$ with respect to the inner product in $L_{2}\left(\mathbb{R}^{2}\right)$ for any $\omega \in S^{n-3}$.

Proof. Denote by $\mathcal{A}^{*}(\omega)$ the adjoint operator for $\mathcal{A}(\omega)$. Since $C_{0}^{\infty}\left(\mathbb{R}^{2} \backslash\{0\}\right)$ is dense in the spaces $E_{b}^{2 m}\left(\mathbb{R}^{2}\right)$ and $E_{2 m-b}^{2 m}\left(\mathbb{R}^{2}\right)$, it follows that identity (3.35) is valid for all $u \in E_{b}^{2 m}\left(\mathbb{R}^{2}\right)$ and $v \in E_{2 m-b}^{2 m}\left(\mathbb{R}^{2}\right)$. Therefore, $\mathcal{A}^{\prime}(\omega) \subset \mathcal{A}^{*}(\omega)$.

It remains to prove the inverse inclusion. Let $v \in \mathrm{D}\left(\mathcal{A}^{*}(\omega)\right) \subset E_{-b}^{0}\left(\mathbb{R}^{2}\right)$. Since $\mathcal{A}^{*}(\omega) v \in E_{2 m-b}^{0}\left(\mathbb{R}^{2}\right) \subset L_{2, \text { loc }}\left(\mathbb{R}^{2} \backslash\{0\}\right)$, it follows from Theorem 3.2 in [19, Chap. 2, Sec. 3] that $v \in W_{\text {loc }}^{2 m}\left(\mathbb{R}^{2} \backslash\{0\}\right)$. Therefore, by Lemma 3.6, $v \in E_{2 m-b}^{2 m}\left(\mathbb{R}^{2}\right)$, and hence $\mathcal{A}^{*}(\omega) \subset \mathcal{A}^{\prime}(\omega)$.

Consider the identity (3.35) for $\omega=0$, substitute $u=u_{1}$ and $v=r^{2 m-2} v_{1}$ into it, and set $\tau=\ln r$. Then we have

$$
\int_{-\infty}^{\infty} d \tau \int_{0}^{2 \pi}\left(\hat{A}\left(\varphi, D_{\varphi}, D_{\tau}\right) u_{1} \overline{v_{1}}-u_{1} \overline{\hat{A}^{\prime}\left(\varphi, D_{\varphi}, D_{\tau}-2 i(m-1)\right) v_{1}}\right) d \varphi=0
$$

for all $u_{1}, v_{1} \in\left\{u \in C_{0}^{\infty}([0,2 \pi] \times \mathbb{R}):\left.D_{\varphi}^{j} u\right|_{\varphi=0}=\left.D_{\varphi}^{j} u\right|_{\varphi=2 \pi}, j=0,1, \ldots\right\}$, where $\hat{A}^{\prime}\left(\varphi, D_{\varphi}, D_{\tau}\right)$ is defined similarly to $\hat{A}\left(\varphi, D_{\varphi}, D_{\tau}\right)$.

Consider functions $\psi, \hat{\psi} \in C_{0}^{\infty}(\mathbb{R})$ such that

$$
\begin{gathered}
\psi(\tau)=0 \quad \text { for }|\tau|>1, \quad \int_{-\infty}^{\infty} \psi(\tau) d \tau=1, \\
\hat{\psi}(\tau)=1 \quad \text { for }|\tau|<1, \quad \hat{\psi}(\tau)=0 \quad \text { for }|\tau|>2 .
\end{gathered}
$$

Substituting $u_{1}=e^{i \lambda \tau} \psi(\tau) u_{2}(\varphi)$ and $v_{1}=e^{i \bar{\lambda} \tau} \hat{\psi}(\tau) v_{2}(\varphi)$ into (3.36), we obtain

$$
\int_{0}^{2 \pi}\left(\hat{A}\left(\varphi, D_{\varphi}, \lambda\right) u_{2} \overline{v_{2}}-u_{2} \overline{\hat{A}^{\prime}\left(\varphi, D_{\varphi}, \bar{\lambda}-2 i(m-1)\right) v_{2}}\right) d \varphi=0
$$

for all $u_{2}, v_{2} \in C_{2 \pi}^{\infty}[0,2 \pi]$ and $\lambda \in \mathbb{C}$.

Along with $\hat{A}(\lambda)$, we consider the operator-valued function $\hat{A}^{\prime}(\lambda): W_{2 \pi}^{2 m}(0,2 \pi) \rightarrow$ $L_{2}(0,2 \pi)$ given by

$$
\hat{A}^{\prime}(\lambda) w=\hat{A}^{\prime}\left(\varphi, D_{\varphi}, \lambda\right) w
$$

We also introduce the unbounded operators

$$
\hat{\mathcal{A}}(\lambda), \hat{\mathcal{A}}^{\prime}(\lambda): \mathrm{D}(\hat{\mathcal{A}}(\lambda))=\mathrm{D}\left(\hat{\mathcal{A}}^{\prime}(\lambda)\right) \subset L_{2}(0,2 \pi) \rightarrow L_{2}(0,2 \pi)
$$


given by

$$
\begin{gathered}
\hat{\mathcal{A}}(\lambda) w=\hat{A}\left(\varphi, D_{\varphi}, \lambda\right) w, \quad w \in \mathrm{D}(\hat{\mathcal{A}}(\lambda))=W_{2 \pi}^{2 m}(0,2 \pi), \\
\hat{\mathcal{A}}^{\prime}(\lambda) w=\hat{A}^{\prime}\left(\varphi, D_{\varphi}, \lambda\right) w, \quad w \in \mathrm{D}\left(\hat{\mathcal{A}}^{\prime}(\lambda)\right) .
\end{gathered}
$$

Similarly to Lemma 3.8, we conclude from (3.37) that the operator $\hat{\mathcal{A}}^{\prime}(\bar{\lambda}-2 i(m-1))$ is adjoint to $\hat{\mathcal{A}}(\lambda)$ with respect to the inner product in $L_{2}(0,2 \pi)$ for any $\lambda \in \mathbb{C}$. This fact and the fact that $\hat{A}(\lambda)$ is a Fredholm operator with ind $\hat{A}(\lambda)=0$ imply:

Lemma 3.9. A number $\lambda$ is an eigenvalue of $\hat{A}(\lambda)$ iff $\bar{\lambda}-2 i(m-1)$ is an eigenvalue of $\hat{A}^{\prime}(\lambda)$.

3.5. In this subsection, using the results of Secs. 3.1 3.4 we prove the following result.

Lemma 3.10. Let $a \in \mathbb{R}, l \geq 0$ be an integer, and $\omega \in S^{n-3}$. Then the operator $A(\omega): E_{a}^{l+2 m}\left(\mathbb{R}^{2}\right) \rightarrow E_{a}^{l}\left(\mathbb{R}^{2}\right)$ is not an isomorphism.

We preliminarily prove two lemmas on the conditions for the operator $A(\omega)$ to be an isomorphism. These lemmas, together with properties of the adjoint operator, will enable us to prove Lemma 3.10 and hence Theorem 3.1 .

Lemma 3.11. Assume that the strip $a_{2}+1-l-2 m<\operatorname{Im} \lambda<a_{1}+1-l-2 m$ contains no eigenvalues of the operator-valued function $\hat{A}(\lambda)$. If the operator $A(\omega), \omega \in S^{n-3}$, is an isomorphism for some $a=a_{0} \in\left(a_{2}, a_{1}\right)$, then it is an isomorphism for all $a \in\left(a_{2}, a_{1}\right)$.

Proof. 1. It suffices to prove that $\mathcal{N}(A(\omega))=\{0\}$ and $\mathcal{R}(A(\omega))=E_{b}^{l}\left(\mathbb{R}^{2}\right)$ for each $a=b \in\left(a_{2}, a_{1}\right)$. It follows from Lemma 3.7 that, if $v \in \mathcal{N}(A(\omega))$ for $a=b, a_{2}<b<a_{1}$, then $v \in \mathcal{N}(A(\omega))$ for $a=a_{0}$. Hence $v=0$, i.e., $\mathcal{N}(A(\omega))=\{0\}$ for $a=b$.

2. Consider (3.32) for $f_{0} \in C_{0}^{\infty}\left(\mathbb{R}^{2} \backslash\{0\}\right)$ and $a=a_{0}$. By assumption, this equation has a unique solution $v \in E_{a_{0}}^{l+2 m}\left(\mathbb{R}^{2}\right)$. By virtue of Lemma 3.7, $v \in E_{b}^{l+2 m}\left(\mathbb{R}^{2}\right)$. Lemma 3.3 implies that $\mathcal{R}(A(\omega))$ (for $a=b$ ) is closed in $E_{b}^{l}\left(\mathbb{R}^{2}\right)$. Combining this with the fact that $C_{0}^{\infty}\left(\mathbb{R}^{2} \backslash\{0\}\right)$ is dense in $E_{b}^{l}\left(\mathbb{R}^{2}\right)$ yields $\mathcal{R}(A(\omega))=E_{b}^{l}\left(\mathbb{R}^{2}\right)$.

Lemma 3.12. Assume that each of the lines $\operatorname{Im} \lambda=a_{2}+1-l-2 m$ and $\operatorname{Im} \lambda=$ $a_{1}+1-l-2 m$ contains an eigenvalue of the operator-valued function $\hat{A}(\lambda)$, and let the strip $a_{2}+1-l-2 m<\operatorname{Im} \lambda<a_{1}+1-l-2 m$ contain no eigenvalues of the operatorvalued function $\hat{A}(\lambda)$. If the operator $A(\omega), \omega \in S^{n-3}$, is an isomorphism for some $a=a_{0} \in\left(a_{2}, a_{1}\right)$, then it is not an isomorphism for $a \notin\left(a_{2}, a_{1}\right)$.

Proof. 1. Let us prove that $\operatorname{dim} \mathcal{N}(A(\omega))>0$ for $a>a_{1}$. Set

$$
u=r^{i \lambda_{0}} \psi^{0}(\varphi),
$$

where $\lambda_{0}$ is an eigenvalue of the operator-valued function $\hat{A}(\lambda)$ such that $\operatorname{Im} \lambda_{0}=a_{1}+$ $1-l-2 m$ and $\psi^{0}(\varphi)$ is the corresponding eigenvector. In this case,

$$
A(0) u=0 .
$$

Therefore,

$$
A(\omega)((1-\eta) u)=[A(0),(1-\eta)] u+(A(\omega)-A(0))((1-\eta) u) \equiv F,
$$

where $\eta$ is the same function as in the proof of Lemma 3.7 .

Note that

$$
\begin{array}{ll}
(1-\eta) u \notin E_{b}^{l+2 m}\left(\mathbb{R}^{2}\right) & \text { for any } b \leq a_{1}, \\
(1-\eta) u \in E_{a}^{l+2 m}\left(\mathbb{R}^{2}\right) & \text { for any } a>a_{1}
\end{array}
$$


Clearly,

$$
F \in E_{b}^{l}\left(\mathbb{R}^{2}\right) \quad \text { for any } b \in\left(a_{1}-1,+\infty\right) .
$$

By Lemma 3.11, (3.32) with the right-hand side $f_{0}=F$ has a unique solution $v \in$ $E_{b}^{l+2 m}\left(\mathbb{R}^{2}\right)$, where $b \in\left(a_{2}, a_{1}\right) \cap\left(a_{1}-1,+\infty\right)$. In particular, this implies that $w=$ $(1-\eta) u-v$ is not the zero function due to (3.39).

Further, using the relation $v \in E_{b}^{l+2 m}\left(\mathbb{R}^{2}\right) \subset E_{b}^{0}\left(\mathbb{R}^{2}\right)$ and taking (3.41) into account, we deduce from Lemma 3.6 that $v \in E_{b+l+2 m}^{l+2 m}\left(\mathbb{R}^{2}\right)$. Repeating these arguments finitely many times, we obtain that $v \in E_{a}^{l+2 m}\left(\mathbb{R}^{2}\right)$ for any $a>a_{1}$. Combining this relation with (3.40), we see that $w \in E_{a}^{l+2 m}\left(\mathbb{R}^{2}\right)$ for $a>a_{1}$, and hence $w \in \mathcal{N}(A(\omega))$ for $a>a_{1}$.

2. Now let $a<a_{2}$. If the line $\operatorname{Im} \lambda=a+1-l-2 m$ contains an eigenvalue of $\hat{A}(\lambda)$, then the conclusion of this lemma follows from Lemma 3.3 . Therefore, we assume that the line $\operatorname{Im} \lambda=a+1-l-2 m$ contains no eigenvalues of $\hat{A}(\lambda)$. In this case, the set $\mathcal{R}(A(\omega))$ is closed both for $A(\omega): E_{a-l}^{2 m}\left(\mathbb{R}^{2}\right) \rightarrow E_{a-l}^{0}\left(\mathbb{R}^{2}\right)$ and for $A(\omega): E_{a}^{l+2 m}\left(\mathbb{R}^{2}\right) \rightarrow E_{a}^{l}\left(\mathbb{R}^{2}\right)$.

2a. First, we prove that $d=\operatorname{dim} \mathcal{R}(A(\omega))^{\perp}>0$ for

$$
A(\omega): E_{a-l}^{2 m}\left(\mathbb{R}^{2}\right) \rightarrow E_{a-l}^{0}\left(\mathbb{R}^{2}\right) .
$$

By virtue of Lemma 3.9, the lines $\operatorname{Im} \lambda=l+2 m-a_{2}+1-2 m$ and $\operatorname{Im} \lambda=l+2 m-$ $a_{1}+1-2 m$ contain eigenvalues of $\hat{A}^{\prime}(\lambda)$, while the strip $l+2 m-a_{1}+1-2 m<\operatorname{Im} \lambda<$ $l+2 m-a_{2}+1-2 m$ contains no eigenvalues of $\hat{A}^{\prime}(\lambda)$. By assumption, the operator $A(\omega): E_{a_{0}}^{l+2 m}\left(\mathbb{R}^{2}\right) \rightarrow E_{a_{0}}^{l}\left(\mathbb{R}^{2}\right), \omega \in S^{n-3}$, is an isomorphism. Therefore, by Lemmas 3.6 and 3.3. the operator $A(\omega): E_{a_{0}-l}^{2 m}\left(\mathbb{R}^{2}\right) \rightarrow E_{a_{0}-l}^{0}\left(\mathbb{R}^{2}\right)$ is also an isomorphism. Now it follows from Lemma 3.8 that the operator

$$
A^{\prime}(\omega): E_{l+2 m-a_{0}}^{2 m}\left(\mathbb{R}^{2}\right) \rightarrow E_{l+2 m-a_{0}}^{0}\left(\mathbb{R}^{2}\right)
$$

is an isomorphism. Applying part 1 of this proof to the operator $A^{\prime}(\omega)$, we conclude that $\operatorname{dim} \mathcal{N}\left(A^{\prime}(\omega)\right)>0$ for $A^{\prime}(\omega): E_{b}^{2 m}\left(\mathbb{R}^{2}\right) \rightarrow E_{b}^{0}\left(\mathbb{R}^{2}\right)$, where $b>l+2 m-a_{2}$. Therefore, by virtue of Lemma 3.8, $d>0$ for $A(\omega): E_{a-l}^{2 m}\left(\mathbb{R}^{2}\right) \rightarrow E_{a-l}^{0}\left(\mathbb{R}^{2}\right)$, where $2 m-(a-l)>$ $l+2 m-a_{2}$, i.e., $a<a_{2}$.

2b. It remains to prove that $\operatorname{dim} \mathcal{R}(A(\omega))^{\perp}=d$ for

$$
A(\omega): E_{a}^{l+2 m}\left(\mathbb{R}^{2}\right) \rightarrow E_{a}^{l}\left(\mathbb{R}^{2}\right) .
$$

Due to part $1 \mathrm{~b}$ of the proof, (3.32) with right-hand side $f_{0} \in E_{a-l}^{0}\left(\mathbb{R}^{2}\right)$ has a solution $v \in E_{a-l}^{2 m}\left(\mathbb{R}^{2}\right)$ iff

$$
\left(f_{0}, f_{j}\right)_{E_{a-l}^{0}\left(\mathbb{R}^{2}\right)}=0, \quad j=1, \ldots, d,
$$

where $f_{1}, \ldots, f_{d} \in E_{a-l}^{0}\left(\mathbb{R}^{2}\right)$ are linearly independent functions. It follows from Lemma 3.6 that conditions (3.42) are necessary and sufficient for (3.32) with right-hand side $f_{0} \in E_{a}^{l}\left(\mathbb{R}^{2}\right)$ to have a solution $v \in E_{a}^{l+2 m}\left(\mathbb{R}^{2}\right)$. It follows from the Schwarz inequality and from the boundedness of the embedding $E_{a}^{l}\left(\mathbb{R}^{2}\right) \subset E_{a-l}^{0}\left(\mathbb{R}^{2}\right)$ that

$$
\left|\left(f_{0}, f_{j}\right)_{E_{a-l}^{0}\left(\mathbb{R}^{2}\right)}\right| \leq\left\|f_{0}\right\|_{E_{a-l}^{0}\left(\mathbb{R}^{2}\right)}\left\|f_{j}\right\|_{E_{a-l}^{0}\left(\mathbb{R}^{2}\right)} \leq c\left\|f_{0}\right\|_{E_{a}^{l}\left(\mathbb{R}^{2}\right)}\left\|f_{j}\right\|_{E_{a-l}^{0}\left(\mathbb{R}^{2}\right)},
$$

where $c>0$ does not depend on $f_{0}$. Hence, by Riesz' theorem, there are functions $F_{j} \in E_{a}^{l}\left(\mathbb{R}^{2}\right), j=1, \ldots, d$, such that

$$
\left(f_{0}, f_{j}\right)_{E_{a-l}^{0}\left(\mathbb{R}^{2}\right)}=\left(f_{0}, F_{j}\right)_{E_{a}^{l}\left(\mathbb{R}^{2}\right)} \quad \text { for all } f_{0} \in E_{a}^{l}\left(\mathbb{R}^{2}\right),
$$

and the functions $F_{j}$ are linearly independent. Thus, $\operatorname{dim} \mathcal{R}(A(\omega))^{\perp}$ in $E_{a}^{l}\left(\mathbb{R}^{2}\right)$ is equal to $d$. 
Proof of Lemma 3.10, 1. First, we show that the operator

$$
A(\omega): E_{l+m}^{l+2 m}\left(\mathbb{R}^{2}\right) \rightarrow E_{l+m}^{l}\left(\mathbb{R}^{2}\right)
$$

is not an isomorphism for any $l \geq 0$. To do so, we prove that $\lambda_{0}=i(1-m)$ is an eigenvalue of $\hat{A}(\lambda)$. Consider a homogeneous polynomial $q(y)$ of order $m-1$ and write it in polar coordinates as $q(y)=r^{m-1} \tilde{q}(\varphi)$, where $\tilde{q} \in C_{2 \pi}^{\infty}[0,2 \pi]$. We have

$$
0=A\left(D_{y}, 0\right) q(y)=r^{-2 m} \hat{A}\left(\varphi, D_{\varphi}, r D_{r}\right)\left(r^{m-1} \tilde{q}(\varphi)\right)=r^{-m-1} \hat{A}\left(\varphi, D_{\varphi}, i(1-m)\right) \tilde{q}(\varphi) .
$$

Hence, $\lambda_{0}=i(1-m)$ is an eigenvalue and $\tilde{q}(\varphi)$ is the corresponding eigenvector. Since the line $\operatorname{Im} \lambda=1-m=m+1-2 m$ contains the eigenvalue $\lambda_{0}$, it follows from Lemma 3.3 that the operator $A(\omega): E_{l+m}^{l+2 m}\left(\mathbb{R}^{2}\right) \rightarrow E_{l+m}^{l}\left(\mathbb{R}^{2}\right)$ does not have the Fredholm property. Therefore, it is not an isomorphism.

2. Now we prove that the operator $A(\omega): E_{a}^{2 m}\left(\mathbb{R}^{2}\right) \rightarrow E_{a}^{0}\left(\mathbb{R}^{2}\right)$ is not an isomorphism for any $a, a \neq m$. Assume, to the contrary, that $A(\omega)$ is an isomorphism for some $a \neq m$. Then, by Lemma 3.8.

$$
\text { the operator } \quad A^{\prime}(\omega): E_{2 m-a}^{2 m}\left(\mathbb{R}^{2}\right) \rightarrow E_{2 m-a}^{0}\left(\mathbb{R}^{2}\right) \quad \text { is an isomorphism. }
$$

Note that

$$
\left.\overline{A\left(D_{y}, \omega\right) u(y)} \equiv\left[A^{\prime}\left(D_{y^{\prime}}, \omega\right) w\left(y^{\prime}\right)\right]\right|_{y^{\prime}=-y},
$$

where $w\left(y^{\prime}\right)=\overline{u\left(-y^{\prime}\right)}$. It follows from this relation and from (3.43) that the operator $A(\omega): E_{2 m-a}^{2 m}\left(\mathbb{R}^{2}\right) \rightarrow E_{2 m-a}^{0}\left(\mathbb{R}^{2}\right)$ is also an isomorphism. This contradicts Lemma 3.12 because the strip bounded by the $\operatorname{lines} \operatorname{Im} \lambda=a+1-2 m$ and $\operatorname{Im} \lambda=(2 m-a)+1-2 m$ contains the eigenvalue $\lambda_{0}=i(1-m)$ of the operator-valued function $\hat{A}(\lambda)$.

3 . Finally, we prove that the operator $A(\omega): E_{a}^{l+2 m}\left(\mathbb{R}^{2}\right) \rightarrow E_{a}^{l}\left(\mathbb{R}^{2}\right)$ is not an isomorphism for all $\omega \in S^{n-3}, l>0$, and $a \neq l+m$. Assume, to the contrary, that it is an isomorphism for some $\omega \in S^{n-3}, l>0$, and $a \neq l+m$. Then, by Lemma 3.3, the line $\operatorname{Im} \lambda=a+1-l-2 m$ contains no eigenvalues of the operator-valued function $\hat{A}(\lambda)$. Therefore, according to part 2 of this proof, either $\operatorname{dim} \mathcal{N}(A(\omega))>0$ or $\operatorname{dim} \mathcal{R}(A(\omega))^{\perp}>0$ for the operator $A(\omega): E_{a-l}^{2 m}\left(\mathbb{R}^{2}\right) \rightarrow E_{a-l}^{0}\left(\mathbb{R}^{2}\right)$.

Let $\operatorname{dim} \mathcal{N}(A(\omega))>0$ for the above operator $A(\omega)$. Hence, there exists a function $v \in E_{a-l}^{2 m}\left(\mathbb{R}^{2}\right)$ such that $v \neq 0$ and $A(\omega) v=0$. By Lemma 3.6 $v \in E_{a}^{l+2 m}\left(\mathbb{R}^{2}\right)$, and hence $\operatorname{dim} \mathcal{N}(A(\omega))>0$ for $A(\omega): E_{a}^{l+2 m}\left(\mathbb{R}^{2}\right) \rightarrow E_{a}^{l}\left(\mathbb{R}^{2}\right)$. This contradicts our assumption.

Let $\operatorname{dim} \mathcal{R}(A(\omega))^{\perp}>0$ for $A(\omega): E_{a-l}^{2 m}\left(\mathbb{R}^{2}\right) \rightarrow E_{a-l}^{0}\left(\mathbb{R}^{2}\right)$. Since $A(\omega): E_{a}^{l+2 m}\left(\mathbb{R}^{2}\right) \rightarrow$ $E_{a}^{l}\left(\mathbb{R}^{2}\right)$ is an isomorphism, it follows that the equation

$$
A(\omega) v=f_{0}
$$

has a solution $v \in E_{a}^{l+2 m}\left(\mathbb{R}^{2}\right) \subset E_{a-l}^{2 m}\left(\mathbb{R}^{2}\right)$ for each $f_{0} \in E_{a}^{l}\left(\mathbb{R}^{2}\right)$. On the other hand, $E_{a}^{l}\left(\mathbb{R}^{2}\right)$ is dense in $E_{a-l}^{0}\left(\mathbb{R}^{2}\right)$, while $\mathcal{R}(A(\omega))$ is closed in $E_{a-l}^{0}\left(\mathbb{R}^{2}\right)$ for $A(\omega): E_{a-l}^{2 m}\left(\mathbb{R}^{2}\right) \rightarrow$ $E_{a-l}^{0}\left(\mathbb{R}^{2}\right)$. Therefore, $\mathcal{R}(A(\omega))=E_{a-l}^{0}\left(\mathbb{R}^{2}\right)$, which contradicts the assumption that $\operatorname{dim} \mathcal{R}(A(\omega))^{\perp}>0$.

Theorem 3.1 follows from Lemmas 3.4 and 3.10 .

\section{A PRIORI ESTIMATES OF SOlUtions IN BOUNDED DOMAINS}

4.1. In this section, we obtain a priori estimates for solutions of nonlocal elliptic problems in weighted spaces. Combining these estimates with the existence of a right regularizer, which we construct in the next section, we prove the Fredholm property for the corresponding nonlocal operator. Let us discuss the choice of weighted spaces. For each set $\mathcal{K}_{j}, j=1,2,3$, we may assume that either the set $K$ in the definition of the spaces $H_{a}^{k}(Q)=H_{a}^{k}(Q, K)$ and $H_{a}^{k-1 / 2}(\Gamma)=H_{a}^{k-1 / 2}(\Gamma, K)$ contains the set $\mathcal{K}_{j}$ or it does not. 
This is equivalent to whether or not the right-hand sides and solutions of nonlocal problems in bounded domains have singularities near the set $\mathcal{K}_{j}$. If $\mathcal{K}_{j} \subset K$, then the model operators corresponding to the points of the set $\mathcal{K}_{j}$ and playing a fundamental role in obtaining a priori estimates and constructing a right regularizer must be considered in weighted spaces (otherwise, in Sobolev spaces).

Consider the set $\mathcal{K}_{1}=\partial Q \backslash \bigcup_{i} \Gamma_{i}$ of conjugation points. It is shown in [24, 29] (see also [10]) that generalized solutions of nonlocal problems can have power-law singularities near the set $\mathcal{K}_{1}$. Therefore, we always assume that $\mathcal{K}_{1} \subset K$, while the corresponding model operators act on weighted spaces in dihedral angles.

Consider the set $\mathcal{K}_{3} \subset Q$. It follows from Theorem 3.1 that the model operator on weighted spaces in $\mathbb{R}^{n}$ is not an isomorphism (moreover, one can show that it does not even have the Fredholm property on weighted spaces; cf. Remark 2.2 in [22, Chap. 6, Sec. 2]). Therefore, we assume that the right-hand sides and solutions of nonlocal problems in bounded domains have no singularities inside the domain $Q$, while the corresponding model operator acts on Sobolev spaces. If $\mathcal{K}_{3}=\varnothing$, this assumption leads to no difficulties. However, if $\mathcal{K}_{3} \neq \varnothing$, the following difficulty arises. Take a point $g \in \overline{\Gamma_{i}} \cap \mathcal{K}$ such that $\omega_{i s}(g) \in \mathcal{K}_{3}$, and let a function $u$ belong to the weighted space $H_{a}^{l+2 m}$ near the point $g$ and to the Sobolev space $W^{l+2 m}$ near the point $\omega_{i s}(g)$. Since $\omega_{i s}$ is a smooth nondegenerate transformation, it follows that the function $\left.\left(B_{i \mu s}(x, D) u\right)\left(\omega_{i s}(x)\right)\right|_{\Gamma_{i}}$ occurring in nonlocal conditions (1.4) belongs to $W^{l+2 m-m_{i \mu}-1 / 2}$ near the point $g$; however, in general, it does not belong to $H_{a}^{l+2 m-m_{i \mu}-1 / 2}$ near the point $g$. Therefore, the corresponding nonlocal operator appears to be unbounded on weighted spaces. To eliminate this obstacle, we additionally assume that $a>l+2 m-1$ in the case $\mathcal{K}_{3} \neq \varnothing$, which ensures the inclusion $W^{l+2 m-m_{i \mu}-1 / 2} \subset H_{a}^{l+2 m-m_{i \mu}-1 / 2}$ in a neighborhood of $g \in \overline{\Gamma_{i}} \cap \mathcal{K}$ (cf. Lemma 4.5 below).

Consider the set $\mathcal{K}_{2} \subset \bigcup_{i} \Gamma_{i}$. We may either include or not include the set $\mathcal{K}_{2}$ in the set $K$. In the first case, we consider model local operators on weighted spaces. In the second case, we consider model local operators on Sobolev spaces. The advantage of the "weighted case" is that we solve a nonlocal problem in the whole scale of spaces (depending on the weight parameter $a \in \mathbb{R}$ ). However, the disadvantage is that we must impose some assumptions on the location of the eigenvalues of an auxiliary problem with the parameter $\lambda$ and require that an auxiliary operator with the parameter $\omega \in S^{n-3}$ be an isomorphism (the latter is often hard to verify); see Theorem 2.3. The advantage of the case of Sobolev spaces is that the model operators are isomorphisms without any additional assumptions. The disadvantage is that, if $\mathcal{K}_{2} \neq \varnothing$, we must suppose $a>l+2 m-1$ even if $\mathcal{K}_{3}=\varnothing$ (the reason is similar to that in the above case $\mathcal{K}_{3} \neq \varnothing$ ).

The following consistency condition integrates all the above cases.

Condition 4.1 (Consistency condition).

(1) If $\mathcal{K}_{3}=\varnothing$, then either

(a) $a \in \mathbb{R}$ and $K=\mathcal{K}_{1} \cup \mathcal{K}_{2}$, or

(b) $a>l+2 m-1$ and $K=\mathcal{K}_{1}$.

(2) If $\mathcal{K}_{3} \neq \varnothing$, then $a>l+2 m-1$ and either

(a) $K=\mathcal{K}_{1} \cup \mathcal{K}_{2}$, or

(b) $K=\mathcal{K}_{1}$.

To conclude this subsection, we prove two auxiliary results. Denote

$$
\mathcal{M}^{\delta}=\left\{x \in \mathbb{R}^{n}: \operatorname{dist}(x, \mathcal{M})<\delta\right\}
$$

for any set $\mathcal{M} \subset \mathbb{R}^{n}$ and $\delta>0$. 
Lemma 4.1. Let $\zeta \in C^{\infty}\left(\mathbb{R}^{n}\right)$ be a function such that $\zeta(x)=0$ for $x \in \mathcal{K}_{1}$. Then

$$
\|\zeta v\|_{H_{a}^{l}(Q)} \leq c \delta\|v\|_{H_{a}^{l}(Q)}
$$

for all $v \in H_{a}^{l}(Q)$ such that $\operatorname{supp} v \subset \bar{Q} \cap \mathcal{K}_{1}^{\delta}$, where $c>0$ does not depend on $\delta$ and $v$.

Proof. Since $\zeta(x)=0$ for $x \in \mathcal{K}_{1}$, it follows from the Taylor formula that

$$
|\zeta(x)| \leq k_{1} \delta, \quad x \in \mathcal{K}_{1}^{\delta} .
$$

Therefore, using (4.2), we obtain

$$
\begin{aligned}
\|\zeta v\|_{H_{a}^{l}(Q)}^{2}= & \sum_{|\beta| \leq l} \int_{Q \cap \mathcal{K}_{1}^{\delta}} \rho^{2(a-l+|\beta|)}\left|\zeta D^{\beta} v\right|^{2} d x \\
& +\sum_{|\alpha|=1}^{l} \sum_{|\beta| \leq l-|\alpha|} \int_{Q \cap \mathcal{K}_{1}^{\delta}} \rho^{2|\alpha|} \rho^{2(a-l+|\beta|)}\left|D^{\alpha} \zeta D^{\beta} v\right|^{2} d x \\
\leq & \sum_{|\beta| \leq l} k_{1}^{2} \delta^{2} \int_{Q \cap \mathcal{K}_{1}^{\delta}} \rho^{2(a-l+|\beta|)}\left|D^{\beta} v\right|^{2} d x \\
& +\sum_{|\alpha|=1}^{l} \sum_{|\beta| \leq l-|\alpha|} k_{2} \delta^{2|\alpha|} \int_{Q \cap \mathcal{K}_{1}^{\delta}} \rho^{2(a-l+|\beta|)}\left|D^{\beta} v\right|^{2} d x,
\end{aligned}
$$

which implies (4.1).

Lemma 4.2. Let $\zeta_{\delta} \in C^{\infty}\left(\mathbb{R}^{n}\right)$ be a family of functions such that $\operatorname{supp} \zeta_{\delta} \subset \mathcal{K}_{1}^{\delta}$ and

$$
\left|D^{\beta} \zeta_{\delta}(x)\right| \leq c_{1} \delta^{-|\beta|}, \quad x \in Q, \quad|\beta| \leq l,
$$

where $c_{1}>0$ does not depend on $\delta$. Then

$$
\left\|\zeta_{\delta} u\right\|_{H_{a}^{l}(Q)} \leq c_{2}\|u\|_{H_{a}^{l}(Q)}
$$

for all $u \in H_{a}^{l}(Q)$, where $c_{2}>0$ does not depend on $\delta$ and $u$.

Proof. Using (4.3), we obtain

$$
\begin{aligned}
\left\|\zeta_{\delta} u\right\|_{H_{a}^{l}(Q)}^{2} & =\sum_{|\alpha|+|\beta| \leq l} \int_{Q \cap \mathcal{K}_{1}^{\delta}} \rho^{2|\alpha|} \rho^{2(a-l+|\beta|)}\left|D^{\alpha} \zeta_{\delta} D^{\beta} u\right|^{2} d x \\
& \leq k \sum_{|\beta| \leq l} \int_{Q \cap \mathcal{K}_{1}^{\delta}} \rho^{2(a-l+|\beta|)}\left|D^{\beta} u\right|^{2} d x
\end{aligned}
$$

which implies (4.4).

Remark 4.1. Lemmas 4.1 and 4.2 are true for the spaces $H_{a}^{l}(Q)$ replaced by $H_{a}^{l-1 / 2}(\Gamma)$, where $\Gamma$ is a smooth $(n-1)$-dimensional manifold such that $\bar{\Gamma} \subset \bar{Q}$ and $l-1 / 2 \geq 1 / 2$. To prove this, it suffices to use the corresponding bounded operator of extension acting from $H_{a}^{l-1 / 2}(\Gamma)$ to $H_{a}^{l}(Q)$.

4.2. We introduce the linear operator

$$
\mathbf{L}=\left\{A, B_{i \mu}\right\}
$$

corresponding to problem (1.3), (1.4). It follows from Lemma 4.6 (see below) that the operator $\mathbf{L}: H_{a}^{l+2 m}(Q) \rightarrow \mathcal{H}_{a}^{l}(Q, \Gamma)$ is bounded.

The main result of this section is as follows. 
Theorem 4.1. Let Conditions 1.1 1.4 and 4.1 hold. Assume that the line $\operatorname{Im} \lambda=$ $a+1-l-2 m$ contains no eigenvalues of $\hat{\mathcal{L}}_{g}(\lambda)$ for any $g \in K$ and $\operatorname{dim} \mathcal{N}\left(\mathcal{L}_{g}(\omega)\right)=$ $\operatorname{codim} \mathcal{R}\left(\mathcal{L}_{g}(\omega)\right)=0$ for any $g \in K$ and $\omega \in S^{n-3}$. Then the following estimate holds for all $u \in H_{a}^{l+2 m}(Q)$ :

$$
\|u\|_{H_{a}^{l+2 m}(Q)} \leq c\left(\|\mathbf{L} u\|_{\mathcal{H}_{a}^{l}(Q, \Gamma)}+\|u\|_{H_{a}^{l+2 m-1}(Q)}\right),
$$

where $c>0$ does not depend on $u$.

Let $A^{0}$ and $B_{i \mu s}^{0}$ denote the principal homogeneous parts of the operators $A(x, D)$ and $B_{i \mu s}(x, D)$, respectively. Set

$$
B_{i \mu}^{0} u=\left.B_{i \mu 0}^{0} u\right|_{\Gamma_{i}} .
$$

For each $\varepsilon>0$, we introduce a function $\xi=\xi_{\varepsilon} \in C_{0}^{\infty}\left(\mathbb{R}^{n}\right)$ such that $\xi(x)=1$ for $x \in \mathcal{K}_{1}^{\varepsilon / 2}, \operatorname{supp} \xi(x) \subset \mathcal{K}_{1}^{\varepsilon}$, and

$$
\left|D^{\beta} \xi(x)\right| \leq k_{1} \varepsilon^{-|\beta|}, \quad x \in Q,
$$

where $k_{1}=k_{1}(\beta)>0$ does not depend on $\varepsilon$. Since $\omega_{i s}$ are $C^{\infty}$ diffeomorphisms, it follows that

$$
\operatorname{supp} \xi\left(\omega_{i s}(x)\right) \subset \mathcal{K}_{1}^{\varepsilon^{\prime \prime}}
$$

where $\varepsilon^{\prime \prime}=\varepsilon^{\prime \prime}(i, s, \varepsilon) \rightarrow 0$ as $\varepsilon \rightarrow 0\left(i=1, \ldots, N_{1} ; s=0, \ldots, S_{i}\right)$.

We assume that $\varepsilon>0$ is so small that

$$
0<\varepsilon^{\prime \prime}<\operatorname{dist}\left(\mathcal{K}_{1}, \mathcal{K}_{2} \cup \mathcal{K}_{3}\right) / 4
$$

Later on, we will make additional assumptions concerning $\varepsilon$ (see the proofs of Lemmas 4.3 and 5.2 .

Consider the operators

$$
\begin{aligned}
B_{i \mu}^{1} u & =\left.\sum_{s=1}^{S_{i}}\left(B_{i \mu s}^{0}(x, D)(\xi u)\right)\left(\omega_{i s}(x)\right)\right|_{\Gamma_{i}}, \\
B_{i \mu}^{2} u & =\left.\sum_{s=1}^{S_{i}}\left(B_{i \mu s}^{0}(x, D)((1-\xi) u)\right)\left(\omega_{i s}(x)\right)\right|_{\Gamma_{i}}, \\
B_{i \mu}^{3} & =B_{i \mu}-B_{i \mu}^{1}-B_{i \mu}^{2}, \quad A^{1}=A-A^{0} .
\end{aligned}
$$

The operators $B_{i \mu}^{1}$ correspond to nonlocal terms supported near the set $\mathcal{K}_{1}$ and the operators $B_{i \mu}^{2}$ to nonlocal terms supported outside the set $\mathcal{K}_{1}$, while $B_{i \mu}^{3}$ and $A^{1}$ correspond to lower-order terms (compact perturbations).

Denote $B^{k}=\left\{B_{i \mu}^{k}\right\}_{i, \mu}, k=0, \ldots, 3, B=B^{0}+\cdots+B^{3}$, and $C=B^{0}+B^{1}$.

Along with the operator $\mathbf{L}=(A, B)$ we consider the bounded operators

$$
\mathbf{L}^{0}=\left(A^{0}, B^{0}\right): H_{a}^{l+2 m}(Q) \rightarrow \mathcal{H}_{a}^{l}(Q, \Gamma), \quad \mathbf{L}^{1}=\left(A^{0}, C\right): H_{a}^{l+2 m}(Q) \rightarrow \mathcal{H}_{a}^{l}(Q, \Gamma) .
$$

We first obtain an a priori estimate (similar to (4.5)) for the operator $\mathbf{L}^{1}$ with sufficiently small $\varepsilon$. Then we prove a fundamental property of the operators $B_{i \mu}^{2}$ related to the fact that the operators $B_{i \mu}^{2}$ correspond to nonlocal terms supported outside the set $\mathcal{K}_{1}$. Combining these results will allow us to prove Theorem 4.1

Lemma 4.3. Let the conditions of Theorem 4.1 be fulfilled. Then there is an $\varepsilon>0$ such that the following estimate holds for all $u \in H_{a}^{l+2 m}(Q)$ :

$$
\|u\|_{H_{a}^{l+2 m}(Q)} \leq c\left(\left\|\mathbf{L}^{1} u\right\|_{\mathcal{H}_{a}^{l}(Q, \Gamma)}+\|u\|_{H_{a}^{l+2 m-1}(Q)}\right),
$$

where $c>0$ does not depend on $u$. 
Proof. 1. For any point $g \in \mathcal{K}_{1}$, denote by $\mathcal{O}(g)$ the orbit of $g$; see Sec. 1.1. By Condition 1.3, each orbit $\mathcal{O}(g)$ consists of finitely many points $g_{j}, j=1, \ldots, N(g)$. Set

$$
\chi_{m}=\chi_{m}(g)=\min _{j, \rho, k, s} \chi_{j \rho k s} \quad\left(j, k=1, \ldots, N(g) ; \rho=1,2 ; s=0,1, \ldots, S_{j \rho k}\right) .
$$

Clearly, $\chi_{m} \leq 1$. Let $x^{\prime} \rightarrow x(g, j)$ be the change of variables inverse to the change of variables $x \rightarrow x^{\prime}(g, j)$ from Sec. 1.1. The transformation $x^{\prime} \rightarrow x(g, j)$ takes each ball $B_{\chi_{m} \delta}$ onto some neighborhood $\hat{B}_{\delta}\left(g_{j}\right)$ of the point $g_{j}$ in such a way that the diameter of $\hat{B}_{\delta}\left(g_{j}\right)$ tends to zero as $\delta \rightarrow 0$. (Note that $\hat{B}_{\delta}\left(g_{j}\right)$ need not be a ball.) For each orbit $\mathcal{O}(g)$, we take a sufficiently small number $\delta=\delta(g)>0$ such that $\hat{B}_{\delta}\left(g_{j}\right) \subset \hat{V}\left(g_{j}\right), j=$ $1, \ldots, N(g)$, and the operator $\mathcal{L}_{g}^{\prime \prime}$ has a bounded inverse for $\delta=\delta(g)$ (see Corollary 2.1).

It is clear that the union

$$
\bigcup_{g \in \mathcal{K}_{1}} \bigcup_{j=1}^{N(g)} \hat{B}_{\delta(g)}\left(g_{j}\right)
$$

covers the set $\mathcal{K}_{1}$. We choose finitely many points $g^{t} \in \mathcal{K}_{1}, t=1, \ldots, T$, such that

$$
\mathcal{K}_{1} \subset \bigcup_{t, j} \hat{B}_{\delta\left(g^{t}\right)}\left(g_{j}^{t}\right) .
$$

Let functions $\varphi_{j}^{t} \in C_{0}^{\infty}\left(\mathbb{R}^{n}\right)$ form a partition of unity for the set $\mathcal{K}_{1}$ subordinate to the covering $\left\{\hat{B}_{\delta\left(g^{t}\right)}\left(g_{j}^{t}\right)\right\}$. Now we will pass from the partition of unity $\left\{\varphi_{j}^{t}\right\}$ to another partition of unity $\left\{\xi_{j}^{t}\right\}$ such that each function $\xi_{j}^{t}$, being written in the local coordinates $x^{\prime}=\left(y^{\prime}, z^{\prime}\right)$, does not depend on $y^{\prime}$ in a neighborhood of the edge $\mathcal{P}$. To do so, we denote the function $\varphi_{j}^{t}(x)$ written in the variables $x^{\prime}=\left(y^{\prime}, z^{\prime}\right)$ by $\varphi_{j}^{t}\left(y^{\prime}, z^{\prime}\right)$. Clearly, there is a number $a^{\prime}<\chi_{m} \delta\left(g^{t}\right)$ such that

$$
\varphi_{j}^{t}\left(0, z^{\prime}\right)=0
$$

for $a^{\prime}<\left|z^{\prime}\right|<\chi_{m} \delta\left(g^{t}\right)$. We can assume without loss of generality that the function $\varphi_{j}^{t}\left(0, z^{\prime}\right)$ is extended by zero for $\left|z^{\prime}\right| \geq \chi_{m} \delta\left(g^{t}\right)$, and it remains infinitely differentiable.

Denote by $\psi^{t} \in C_{0}^{\infty}\left(\mathbb{R}^{2}\right)$ a function such that $\psi^{t}\left(y^{\prime}\right)=1$ for $\left|y^{\prime}\right|<\varepsilon_{1}^{\prime}$ and $\psi_{j}^{t}\left(y^{\prime}\right)=0$ for $\left|y^{\prime}\right|>2 \varepsilon_{1}^{\prime}$, where $\varepsilon_{1}^{\prime}>0$ is so small that

$$
\left\{\left(2 \varepsilon_{1}^{\prime}\right)^{2}+\left(a^{\prime}\right)^{2}\right\}^{1 / 2}<\chi_{m} \delta\left(g^{t}\right),
$$

and $\varepsilon_{1}^{\prime}$ does not depend on $\varepsilon$.

Set

$$
\xi_{j}^{t}\left(y^{\prime}, z^{\prime}\right)=\psi^{t}\left(y^{\prime}\right) \varphi_{j}^{t}\left(0, z^{\prime}\right)
$$

By virtue of (4.10), we have

$$
\operatorname{supp} \xi_{j}^{t}\left(y^{\prime}, z^{\prime}\right) \subset B_{\chi_{m} \delta\left(g^{t}\right)} .
$$

It is also clear that

$$
\xi_{j}^{t}\left(y^{\prime}, z^{\prime}\right)=\varphi_{j}^{t}\left(0, z^{\prime}\right), \quad\left|y^{\prime}\right|<\varepsilon_{1}^{\prime} .
$$

Denote by $\xi_{j}^{t}(x)$ the functions $\xi_{j}^{t}\left(y^{\prime}, z^{\prime}\right)$ written in the variables $x=x\left(g^{t}, j\right)$. Since $\operatorname{supp} \xi_{j}^{t}(x) \subset \hat{B}_{\delta\left(g^{t}\right)}\left(g_{j}^{t}\right)$, we can extend each function $\xi_{j}^{t}(x)$ by zero outside the neighborhood $\hat{B}_{\delta\left(g^{t}\right)}\left(g_{j}^{t}\right)$ to obtain the function infinitely differentiable on $\mathbb{R}^{n}$.

Obviously, we have

$$
\sum_{j, t} \xi_{j}^{t}(x)=\sum_{j, t} \varphi_{j}^{t}(x)=1, \quad x \in \mathcal{K}_{1} .
$$

2. Take an arbitrary function $u \in H_{a}^{l+2 m}(Q)$. If $g_{j}^{t} \in \overline{\Gamma_{i}}$ and $x \in \hat{V}\left(g_{j}^{t}\right)$, then it follows from Condition 1.3 that $\omega_{i s}(x) \in V\left(g_{p}^{t}\right)$ for some $1 \leq p \leq N\left(g^{t}\right)$. Denote $u_{p}^{t}(x)=u(x)$ 
for $x \in Q \cap V\left(g_{p}^{t}\right)$. Then $u_{p}^{t}\left(\omega_{i s}(x)\right)=u\left(\omega_{i s}(x)\right)$ for $x \in Q \cap \hat{V}\left(g_{j}^{t}\right)$. Let $x \rightarrow x^{\prime}\left(g^{t}, j\right)$ be the change of variables from Sec. 1.1, corresponding to the orbit $\mathcal{O}\left(g^{t}\right)$. Denote the functions $\xi_{j}^{t}$ and $u_{j}^{t}$ written in the new variables $x^{\prime}$ by the same symbols (which leads to no confusion) and let $u^{t}=\left(u_{1}^{t}, \ldots, u_{N\left(g^{t}\right)}^{t}\right)$. Applying Corollary 2.1, we obtain

$$
\left\|\xi_{q}^{t} u\right\|_{H_{a}^{l+2 m}(Q)} \leq k_{1}\left\|\xi_{q}^{t} u_{q}^{t}\right\|_{H_{a}^{l+2 m}\left(\Theta_{q}\right)} \leq k_{1}\left\|\xi_{q}^{t} u^{t}\right\|_{\mathcal{H}_{a}^{l+2 m}(\Theta)} \leq k_{2}\left\|\mathcal{L}_{g^{t}}^{\prime \prime}\left(\xi_{q}^{t} u^{t}\right)\right\|_{\mathcal{H}_{a}^{l}(\Theta, \Gamma)},
$$

where $q=1, \ldots, N\left(g^{t}\right)$, while $k_{1}, k_{2}, \ldots>0$ do not depend on $u$.

It follows from (4.11) that

$$
\xi_{q}^{t}\left(\mathcal{G}_{j \rho k s} y^{\prime}, z^{\prime}\right)=0, \quad\left|x^{\prime}\right|>\delta\left(g^{t}\right) .
$$

Therefore, $\mathcal{L}_{g^{t}}^{\prime \prime}\left(\xi_{q}^{t} u^{t}\right)=\mathcal{L}_{g^{t}}^{\prime}\left(\xi_{q}^{t} u^{t}\right)$, and, by using Leibniz' formula, we have

$$
\begin{aligned}
\left\|\xi_{q}^{t} u\right\|_{H_{a}^{l+2 m}(Q)} \leq & k_{2}\left\|\mathcal{L}_{g^{t}}^{\prime}\left(\xi_{q}^{t} u^{t}\right)\right\|_{\mathcal{H}_{a}^{l}(\Theta, \Gamma)} \\
\leq & k_{3}\left(\left\|\mathbf{L}^{1} u\right\|_{\mathcal{H}_{a}^{l}(Q, \Gamma)}+\|u\|_{H_{a}^{l+2 m-1}(Q)}\right. \\
& \left.\quad+\sum_{h=1,2} \sum_{j, \rho, \mu} \sum_{(k, s) \neq(j, 0)}\left\|\Psi_{j \rho \mu k s}^{h}\right\|_{H_{a}^{l+2 m-m_{j \rho \mu}-1 / 2}\left(\Gamma_{j \rho}\right)}\right),
\end{aligned}
$$

where

$$
\begin{gathered}
\Psi_{j \rho \mu k s}^{1}=\left.\left(B_{j \rho \mu k s}^{0}\left(x^{\prime}, D_{y^{\prime}}, D_{z^{\prime}}\right)\left((1-\xi) \xi_{q}^{t} u_{k}^{t}\right)\right)\left(\mathcal{G}_{j \rho k s} y^{\prime}, z^{\prime}\right)\right|_{\Gamma_{j \rho},}, \\
\Psi_{j \rho \mu k s}^{2}=\left.\left(\xi_{q}^{t}\left(\mathcal{G}_{j \rho k s} y^{\prime}, z^{\prime}\right)-\xi_{q}^{t}\left(y^{\prime}, z^{\prime}\right)\right)\left(B_{j \rho \mu k s}^{0}\left(x^{\prime}, D_{y^{\prime}}, D_{z^{\prime}}\right)\left(\xi u_{k}^{t}\right)\right)\left(\mathcal{G}_{j \rho k s} y^{\prime}, z^{\prime}\right)\right|_{\Gamma_{j \rho}} .
\end{gathered}
$$

Denote by $\xi_{q k}^{t}(x)$ the function $\xi_{q}^{t}\left(y^{\prime}, z^{\prime}\right)$ written in the variables $x=x\left(g^{t}, k\right)$. Clearly, $\operatorname{supp} \xi_{q k}^{t} \subset \hat{B}_{\delta\left(g^{t}\right)}\left(g_{k}^{t}\right)$. Passing to the variables $\hat{x}=\omega_{i s}(x)$, we estimate the norm of $\Psi_{j \rho \mu k s}^{1}$ in the following way:

$$
\begin{aligned}
\left\|\Psi_{j \rho \mu k s}^{1}\right\|_{H_{a}^{l+2 m-m_{j \rho \mu}-1 / 2}\left(\Gamma_{j \rho}\right)} & \leq k_{4}\left\|\left.B_{i \mu s}^{0}(\hat{x}, D)\left((1-\xi) \xi_{q k}^{t} u\right)(\hat{x})\right|_{\omega_{i s}\left(\Gamma_{i}\right)}\right\|_{H_{a}^{l+2 m-m_{i \mu}-1 / 2}\left(\omega_{i s}\left(\Gamma_{i}\right)\right)} .
\end{aligned}
$$

Denote

$$
Q_{b}=\{x \in Q: \operatorname{dist}(x, \partial Q)>b\},
$$

where $b>0$. Since $\hat{B}_{\delta\left(g^{t}\right)}\left(g_{k}^{t}\right) \subset \hat{V}\left(g_{k}^{t}\right)$, it follows from Condition 1.3 that the set

$$
\Omega_{0}=\left(\overline{\omega_{i s}\left(\Gamma_{i}\right)} \cap \hat{B}_{\delta\left(g^{t}\right)}\left(g_{k}^{t}\right)\right) \backslash \mathcal{K}_{1}^{\varepsilon / 2}
$$

intersects neither $\mathcal{K}_{1}$ nor $\mathcal{K}_{2}$. Therefore, there exists a number $b=b(\varepsilon)>0$ such that $\Omega_{0} \subset Q_{b}$. Since

$$
\left.\operatorname{supp}\left((1-\xi) \xi_{q k}^{t}\right)\right|_{\omega_{i s}\left(\Gamma_{i}\right)} \subset \Omega_{0} \subset Q_{b},
$$

using the last inequality, the equivalence of the norms in the spaces $H_{a}^{l+2 m}\left(Q_{b}\right)$ and $W^{l+2 m}\left(Q_{b}\right)$, and Lemma 3.5 , we obtain

$$
\begin{aligned}
& \left\|\Psi_{j \rho \mu k s}^{1}\right\|_{H_{a}^{l+2 m-m_{j \rho \mu}-1 / 2}\left(\Gamma_{j \rho}\right)} \leq k_{5}\|u\|_{H_{a}^{l+2 m}\left(Q_{b}\right)} \leq k_{6}\|u\|_{W^{l+2 m}\left(Q_{b}\right)} \\
& \quad \leq k_{7}\left(\left\|A^{0} u\right\|_{W^{l}\left(Q_{b / 2}\right)}+\|u\|_{L_{2}\left(Q_{b / 2}\right)}\right) \leq k_{8}\left(\left\|A^{0} u\right\|_{H_{a}^{l}(Q)}+\|u\|_{H_{a}^{l+2 m-1}(Q)}\right) .
\end{aligned}
$$

Now let us estimate the norm of $\Psi_{j \rho \mu k s}^{2}$. By virtue of (4.12),

$$
\xi_{q}^{t}\left(\mathcal{G}_{j \rho k s} y^{\prime}, z^{\prime}\right)-\xi_{q}^{t}\left(y^{\prime}, z^{\prime}\right)=0, \quad\left|y^{\prime}\right|<\varepsilon_{1}^{\prime} / \max \left(1, \chi_{j \rho k s}\right) .
$$

Therefore, similarly to (4.15), we obtain

$$
\left\|\Psi_{j \rho \mu k s}^{2}\right\|_{H_{a}^{l+2 m-m_{j \rho \mu}-1 / 2}\left(\Gamma_{j \rho}\right)} \leq k_{9}\left(\left\|A^{0} u\right\|_{H_{a}^{l}(Q)}+\|u\|_{H_{a}^{l+2 m-1}(Q)}\right) .
$$


It follows from (4.14), (4.15), and (4.16) that

$$
\left\|\xi_{q}^{t} u\right\|_{H_{a}^{l+2 m}(Q)} \leq k_{10}\left(\left\|\mathbf{L}^{1} u\right\|_{\mathcal{H}_{a}^{l}(Q, \Gamma)}+\|u\|_{H_{a}^{l+2 m-1}(Q)}\right) .
$$

Setting

$$
\xi_{0}(x)=\sum_{t, q} \xi_{q}^{t}(x)
$$

and using inequality (4.18), we have

$$
\left\|\xi_{0} u\right\|_{H_{a}^{l+2 m}(Q)} \leq k_{11}\left(\left\|\mathbf{L}^{1} u\right\|_{\mathcal{H}_{a}^{l}(Q, \Gamma)}+\|u\|_{H_{a}^{l+2 m-1}(Q)}\right)
$$

(note that $k_{11}$ depends on $\varepsilon$ ).

3. Using a partition of unity, Theorem 2.3 . Leibniz' formula, and a priori estimates of solutions for elliptic problems in the interior of $Q$ and near a smooth part of the boundary, we obtain

$$
\begin{aligned}
\left\|\left(1-\xi_{0}\right) u\right\|_{H_{a}^{l+2 m}(Q) \leq} \leq & c_{1}\left(\left\|\left(1-\xi_{0}\right) \mathbf{L}^{0} u\right\|_{\mathcal{H}_{a}^{l}(Q, \Gamma)}+\|u\|_{H_{a}^{l+2 m-1}(Q)}\right) \\
\leq & c_{2}\left(\left\|\left(1-\xi_{0}\right) \mathbf{L}^{1} u\right\|_{\mathcal{H}_{a}^{l}(Q, \Gamma)}\right. \\
& \left.\quad+\sum_{i, \mu}\left\|\left(1-\xi_{0}\right) B_{i \mu}^{1} u\right\|_{H_{a}^{l+2 m-m_{i \mu}-1 / 2}\left(\Gamma_{i}\right)}+\|u\|_{H_{a}^{l+2 m-1}(Q)}\right),
\end{aligned}
$$

where $c_{1}, c_{2}, \ldots>0$ do not depend on $u$ and $\varepsilon$ (we recall that the function $\xi_{0}$ does not depend on $\varepsilon$ ).

It follows from (4.13) and (4.18) that $1-\xi_{0}(x)=0$ for $x \in \mathcal{K}_{1}$. On the other hand, $\operatorname{supp} \xi\left(\omega_{i s}(x)\right) \subset \mathcal{K}_{1}^{\varepsilon^{\prime \prime}}$ due to (4.7). Therefore, applying Lemma 4.1 and Remark 4.1 and taking into account that $\omega_{i s}$ are $C^{\infty}$ diffeomorphisms, we have

$$
\left\|\left(1-\xi_{0}\right) B_{i \mu}^{1} u\right\|_{H_{a}^{l+2 m-m_{i \mu}-1 / 2}\left(\Gamma_{i}\right)} \leq c_{3} \varepsilon^{\prime \prime}\left\|B_{i \mu}^{1} u\right\|_{H_{a}^{l+2 m-m_{i \mu}-1 / 2}\left(\Gamma_{i}\right)} \leq c_{4} \varepsilon^{\prime \prime}\|\xi u\|_{H_{a}^{l+2 m}(Q)} .
$$

Further, using the relation $\operatorname{supp} \xi \subset \mathcal{K}_{1}^{\varepsilon}$, inequalities (4.6), and Lemma 4.2, we obtain from the last estimate that

$$
\left\|\left(1-\xi_{0}\right) B_{i \mu}^{1} u\right\|_{H_{a}^{l+2 m-m_{i \mu}-1 / 2}\left(\Gamma_{i}\right)} \leq c_{5} \varepsilon^{\prime \prime}\|u\|_{H_{a}^{l+2 m}(Q)} .
$$

Combining this estimate with (4.20) yields

$$
\left\|\left(1-\xi_{0}\right) u\right\|_{H_{a}^{l+2 m}(Q)} \leq c_{6}\left(\left\|\mathbf{L}^{1} u\right\|_{\mathcal{H}_{a}^{l}(Q, \Gamma)}+\varepsilon^{\prime \prime}\|u\|_{H_{a}^{l+2 m}(Q)}+\|u\|_{H_{a}^{l+2 m-1}(Q)}\right) .
$$

Choosing $\varepsilon$ in the definition of the function $\xi$ so small that $c_{6} \varepsilon^{\prime \prime}=1 / 2$ and using inequalities (4.19) and (4.21), we complete the proof.

4.3. In this subsection, we prove Theorem 4.1. First, we formulate some results on properties of weighted spaces, which are needed below.

Lemma 4.4. Let $Q_{1} \subset \mathbb{R}^{n}$ be a bounded domain such that $\bar{Q} \subset Q_{1}$. Assume that the set $K_{1}$ in the definition of the space $H_{a}^{k}\left(Q_{1}\right)=H_{a}^{k}\left(Q_{1}, K_{1}\right)$ coincides with the set $K$ in the definition of the space $H_{a}^{k}(Q)=H_{a}^{k}(Q, K)$. Then, for any function $v \in H_{a}^{k}(Q)$, there exists a function $v_{1} \in H_{a}^{k}\left(Q_{1}\right)$ such that $v_{1}(x)=v(x)$ for $x \in Q$ and

$$
\left\|v_{1}\right\|_{H_{a}^{k}\left(Q_{1}\right)} \leq c\|v\|_{H_{a}^{k}(Q)},
$$

where $c>0$ does not depend on $v$.

Lemma 4.4 is proved in [27, Sec. 3].

Lemma 4.5. Let $a>l+2 m-1$. Assume that $\delta>0$ satisfies the following conditions:

(1) $\mathcal{K}_{1}^{\delta} \cap\left(\mathcal{K}_{2} \cup \mathcal{K}_{3}\right)=\varnothing$ if $\mathcal{K}_{2} \cup \mathcal{K}_{3} \neq \varnothing$, 
(2) $\mathcal{K}_{2}^{\delta} \cap\left(\mathcal{K} \backslash \mathcal{K}_{2}\right)=\varnothing$ if $\mathcal{K}_{2} \neq \varnothing$,

(3) $\mathcal{K}_{3}^{\delta} \subset Q$ and $\mathcal{K}_{3}^{\delta} \cap\left(\mathcal{K} \backslash \mathcal{K}_{3}\right)=\varnothing$ if $\mathcal{K}_{3} \neq \varnothing$,

(4) $\delta>0$ is arbitrary if $\mathcal{K}_{2} \cup \mathcal{K}_{3}=\varnothing$.

Then

$$
\|u\|_{H_{a}^{l+2 m}\left(\mathcal{K}_{j}^{\delta} \cap Q\right)} \leq c_{1}\|u\|_{W^{l+2 m}\left(\mathcal{K}_{j}^{\delta} \cap Q\right)}
$$

for all $u \in W^{l+2 m}\left(\mathcal{K}_{j}^{\delta} \cap Q\right)$ if $\mathcal{K}_{j} \neq \varnothing(j=1,2)$ and

$$
\|u\|_{H_{a}^{l+2 m}\left(\mathcal{K}_{3}^{\delta}\right)} \leq c_{2}\|u\|_{W^{l+2 m}\left(\mathcal{K}_{3}^{\delta}\right)}
$$

for all $u \in W^{l+2 m}\left(\mathcal{K}_{3}^{\delta}\right)$ if $\mathcal{K}_{3} \neq \varnothing$, where $c_{1}, c_{2}>0$ do not depend on $u$.

Lemma 4.5] is proved in 27] (see also Lemma 5.2 in [17]).

The following result is also obtained in [27, Sec. 3]. It means that the operators $B_{i \mu}^{2}$ correspond to nonlocal terms supported outside the set $\mathcal{K}_{1}$. For the reader's convenience, we give the proof of this result.

Lemma 4.6. Let Condition 4.1 hold. Then there exists a number $\varkappa=\varkappa(\varepsilon)>0$ such that

$$
\left\|B_{i \mu}^{2} u\right\|_{H_{a}^{l+2 m-m_{i \mu}-1 / 2}\left(\Gamma_{i}\right)} \leq c_{1}\|u\|_{H_{a}^{l+2 m}\left(Q \backslash \overline{\mathcal{K}_{1}^{2 \varkappa}}\right)}
$$

for all $u \in H_{a}^{l+2 m}\left(Q \backslash \overline{\mathcal{K}_{1}^{2 \varkappa}}\right)$; furthermore, there exists a number $\sigma=\sigma(\varkappa)$ such that

$$
\left\|B_{i \mu}^{2} u\right\|_{H_{a}^{l+2 m-m_{i \mu}-1 / 2}\left(\Gamma_{i} \backslash \overline{\mathcal{K}_{1}^{\varkappa}}\right)} \leq c_{2}\|u\|_{H_{a}^{l+2 m}\left(Q_{\sigma}\right)}
$$

for all $u \in H_{a}^{l+2 m}\left(Q_{\sigma}\right)$; here $i=1, \ldots, N_{0} ; \mu=1, \ldots, m ; c_{1}, c_{2}>0$ do not depend on $u$.

Proof. 1. It suffices to show that inequalities (4.24) and (4.25) are valid for the function

$$
\varphi_{i \mu s}=\left.\left(B_{i \mu s}^{0}(x, D)((1-\xi) u)\right)\left(\omega_{i s}(x)\right)\right|_{\Gamma_{i}}
$$

substituted for $B_{i \mu}^{2} u$.

2. Let $\overline{\omega_{i s}\left(\Gamma_{i}\right)} \cap \mathcal{K}_{2} \neq \varnothing$. We assume without loss of generality that $\overline{\omega_{i s}\left(\Omega_{i}\right)} \cap \mathcal{K}_{2}=$ $\overline{\omega_{i s}\left(\Gamma_{i}\right)} \cap \mathcal{K}_{2}$; see Figure 3, Let $U$ be an extension of the function $(1-\xi) u$ to $Q \cup \omega_{i s}\left(\Omega_{i}\right)$,

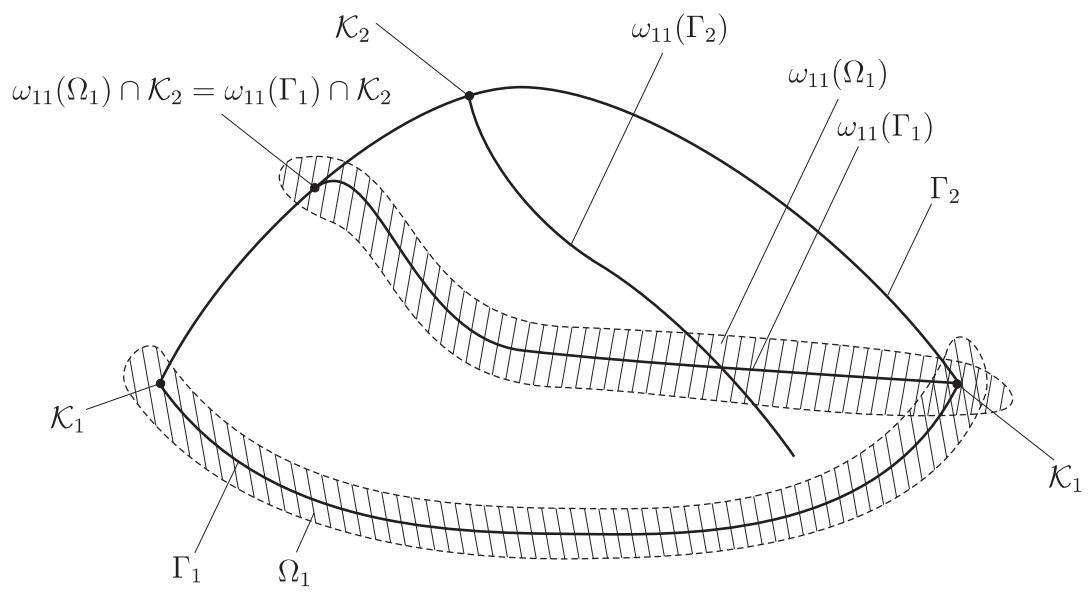

Figure 3 . The domain $Q$

defined by Lemma 4.4 and satisfying the inequality

$$
\|U\|_{H_{a}^{l+2 m}\left(Q \cup \omega_{i s}\left(\Omega_{i}\right)\right)} \leq k_{1}\|(1-\xi) u\|_{H_{a}^{l+2 m}(Q)},
$$


where $k_{1}, k_{2}, \ldots>0$ do not depend on $u$. Set

$$
\Phi_{i \mu s}(x)=\left(B_{i \mu s}^{0}(x, D) U\right)\left(\omega_{i s}(x)\right), \quad x \in \Omega_{i} .
$$

Clearly,

$$
\varphi_{i \mu s}=\left.\Phi_{i \mu s}\right|_{\Gamma_{i}} .
$$

Introducing the new variable $\hat{x}=\omega_{i s}(x)$, applying Lemma 4.5 if $\mathcal{K}_{j} \neq \varnothing$ and $\mathcal{K}_{j} \not \subset K$, $j=2,3$, and using inequality (4.26), we obtain

$$
\begin{aligned}
& \left\|\varphi_{i \mu s}\right\|_{H_{a}^{l+2 m-m_{i \mu}-1 / 2}\left(\Gamma_{i}\right)} \leq\left\|\Phi_{i \mu s}\right\|_{H_{a}^{l+2 m-m_{i \mu}}\left(\Omega_{i}\right)} \\
& \quad \leq k_{2}\left\|B_{i \mu s}^{0}(\hat{x}, D) U(\hat{x})\right\|_{H_{a}^{l+2 m-m_{i \mu}}\left(\omega_{i s}\left(\Omega_{i}\right)\right)} \leq k_{3}\|(1-\xi) u\|_{H_{a}^{l+2 m}(Q)} .
\end{aligned}
$$

Thus, setting $2 \varkappa=\varepsilon / 2$, we see that (4.27) implies (4.24).

Since the transformation $\omega_{i s}$ is continuous and $\omega_{i s}\left(\Gamma_{i}\right) \subset Q$, it follows that $\omega_{i s}\left(\Gamma_{i} \backslash\right.$ $\left.\overline{\mathcal{K}_{1}^{\varkappa}}\right) \subset Q_{2 \sigma}$ for sufficiently small $\sigma>0$. Introduce a function $\eta \in C_{0}^{\infty}\left(\mathbb{R}^{n}\right)$ such that $\eta(x)=1$ for $x \in Q_{2 \sigma}$ and $\eta(x)=0$ for $x \notin Q_{\sigma}$.

Suppose that the function $\eta u$ is extended by zero outside $Q$. Set

$$
\Psi_{i \mu s}(x)=\left(B_{i \mu s}^{0}(x, D)(\eta(1-\xi) u)\right)\left(\omega_{i s}(x)\right), \quad x \in \Omega_{i} .
$$

It is clear that

$$
\left.\varphi_{i \mu s}\right|_{\Gamma_{i} \backslash \overline{\mathcal{K}_{1}^{\varkappa}}}=\left.\Psi\right|_{\Gamma_{i} \backslash \overline{\mathcal{K}_{1}^{\varkappa}}} .
$$

Hence, applying Lemma 4.5 if $\mathcal{K}_{j} \neq \varnothing$ and $\mathcal{K}_{j} \not \subset K, j=2,3$, we obtain

$$
\begin{aligned}
& \left\|\varphi_{i \mu s}\right\|_{H_{a}^{l+2 m-m_{i \mu}-1 / 2}\left(\Gamma_{i} \backslash \overline{\mathcal{K}_{1}^{\varkappa}}\right)} \leq\left\|\Psi_{i \mu s}\right\|_{H_{a}^{l+2 m-m_{i \mu}}\left(\Omega_{i}\right)} \\
& \quad \leq k_{4}\left\|B_{i \mu s}^{0}(\hat{x}, D)(\eta(1-\xi) u)(\hat{x})\right\|_{H_{a}^{l+2 m-m_{i \mu}}\left(\omega_{i s}\left(\Omega_{i}\right)\right)} \leq k_{5}\|u\|_{H_{a}^{l+2 m}\left(Q_{\sigma}\right)} .
\end{aligned}
$$

3. If $\overline{\omega_{i s}\left(\Gamma_{i}\right)} \cap \mathcal{K}_{2}=\varnothing$, then $\overline{\omega_{i s}\left(\Gamma_{i}\right)} \backslash \mathcal{K}_{1}^{\varepsilon / 2} \subset Q$. Therefore, similarly to the above, we obtain

$$
\left\|\varphi_{i \mu s}\right\|_{H_{a}^{l+2 m-m_{i \mu}-1 / 2}\left(\Gamma_{i}\right)} \leq k_{5}\|u\|_{H_{a}^{l+2 m}\left(Q_{\sigma}\right)} .
$$

This proves inequalities (4.24) and (4.25).

Proof of Theorem 4.1. 1. Take an arbitrary function $u \in H_{a}^{l+2 m}(Q)$. It follows from Lemma 4.3 that

$$
\|u\|_{H_{a}^{l+2 m}(Q)} \leq k_{1}\left(\|\mathbf{L} u\|_{\mathcal{H}_{a}^{l}(Q, \Gamma)}+\left\|A^{1} u\right\|_{H_{a}^{l}(Q)}+\sum_{i, \mu} \sum_{k=2,3}\left\|B_{i \mu}^{k} u\right\|_{H_{a}^{l+2 m-m_{i \mu}-1 / 2}\left(\Gamma_{i}\right)}\right),
$$

where $k_{1}, k_{2}, \ldots>0$ do not depend on $u$.

It follows from the boundedness of the domain $Q$ and from Lemma 4.5 that

$$
\left\|A^{1} u\right\|_{H_{a}^{l}(Q)}+\sum_{i, \mu}\left\|B_{i \mu}^{3} u\right\|_{H_{a}^{l+2 m-m_{i \mu}-1 / 2}\left(\Gamma_{i}\right)} \leq k_{2}\|u\|_{H_{a}^{l+2 m-1}(Q)} .
$$

2. Consider a function $\eta \in C^{\infty}\left(\mathbb{R}^{n}\right)$ such that

$$
\eta(x)=1 \quad \text { for } x \in \mathbb{R}^{n} \backslash \overline{\mathcal{K}_{1}^{2 \varkappa}}, \quad \eta(x)=0 \quad \text { for } x \in \mathcal{K}_{1}^{\varkappa},
$$

where $\varkappa>0$ is the constant occurring in Lemma 4.6

It follows from inequality (4.24), from Lemma 4.3. and from Leibniz' formula that

$$
\begin{aligned}
& \left\|B_{i \mu}^{2} u\right\|_{H_{a}^{l+2 m-m_{i \mu}-1 / 2}\left(\Gamma_{i}\right)} \leq k_{3}\|\eta u\|_{H_{a}^{l+2 m}(Q)} \\
& \quad \leq k_{4}\left(\left\|\eta \mathbf{L}^{1} u\right\|_{\mathcal{H}_{a}^{l}(Q, \Gamma)}+\|u\|_{H_{a}^{l+2 m-1}(Q)}+\sum_{i, \mu} \sum_{s \neq 0}\left\|\Psi_{i \mu s}\right\|_{H_{a}^{l+2 m-m_{i \mu}-1 / 2}\left(\omega_{i s}\left(\Gamma_{i}\right)\right)}\right),
\end{aligned}
$$


where

$$
\Psi_{i \mu s}=\left.\left(\eta(x)-\eta\left(\omega_{i s}^{-1}(x)\right)\right)\left(B_{i \mu s}^{0}(x, D)(\xi u)\right)(x)\right|_{\omega_{i s}\left(\Gamma_{i}\right)} .
$$

It is clear that, if $\overline{\omega_{i s}\left(\Gamma_{i}\right)} \cap \mathcal{K}_{1}^{\varepsilon}=\varnothing$, then $\Psi_{i \mu s}=0$. Let $\overline{\omega_{i s}\left(\Gamma_{i}\right)} \cap \mathcal{K}_{1}^{\varepsilon} \neq \varnothing$. We claim that

$$
\operatorname{supp} \Psi_{i \mu s} \subset Q_{b}
$$

for some $b>0$. Indeed,

$$
\omega_{i s}\left(\Gamma_{i}\right) \subset Q \quad \text { and } \quad \operatorname{supp} \xi \subset \overline{\mathcal{K}_{1}^{\varepsilon}} .
$$

Therefore, by virtue of (4.8), it suffices to show that $\Psi_{i \mu s}(x)=0$ for $x$ in some neighborhood of $\mathcal{K}_{1}$. Since

$$
\eta(x)=0, \quad x \in \overline{\omega_{i s}\left(\Gamma_{i}\right)} \cap \mathcal{K}_{1}^{\varkappa},
$$

it remains to prove that

$$
\eta\left(\omega_{i s}^{-1}(x)\right)=0, \quad x \in \overline{\omega_{i s}\left(\Gamma_{i}\right)} \cap \mathcal{K}_{1}^{d},
$$

for a sufficiently small $d>0$. Note that, if $\overline{\omega_{i s}\left(\Gamma_{i}\right)} \cap \mathcal{K}_{1}=\varnothing$, then (4.31) follows from (4.8) and (4.32). If $\overline{\omega_{i s}\left(\Gamma_{i}\right)} \cap \mathcal{K}_{1} \neq \varnothing$ for some $i$ and $s$, then $\mathcal{K}_{1 \nu} \subset \overline{\omega_{i s}\left(\Gamma_{i}\right)}$ for some $\nu$ and $\omega_{i s}^{-1}\left(\mathcal{K}_{1 \nu}\right) \subset \mathcal{K}_{1}$. Hence, there exists a sufficiently small $d>0$ such that $\mathcal{K}_{1 \nu}^{d} \subset \omega_{i s}\left(\Omega_{i}\right)$ and $\omega_{i s}^{-1}\left(\mathcal{K}_{1 \nu}^{d}\right) \subset \mathcal{K}_{1}^{\varkappa}$ (because the transformations $\omega_{i s}^{-1}$ are smooth). Clearly, (4.34) holds in this case. Thus, we obtain (4.31).

It follows from (4.31) and Lemma 3.5 that

$$
\left\|\Psi_{i \mu s}\right\|_{H_{a}^{l+2 m-m_{i \mu}-1 / 2}\left(\omega_{i s}\left(\Gamma_{i}\right)\right)} \leq k_{5}\left(\|A u\|_{H_{a}^{l}(Q)}+\|u\|_{H_{a}^{l+2 m-1}(Q)}\right) .
$$

Using this inequality, we infer from (4.30) that

$$
\begin{aligned}
& \left\|B_{i \mu}^{2} u\right\|_{H_{a}^{l+2 m-m_{i \mu}-1 / 2}\left(\Gamma_{i}\right)}^{l+2} \\
& \quad \leq k_{6}\left(\|\mathbf{L} u\|_{\mathcal{H}_{a}^{l}(Q, \Gamma)}+\|u\|_{H_{a}^{l+2 m-1}(Q)}+\sum_{i, \mu}\left\|\eta B_{i \mu}^{2} u\right\|_{H_{a}^{l+2 m-m_{i \mu}-1 / 2}\left(\Gamma_{i}\right)}\right) .
\end{aligned}
$$

By virtue of (4.25), Lemma 3.5, and Leibniz' formula, we have

$$
\left\|\eta B_{i \mu}^{2} u\right\|_{H_{a}^{l+2 m-m_{i \mu}-1 / 2}\left(\Gamma_{i}\right)} \leq k_{7}\|u\|_{H_{a}^{l+2 m}\left(Q_{\sigma}\right)} \leq k_{8}\left(\|A u\|_{H_{a}^{l}(Q)}+\|u\|_{H_{a}^{l+2 m-1}(Q)}\right) .
$$

Combining estimates (4.28), (4.29), (4.35), and (4.36), we obtain the desired estimate (4.5).

\section{The Fredholm Property of NONLOCAL ELLIPTIC PROBlemS}

5.1. In this section, we prove the main result of the paper concerning the Fredholm property of nonlocal elliptic problems in weighted spaces. This result can be formulated as follows.

Theorem 5.1. Let Conditions 1.1 1.4 and 4.1 hold. Assume that the line $\operatorname{Im} \lambda=$ $a+1-l-2 m$ contains no eigenvalues of $\hat{\mathcal{L}}_{g}(\lambda)$ for any $g \in K$ and $\operatorname{dim} \mathcal{N}\left(\mathcal{L}_{g}(\omega)\right)=$ $\operatorname{codim} \mathcal{R}\left(\mathcal{L}_{g}(\omega)\right)=0$ for any $g \in K$ and $\omega \in S^{n-3}$. Then the operator $\mathbf{L}: H_{a}^{l+2 m}(Q) \rightarrow$ $\mathcal{H}_{a}^{l}(Q, \Gamma)$ has the Fredholm property.

Due to Theorem 16.4 in [18 (concerning compact perturbations of Fredholm operators), it suffices to prove Theorem 5.1 and the other assertions of this section for $A^{1}=0$ and $B^{3}=0$. Therefore, we assume that $A^{1}=0$ and $B^{3}=0$ throughout this section.

Corollary 5.1. Let the conditions of Theorem 5.1 be fulfilled. Then ind $\mathbf{L}=\operatorname{ind} \mathbf{L}^{1}$. 
Proof. We introduce the operator

$$
L_{t} u=\left\{A^{0} u, C u+(1-t) B^{2} u\right\} .
$$

We have $L_{0}=\mathbf{L}$ (because $A^{1}=0$ and $B^{3}=0$ ) and $L_{1}=\mathbf{L}^{1}$.

By Theorem 5.1 the operators $L_{t}$ have the Fredholm property for all $t$. Furthermore, for any $t_{0}$ and $t$, the following estimate holds:

$$
\left\|L_{t} u-L_{t_{0}} u\right\|_{\mathcal{H}_{a}^{l}(Q, \Gamma)} \leq k_{t_{0}}\left|t-t_{0}\right| \cdot\|u\|_{H_{a}^{l+2 m}(Q)},
$$

where $k_{t_{0}}>0$ does not depend on $t$. Therefore, by Theorem 16.2 in [18, we have ind $L_{t}=$ ind $L_{t_{0}}$ for any $t$ in a sufficiently small neighborhood of the point $t_{0}$. Since $t_{0}$ is arbitrary, these neighborhoods cover the segment $[0,1]$. Choosing a finite subcovering, we obtain the relations ind $\mathbf{L}=$ ind $L_{0}=$ ind $L_{1}=$ ind $\mathbf{L}^{1}$.

The proof of Theorem 5.1 is based on the existence of a right regularizer for the operator $\mathbf{L}$.

Theorem 5.2. Let the conditions of Theorem 5.1 be fulfilled. Then there exists a linear bounded operator $\mathbf{R}: \mathcal{H}_{a}^{l}(Q, \Gamma) \rightarrow H_{a}^{l+2 m}(Q)$ such that

$$
\mathbf{L R}=\mathbf{I}+\mathbf{T}
$$

where $\mathbf{I}$ and $\mathbf{T}$ are the identity operator and a compact operator on $\mathcal{H}_{a}^{l}(Q, \Gamma)$, respectively.

Proof of Theorem [5.1. Assume that Theorem [5.2 is true. By Lemma 3.5 in [15], the embedding of $H_{a}^{l+2 m}(Q)$ into $H_{a}^{l+2 m-1}(Q)$ is compact. Therefore, by Theorem 7.1 in [18] and Theorem 4.1 $\operatorname{dim} \mathcal{N}(\mathbf{L})<\infty$ and the range $\mathcal{R}(\mathbf{L})$ is closed in $\mathcal{H}_{a}^{l}(Q, \Gamma)$. On the other hand, Theorem 15.2 in [18] and Theorem 5.2 imply that $\operatorname{codim} \mathcal{R}(\mathbf{L})<\infty$.

Thus, it remains to prove Theorem 5.2 ,

5.2. First, we prove the following auxiliary result.

Lemma 5.1. Let $H$ be a Hilbert space and $I$ the identity operator on $H$. Let $M_{\varepsilon}$ and $S_{\varepsilon}, \varepsilon>0$, be families of bounded operators on $H$ such that

$$
\left\|M_{\varepsilon}\right\| \leq c_{1} \varepsilon, \quad\left\|S_{\varepsilon}\right\| \leq c_{2},
$$

where $c_{1}, c_{2}>0$ do not depend on $\varepsilon$, and the operators $S_{\varepsilon}^{2}$ are compact. Then the operators

$$
L_{\varepsilon}=I+M_{\varepsilon}+S_{\varepsilon}
$$

have the Fredholm property for sufficiently small $\varepsilon>0$.

Proof. To prove the lemma, we will construct a right and a left regularizer for $L_{\varepsilon}$. We have

$$
L_{\varepsilon}\left(I-\left(M_{\varepsilon}+S_{\varepsilon}\right)\right)=I-M_{\varepsilon}^{2}-M_{\varepsilon} S_{\varepsilon}-S_{\varepsilon} M_{\varepsilon}-S_{\varepsilon}^{2} .
$$

It follows from (5.1) that

$$
\left\|M_{\varepsilon}^{2}+M_{\varepsilon} S_{\varepsilon}+S_{\varepsilon} M_{\varepsilon}\right\| \leq c_{3} \varepsilon,
$$

where $c_{3}>0$ does not depend on $\varepsilon$. Therefore, the operators $I-M_{\varepsilon}^{2}-M_{\varepsilon} S_{\varepsilon}-S_{\varepsilon} M_{\varepsilon}$ have the Fredholm property by Theorem 16.2 in [18, provided that $\varepsilon>0$ is sufficiently small. Further, using the fact that the operators $S_{\varepsilon}^{2}$ are compact and applying Theorem 16.4 in [18, we see that the operators $L_{\varepsilon}\left(I-\left(M_{\varepsilon}+S_{\varepsilon}\right)\right)$ also have the Fredholm property. Now it follows from Theorem 15.2 in [18] that there exist bounded operators $R_{1 \varepsilon}$ and compact operators $T_{1 \varepsilon}$ such that

$$
L_{\varepsilon}\left(I-\left(M_{\varepsilon}+S_{\varepsilon}\right)\right) R_{1 \varepsilon}=I+T_{1 \varepsilon} .
$$


Similarly, one can prove that there exist bounded operators $R_{2 \varepsilon}$ and compact operators $T_{2 \varepsilon}$ such that

$$
R_{2 \varepsilon}\left(I-\left(M_{\varepsilon}+S_{\varepsilon}\right)\right) L_{\varepsilon}=I+T_{2 \varepsilon} .
$$

The conclusion of the lemma follows from relations (5.2) and (5.3) and from Theorems 15.2 and 14.3 in 18 .

To prove Theorem [5.2, we preliminarily consider the operator $\mathbf{L}^{1}$, i.e., assume that nonlocal terms are supported near the set $\mathcal{K}_{1}$.

Lemma 5.2. Let the conditions of Theorem 5.1 be fulfilled and the number $\varepsilon$ be sufficiently small. Then there exist a linear bounded operator $\mathbf{R}_{1}: \mathcal{H}_{a}^{l}(Q, \Gamma) \rightarrow H_{a}^{l+2 m}(Q)$ and a compact operator $\mathbf{T}_{1}: \mathcal{H}_{a}^{l}(Q, \Gamma) \rightarrow \mathcal{H}_{a}^{l}(Q, \Gamma)$ such that

$$
\mathbf{L}^{1} \mathbf{R}_{1}=\mathbf{I}+\mathbf{T}_{1} \text {. }
$$

Proof. 1. To construct a right regularizer, we consider a partition of unity $\left\{\xi_{j}^{t}\right\}$ different from that in the proof of Lemma 4.3

For each orbit $\mathcal{O}(g), g \in \mathcal{K}_{1}$, we denote by $\hat{B}_{\delta}\left(g_{j}\right)$ the same neighborhoods as in the proof of Lemma 4.3. and we let $\left\{\varphi_{j}^{t}\right\}$ be the same partition of unity for $\mathcal{K}_{1}$. We denote the function $\varphi_{j}^{t}(x)$ written in the variables $x^{\prime}=\left(y^{\prime}, z^{\prime}\right)$ by $\varphi_{j}^{t}\left(y^{\prime}, z^{\prime}\right)$. Clearly, there is a number $a^{\prime}<\chi_{m} \delta\left(g^{t}\right)$ such that

$$
\varphi_{j}^{t}\left(0, z^{\prime}\right)=0
$$

for $a^{\prime}<\left|z^{\prime}\right|<\chi_{m} \delta\left(g^{t}\right)$. As before, we assume that $\varphi_{j}^{t}\left(0, z^{\prime}\right)$ is extended by zero for $\left|z^{\prime}\right| \geq \chi_{m} \delta\left(g^{t}\right)$, and it remains infinitely differentiable.

Let $\hat{\varphi}^{t} \in C_{0}^{\infty}\left(\mathbb{R}^{n-2}\right)$ be a function such that

$$
\begin{array}{ll}
\hat{\varphi}^{t}\left(z^{\prime}\right)=1, & \left|z^{\prime}\right|<a^{\prime}, \\
\hat{\varphi}^{t}\left(z^{\prime}\right)=0, & \left|z^{\prime}\right|>\hat{a}^{\prime},
\end{array}
$$

where $a^{\prime}<\hat{a}^{\prime}<\chi_{m} \delta\left(g^{t}\right)$.

Denote by $\psi^{t}, \hat{\psi}^{t} \in C_{0}^{\infty}\left(\mathbb{R}^{2}\right)$ functions such that $\psi^{t}\left(y^{\prime}\right)=1$ for $\left|y^{\prime}\right| \leq \varepsilon_{1}^{\prime}$ and $\psi^{t}\left(y^{\prime}\right)=0$ for $\left|y^{\prime}\right| \geq 3 \varepsilon_{1}^{\prime} / 2, \hat{\psi}^{t}\left(y^{\prime}\right)=1$ for $\left|y^{\prime}\right| \leq 3 \varepsilon_{1}^{\prime} / 2$, and $\hat{\psi}^{t}\left(y^{\prime}\right)=0$ for $\left|y^{\prime}\right| \geq 2 \varepsilon_{1}^{\prime}$, where $\varepsilon_{1}^{\prime}>0$ is so small that

$$
\left\{\left(2 \varepsilon_{1}^{\prime}\right)^{2}+\left(\hat{a}^{\prime}\right)^{2}\right\}^{1 / 2}<\chi_{m} \delta\left(g^{t}\right),
$$

and $\varepsilon_{1}^{\prime}$ does not depend on $\varepsilon$.

Set

$$
\xi_{j}^{t}\left(y^{\prime}, z^{\prime}\right)=\psi^{t}\left(y^{\prime}\right) \varphi_{j}^{t}\left(0, z^{\prime}\right), \quad \hat{\xi}^{t}\left(y^{\prime}, z^{\prime}\right)=\hat{\psi}^{t}\left(y^{\prime}\right) \hat{\varphi}^{t}\left(z^{\prime}\right) .
$$

By virtue of (5.4), we have

$$
\operatorname{supp} \xi_{j}^{t}\left(y^{\prime}, z^{\prime}\right) \subset B_{\chi_{m} \delta\left(g^{t}\right)}, \quad \operatorname{supp} \hat{\xi}^{t}\left(y^{\prime}, z^{\prime}\right) \subset B_{\chi_{m} \delta\left(g^{t}\right)} .
$$

It is also clear that

$$
\begin{gathered}
\xi_{j}^{t}\left(y^{\prime}, z^{\prime}\right)=\varphi_{j}^{t}\left(0, z^{\prime}\right), \quad \hat{\xi}^{t}\left(y^{\prime}, z^{\prime}\right)=\hat{\varphi}^{t}\left(z^{\prime}\right), \quad\left|y^{\prime}\right|<\varepsilon_{1}^{\prime}, \\
\hat{\xi}^{t}\left(y^{\prime}, z^{\prime}\right) \xi_{j}^{t}\left(y^{\prime}, z^{\prime}\right)=\xi_{j}^{t}\left(y^{\prime}, z^{\prime}\right), \quad\left(y^{\prime}, z^{\prime}\right) \in \mathbb{R}^{n} .
\end{gathered}
$$

Denote by $\xi_{j}^{t}(x)$ and $\hat{\xi}_{j}^{t}(x)$ the functions $\xi_{j}^{t}\left(y^{\prime}, z^{\prime}\right)$ and $\hat{\xi}^{t}\left(y^{\prime}, z^{\prime}\right)$, respectively, written in the variables $x=x\left(g^{t}, j\right)$. Since $\operatorname{supp} \xi_{j}^{t}(x) \subset \hat{B}_{\delta\left(g^{t}\right)}\left(g_{j}^{t}\right)$ and $\operatorname{supp} \hat{\xi}_{j}^{t}(x) \subset \hat{B}_{\delta\left(g^{t}\right)}\left(g_{j}^{t}\right)$, we can extend these functions by zero outside the neighborhood $\hat{B}_{\delta\left(g^{t}\right)}\left(g_{j}^{t}\right)$ to obtain the functions infinitely differentiable on $\mathbb{R}^{n}$. 
Obviously, we have

$$
\sum_{j, t} \xi_{j}^{t}(x)=\sum_{j, t} \varphi_{j}^{t}(x)=1, \quad x \in \mathcal{K}_{1}
$$

2. We set

$$
\begin{aligned}
M_{H}^{t} & =\left\{u \in H_{a}^{l+2 m}(Q): \operatorname{supp} u \subset \bigcup_{j=1}^{N\left(g^{t}\right)} V\left(g_{j}^{t}\right)\right\}, \\
\mathcal{M}_{H}^{t} & =\left\{v \in \mathcal{H}_{a}^{l+2 m}(\Theta): \operatorname{supp} v \subset V(0)\right\} .
\end{aligned}
$$

For all $u \in M_{H}^{t}$, denote $u_{j}^{t}(x)=u(x), x \in V\left(g_{j}^{t}\right)$. We define the isomorphism $U^{t}: M_{H}^{t} \rightarrow$ $\mathcal{M}_{H}^{t}$ by the formulas

$$
\left(U^{t} u\right)_{j}\left(x^{\prime}\right)=u_{j}^{t}\left(x\left(x^{\prime}\right)\right), x^{\prime} \in \Theta_{j} \cap V(0) ; \quad\left(U^{t} u\right)_{j}\left(x^{\prime}\right)=0, x^{\prime} \in \Theta_{j} \backslash V(0) ;
$$

$j=1, \ldots, N\left(g^{t}\right)$.

We set

$$
\begin{aligned}
& G_{H}^{t}=\left\{f \in \mathcal{H}_{a}^{l}(Q, \Gamma): \operatorname{supp} f \subset \bigcup_{j=1}^{N\left(g^{t}\right)} V\left(g_{j}^{t}\right)\right\}, \\
& \mathcal{G}_{H}^{t}=\left\{\Phi \in \mathcal{H}_{a}^{l}(\Theta, \Gamma): \operatorname{supp} \Phi \subset V(0)\right\} .
\end{aligned}
$$

If $f=\left\{f_{0}, f_{i \mu}\right\} \in G_{H}^{t}$, then we denote by $f_{j}^{t}(x)$ and $f_{j \rho \mu}^{t}(x)$ the functions $f_{0}(x)$ and $f_{i \mu}(x)$ for $x \in V\left(g_{j}^{t}\right)$ and $x \in \Gamma_{i} \cap V\left(g_{j}^{t}\right)$, respectively, where $\rho$ and $j$ are such that the transformation $x \mapsto x^{\prime}\left(g^{t}, j\right)$ maps $\Gamma_{i} \cap V\left(g_{j}^{t}\right)$ onto $\Gamma_{j \rho} \cap V(0)$. Further, we define the isomorphism $F^{t}: G_{H}^{t} \rightarrow \mathcal{G}_{H}^{t}$ by the formula

$$
\left(F^{t} f\right)\left(x^{\prime}\right)=\left\{\left(F^{t} f\right)_{j}\left(x^{\prime}\right),\left(F^{t} f\right)_{j \rho \mu}\left(x^{\prime}\right)\right\} .
$$

Here

$$
\begin{gathered}
\left(F^{t} f\right)_{j}\left(x^{\prime}\right)=f_{j}^{t}\left(x\left(x^{\prime}\right)\right), x^{\prime} \in \Theta_{j} \cap V(0) ; \quad\left(F^{t} f\right)_{j}\left(x^{\prime}\right)=0, x^{\prime} \in \Theta_{j} \backslash V(0) ; \\
\left(F^{t} f\right)_{j \rho \mu}\left(x^{\prime}\right)=f_{j \rho \mu}^{t}\left(x\left(x^{\prime}\right)\right), x^{\prime} \in \Gamma_{j \rho} \cap V(0) ; \quad\left(F^{t} f\right)_{j \rho \mu}\left(x^{\prime}\right)=0, x^{\prime} \in \Gamma_{j \rho} \backslash V(0) ;
\end{gathered}
$$

$j=1, \ldots, N\left(g^{t}\right) ; \rho=1,2 ; \mu=1, \ldots, m$.

3. We set

$$
R_{\mathcal{K}_{1}} f=\sum_{t}\left(U^{t}\right)^{-1}\left(\hat{\xi}^{t}\left(\mathcal{L}_{g^{t}}^{\prime \prime}\right)^{-1} F^{t}\left(\sum_{q} \xi_{q}^{t} f\right)\right)
$$

Since the functions $\hat{\xi}^{t}$ and $\xi_{q}^{t}$ do not depend on $\varepsilon$, it follows from Corollary 2.1 that

$$
\left\|R_{\mathcal{K}_{1}} f\right\|_{H_{a}^{l+2 m}(Q)} \leq c_{1}\|f\|_{\mathcal{H}_{a}^{l}(Q, \Gamma)}
$$

where $c_{1}, c_{2}, \ldots>0$ depend neither on $\varepsilon$ nor on a function occurring on the right-hand side.

Clearly, we have

$$
\mathbf{L}^{1} R_{\mathcal{K}_{1}} f=\mathbf{L} R_{\mathcal{K}_{1}} f+T_{1} f
$$

where $T_{1}: \mathcal{H}_{a}^{l}(Q, \Gamma) \rightarrow \mathcal{H}_{a}^{l}(Q, \Gamma)$ is a linear bounded operator given by

$$
T_{1} f=\left\{0,-\left.\sum_{s=1}^{S_{i}}\left(B_{i \mu s}^{0}(x, D)\left((1-\xi) R_{\mathcal{K}_{1}} f\right)\right)\left(\omega_{i s}(x)\right)\right|_{\Gamma_{i}}\right\}
$$

(recall that $A^{1}=0$ and $B^{3}=0$ ). Moreover, using Lemmas 4.6 and 4.2 and estimate (5.10), we have

$$
\left\|T_{1} f\right\|_{\mathcal{H}_{a}^{l}(Q, \Gamma)} \leq c_{2}\left(\left\|R_{\mathcal{K}_{1}} f\right\|_{H_{a}^{l+2 m}(Q)}+\left\|\xi R_{\mathcal{K}_{1}} f\right\|_{H_{a}^{l+2 m}(Q)}\right) \leq c_{3}\|f\|_{\mathcal{H}_{a}^{l}(Q, \Gamma)} .
$$


Further, by virtue of (5.5), we have

$$
\operatorname{supp} \hat{\xi}^{t}\left(y^{\prime}, z^{\prime}\right) \subset B_{\delta}\left(g^{t}\right) .
$$

Therefore,

$$
\hat{\xi}^{t} \mathcal{L}_{g^{t}}^{\prime} v=\hat{\xi}^{t} \mathcal{L}_{g^{t}}^{\prime \prime} v, \quad v \in \mathcal{H}_{a}^{l+2 m}(\Theta) .
$$

It follows from Leibniz' formula that

$$
\mathcal{L}_{g^{t}}^{\prime}\left(\hat{\xi}^{t} v\right)=\hat{\xi}^{t} \mathcal{L}_{g^{t}}^{\prime} v+\tilde{\mathcal{L}}_{g^{t}} v+\left\{0, \mathcal{T}_{j \rho \mu}^{t} v\right\}
$$

where $\tilde{\mathcal{L}}_{g^{t}}: \mathcal{H}_{a}^{l+2 m-1}(\Theta) \rightarrow \mathcal{H}_{a}^{l}(\Theta, \Gamma)$ is a bounded operator such that $\operatorname{supp} \tilde{\mathcal{L}}_{g^{t}} v \subset$ $B_{\delta\left(g^{t}\right)}$, while

$$
\mathcal{T}_{j \rho \mu}^{t} v=\left.\sum_{(k, s) \neq(j, 0)}\left(\hat{\xi}^{t}\left(\mathcal{G}_{j \rho k s} y^{\prime}, z^{\prime}\right)-\hat{\xi}^{t}\left(y^{\prime}, z^{\prime}\right)\right)\left(B_{j \rho \mu k s}^{0}\left(x^{\prime}, D\right) v_{k}\right)\left(\mathcal{G}_{j \rho k s} y^{\prime}, z^{\prime}\right)\right|_{\Gamma_{j \rho}}
$$

and $\operatorname{supp} \mathcal{T}_{j \rho \mu}^{t} v \subset B_{\delta\left(g^{t}\right)}$. Clearly,

$$
\mathcal{T}_{j \rho \mu}^{t}: \mathcal{H}_{a}^{l+2 m}(\Theta) \rightarrow H_{a}^{l+2 m-m_{j \rho \mu}-1 / 2}\left(\Gamma_{j \rho}\right)
$$

is a bounded operator. Moreover, since the function $\hat{\xi}^{t}$ does not depend on $\varepsilon$, it follows that

$$
\begin{gathered}
\left\|\tilde{\mathcal{L}}_{g^{t}} v\right\|_{\mathcal{H}_{a}^{l}(\Theta, \Gamma)} \leq c_{4}\|v\|_{\mathcal{H}_{a}^{l+2 m-1}(\Theta)}, \\
\left\|\mathcal{T}_{j \rho \mu}^{t} v\right\|_{H_{a}^{l+2 m-m_{j \rho \mu}-1 / 2}\left(\Gamma_{j \rho}\right)} \leq c_{5}\|v\|_{\mathcal{H}_{a}^{l+2 m}(\Theta)}
\end{gathered}
$$

Using definition (5.9) of the operator $R_{\mathcal{K}_{1}}$, the isomorphisms $U^{t}$ and $F^{t}$, and relations (5.14), (5.13), and (5.7), we obtain

$$
\begin{aligned}
\mathbf{L} R_{\mathcal{K}_{1}} f & =\sum_{t}\left(F^{t}\right)^{-1} \mathcal{L}_{g^{t}}^{\prime} \hat{\xi}^{t}\left(\mathcal{L}_{g^{t}}^{\prime \prime}\right)^{-1} F^{t}\left(\sum_{q} \xi_{q}^{t} f\right) \\
& =\sum_{t}\left(F^{t}\right)^{-1} \hat{\xi}^{t} F^{t}\left(\sum_{q} \xi_{q}^{t} f\right)+T_{2} f+T_{3} f \\
& =\sum_{t}\left(F^{t}\right)^{-1} F^{t}\left(\sum_{q} \hat{\xi}_{q}^{t} \xi_{q}^{t} f\right)+T_{2} f+T_{3} f \\
& =\sum_{t, q} \xi_{q}^{t} f+T_{2} f+T_{3} f,
\end{aligned}
$$

where

$$
\begin{gathered}
T_{2} f=\sum_{t}\left(F^{t}\right)^{-1} \tilde{\mathcal{L}}_{g^{t}}\left(\mathcal{L}_{g^{t}}^{\prime \prime}\right)^{-1} F^{t}\left(\sum_{q} \xi_{q}^{t} f\right), \\
T_{3} f=\sum_{t}\left(F^{t}\right)^{-1}\left\{0, \mathcal{T}_{j \rho \mu}^{t}\left(\mathcal{L}_{g^{t}}^{\prime \prime}\right)^{-1} F^{t}\left(\sum_{q} \xi_{q}^{t} f\right)\right\} .
\end{gathered}
$$

Since the operator $\tilde{\mathcal{L}}_{g^{t}}: \mathcal{H}_{a}^{l+2 m-1}(\Theta) \rightarrow \mathcal{H}_{a}^{l}(\Theta, \Gamma)$ is bounded, supp $\tilde{\mathcal{L}}_{g^{t}} v \subset B_{\delta\left(g^{t}\right)}$, and $\mathcal{H}_{a}^{l+2 m}\left(\Theta \cap B_{\delta\left(g^{t}\right)}\right)$ is compactly embedded into $\mathcal{H}_{a}^{l+2 m-1}\left(\Theta \cap B_{\delta\left(g^{t}\right)}\right)$, it follows that the operator

$$
T_{2}: \mathcal{H}_{a}^{l}(Q, \Gamma) \rightarrow \mathcal{H}_{a}^{l}(Q, \Gamma)
$$

is compact. Furthermore, by (5.15), (5.16), and Corollary 2.1, we have

$$
\left\|T_{i} f\right\|_{\mathcal{H}_{a}^{l}(Q, \Gamma)} \leq c_{6}\|f\|_{\mathcal{H}_{a}^{l}(Q, \Gamma)}, \quad i=2,3
$$

(we have also used the fact that the operator $\mathcal{L}_{g^{t}}^{\prime \prime}$ and the functions $\xi_{q}^{t}$ do not depend on $\varepsilon)$. 
Now let us prove that the square of the operator $T_{3}$ is compact. Indeed,

$$
\left\|\left(T_{3}\right)^{2} f\right\|_{\mathcal{H}_{a}^{l}(Q, \Gamma)} \leq c_{7} \sum_{t, j, \rho, \mu}\left\|\mathcal{T}_{j \rho \mu}^{t}\left(\mathcal{L}_{g^{t}}^{\prime \prime}\right)^{-1} F^{t}\left(\sum_{q} \xi_{q}^{t} T_{3} f\right)\right\|_{H_{a}^{l+2 m-m_{j \rho \mu}-1 / 2}\left(\Gamma_{j \rho}\right)} .
$$

It follows from (5.5) and (5.6) that

$$
\operatorname{supp}\left(\hat{\xi}^{t}\left(\mathcal{G}_{j \rho k s} y^{\prime}, z^{\prime}\right)-\hat{\xi}^{t}\left(y^{\prime}, z^{\prime}\right)\right) \subset B_{\delta\left(g^{t}\right)}
$$

and

$$
\hat{\xi}^{t}\left(\mathcal{G}_{j \rho k s} y^{\prime}, z^{\prime}\right)-\hat{\xi}^{t}\left(y^{\prime}, z^{\prime}\right)=0
$$

for $\left|y^{\prime}\right| \leq \varepsilon_{1}^{\prime} / \chi_{M}$, where $\chi_{M}=\max _{j, \rho, k, s} \chi_{j \rho k s}$. Therefore, the condition $d_{k 1}<d_{j \rho}+\varphi_{j \rho k s}<$ $d_{k 2}$ for $(k, s) \neq(j, 0)$ implies that

$$
\left\|\mathcal{T}_{j \rho \mu}^{t} v\right\|_{H_{a}^{l+2 m-m_{j \rho \mu}-1 / 2}\left(\Gamma_{j \rho}\right)} \leq c_{8} \sum_{k=1}^{N\left(g^{t}\right)}\left\|v_{k}\right\|_{H_{a}^{l+2 m}\left(\Omega_{k}^{t}\right)},
$$

where

$$
\Omega_{k}^{t}=\left\{x^{\prime}=\left(y^{\prime}, z^{\prime}\right): d_{k 1}+d_{0}<\varphi<d_{k 2}-d_{0},\left|y^{\prime}\right|>\varepsilon_{1}^{\prime} / \chi_{M},\left|x^{\prime}\right|<\delta\left(g^{t}\right)\right\}
$$

and

$$
d_{0}=\min _{j, \rho, k, s}\left(d_{j \rho}+\varphi_{j \rho k s}-d_{k 1}, d_{k 2}-\left(d_{j \rho}+\varphi_{j \rho k s}\right)\right) / 2 \quad((k, s) \neq(j, 0)) .
$$

Using inequality (5.20), Lemma 3.5, and the equivalence of the norms in subspaces of the spaces $H_{a}^{l}\left(\Theta_{k}\right)$ and $W^{l}\left(\Theta_{k}\right)$ consisting of compactly supported functions vanishing near the edge $\mathcal{P}$, we obtain

$$
\begin{aligned}
& \left\|\mathcal{T}_{j \rho \mu}^{t}\left(\mathcal{L}_{g^{t}}^{\prime \prime}\right)^{-1} F^{t}\left(\sum_{q} \xi_{q}^{t} T_{3} f\right)\right\|_{H_{a}^{l+2 m-m_{j \rho \mu}-1 / 2}\left(\Gamma_{j \rho}\right)} \\
& \leq c_{9} \sum_{k}\left(\left\|A_{k}^{\prime \prime}\left[\left(\mathcal{L}_{g^{t}}^{\prime \prime}\right)^{-1} F^{t}\left(\sum_{q} \xi_{q}^{t} T_{3} f\right)\right]_{k}\right\|_{H_{a}^{l}\left(\Theta_{k}\right)}\right. \\
& \left.\quad+\left\|\left[\left(\mathcal{L}_{g^{t}}^{\prime \prime}\right)^{-1} F^{t}\left(\sum_{q} \xi_{q}^{t} T_{3} f\right)\right]_{k}\right\|_{H_{a}^{0}\left(\Theta_{k} \cap B_{2 \delta\left(g^{t}\right)}\right)}\right),
\end{aligned}
$$

where

$$
A_{k}^{\prime \prime}=A_{k}\left(D_{x^{\prime}}\right)+\eta\left(A_{k}^{0}\left(x^{\prime}, D_{x^{\prime}}\right)-A_{k}\left(D_{x^{\prime}}\right)\right) .
$$

However, the first $N\left(g^{t}\right)$ components of the vector $F^{t}\left(\sum_{q} \xi_{q}^{t} T_{3} f\right)$ are equal to zero. Therefore,

$$
A_{k}^{\prime \prime}\left[\left(\mathcal{L}_{g^{t}}^{\prime \prime}\right)^{-1} F^{t}\left(\sum_{q} \xi_{q}^{t} T_{3} f\right)\right]_{k}=0, \quad k=1, \ldots, N\left(g^{t}\right)
$$

and hence

$$
\begin{aligned}
& \left\|\mathcal{T}_{j \rho \mu}^{t}\left(\mathcal{L}_{g^{t}}^{\prime \prime}\right)^{-1} F^{t}\left(\sum_{q} \xi_{q}^{t} T_{3} f\right)\right\|_{H_{a}^{l+2 m-m_{j \rho \mu}-1 / 2}\left(\Gamma_{j \rho}\right)} \\
& \quad \leq c_{9} \sum_{k}\left\|\left[\left(\mathcal{L}_{g^{t}}^{\prime \prime}\right)^{-1} F^{t}\left(\sum_{q} \xi_{q}^{t} T_{3} f\right)\right]_{k}\right\|_{H_{a}^{0}\left(\Theta_{k} \cap B_{2 \delta\left(g^{t}\right)}\right)} .
\end{aligned}
$$

Inequalities (5.19) and (5.23) and the compactness of the embedding $H_{a}^{l+2 m}\left(\Theta_{k}\right) \subset$ $H_{a}^{0}\left(\Theta_{k} \cap B_{2 \delta\left(g^{t}\right)}\right)$ imply that the operator $T_{3}$ has a compact square. 
Similarly, one can show that the operator $T_{1}$ has a compact square. To this end, one must use the relation

$$
\left[\overline{\omega_{i s}\left(\Gamma_{i}\right)} \cap\left(\bigcup_{t, j} \hat{B}_{\delta\left(g_{j}^{t}\right)}\left(g_{j}^{t}\right)\right)\right] \backslash \mathcal{K}_{1}^{\varepsilon / 2} \subset Q_{b}, \quad i=1, \ldots, N_{0}, s=1, \ldots, S_{i},
$$

which holds for some $b>0$.

Thus, it follows from (5.11) and (5.17) that

$$
\mathbf{L}^{1} R_{\mathcal{K}_{1}} f=\xi_{0} f+T_{\mathcal{K}_{1}} f,
$$

where

$$
\xi_{0}(x)=\sum_{t, q} \xi_{q}^{t}(x)
$$

and $T_{\mathcal{K}_{1}}: \mathcal{H}_{a}^{l}(Q, \Gamma) \rightarrow \mathcal{H}_{a}^{l}(Q, \Gamma)$ is a bounded operator with compact square. Moreover, inequalities (5.12) and (5.18) imply that

$$
\left\|T_{\mathcal{K}_{1}} f\right\|_{\mathcal{H}_{a}^{l}(Q, \Gamma)} \leq c_{10}\|f\|_{\mathcal{H}_{a}^{l}(Q, \Gamma)} .
$$

4. Take a number $\varepsilon$ in the definition of the function $\xi$ so small that

$$
\mathcal{K}_{1}^{4 \varepsilon} \subset \bigcup_{t, j} \hat{B}_{\delta\left(g^{t}\right)}\left(g_{j}^{t}\right)
$$

and

$$
\xi_{0}(x) \geq 1 / 2, \quad x \in \mathcal{K}_{1}^{4 \varepsilon}
$$

(the existence of such an $\varepsilon$ follows from (5.8) and (5.25)). Later on, we will impose some additional conditions on $\varepsilon$.

For each $\varepsilon$, we consider a function $\zeta_{0} \in C_{0}^{\infty}\left(\mathbb{R}^{n}\right)$ depending on $\varepsilon$, such that

$$
\operatorname{supp} \zeta_{0} \subset \mathcal{K}_{1}^{4 \varepsilon} ; \quad \zeta_{0}(x)=1, x \in \mathcal{K}_{1}^{2 \varepsilon} ; \quad\left|D^{\alpha} \zeta_{0}(x)\right| \leq c_{11} \varepsilon^{-|\alpha|} .
$$

For each point $g \in \bar{Q} \backslash \mathcal{K}_{1}^{2 \varepsilon}$, we consider its $(\varepsilon / 2)$-neighborhood $B_{\varepsilon / 2}(g)$. All these neighborhoods cover $\bar{Q} \backslash \mathcal{K}_{1}^{2 \varepsilon}$. Choose a finite subcovering $\left\{B_{\varepsilon / 2}\left(h^{\tau}\right)\right\}_{\tau=1}^{\tau_{1}}$, where $\tau_{1}=$ $\tau_{1}(\varepsilon)$. Let functions $\tilde{\zeta}^{\tau} \in C_{0}^{\infty}\left(\mathbb{R}^{n}\right)$ form a partition of unity for $\bar{Q} \backslash \mathcal{K}_{1}^{2 \varepsilon}$, subordinate to the covering $\left\{B_{\varepsilon / 2}\left(h^{\tau}\right)\right\}_{\tau=1}^{\tau_{1}}$. Then the functions

$$
\zeta=\xi_{0}+\zeta_{0}\left(1-\xi_{0}\right), \quad \zeta^{\tau}=(1-\zeta) \tilde{\zeta}^{\tau}, \quad \tau=1, \ldots, \tau_{1},
$$

form a partition of unity for $\bar{Q}$, subordinate to the covering by the sets $\bigcup_{t, j} \hat{B}_{\delta\left(g^{t}\right)}\left(g_{j}^{t}\right)$ and $B_{\varepsilon / 2}\left(h^{\tau}\right), \tau=1, \ldots, \tau_{1}$.

Due to Theorem 2.3 and to the general theory of elliptic boundary-value problems in the interior of a domain and near a smooth part of the boundary (see, e.g., 31]), there exist bounded operators

$$
R_{\tau 0}:\left\{f \in \mathcal{H}_{a}^{l}(Q, \Gamma): \operatorname{supp} f \subset B_{\varepsilon / 2}\left(h^{\tau}\right)\right\} \rightarrow\left\{u \in H_{a}^{l+2 m}(Q): \operatorname{supp} u \subset B_{\varepsilon}\left(h^{\tau}\right)\right\}
$$

and compact operators

$$
T_{\tau 0}:\left\{f \in \mathcal{H}_{a}^{l}(Q, \Gamma): \operatorname{supp} f \subset B_{\varepsilon / 2}\left(h^{\tau}\right)\right\} \rightarrow\left\{f \in \mathcal{H}_{a}^{l}(Q, \Gamma): \operatorname{supp} f \subset B_{\varepsilon}\left(h^{\tau}\right)\right\}
$$

such that

$$
\mathbf{L}^{0} R_{\tau 0} f=f+T_{\tau 0} f .
$$

For any $f \in \mathcal{H}_{a}^{l}(Q, \Gamma)$, we set

$$
R f=R_{\mathcal{K}_{1}} f+R_{\mathcal{K}_{1}}(\eta f)+\sum_{\tau} R_{\tau 0}\left(\zeta^{\tau} f\right),
$$


where $\eta(x)=\zeta_{0}(x)\left(1-\xi_{0}(x)\right) / \xi_{0}(x)$ for $x \in \mathcal{K}_{1}^{4 \varepsilon}$ and $\eta(x)=0$ for $x \notin \mathcal{K}_{1}^{4 \varepsilon}$. Note that $\operatorname{supp} \zeta_{0} \subset \mathcal{K}_{1}^{4 \varepsilon}$ and $\xi_{0}(x) \geq 1 / 2$ for $x \in \mathcal{K}_{1}^{4 \varepsilon}$; hence, the function $\eta$ is supported on $\mathcal{K}_{1}^{4 \varepsilon}$ and infinitely differentiable on $\mathbb{R}^{n}$. We have

$$
\mathbf{L}^{1} R f=\mathbf{L}^{1} R_{\mathcal{K}_{1}} f+\mathbf{L}^{1} R_{\mathcal{K}_{1}}(\eta f)+\sum_{\tau} \mathbf{L}^{0} R_{\tau 0}\left(\zeta^{\tau} f\right)+\sum_{\tau}\left\{0, B_{i \mu}^{1} R_{\tau 0}\left(\zeta^{\tau} f\right)\right\} .
$$

Since $\zeta(x)=1$ for $x \in \mathcal{K}_{1}^{2 \varepsilon}$, it follows that $\operatorname{supp} \zeta^{\tau} f \subset \bar{Q} \backslash \mathcal{K}_{1}^{2 \varepsilon}$. Thus, we see that $\operatorname{supp} R_{\tau 0}\left(\zeta^{\tau} f\right) \subset \bar{Q} \backslash \mathcal{K}_{1}^{\varepsilon}$, while $\operatorname{supp} \xi \subset \mathcal{K}_{1}^{\varepsilon}$. Therefore,

$$
B_{i \mu}^{1} R_{\tau 0}\left(\zeta^{\tau} f\right)=0
$$

Combining this relation with equalities (5.24), (5.29), and (5.31), we obtain

$$
\mathbf{L}^{1} R f=\xi_{0} f+T_{\mathcal{K}_{1}} f+\zeta_{0}\left(1-\xi_{0}\right) f+T_{\mathcal{K}_{1}}(\eta f)+\sum_{\tau} \zeta^{\tau} f+T f=f+T_{\mathcal{K}_{1}} f+M f+T f,
$$

where

$$
M f=T_{\mathcal{K}_{1}}(\eta f)
$$

while $T: \mathcal{H}_{a}^{l}(Q, \Gamma) \rightarrow \mathcal{H}_{a}^{l}(Q, \Gamma)$ is a compact operator (whose norm may increase as $\varepsilon \rightarrow 0)$. By (5.26), we have

$$
\|M f\|_{\mathcal{H}_{a}^{l}(Q, \Gamma)} \leq c_{10}\|\eta f\|_{\mathcal{H}_{a}^{l}(Q, \Gamma)} .
$$

However, $\left(1-\xi_{0}(x)\right) / \xi_{0}(x)=0$ for $x \in \mathcal{K}_{1}$ and the function $\zeta_{0}$ is supported in $\mathcal{K}_{1}^{4 \varepsilon}$ and satisfies the inequality in (5.27). Therefore, it follows from the last estimate, from Lemmas 4.1 and 4.2 and from Remark 4.1 that

$$
\|M f\|_{\mathcal{H}_{a}^{l}(Q, \Gamma)} \leq c_{11} \varepsilon\|f\|_{\mathcal{H}_{a}^{l}(Q, \Gamma)} .
$$

It follows from (5.26), (5.33), and from Lemma 8.1 that the operator $\mathbf{I}+T_{\mathcal{K}_{1}}+M$ has the Fredholm property, provided that $\varepsilon>0$ is sufficiently small. Applying Theorem 16.4 in [18, we see that the operator

$$
\mathbf{L}^{1} R=\mathbf{I}+T_{\mathcal{K}_{1}}+M+T
$$

also has the Fredholm property. Therefore, by Theorem 15.2 in 18 , there exist a bounded operator $R^{\prime}: \mathcal{H}_{a}^{l}(Q, \Gamma) \rightarrow \mathcal{H}_{a}^{l}(Q, \Gamma)$ and a compact operator $\mathbf{T}_{1}: \mathcal{H}_{a}^{l}(Q, \Gamma) \rightarrow \mathcal{H}_{a}^{l}(Q, \Gamma)$ such that

$$
\mathbf{L}^{1} R R^{\prime}=\mathbf{I}+\mathbf{T}_{1} .
$$

Setting $\mathbf{R}_{1}=R R^{\prime}$, we complete the proof.

Set $\mathcal{H}_{a}^{l}(\partial Q)=\prod_{i, \mu} H_{a}^{l+2 m-m_{i \mu}-1 / 2}\left(\Gamma_{i}\right)$.

To construct a right regularizer for the operator $\mathbf{L}$, we also need to prove the existence of a "right regularizer" $\mathbf{R}_{1}^{\prime}$ for the operator $\mathbf{L}^{1}$, which is defined on the functions $f^{\prime} \in$ $\mathcal{H}_{a}^{l}(\partial Q)$ and possesses the following properties: $\mathbf{R}_{1}^{\prime} f^{\prime}$ is supported near the boundary $\partial Q$ for all $f^{\prime}$ and $\mathbf{R}_{1}^{\prime} f^{\prime}$ is supported near the set $\mathcal{K}_{1}$ for $f^{\prime}$ supported near $\mathcal{K}_{1}$.

Lemma 5.3. Let the conditions of Theorem 5.1 be fulfilled. Then there exist a linear bounded operator $\mathbf{R}_{1}^{\prime}: \mathcal{H}_{a}^{l}(\partial Q) \rightarrow H_{a}^{l+2 m}(Q)$ and a compact operator $\mathbf{T}_{1}^{\prime}: \mathcal{H}_{a}^{l}(\partial Q) \rightarrow$ $\mathcal{H}_{a}^{l}(Q, \Gamma)$ such that

$$
\begin{gathered}
\mathbf{L}^{1} \mathbf{R}_{1}^{\prime} f^{\prime}=\left\{0, f^{\prime}\right\}+\mathbf{T}_{1}^{\prime} f^{\prime} \\
\quad \operatorname{supp} \mathbf{R}_{1}^{\prime} f^{\prime} \subset \bar{Q} \backslash Q_{\sigma}
\end{gathered}
$$

for any $f^{\prime} \in \mathcal{H}_{a}^{l}(\partial Q)$, and

$$
\operatorname{supp} \mathbf{R}_{1}^{\prime} f^{\prime} \subset \mathcal{K}_{1}^{2 \varkappa}
$$

for $f^{\prime} \in \mathcal{H}_{a}^{l}(\partial Q), \operatorname{supp} f^{\prime} \subset \overline{\mathcal{K}_{1}^{\varkappa}}$, where $\varkappa, \sigma>0$ are the constants from Lemma 4.6 , 
Proof. 1. Fix an arbitrary number $\hat{\varepsilon}>0$ independent of $\varepsilon$. Similarly to the proof of Lemma 5.2 we can construct functions $\hat{\zeta}, \hat{\zeta}^{\tau} \in C_{0}^{\infty}\left(\mathbb{R}^{n}\right), \tau=1, \ldots, \hat{\tau}_{1}, \hat{\tau}_{1}=\hat{\tau}_{1}(\hat{\varepsilon})$, which form a partition of unity for $\bar{Q}$, subordinate to the covering by the sets $\mathcal{K}_{1}^{2 \hat{\varepsilon}}$ and $B_{\hat{\varepsilon} / 2}\left(h^{\tau}\right)$, $\tau=1, \ldots, \hat{\tau}_{1}$, where $h^{\tau} \in \bar{Q} \backslash \mathcal{K}_{1}^{2 \hat{\varepsilon}}$. In particular, the function $\hat{\zeta}$ can be chosen in such a way that

$$
\hat{\zeta}(x)=1, \quad x \in \mathcal{K}_{1}^{\hat{\varepsilon}} .
$$

2. Due to Theorem 2.3 and to the general theory of elliptic boundary-value problems near a smooth part of the boundary (see, e.g., 31]), there exist bounded operators

$$
R_{\tau 0}^{\prime}:\left\{f^{\prime} \in \mathcal{H}_{a}^{l}(\partial Q): \operatorname{supp} f^{\prime} \subset B_{\hat{\varepsilon} / 2}\left(h^{\tau}\right)\right\} \rightarrow\left\{u \in H_{a}^{l+2 m}(Q): \operatorname{supp} u \subset B_{\hat{\varepsilon}}\left(h^{\tau}\right)\right\}
$$

and compact operators

$$
T_{\tau 0}^{\prime}:\left\{f^{\prime} \in \mathcal{H}_{a}^{l}(\partial Q): \operatorname{supp} f^{\prime} \subset B_{\hat{\varepsilon} / 2}\left(h^{\tau}\right)\right\} \rightarrow\left\{f \in \mathcal{H}_{a}^{l}(Q, \Gamma): \operatorname{supp} f \subset B_{\hat{\varepsilon}}\left(h^{\tau}\right)\right\}
$$

such that

$$
\mathbf{L}^{0} R_{\tau 0}^{\prime} f^{\prime}=\left\{0, f^{\prime}\right\}+T_{\tau 0}^{\prime} f^{\prime} .
$$

For any $f^{\prime} \in \mathcal{H}_{a}^{l}(\partial Q)$, we set

$$
\mathbf{R}_{1}^{\prime} f^{\prime}=\hat{\zeta} u+\sum_{\tau} u^{\tau}
$$

where

$$
u=\mathbf{R}_{1}\left\{0, f^{\prime}\right\}, \quad u^{\tau}=R_{\tau 0}^{\prime}\left(\hat{\zeta}^{\tau}\left\{0, f^{\prime}\right\}\right) .
$$

Clearly, property (5.35) holds for $2 \hat{\varepsilon}<\sigma$, while property (5.36) holds for $2 \hat{\varepsilon}<2 \varkappa$ and $\varkappa+\hat{\varepsilon} / 2<2 \varkappa$. Let us prove relation (5.34).

Using (5.39) and Leibniz' formula, we have

$$
\mathbf{L}^{1} \mathbf{R}_{1}^{\prime} f^{\prime}=\hat{\zeta} \mathbf{L}^{1} u+\left\{0, \sum_{s=1}^{S_{i}} T_{i \mu s} f^{\prime}\right\}+T u+\sum_{\tau} \mathbf{L}^{0} u^{\tau}+\left\{0, B_{i \mu}^{1} u^{\tau}\right\}
$$

where

$$
T_{i \mu s} f^{\prime}=\left.\left(\hat{\zeta}\left(\omega_{i s}(x)\right)-\hat{\zeta}(x)\right)\left(B_{i \mu s}^{0}\left(x, D_{x}\right)(\xi u)\right)\left(\omega_{i s}(x)\right)\right|_{\Gamma_{i}},
$$

while $T: H_{a}^{l+2 m-1}(Q) \rightarrow \mathcal{H}_{a}^{l}(Q, \Gamma)$ is a bounded operator. By virtue of the compactness of the embedding $H_{a}^{l+2 m}(Q) \subset H_{a}^{l+2 m-1}(Q)$, the operator $T: H_{a}^{l+2 m}(Q) \rightarrow \mathcal{H}_{a}^{l}(Q, \Gamma)$ is compact.

Now it follows from Lemma 5.2, from (5.38), and from (5.40) that

$$
\mathbf{L}^{1} \mathbf{R}_{1}^{\prime} f^{\prime}=\left\{0, f^{\prime}\right\}+T^{\prime} f^{\prime}+\left\{0, \sum_{s=1}^{S_{i}} T_{i \mu s} f^{\prime}\right\}+\left\{0, B_{i \mu}^{1} u^{\tau}\right\}
$$

where $T^{\prime}: \mathcal{H}_{a}^{l}(\partial Q) \rightarrow \mathcal{H}_{a}^{l}(Q, \Gamma)$ is a compact operator.

3. Let us prove that the operator $T_{i \mu s}: \mathcal{H}_{a}^{l}(\partial Q) \rightarrow H_{a}^{l+2 m-m_{i \mu}-1 / 2}\left(\Gamma_{i}\right)$ is compact. Since $\omega_{i s}$ are $C^{\infty}$ diffeomorphisms, it follows from Lemma 4.5 that

$$
\begin{aligned}
& \left\|T_{i \mu s} f^{\prime}\right\|_{H_{a}^{l+2 m-m_{i \mu}-1 / 2}\left(\Gamma_{i}\right)} \\
& \quad \leq k_{1}\left\|\left.\left(\hat{\zeta}(x)-\hat{\zeta}\left(\omega_{i s}^{-1}(x)\right)\right)\left(B_{i \mu s}^{0}\left(x, D_{x}\right)(\xi u)\right)\right|_{\omega_{i s}\left(\Gamma_{i}\right)}\right\|_{H_{a}^{l+2 m-m_{i \mu}-1 / 2}\left(\omega_{i s}\left(\Gamma_{i}\right)\right)},
\end{aligned}
$$

where $k_{1}, k_{2}, \ldots>0$ do not depend on $f^{\prime}$.

Denote $\mathcal{M}_{i s}=\overline{\omega_{i s}\left(\Gamma_{i}\right)} \backslash \omega_{i s}\left(\Gamma_{i}\right)$. For every $x \in \mathcal{M}_{i s}$, we have either $x \in \mathcal{K}_{1}$, or $x \in \mathcal{K}_{2}$, or $x \in \mathcal{K}_{3}$. If $x \in \mathcal{M}_{i s} \cap \mathcal{K}_{1}$, then both $x$ and $\omega_{i s}^{-1}(x)$ belong to $\mathcal{K}_{1}$. Therefore,

$$
\hat{\zeta}(x)-\hat{\zeta}\left(\omega_{i s}^{-1}(x)\right)=0, \quad x \in\left(\mathcal{M}_{i s} \cap \mathcal{K}_{1}\right)^{d},
$$


where $\left(\mathcal{M}_{i s} \cap \mathcal{K}_{1}\right)^{d}$ is the $d$-neighborhood of the set $\mathcal{M}_{i s} \cap \mathcal{K}_{1}$ and $d>0$ is sufficiently small.

If $\mathcal{M}_{i s} \cap\left(\mathcal{K}_{2} \cup \mathcal{K}_{3}\right) \neq \varnothing$, then

$$
\xi(x)=0, \quad x \in\left(\mathcal{M}_{i s} \cap\left(\mathcal{K}_{2} \cup \mathcal{K}_{3}\right)\right)^{d},
$$

where $\left(\mathcal{M}_{i s} \cap\left(\mathcal{K}_{2} \cup \mathcal{K}_{3}\right)\right)^{d}$ is the $d$-neighborhood of the set $\mathcal{M}_{i s} \cap\left(\mathcal{K}_{2} \cup \mathcal{K}_{3}\right)$ and $d>0$ is sufficiently small.

In all these cases, we see that

$$
\left.\operatorname{supp}\left(\hat{\zeta}(x)-\hat{\zeta}\left(\omega_{i s}^{-1}(x)\right)\right)\left(B_{i \mu s}^{0}\left(x, D_{x}\right)(\xi u)\right)\right|_{\omega_{i s}\left(\Gamma_{i}\right)} \subset Q_{b},
$$

where $b>0$ is sufficiently small.

Using estimate (5.43), Lemma 3.5. and the equivalence of the norms in $H_{a}^{l}\left(Q_{b}\right)$ and $W^{l}\left(Q_{b}\right)$, we obtain

$$
\left\|T_{i \mu s} f^{\prime}\right\|_{H_{a}^{l+2 m-m_{i \mu}-1 / 2}\left(\Gamma_{i}\right)} \leq k_{2}\|u\|_{W^{l+2 m}\left(Q_{b}\right)} \leq k_{3}\left(\left\|A^{0} u\right\|_{H_{a}^{l}(Q)}+\|u\|_{H_{a}^{0}(Q)}\right) .
$$

By Lemma 5.2, $A^{0} u=A^{0} \mathbf{R}^{1}\left\{0, f^{\prime}\right\}=\mathbf{T}_{11} f^{\prime}$, where $\mathbf{T}_{11}: \mathcal{H}_{a}^{l}(\partial Q) \rightarrow H_{a}^{l}(Q)$ is a compact operator. Hence, inequality (5.44) takes the form

$$
\left\|T_{i \mu s} f^{\prime}\right\|_{H_{a}^{l+2 m-m_{i \mu}-1 / 2}\left(\Gamma_{i}\right)} \leq k_{3}\left(\left\|\mathbf{T}_{11} f^{\prime}\right\|_{H_{a}^{l}(Q)}+\left\|\mathbf{R}^{1}\left\{0, f^{\prime}\right\}\right\|_{H_{a}^{0}(Q)}\right),
$$

which implies that $T_{i \mu s}$ is a compact operator (because $\mathbf{T}_{11}$ is compact and $H_{a}^{l+2 m}(Q)$ is compactly embedded into $\left.H_{a}^{0}(Q)\right)$.

4. The expression $B_{i \mu}^{1} u^{\tau}$ consists of the terms

$$
\left.\left(B_{i \mu s}^{0}\left(x, D_{x}\right)\left(\xi R_{\tau 0}^{\prime}\left(\hat{\zeta}^{\tau}\left\{0, f^{\prime}\right\}\right)\right)\right)\left(\omega_{i s}(x)\right)\right|_{\Gamma_{i}}, \quad s=1, \ldots, S_{i} .
$$

Since supp $R_{\tau 0}^{\prime}\left(\hat{\zeta}^{\tau}\left\{0, f^{\prime}\right\}\right) \subset B_{\hat{\varepsilon}}\left(h^{\tau}\right)$, where $h^{\tau} \in \bar{Q} \backslash \mathcal{K}_{1}^{2 \hat{\varepsilon}}$, it follows that

$$
\operatorname{supp} R_{\tau 0}^{\prime}\left(\hat{\zeta}^{\tau}\left\{0, f^{\prime}\right\}\right) \subset \bar{Q} \backslash \mathcal{K}_{1}^{\hat{\varepsilon}} .
$$

On the other hand, $\operatorname{supp} \xi \subset \mathcal{K}_{1}^{\varepsilon}$. Hence,

$$
\left.\operatorname{supp} B_{i \mu s}^{0}\left(x, D_{x}\right)\left(\xi R_{\tau 0}^{\prime}\left(\hat{\zeta}^{\tau}\left\{0, f^{\prime}\right\}\right)\right)\right|_{\omega_{i s}\left(\Gamma_{i}\right)} \subset Q_{b},
$$

where $b>0$ is sufficiently small. Therefore, applying Lemma 3.5] and equality (5.38), we can show similarly to the above that each of the operators in (5.45) is compact. Thus, we see that (5.42) is equivalent to (5.34).

Now we can prove Theorem 5.2

Proof of Theorem 5.2. 1. We set

$$
\Phi=B^{2} \mathbf{R}_{1} f, \quad f=\left\{f_{0}, f^{\prime}\right\} \in \mathcal{H}_{a}^{l}(Q, \Gamma) .
$$

Introduce the bounded operator $\mathbf{R}: \mathcal{H}_{a}^{l}(Q, \Gamma) \rightarrow H_{a}^{l+2 m}(Q)$ by the formula

$$
\mathbf{R} f=\mathbf{R}_{1} f-\mathbf{R}_{1}^{\prime} \Phi+\mathbf{R}_{1}^{\prime} B^{2} \mathbf{R}_{1}^{\prime} \Phi,
$$

where $\mathbf{R}_{1}$ and $\mathbf{R}_{1}^{\prime}$ are the operators occurring in Lemmas 5.2 and 5.3, respectively. Let us show that $\mathbf{R}$ is the desired operator.

For simplicity, we denote diverse compact operators by the same letter $T$.

It follows from Lemmas 5.2 and 5.3 that

$$
A \mathbf{R} f=A^{0} \mathbf{R} f=A^{0} \mathbf{R}_{1} f-A^{0} \mathbf{R}_{1}^{\prime}\left(\Phi-B^{2} \mathbf{R}_{1}^{\prime} \Phi\right)=f_{0}+T f
$$


(recall that $A^{1}=0$ ) and

$$
\begin{aligned}
C \mathbf{R} f & =C \mathbf{R}_{1} f-C \mathbf{R}_{1}^{\prime} \Phi+C \mathbf{R}_{1}^{\prime} B^{2} \mathbf{R}_{1}^{\prime} \Phi \\
& =\left(f^{\prime}+T f\right)-(\Phi+T \Phi)+\left(B^{2} \mathbf{R}_{1}^{\prime} \Phi+T \mathbf{B}^{2} \mathbf{R}_{1}^{\prime} \Phi\right) \\
& =f^{\prime}-\Phi+B^{2} \mathbf{R}_{1}^{\prime} \Phi+T f .
\end{aligned}
$$

Applying the operator $B^{2}$ to the function $\mathbf{R} f$ and using (5.46), we obtain

$$
B^{2} \mathbf{R} f=\Phi-B^{2} \mathbf{R}_{1}^{\prime} \Phi+B^{2} \mathbf{R}_{1}^{\prime} B^{2} \mathbf{R}_{1}^{\prime} \Phi .
$$

Summing relations (5.48) and (5.49) and recalling that $B^{3}=0$, we obtain

$$
B \mathbf{R} f=f^{\prime}+T f+B^{2} \mathbf{R}_{1}^{\prime} B^{2} \mathbf{R}_{1}^{\prime} \Phi .
$$

2. Let us show that

$$
B^{2} \mathbf{R}_{1}^{\prime} B^{2} \mathbf{R}_{1}^{\prime} \Phi=0 .
$$

It follows from relation (5.35) in Lemma 5.3 that

$$
\operatorname{supp} \mathbf{R}_{1}^{\prime} \Phi \subset \bar{Q} \backslash Q_{\sigma} .
$$

Therefore, estimate (4.25) implies that

$$
\operatorname{supp} B^{2} \mathbf{R}_{1}^{\prime} \Phi \subset \overline{\mathcal{K}_{1}^{\varkappa}} .
$$

Furthermore, it follows from relation (5.36) in Lemma 5.3 that

$$
\operatorname{supp} \mathbf{R}_{1}^{\prime} B^{2} \mathbf{R}_{1}^{\prime} \Phi \subset \mathcal{K}_{1}^{2 \varkappa} .
$$

Combining this fact with inequality (4.24) yields (5.51).

Relations (5.47), (5.50), and (5.51) prove the theorem.

Remark 5.1. Using the results in [7, one can show that Theorem 5.1] remains true for the case in which the transformations $\omega_{i s}$ are nonlinear near the set $\mathcal{K}_{1}$, while their linear parts at the points of the set $\mathcal{K}_{1}$ satisfy Condition 1.4. Moreover, the index of the problem with nonlinear transformations $\omega_{i s}$ is equal to the index of the corresponding problem with transformations linearized near the set $\mathcal{K}_{1}$.

\section{Some GeneralizAtions}

6.1. In this section, we generalize the results of Secs. 4 and 5 to the case where the diffeomorphisms $\omega_{i s}$ are defined only on some neighborhood of the set $\mathcal{K}_{1}$, the operators $B_{i \mu}^{2}$ are abstract nonlocal operators supported outside the set $\mathcal{K}_{1}$, and $A^{1}$ and $B_{i \mu}^{3}$ are compact perturbations on the corresponding weighted spaces.

Consider the differential operators

$$
A^{0} \equiv A^{0}(x, D)=\sum_{|\alpha|=2 m} a_{\alpha}(x) D^{\alpha}, \quad B_{i \mu s}^{0} \equiv B_{i \mu s}^{0}(x, D)=\sum_{|\alpha|=m_{i \mu}} b_{i \mu s \alpha}(x) D^{\alpha},
$$

where $a_{\alpha}, b_{i \mu s \alpha} \in C^{\infty}\left(\mathbb{R}^{n}\right)$ are complex-valued functions $\left(i=1, \ldots, N_{0} ; \mu=1, \ldots, m\right.$; $\left.s=0, \ldots, S_{i}\right), m_{i \mu} \leq 2 m-1$.

Let a domain $Q \subset \mathbb{R}^{n}$ satisfy the assumptions of Sec. 1 As in Sec. 1, we denote

$$
\mathcal{K}_{1}=\bigcup_{\nu=1}^{N_{1}} \mathcal{K}_{1 \nu}=\partial Q \backslash \bigcup_{i} \Gamma_{i},
$$

where $\mathcal{K}_{1 \nu}$ are mutually disjoint $(n-2)$-dimensional connected $C^{\infty}$ manifolds without a boundary.

Let $\omega_{i s}\left(i=1, \ldots, N_{0} ; s=1, \ldots, S_{i}\right)$ denote a $C^{\infty}$ diffeomorphism mapping $\left(\overline{\Gamma_{i}} \backslash \Gamma_{i}\right)^{\varepsilon_{0}}$ onto the set $\omega_{i s}\left(\left(\overline{\Gamma_{i}} \backslash \Gamma_{i}\right)^{\varepsilon_{0}}\right)$, where $\varepsilon_{0}>0$ is some number, in such a way that 
(1) $\omega_{i s}\left(\Gamma_{i} \cap \mathcal{K}_{1}^{\varepsilon_{0}}\right) \subset Q$,

(2) if $\eta\left(1 \leq \eta \leq N_{1}\right)$ and $i\left(1 \leq i \leq N_{0}\right)$ are such that $\mathcal{K}_{1 \eta} \subset \overline{\Gamma_{i}} \backslash \Gamma_{i}$, then, for every $s\left(1 \leq s \leq S_{i}\right)$, there is a $\nu\left(1 \leq \nu \leq N_{1}\right)$ such that $\omega_{i s}\left(\mathcal{K}_{1 \eta}\right)=\mathcal{K}_{1 \nu}$.

Along with the set $\mathcal{K}_{1}$, we introduce the set

$$
K_{2}=\bigcup_{\nu=1}^{N_{2}} K_{2 \nu} \subset \bigcup_{i} \Gamma_{i},
$$

where $K_{2 \nu}$ are mutually disjoint connected $C^{\infty}$ manifolds without a boundary. In particular, the set $K_{2}$ can be empty. We use either the set

$$
K=\mathcal{K}_{1}
$$

or the set

$$
K=\mathcal{K}_{1} \cup K_{2}
$$

in the definition of the space $H_{a}^{l}(Q)=H_{a}^{l}(Q, K)$.

We study the following nonlocal elliptic problem:

$$
\begin{gathered}
A u \equiv A^{0} u+A^{1} u=f_{0}(x), \quad x \in Q, \\
B_{i \mu} u \equiv \sum_{j=0}^{3} B_{i \mu}^{j} u=f_{i \mu}(x), \quad x \in \Gamma_{i} ; i=1, \ldots, N_{0} ; \mu=1, \ldots, m .
\end{gathered}
$$

Here

$$
B_{i \mu}^{0}=\left.B_{i \mu 0}^{0} u\right|_{\Gamma_{i}}, \quad B_{i \mu}^{1} u=\left.\sum_{s=1}^{S_{i}}\left(B_{i \mu s}^{0}(x, D)(\xi u)\right)\left(\omega_{i s}(x)\right)\right|_{\Gamma_{i}},
$$

a function $\xi \in C_{0}^{\infty}\left(\mathbb{R}^{n}\right)$ is such that $\xi(x)=1$ for $x \in \mathcal{K}_{1}^{\varepsilon / 2}$ and supp $\xi \subset K_{1}^{\varepsilon}$, while $\varepsilon>0$ is so small that if $\omega_{i s}\left(\mathcal{K}_{1 \eta}\right)=\mathcal{K}_{1 \nu}$, then $\mathcal{K}_{1 \nu}^{\varepsilon} \subset \omega_{i s}\left(\mathcal{K}_{1 \eta}^{\varepsilon_{0}}\right)$.

Assume that the operators $A^{0}$ and $B_{i \mu 0}^{0}$ satisfy Conditions 1.1 and 1.2 and that the transformations $\omega_{i s}$ satisfy Conditions 1.3 and 1.4 We also suppose that the following conditions for the operators $A^{1}, B_{i \mu}^{2}$, and $B_{i \mu}^{3}$ hold.

Condition 6.1 (Compactness of perturbations). The linear operators

$$
A^{1}: H_{a}^{l+2 m-1}(Q) \rightarrow H_{a}^{l}(Q), \quad B_{i \mu}^{3}: H_{a}^{l+2 m-1}(Q) \rightarrow H_{a}^{l+2 m-m_{i \mu}-1 / 2}\left(\Gamma_{i}\right)
$$

are bounded $\left(i=1, \ldots, N_{0} ; \mu=1, \ldots, m\right)$.

Condition 6.2 (Separability from the conjugation points). The linear operators $B_{i \mu}^{2}$ : $H_{a}^{l+2 m}(Q) \rightarrow H_{a}^{l+2 m-m_{i \mu}-1 / 2}\left(\Gamma_{i}\right)$ are bounded, and there exist numbers $\sigma>0$ and $\varkappa_{1}>$ $\varkappa_{2}>0$ such that

$$
\left\|B_{i \mu}^{2} u\right\|_{H_{a}^{l+2 m-m_{i \mu}-1 / 2}\left(\Gamma_{i}\right)} \leq c_{1}\|u\|_{H_{a}^{l+2 m}\left(Q \backslash \overline{\mathcal{K}_{1}^{\varkappa 1}}\right)}
$$

for all $u \in H_{a}^{l+2 m}\left(Q \backslash \overline{\mathcal{K}_{1}^{\varkappa_{1}}}\right)$ and

$$
\left\|B_{i \mu}^{2} u\right\|_{H_{a}^{l+2 m-m_{i \mu}-1 / 2}\left(\Gamma_{i} \backslash \overline{\mathcal{K}_{1}^{\varkappa 2}}\right)} \leq c_{2}\|u\|_{H_{a}^{l+2 m}\left(Q_{\sigma}\right)}
$$

for all $u \in H_{a}^{l+2 m}\left(Q_{\sigma}\right)$; here $i=1, \ldots, N_{0} ; \mu=1, \ldots, m ; c_{1}, c_{2}>0$ do not depend on $u$.

Remark 6.1. It follows from Lemma 4.6 that problem (1.3), (1.4) can be represented in the form (6.1), (6.2) with the operators $A^{1}, B_{i \mu}^{2}$, and $B_{i \mu}^{3}$ satisfying Conditions 6.1 and 6.2 . 
Remark 6.2. The proofs of Theorems 4.1 and 5.2 use only Conditions 6.1 and 6.2, rather than any explicit form of the operators $A^{1}, B_{i \mu}^{2}$, and $B_{i \mu}^{3}$. To prove that those operators satisfy Conditions 6.1 and 6.2, we used Condition 4.1, which provided the choice of the set $K$ and the number $a$ in Secs. 1 15. However, Condition 4.1 is needless in this section because the fulfilment of Conditions 6.1 and 6.2 is postulated rather than proved.

6.2. We introduce the linear bounded operator

$$
\mathbf{L}=\left\{A, B_{i \mu}\right\}: H_{a}^{l+2 m}(Q) \rightarrow \mathcal{H}_{a}^{l}(Q, \Gamma)
$$

corresponding to problem (6.1), (6.2).

For every fixed point $g \in \mathcal{K}_{1}$, we consider the linear bounded operator $\mathcal{L}_{g}(\omega)$ : $\mathcal{E}_{a}^{l+2 m}(\theta) \rightarrow \mathcal{E}_{a}^{l}(\theta, \gamma)$ given by (2.1), where $\omega \in S^{n-3}$. We also consider the analytic operator-valued function $\hat{\mathcal{L}}_{g}(\lambda): \mathcal{W}^{l+2 m}\left(d_{1}, d_{2}\right) \rightarrow \mathcal{W}^{l}\left[d_{1}, d_{2}\right]$ given by (2.2).

For every fixed point $g \in K_{2}$, we consider the linear bounded operator $\mathcal{L}_{g}(\omega)$ : $E_{a}^{l+2 m}\left(\mathbb{R}_{+}^{2}\right) \rightarrow \mathcal{E}_{a}^{l}\left(\mathbb{R}_{+}^{2}, \gamma\right)$ given by (2.26), where $\omega \in S^{n-3}$. We also consider the analytic operator-valued function $\hat{\mathcal{L}}_{g}(\lambda): W^{l+2 m}(-\pi / 2, \pi / 2) \rightarrow \mathcal{W}^{l}[-\pi / 2, \pi / 2]$ given by (2.27).

Similarly to the proofs of Theorems 4.1 and 5.2, using Conditions 6.1 and 6.2 and assuming that the number $\varepsilon>0$ in the definition of the function $\xi$ is sufficiently small, we obtain the following two results.

Theorem 6.1. Let Conditions 1.1 1.4, 6.1, and 6.2 hold. Assume that the line $\operatorname{Im} \lambda=$ $a+1-l-2 m$ contains no eigenvalues of $\hat{\mathcal{L}}_{g}(\lambda)$ for any $g \in K$ and $\operatorname{dim} \mathcal{N}\left(\mathcal{L}_{g}(\omega)\right)=$ $\operatorname{codim} \mathcal{R}\left(\mathcal{L}_{g}(\omega)\right)=0$ for any $g \in K$ and $\omega \in S^{n-3}$. Then the following estimate holds for all $u \in H_{a}^{l+2 m}(Q)$ :

$$
\|u\|_{H_{a}^{l+2 m}(Q)} \leq c\left(\|\mathbf{L} u\|_{\mathcal{H}_{a}^{l}(Q, \Gamma)}+\|u\|_{H_{a}^{l+2 m-1}(Q)}\right),
$$

where $c>0$ does not depend on $u$.

Theorem 6.2. Let the conditions of Theorem 6.1 be fulfilled. Then there exists a linear bounded operator $\mathbf{R}: \mathcal{H}_{a}^{l}(Q, \Gamma) \rightarrow H_{a}^{l+2 m}(Q)$ such that

$$
\mathbf{L R}=\mathbf{I}+\mathbf{T}
$$

where $\mathbf{T}: \mathcal{H}_{a}^{l}(Q, \Gamma) \rightarrow \mathcal{H}_{a}^{l}(Q, \Gamma)$ is a compact operator.

Along with the operator $\mathbf{L}$, we consider the bounded operator

$$
\mathbf{L}^{1}=\left\{A^{0}, B_{i \mu}^{0}+B_{i \mu}^{1}\right\}: H_{a}^{l+2 m}(Q) \rightarrow \mathcal{H}_{a}^{l}(Q, \Gamma) .
$$

It follows from Theorem 5.1 that the operator $\mathbf{L}^{1}$ has the Fredholm property.

Similarly to the proofs of Theorem [5.1 and Corollary [5.1 using Theorems 6.1 and 6.2, we obtain the following result.

Theorem 6.3. Let the conditions of Theorem 6.1 be fulfilled. Then the operator $\mathbf{L}$ : $H_{a}^{l+2 m}(Q) \rightarrow \mathcal{H}_{a}^{l}(Q, \Gamma)$ has the Fredholm property and ind $\mathbf{L}=\operatorname{ind} \mathbf{L}^{1}$.

Theorem 6.3 shows that the addition of the operators $A^{1}, B_{i \mu}^{2}$, and $B_{i \mu}^{3}$ satisfying Conditions 6.1 and 6.2 neither violates the Fredholm property nor changes the index.

6.3. Now we consider an example of an elliptic problem with distributed nonlocal terms satisfying Condition 6.2

Example 6.1. Let $Q \subset \mathbb{R}^{3}$ be a bounded domain with a boundary $\partial Q$ that is a surface of revolution about the axis $x_{3}$. Denote $P=\{0,0,1\} \cup\{0,0,-1\}$. Assume that, outside $P^{1 / 4}$, the surface $\partial Q$ coincides with the boundary of the domain

$$
\left\{x: x_{3}<1-\sqrt{x_{1}^{2}+x_{2}^{2}}\right\} \cap\left\{x: x_{3}>-1+\sqrt{x_{1}^{2}+x_{2}^{2}}\right\} .
$$


Denote

$$
\Gamma_{1}=\left\{x \in \partial Q: x_{3}<0\right\}, \quad \Gamma_{2}=\left\{x \in \partial Q: x_{3}>0\right\} .
$$

In this case, we have

$$
\mathcal{K}_{1}=\left\{x: x_{1}^{2}+x_{2}^{2}=1, x_{3}=0\right\} .
$$

Assume that the boundary $\partial Q$ is infinitely smooth outside the set $\mathcal{K}_{1}$.

We introduce the operators

$$
B_{l}^{1} u=-\left.\alpha_{l}(\xi u)\left(\omega_{l}(x)\right)\right|_{\Gamma_{l}}, \quad l=1,2 .
$$

The transformations $\omega_{l}(x)$ in (6.5) are defined for $x \in \mathcal{K}_{1}^{\varepsilon_{0}}$ by the formula

$$
\begin{aligned}
\omega_{l}(x)= & \left(\cos \varphi\left[1-\frac{1}{\sqrt{2}}\left((1-r)+(-1)^{l} x_{3}\right)\right]\right. \\
& \left.\sin \varphi\left[1-\frac{1}{\sqrt{2}}\left((1-r)+(-1)^{l} x_{3}\right)\right], \frac{1}{\sqrt{2}}\left[(-1)^{l+1}(1-r)+x_{3}\right]\right),
\end{aligned}
$$

where $r, \varphi, x_{3}$ are the cylindrical coordinates of the point $x$, the number $\varepsilon_{0}>0$ is sufficiently small, the function $\xi \in C_{0}^{\infty}\left(\mathbb{R}^{3}\right)$ is such that $\xi(x)=1$ for $x \in \mathcal{K}_{1}^{\varepsilon / 2}$ and $\operatorname{supp} \xi \subset \mathcal{K}_{1}^{\varepsilon}, 0<\varepsilon \leq \varepsilon_{0}$, and $\alpha_{1}, \alpha_{2} \in \mathbb{R}$. Clearly, we have $\omega_{l}\left(\mathcal{K}_{1}\right)=\mathcal{K}_{1}$.

Since $\Gamma_{l} \in C^{\infty}$, one can find a sufficiently small $\varkappa>0$ possessing the following property: for any $x \in \Gamma_{l}^{5 \varkappa} \cap Q$, there exists a unique pair $(y, t), y \in \Gamma_{l}, t>0$, such that $x=y+n_{y} t$, where $n_{y}$ denotes the unit normal to $\Gamma_{l}$ at the point $y$, directed inside the domain $Q$. One can show that the transformation $x \mapsto(y, t)$ is a $C^{\infty}$ diffeomorphism mapping $\Gamma_{l}^{5 \varkappa} \cap Q$ onto $\Gamma_{l} \times(0,5 \varkappa)$, provided that $\varkappa>0$ is sufficiently small.

Introduce a function $\eta \in C_{0}^{\infty}(\mathbb{R})$ such that $\eta(t)=1$ for $t \in(3 \varkappa, 4 \varkappa)$ and $\operatorname{supp} \eta \subset$ $(2 \varkappa, 5 \varkappa)$. Consider the operators

$$
B_{l}^{2} u=\left.F^{-1}\left(b_{l} F(\eta u)\right)\right|_{t=0}, \quad l=1,2,
$$

where

$$
F(\eta u)(y, \lambda)=\frac{1}{\sqrt{2 \pi}} \int_{\mathbb{R}} e^{-i \lambda t} \eta(t) u(y, t) d t
$$

is the Fourier transform with respect to $t, F^{-1}$ is the inverse Fourier transform, and $b_{l}(\lambda)$ is a function continuous on $\mathbb{R}$ such that

$$
\sup _{\lambda \in \mathbb{R}}\left|b_{l}(\lambda)\right|<\infty .
$$

We consider the following nonlocal boundary-value problem:

$$
\begin{gathered}
-\Delta u=f_{0}(x), \quad x \in Q, \\
\left.u\right|_{\Gamma_{l}}+B_{l}^{1} u+B_{l}^{2} u=f_{l}(x), \quad x \in \Gamma_{l}, l=1,2 .
\end{gathered}
$$

We set $K=\mathcal{K}_{1}$ in the definition of the space $H_{a}^{l}(Q)=H_{a}^{l}(Q, K)$.

The Jacobian $\frac{D \omega_{l}}{D x}$ can be calculated on $\mathcal{K}_{1}$ as follows:

$$
\left.\frac{D \omega_{l}}{D x}\right|_{\mathcal{K}_{1}}=\left.\left.\frac{D \omega_{l}}{D\left(r, \varphi, x_{3}\right)}\right|_{\mathcal{K}_{1}} \frac{D\left(r, \varphi, x_{3}\right)}{D x}\right|_{\mathcal{K}_{1}}
$$


Since $\left.\frac{D\left(r, \varphi, x_{3}\right)}{D x}\right|_{\mathcal{K}_{1}}=1$, we have

$$
\begin{aligned}
\left.\frac{D \omega_{l}}{D x}\right|_{\mathcal{K}_{1}} & =\left.\operatorname{det}\left(\begin{array}{lll}
\frac{\partial \omega_{l 1}}{\partial r} & \frac{\partial \omega_{l 1}}{\partial \varphi} & \frac{\partial \omega_{l 1}}{\partial x_{3}} \\
\frac{\partial \omega_{l 2}}{\partial r} & \frac{\partial \omega_{l 2}}{\partial \varphi} & \frac{\partial \omega_{l 2}}{\partial x_{3}} \\
\frac{\partial \omega_{l 3}}{\partial r} & \frac{\partial \omega_{l 3}}{\partial \varphi} & \frac{\partial \omega_{l 3}}{\partial x_{3}}
\end{array}\right)\right|_{\mathcal{K}_{1}} \\
& =\operatorname{det}\left(\begin{array}{ccc}
\frac{1}{\sqrt{2}} \cos \varphi & -\sin \varphi & \frac{1}{\sqrt{2}}(-1)^{l+1} \cos \varphi \\
\frac{1}{\sqrt{2}} \sin \varphi & \cos \varphi & \frac{1}{\sqrt{2}}(-1)^{l+1} \sin \varphi \\
\frac{1}{\sqrt{2}}(-1)^{l} & 0 & \frac{1}{\sqrt{2}}
\end{array}\right)=1
\end{aligned}
$$

Therefore, we can choose $\varepsilon_{0}>0$ so small that $\omega_{l}: \mathcal{K}_{1}^{\varepsilon_{0}} \rightarrow \omega_{l}\left(\mathcal{K}_{1}^{\varepsilon_{0}}\right)$ is a $C^{\infty}$ diffeomorphism. Furthermore, $\omega_{l}\left(\mathcal{K}_{1}^{\varepsilon_{0}}\right)=\mathcal{K}_{1}^{\varepsilon_{0}}$ and $\omega_{l}\left(\Gamma_{l} \cap \mathcal{K}_{1}^{\varepsilon_{0}}\right)=\left\{x: 1-\varepsilon_{0}<r<1, x_{3}=0\right\} \subset Q$.

Since $\omega_{l}(g)=g$ for $g \in \mathcal{K}_{1}$ and $l=1,2$, the orbit of each point $g \in \mathcal{K}_{1}$ consists of one point $g_{1}=g$. For $x \neq 0$, we introduce the new variables $x^{\prime}=\left(y^{\prime}, z^{\prime}\right)$ given by

$$
y_{1}^{\prime}=1-r, \quad y_{2}^{\prime}=x_{3}, \quad z^{\prime}=\varphi-\varphi_{1},
$$

where $y^{\prime}=\left(y_{1}^{\prime}, y_{2}^{\prime}\right)$, while $r, \varphi, x_{3}$ and $1, \varphi_{1}, 0$ are the cylindrical coordinates of the points $x$ and $g_{1}$, respectively.

Set $V(0)=\left\{x^{\prime}:\left|y^{\prime}\right|<\varepsilon_{1},\left|z^{\prime}\right|<\varepsilon_{1}\right\}$, where $\varepsilon_{1}<\min \left\{\varepsilon_{0}, 2 \varkappa\right\}$. Let $\hat{V}\left(g_{1}\right)=V\left(g_{1}\right)$ be the pre-image of the set $V(0)$ under the change of variables $x \mapsto x^{\prime}$. Assume that supp $u \subset V\left(g_{1}\right) \cap \bar{Q}$. Introduce the function $v\left(x^{\prime}\right)=u\left(x\left(x^{\prime}\right)\right)$. Denote $x^{\prime}=\left(y^{\prime}, z^{\prime}\right)$ by $x=\left(y_{1}, y_{2}, z\right)$. Then the boundary-value problem (6.9), (6.10) takes the form

$$
\begin{gathered}
-\frac{\partial^{2} v}{\partial y_{1}^{2}}-\frac{\partial^{2} v}{\partial y_{2}^{2}}-\frac{1}{\left(1-y_{1}\right)^{2}} \frac{\partial^{2} v}{\partial z^{2}}-\frac{1}{1-y_{1}} \frac{\partial v}{\partial y_{1}}=f_{0}^{\prime}(x), \quad x \in \Theta, \\
\left.v(y, z)\right|_{\Gamma_{1 l}}-\left.\alpha_{l} v\left(\mathcal{G}_{l} y, z\right)\right|_{\Gamma_{1 l}}=f_{l}^{\prime}(x), \quad x \in \Gamma_{1 l}, \quad l=1,2,
\end{gathered}
$$

where $\mathcal{G}_{l}$ is the operator of rotation by the angle $(-1)^{l+1} \pi / 4$,

$$
\Theta=\{x: r>0,|\varphi|<\pi / 4, z \in \mathbb{R}\}, \quad \Gamma_{1 l}=\left\{x: r>0, \varphi=(-1)^{l} \pi / 4, z \in \mathbb{R}\right\},
$$

and $r, \varphi$ are the polar coordinates of the point $y$.

Clearly, Conditions 1.1 1.4 are fulfilled in this example.

Passing to the principal homogeneous part in (6.11) with the coefficients frozen at the origin, we obtain

$$
-\Delta v=f_{0}^{\prime}(x), \quad x \in \Theta .
$$

The nonlocal boundary conditions (6.12) do not change.

In the case of problem (6.13), (6.12), the operator $\mathcal{L}_{g}=\mathcal{L}: H_{a}^{2}(\Theta) \rightarrow \mathcal{H}_{a}^{0}(\Theta, \Gamma)$ given by (1.11) takes the form

$\mathcal{L} v=\left(-\Delta v,\left.v(\varphi, r, z)\right|_{\Gamma_{11}}-\left.\alpha_{1} v(\varphi+\pi / 4, r, z)\right|_{\Gamma_{11}},\left.v(\varphi, r, z)\right|_{\Gamma_{12}}-\left.\alpha_{2} v(\varphi-\pi / 4, r, z)\right|_{\Gamma_{12}}\right)$.

Hence, the operators

$$
\mathcal{L}_{g}(\omega)=\mathcal{L}(\omega): E_{a}^{2}(\theta) \rightarrow \mathcal{E}_{a}^{0}(\theta, \gamma)
$$

and

$$
\hat{\mathcal{L}}_{g}(\lambda)=\hat{\mathcal{L}}(\lambda): W^{2}(-\pi / 4, \pi / 4) \rightarrow \mathcal{W}^{0}[-\pi / 4, \pi / 4]
$$


given by (2.1) and (2.2) have the form

$$
\begin{array}{r}
\mathcal{L}(\omega) V=\left(-\Delta_{y} V+V,\left.V(\varphi, r)\right|_{\gamma_{11}}-\left.\alpha_{1} V(\varphi+\pi / 4, r)\right|_{\gamma_{11}},\right. \\
\left.\left.V(\varphi, r)\right|_{\gamma_{12}}-\left.\alpha_{2} V(\varphi-\pi / 4, r)\right|_{\gamma_{12}}\right)
\end{array}
$$

and

$$
\hat{\mathcal{L}}(\lambda) w=\left(-w_{\varphi \varphi}+\lambda^{2} w, w(-\pi / 4)-\alpha_{1} w(0), w(\pi / 4)-\alpha_{2} w(0)\right),
$$

respectively, where

$$
\theta=\left\{y \in \mathbb{R}^{2}: r>0,|\varphi|<\pi / 4\right\}, \quad \gamma_{1 l}=\left\{y \in \mathbb{R}^{2}: r>0, \varphi=(-1)^{l} \pi / 4\right\},
$$

and $\omega= \pm 1$ (cf. Example 2.1 for $d=\pi / 2$ ).

It follows from Example 2.1 that the strip $-1 \leq \operatorname{Im} \lambda \leq 1$ contains no eigenvalues of the operator-valued function $\hat{\mathcal{L}}(\lambda)$ and the operator $\mathcal{L}(\omega), \omega= \pm 1$, is an isomorphism for $0 \leq$ $a \leq 2$ and $\alpha_{1}, \alpha_{2} \in \mathbb{R}$ such that $0<\left|\alpha_{1}+\alpha_{2}\right|<2$ and $\pi / 4<\arctan \sqrt{4\left(\alpha_{1}+\alpha_{2}\right)^{-2}-1}$. Therefore, by Theorem 2.2, the operator $\mathcal{L}$ is an isomorphism for the above $a$ and $\alpha_{l}$.

Suppose that $a>1$ and prove that the operators $B_{l}^{2}$ satisfy Condition 6.2 with $\varkappa_{1}=2 \varkappa, \varkappa_{2}=\varkappa$, and $\sigma=\varkappa$. Using Lemma 4.5, we have

$$
\begin{aligned}
\left\|B_{l}^{2} u\right\|_{H_{a}^{3 / 2}\left(\Gamma_{l}\right)} & \leq k_{1}\left\|F^{-1}\left(b_{l} F(\eta u)\right)\right\|_{W^{2}\left(\Gamma_{l} \times(-\infty, \infty)\right)} \\
& =\frac{k_{1}}{\sqrt{2 \pi}} \sum_{|\alpha|+\beta \leq 2}\left\|D_{y}^{\alpha} D_{t}^{\beta} \int_{\mathbb{R}} e^{i \lambda t} b_{l}(\lambda) F(\eta u)(y, \lambda) d \lambda\right\|_{L_{2}\left(\Gamma_{l} \times(-\infty, \infty)\right)} \\
& =\frac{k_{1}}{\sqrt{2 \pi}} \sum_{|\alpha|+\beta \leq 2}\left\|\int_{\mathbb{R}} e^{i \lambda t} \lambda^{\beta} b_{l}(\lambda) D_{y}^{\alpha} F(\eta u)(y, \lambda) d \lambda\right\|_{L_{2}\left(\Gamma_{l} \times(-\infty, \infty)\right)},
\end{aligned}
$$

where $D_{t}=-i \partial / \partial t$. Applying the Plancherel theorem and using property (6.8), we obtain

$$
\begin{aligned}
\left\|B_{l}^{2} u\right\|_{H_{a}^{3 / 2}\left(\Gamma_{l}\right)} & \leq \frac{k_{1}}{\sqrt{2 \pi}} \sum_{|\alpha|+\beta \leq 2}\left\|\lambda^{\beta} b_{l}(\lambda) D_{y}^{\alpha} F(\eta u)(y, \lambda)\right\|_{L_{2}\left(\Gamma_{l} \times(-\infty, \infty)\right)} \\
& \leq k_{2} \sum_{|\alpha|+\beta \leq 2}\left\|\lambda^{\beta} D_{y}^{\alpha} F(\eta u)(y, \lambda)\right\|_{L_{2}\left(\Gamma_{l} \times(-\infty, \infty)\right)} .
\end{aligned}
$$

Applying the Plancherel theorem once more and taking into account that $\operatorname{supp} \eta \subset$ $(2 \varkappa, 5 \varkappa)$, we have

$$
\left\|B_{l}^{2} u\right\|_{H_{a}^{3 / 2}\left(\Gamma_{l}\right)} \leq k_{3}\|u\|_{W^{2}\left(\Omega_{l \varkappa}^{1}\right)},
$$

where

$$
\Omega_{l \varkappa}^{1}=\left\{x=y+n_{y} t: y \in \Gamma_{l}, t \in(2 \varkappa, 5 \varkappa)\right\}
$$

(see Figure 4). Since $\Omega_{l \varkappa}^{1} \subset Q \backslash \overline{\mathcal{K}_{1}^{2 \varkappa}}$ and the norms in $W^{2}\left(Q \backslash \overline{\mathcal{K}_{1}^{2 \varkappa}}\right)$ and $H_{a}^{2}\left(Q \backslash \overline{\mathcal{K}_{1}^{2 \varkappa}}\right)$ are equivalent, the last inequality yields

$$
\left\|B_{l}^{2} u\right\|_{H_{a}^{3 / 2}\left(\Gamma_{l}\right)} \leq k_{4}\|u\|_{H_{a}^{2}\left(Q \backslash \overline{\mathcal{K}_{1}^{2 \varkappa}}\right)} .
$$

Denote

$$
\Omega_{l \varkappa}^{2}=\left\{x=y+n_{y} t: y \in \Gamma_{l} \backslash \overline{\mathcal{K}_{1}^{\varkappa}}, t \in(2 \varkappa, 5 \varkappa)\right\} .
$$

Since $\Omega_{l \varkappa}^{2} \subset Q_{\varkappa}$, similarly to (6.17), we obtain

$$
\left\|B_{l}^{2} u\right\|_{H_{a}^{3 / 2}\left(\Gamma_{l} \backslash \overline{\mathcal{K}_{1}^{\varkappa}}\right)} \leq k_{5}\|u\|_{H_{a}^{2}\left(Q_{\varkappa}\right)} .
$$

It follows from (6.17) and (6.18) that the operators $B_{l}^{2}$ satisfy Condition 6.2 for $a>1$. We consider the linear bounded operators

$$
\mathbf{L}, \mathbf{L}^{1}: H_{a}^{2}(Q) \rightarrow H_{a}^{0}(Q) \times H_{a}^{3 / 2}\left(\Gamma_{1}\right) \times H_{a}^{3 / 2}\left(\Gamma_{2}\right)
$$



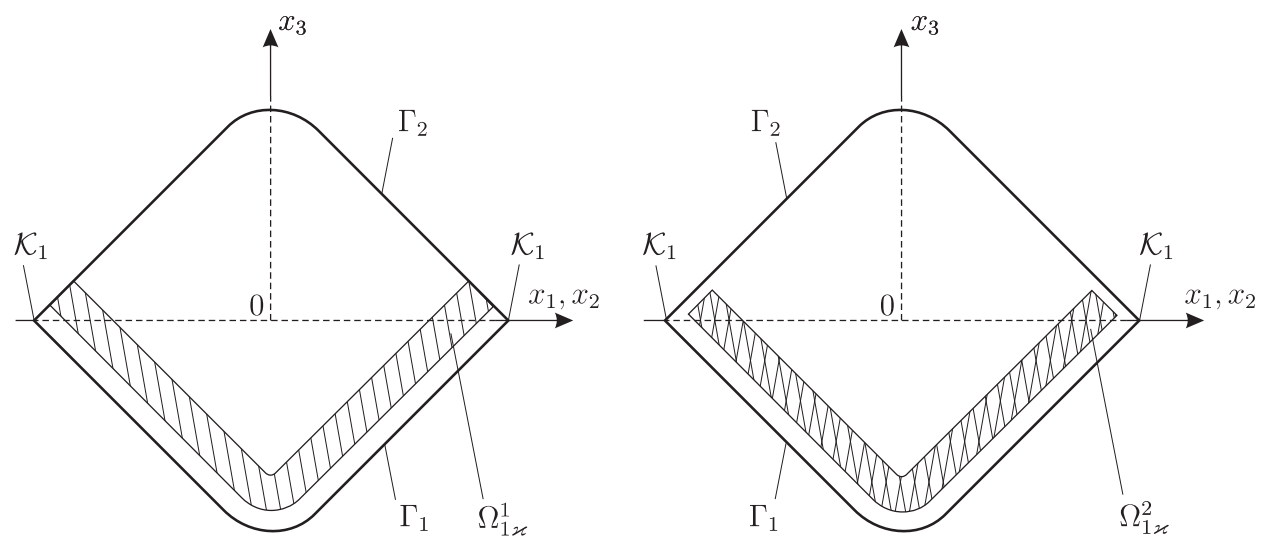

Figure 4. Problem (6.9), (6.10)

given by

$$
\mathbf{L} u=\left\{-\Delta u,\left.u\right|_{\Gamma_{l}}+B_{l}^{1} u+B_{l}^{2} u\right\}, \quad \mathbf{L}^{1} u=\left\{-\Delta u,\left.u\right|_{\Gamma_{l}}+B_{l}^{1} u\right\} .
$$

It follows from Theorem 5.1 that the operator $\mathbf{L}^{1}$ has the Fredholm property for $0 \leq$ $a \leq 2$ and $\alpha_{1}, \alpha_{2} \in \mathbb{R}$ such that $0<\left|\alpha_{1}+\alpha_{2}\right|<2$ and $\pi / 4<\arctan \sqrt{4\left(\alpha_{1}+\alpha_{2}\right)^{-2}-1}$. By Theorem 6.3. the operator $\mathbf{L}$ has the Fredholm property and ind $\mathbf{L}=$ ind $\mathbf{L}^{1}$ for $1<a \leq 2, \alpha_{1}, \alpha_{2} \in \mathbb{R}$ such that $0<\left|\alpha_{1}+\alpha_{2}\right|<2$ and $\pi / 4<\arctan \sqrt{4\left(\alpha_{1}+\alpha_{2}\right)^{-2}-1}$, and for a continuous function $b(\lambda)$ satisfying relation (6.8).

\section{Setting of NONLOCAL ELLIPTIC PROBLEMS WITH A PARAMETER MODEL OPERATOR}

7.1. In Secs. 7 and 8 we prove the unique solvability of nonlocal elliptic problems with a parameter $p=\left(p_{1}, \ldots, p_{d}\right) \in \mathbb{R}^{d}$, where $d \geq 1$. Similarly to the above, we first establish the unique solvability of model nonlocal problems with a parameter in dihedral angles. Combining these results with those in 22 and 20] and making use of a partition of unity, we will consider nonlocal problems in bounded domains.

Let the domain $Q$, the transformations $\omega_{i s}$, and the sets $\mathcal{K}_{1}, K_{2}$, and $K$ be the same as in Sec. 6.

To consider nonlocal problems with a parameter, we introduce norms for the weighted spaces, depending on this parameter. First, we introduce the norms on the dihedral angle

$$
\Theta=\left\{x=(y, z) \in \mathbb{R}^{n}: d_{1}<\varphi<d_{2}, z \in \mathbb{R}^{n-2}\right\}
$$

and on the half-plane

$$
\Gamma=\left\{x=(y, z) \in \mathbb{R}^{n}: \varphi=d^{\prime}, z \in \mathbb{R}^{n-2}\right\}, \quad d_{1} \leq d^{\prime} \leq d_{2} .
$$

Consider the space $V_{a}^{k}(\Theta)=H_{a}^{k}(\Theta) \cap H_{a}^{0}(\Theta)$ with the norm

$$
\|u\|_{V_{a}^{k}(\Theta)}=\left(\|u\|_{H_{a}^{k}(\Theta)}^{2}+|p|^{2 k}\|u\|_{H_{a}^{0}(\Theta)}^{2}\right)^{1 / 2} \quad\left(u \in V_{a}^{k}(\Theta)\right),
$$

where $k \geq 0$ is an integer.

For an integer $\nu \geq 0$, we denote

$$
\|\psi\|_{H_{a}^{\nu}(\Gamma)}^{2}=\sum_{|\alpha| \leq \nu} \int_{\Gamma} r^{2(a-\nu+|\alpha|)}\left|D^{\alpha} \psi\right|^{2} d \Gamma .
$$


Consider the space $V_{a}^{k-1 / 2}(\Gamma)=H_{a}^{k-1 / 2}(\Gamma) \cap H_{a}^{0}(\Gamma)$ with the norm

$$
\|\psi\|_{V_{a}^{k-1 / 2}(\Gamma)}=\left(\|\psi\|_{H_{a}^{k-1 / 2}(\Gamma)}^{2}+|p|^{2(k-1 / 2)}\|\psi\|_{H_{a}^{0}(\Gamma)}^{2}\right)^{1 / 2} \quad\left(\psi \in V_{a}^{k-1 / 2}(\Gamma), k \geq 1\right) .
$$

Now we introduce the norms for the domain $Q$ and for the manifolds $\Gamma_{i}$. Set2

$$
\begin{gathered}
\|u\|_{H_{a}^{k}(Q)}=\left(\|u\|_{H_{a}^{k}(Q)}^{2}+|p|^{2 k}\|u\|_{H_{a}^{0}(Q)}^{2}\right)^{1 / 2} \quad\left(u \in H_{a}^{k}(Q)\right), \\
\|\psi\|_{H_{a}^{k-1 / 2}\left(\Gamma_{i}\right)}=\left(\|\psi\|_{H_{a}^{k-1 / 2}\left(\Gamma_{i}\right)}^{2}+|p|^{2(k-1 / 2)}\|\psi\|_{H_{a}^{0}\left(\Gamma_{i}\right)}^{2}\right)^{1 / 2} \quad\left(\psi \in H_{a}^{k-1 / 2}\left(\Gamma_{i}\right), k \geq 1\right),
\end{gathered}
$$

where

$$
\|\psi\|_{H_{a}^{0}\left(\Gamma_{i}\right)}^{2}=\int_{\Gamma_{i}} \rho^{2 a}|\psi|^{2} d \Gamma_{i}
$$

and $\rho(x)$ is the function occurring in the definition of the spaces $H_{a}^{k}(Q)$.

We also set

$$
\|f\|_{\mathcal{H}_{a}^{l}(Q, \Gamma)}=\left(\left\|f_{0}\right\|_{H_{a}^{l}(Q)}^{2}+\sum_{i, \mu}\left\|f_{i \mu}\right\|_{H_{a}^{l+2 m-m_{i \mu}-1 / 2}\left(\Gamma_{i}\right)}^{2}\right)^{1 / 2}
$$

for $f=\left\{f_{0}, f_{i \mu}\right\} \in \mathcal{H}_{a}^{l}(Q, \Gamma)$.

Lemma 7.1. (1) For all $u \in V_{a}^{k}(\Theta), k \geq 2$ is an integer, $p \in \mathbb{R}^{d}$, and integer $s$, $0<s<k$, we have

$$
|p|^{k-s}\|u\|_{H_{a}^{s}(\Theta)} \leq c_{1}\|u\|_{V_{a}^{k}(\Theta)},
$$

where $\Theta$ is defined in (7.1) and $c_{1}=c_{1}(k, s)>0$ does not depend on $u$ or $p$.

(2) For all $u \in H_{a}^{k}(Q), k \geq 2$ is an integer, $p \in \mathbb{R}^{d}$, and integer $s, 0<s<k$, we have

$$
|p|^{k-s}\|u\|_{H_{a}^{s}(Q)} \leq c_{2}\|u\|_{H_{a}^{k}(Q)},
$$

where $c_{2}=c_{2}(k, s)>0$ does not depend on $u$ or $p$.

Proof. First, we prove the interpolation inequality (7.5).

Let $\left\{\xi_{l}\right\}_{l=-\infty}^{+\infty}$ be a partition of unity subordinate to the covering of the angle $\theta=\{y \in$ $\left.\mathbb{R}^{2}: d_{1}<\varphi<d_{2}\right\}$ by the sets $\theta_{l}=\left\{y \in \theta: 2^{l-1}<r<2^{l+1}\right\}$ such that

$$
\left|D^{\alpha} \xi_{l}(y)\right| \leq k_{\alpha} 2^{-|\alpha| l}, \quad y \in \theta_{l}, l=0, \pm 1, \pm 2, \ldots,
$$

where $k_{\alpha}>0$ does not depend on $l$.

Denote $\Theta_{l}=\theta_{l} \times \mathbb{R}^{n-2}, l=0, \pm 1, \pm 2, \ldots$.

Using (7.7) and the fact that $\operatorname{supp} \xi_{l} \cap \overline{\Theta_{j}}=\varnothing$ for $j \neq l-1, l, l+1$, one can easily verify that

$$
\|u\|_{H_{a}^{s}(\Theta)} \approx\left(\sum_{l=-\infty}^{+\infty}\left\|\xi_{l} u\right\|_{H_{a}^{s}\left(\Theta_{l}\right)}^{2}\right)^{1 / 2}
$$

for $s=0,1,2, \ldots$, where the symbol $\approx$ means the equivalence of the norms.

Using the theorem on the extension of functions from a domain with Lipschitz boundary to $\mathbb{R}^{n}$ and applying the interpolation inequality for the Sobolev space $W^{k}\left(\mathbb{R}^{n}\right)$ (see 2 , Sec. 1]), we obtain

$$
q^{2(k-s)}\|v\|_{W^{s}\left(\Theta_{0}\right)}^{2} \leq k_{1}\left(\|v\|_{W^{k}\left(\Theta_{0}\right)}^{2}+q^{2 k}\|v\|_{L_{2}\left(\Theta_{0}\right)}^{2}\right)
$$

for all $v \in W^{k}\left(\Theta_{0}\right)$ and $q>0$, where $k_{1}>0$ does not depend on $v$ or $q$.

\footnotetext{
${ }^{2}$ We do not introduce the spaces $V_{a}^{k}(Q)=H_{a}^{k}(Q) \cap H_{a}^{0}(Q)$ and $V_{a}^{k-1 / 2}\left(\Gamma_{i}\right)=H_{a}^{k-1 / 2}\left(\Gamma_{i}\right) \cap H_{a}^{0}\left(\Gamma_{i}\right)$ because they coincide with $H_{a}^{k}(Q)$ and $H_{a}^{k-1 / 2}\left(\Gamma_{i}\right)$, respectively, in the case of a bounded domain $Q$.
} 
We introduce the new variables $x^{\prime}=2^{-l} x$. Using the equivalence of the norms (7.8) and the interpolation inequality (7.9) with $q=|p| 2^{l}$ and reverting to the variables $x=$ $2^{l} x^{\prime}$, we have

$$
\begin{aligned}
& |p|^{2(k-s)}\|u\|_{H_{a}^{s}(\Theta)}^{2} \leq k_{2}|p|^{2(k-s)} \sum_{l=-\infty}^{+\infty} 2^{(2(a-s)+n) l} \sum_{|\alpha| \leq s} \int_{\Theta_{0}}\left|D_{x^{\prime}}^{\alpha}\left(\xi_{l} u\right)\left(x^{\prime}\right)\right|^{2} d x^{\prime} \\
& \quad \leq k_{3} \sum_{l=-\infty}^{+\infty} 2^{(2(a-k)+n) l}\left(\sum_{|\alpha| \leq k} \int_{\Theta_{0}}\left|D_{x^{\prime}}^{\alpha}\left(\xi_{l} u\right)\left(x^{\prime}\right)\right|^{2} d x^{\prime}+|p|^{2 k} 2^{2 l k} \int_{\Theta_{0}}\left|\left(\xi_{l} u\right)\left(x^{\prime}\right)\right|^{2} d x^{\prime}\right) \\
& \quad \leq k_{4} \sum_{l=-\infty}^{+\infty}\left(\left\|\xi_{l} u\right\|_{H_{a}^{k}\left(\Theta_{l}\right)}^{2}+|p|^{2 k}\left\|\xi_{l} u\right\|_{H_{a}^{0}\left(\Theta_{l}\right)}^{2}\right),
\end{aligned}
$$

where $k_{2}, k_{3}, k_{4}>0$ do not depend on $u$ or $p$. Combining this inequality with (7.8) yields (7.5).

2. Note that the relation $u \in H_{a}^{k}(\Theta)$ implies that $u \in V_{a}^{k}(\Theta)$, provided that $u$ is compactly supported. Therefore, using a partition of unity, interpolation inequality (7.5), and the interpolation inequality of the kind (7.9) for Sobolev spaces, we obtain (7.6) for all $u \in H_{a}^{k}(Q)$ and $p \in \mathbb{R}^{d}$.

Lemma 7.2. (1) For all $u \in V_{a}^{1}(\Theta)$ and $p \in \mathbb{R}^{d}$, we have

$$
|p|^{1 / 2}\left\|\left.u\right|_{\Gamma}\right\|_{H_{a}^{0}(\Gamma)} \leq c_{1}\|u\|_{V_{a}^{1}(\Theta)},
$$

where $\Theta$ and $\Gamma$ are defined in (7.1) and (7.2) respectively, while $c_{1}>0$ does not depend on $u$ or $p$.

(2) For all $u \in H_{a}^{1}(Q)$ and $p \in \mathbb{R}^{d}$, we have

$$
|p|^{1 / 2}\left\|\left.u\right|_{\Gamma_{i}}\right\|_{H_{a}^{0}\left(\Gamma_{i}\right)} \leq c_{2}\|u\|_{H_{a}^{1}(Q)},
$$

where $c_{2}>0$ does not depend on $u$ or $p$.

Proof. Similarly to the proof of Lemma 7.1, it suffices to prove inequality (7.10).

Denote $\Gamma_{l}=\left\{x=(y, z) \in \Gamma: 2^{l-1}<r<2^{l+1}\right\}, l=0, \pm 1, \pm 2, \ldots$ Let $\xi_{l}$ be the same functions as in the proof of Lemma 7.1 .

Similarly to (7.8), we have

$$
\left\|\left.u\right|_{\Gamma}\right\|_{H_{a}^{0}(\Gamma)}^{2} \approx \sum_{l=-\infty}^{+\infty}\left\|\left.\left(\xi_{l} u\right)\right|_{\Gamma_{l}}\right\|_{H_{a}^{0}\left(\Gamma_{l}\right)}^{2}
$$

where the symbol $\approx$ means the equivalence of the norms.

Using the theorem on the extension of functions from a domain with Lipschitz boundary to $\mathbb{R}^{n}$ and applying the interpolation inequality for the Sobolev space $W^{1}\left(\mathbb{R}^{n}\right)$ (see 2 , Sec. 1]), we obtain

$$
q\left\|\left.v\right|_{\Gamma_{0}}\right\|_{L_{2}\left(\Theta_{0}\right)}^{2} \leq k_{1}\left(\|v\|_{W^{1}\left(\Theta_{0}\right)}^{2}+q^{2}\|v\|_{L_{2}\left(\Theta_{0}\right)}^{2}\right),
$$

for all $v \in W^{k}\left(\Theta_{0}\right)$ and $q>0$, where $k_{1}>0$ does not depend on $v$ or $q$.

We introduce the new variables $x^{\prime}=2^{-l} x$. Using the equivalence of the norms (7.12) and the interpolation inequality (7.13) with $q=|p| 2^{l}$ and reverting to the variables 
$x=2^{l} x^{\prime}$, we have

$$
\begin{aligned}
& |p| \cdot\left\|\left.u\right|_{\Gamma}\right\|_{H_{a}^{0}(\Gamma)}^{2} \leq\left. k_{2}|p| \sum_{l=-\infty}^{+\infty} 2^{(2 a+n-1) l} \int_{\Gamma_{0}}\left|\left(\xi_{l} u\right)\left(x^{\prime}\right)\right|_{\Gamma_{0}}\right|^{2} d \Gamma_{0} \\
& \quad \leq k_{3} \sum_{l=-\infty}^{+\infty} 2^{(2 a+n-2) l}\left\{\sum_{|\alpha| \leq 1} \int_{\Theta_{0}}\left|D_{x^{\prime}}^{\alpha}\left(\xi_{l} u\right)\left(x^{\prime}\right)\right|^{2} d x^{\prime}+|p|^{2} 2^{2 l} \int_{\Theta_{0}}\left|\left(\xi_{l} u\right)\left(x^{\prime}\right)\right|^{2} d x^{\prime}\right\} \\
& \quad \leq k_{4} \sum_{l=-\infty}^{+\infty}\left(\left\|\xi_{l} u\right\|_{H_{a}^{1}\left(\Theta_{l}\right)}^{2}+|p|^{2}\left\|\xi_{l} u\right\|_{H_{a}^{0}\left(\Theta_{l}\right)}^{2}\right),
\end{aligned}
$$

where $k_{2}, k_{3}, k_{4}>0$ do not depend on $u$ and $p$. Combining this inequality with (7.8) yields (7.10).

In particular, it follows from Lemmas 7.1 and 7.2 that

$$
\begin{aligned}
\left\|\left.u\right|_{\Gamma}\right\|_{V_{a}^{k-1 / 2}(\Gamma)} & \leq c\|u\|_{V_{a}^{k}(\Theta)}, \\
\left\|\left.u\right|_{\Gamma_{i}}\right\|_{H_{a}^{k-1 / 2}\left(\Gamma_{i}\right)} & \leq C\|u\|_{H_{a}^{k}(Q)},
\end{aligned}
$$

where $c, C>0$ do not depend on $u$ and $p$.

Lemma 7.3. For all $\psi \in V_{a}^{k-1 / 2}(\Gamma), k \geq 2$ is an integer, $p \in \mathbb{R}^{d}$, and integer $s$, $0<s<k$, we have

$$
|p|^{k-s-1 / 2}\|\psi\|_{H_{a}^{s}(\Gamma)} \leq c\|\psi\|_{V_{a}^{k-1 / 2}(\Gamma)},
$$

where $\Gamma$ is defined by (7.2) and $c>0$ does not depend on $\psi$ or $p$.

Proof. The proof is based on the following interpolation inequality for Sobolev spaces in $\mathbb{R}^{n-1}$ (see [2, Sec. 1]):

$$
q^{2(k-s-1 / 2)}\|v\|_{W^{s}\left(\mathbb{R}^{n-1}\right)}^{2} \leq k_{1}\left(\|v\|_{W^{k-1 / 2}\left(\mathbb{R}^{n-1}\right)}^{2}+q^{2(k-1 / 2)}\|v\|_{L_{2}\left(\mathbb{R}^{n-1}\right)}^{2}\right)
$$

for all $v \in W^{k-1 / 2}\left(\mathbb{R}^{n-1}\right), q>0$, and integer $s, 0<s<k$, where $k_{1}>0$ does not depend on $v$ or $q$. We will use the following equivalent norm in the space $W^{k-1 / 2}\left(\mathbb{R}^{n-1}\right)$ :

$$
\left(\sum_{|\alpha|=k-1} \int_{\mathbb{R}^{n-1}} \int_{\mathbb{R}^{n-1}}\left|D^{\alpha} v\left(x_{1}\right)-D^{\alpha} v\left(x_{2}\right)\right|^{2} \frac{d x_{1} d x_{2}}{\left|x_{1}-x_{2}\right|^{n}}+\sum_{|\alpha| \leq k-1} \int_{\mathbb{R}^{n-1}}\left|D^{\alpha} v(x)\right|^{2} d x\right)^{1 / 2}
$$

(see, e.g., 30]).

Denote $\Gamma_{l}=\left\{x=(y, z) \in \Gamma: 2^{l-1}<r<2^{l+1}\right\}, l=0, \pm 1, \pm 2, \ldots$. Let $\xi_{l}$ be the same functions as in the proofs of Lemmas 7.1 and 7.2

Using relations (7.7) and the fact that $\operatorname{supp} \xi_{l} \cap \overline{\Theta_{j}}=\varnothing$ for $j \neq l-1, l, l+1$, one can easily verify that

$$
\|\psi\|_{H_{a}^{s}(\Gamma)} \approx\left(\sum_{l=-\infty}^{+\infty}\left\|\xi_{l} \psi\right\|_{H_{a}^{s}\left(\Gamma_{l}\right)}^{2}\right)^{1 / 2}
$$

for $s=0,1 / 2,1,3 / 2, \ldots$, where the symbol $\approx$ means the equivalence of the norms (in particular, see Lemma 1.1 in [20] for noninteger $s$ ).

Further, introducing the new variables $x^{\prime}=2^{-l} x$ and using equivalence (7.19), the interpolation inequality (7.17) with $q=|p| 2^{l}$, and the equivalent norm (7.18) in the 
Sobolev space (which is possible because the functions $\xi_{l}$ are compactly supported), we obtain

$$
\begin{aligned}
|p|^{2(k-s-1 / 2)}\|\psi\|_{H_{a}^{s}(\Gamma)}^{2} & \\
\leq & k_{2} \sum_{l=-\infty}^{+\infty} 2^{2 l(a-k+1 / 2)} 2^{l(n-1)} \cdot|p|^{2(k-s-1 / 2)} 2^{2 l(k-s-1 / 2)} \sum_{|\alpha| \leq s} \int_{\Gamma_{0}}\left|D_{x^{\prime}}^{\alpha}\left(\xi_{l} \psi\right)\left(x^{\prime}\right)\right|^{2} d x^{\prime} \\
\leq & k_{3} \sum_{l=-\infty}^{+\infty} 2^{2 l(a-k+1 / 2)} 2^{l(n-1)} \\
& \times \sum_{|\alpha|=k-1} \int_{\Gamma} \int_{\Gamma}\left|D_{x^{\prime}}^{\alpha}\left(\xi_{l} \psi\right)\left(x_{1}^{\prime}\right)-D_{x^{\prime}}^{\alpha}\left(\xi_{l} \psi\right)\left(x_{2}^{\prime}\right)\right|^{2} \frac{d x_{1}^{\prime} d x_{2}^{\prime}}{\left|x_{1}^{\prime}-x_{2}^{\prime}\right|^{n}} \\
& \left.+\sum_{|\alpha| \leq k-1} \int_{\Gamma_{0}}\left|D_{x^{\prime}}^{\alpha}\left(\xi_{l} \psi\right)\left(x^{\prime}\right)\right|^{2} d x^{\prime}+|p|^{2(k-1 / 2)} 2^{2 l(k-1 / 2)} \int_{\Gamma_{0}}\left|\left(\xi_{l} \psi\right)\left(x^{\prime}\right)\right|^{2} d x^{\prime}\right) \\
\leq & k_{4} \sum_{l=-\infty}^{+\infty} 2^{2 l(a-k+1 / 2)} 2^{l(n-1)} \\
\times & \left(\left.\sum_{|\alpha|=k-1} \int_{\Gamma} \int_{\Gamma}|| y_{1}^{\prime}\right|^{a} D_{x^{\prime}}^{\alpha}\left(\xi_{l} \psi\right)\left(x_{1}^{\prime}\right)-\left.\left|y_{2}^{\prime}\right|^{a} D_{x^{\prime}}^{\alpha}\left(\xi_{l} \psi\right)\left(x_{2}^{\prime}\right)\right|^{2} \frac{d x_{1}^{\prime} d x_{2}^{\prime}}{\left|x_{1}^{\prime}-x_{2}^{\prime}\right|^{n}}\right. \\
& +\sum_{|\alpha| \leq k-1} \int_{\Gamma_{0}}\left|y^{\prime}\right|^{2(a+|\alpha|-k+1 / 2)}\left|D_{x^{\prime}}^{\alpha}\left(\xi_{l} \psi\right)\left(x^{\prime}\right)\right|^{2} d x^{\prime} \\
& \left.+|p|^{2(k-1 / 2)} 2^{2 l(k-1 / 2)} \int_{\Gamma_{0}}\left|y^{\prime}\right|^{2 a}\left|\left(\xi_{l} \psi\right)\left(x^{\prime}\right)\right|^{2} d x^{\prime}\right),
\end{aligned}
$$

where $k_{2}, k_{3}, \ldots>0$ do not depend on $\psi$ and $p, x_{i}^{\prime}=\left(y_{i}^{\prime}, z_{i}^{\prime}\right) \in \Gamma, y_{i}^{\prime} \in \mathbb{R}^{2}$, and $z_{i}^{\prime} \in \mathbb{R}^{n-2}$, $i=1,2$. In the last inequality, we have also used the fact that $\operatorname{supp} \xi_{l} \cap \overline{Q_{j}}=\varnothing$ for $j \neq l-1, l, l+1$. Reverting to the variables $x=2^{l} x^{\prime}$, we have

$$
\begin{aligned}
& |p|^{2(k-s-1 / 2)}\|\psi\|_{H_{a}^{s}(\Gamma)}^{2} \\
& \quad \leq k_{5} \sum_{l=-\infty}^{+\infty}\left(\left.\sum_{|\alpha|=k-1} \int_{\Gamma} \int_{\Gamma}|| y_{1}\right|^{a} D^{\alpha}\left(\xi_{l} \psi\right)\left(x_{1}\right)-\left.\left|y_{2}\right|^{a} D^{\alpha}\left(\xi_{l} \psi\right)\left(x_{2}\right)\right|^{2} \frac{d x_{1} d x_{2}}{\left|x_{1}-x_{2}\right|^{n}}\right. \\
& \left.\quad+\sum_{|\alpha| \leq k-1} \int_{\Gamma_{l}}|y|^{2(a+|\alpha|-k+1 / 2)}\left|D^{\alpha}\left(\xi_{l} \psi\right)(x)\right|^{2} d x+|p|^{2(k-1 / 2)} \int_{\Gamma_{l}}|y|^{2 a}\left|\left(\xi_{l} \psi\right)(x)\right|^{2} d x\right),
\end{aligned}
$$

where $x_{i}=\left(y_{i}, z_{i}\right) \in \Gamma, y_{i} \in \mathbb{R}^{2}$, and $z_{i} \in \mathbb{R}^{n-2}, i=1,2$. It follows from this inequality and from Lemma 1.3 in 20] (on the equivalent norms in the weighted trace spaces) that

$$
|p|^{2(k-s-1 / 2)}\|\psi\|_{H_{a}^{s}(\Gamma)}^{2} \leq k_{6} \sum_{l=-\infty}^{+\infty}\left(\left\|\xi_{l} \psi\right\|_{H_{a}^{k-1 / 2}\left(\Gamma_{l}\right)}^{2}+|p|^{2(k-1 / 2)}\left\|\xi_{l} \psi\right\|_{H_{a}^{0}\left(\Gamma_{l}\right)}^{2}\right) .
$$

Combining (7.20) with the equivalence of the norms (7.19), we obtain (7.16). 
7.2. Consider the differential operators

$$
\begin{aligned}
A^{0}(p) \equiv A^{0}(x, D, p) & =\sum_{|\alpha|+|\beta|=2 m} a_{\alpha \beta}(x) p^{\beta} D^{\alpha}, \\
B_{i \mu s}^{0}(p) \equiv B_{i \mu s}^{0}(x, D, p) & =\sum_{|\alpha|+|\beta|=m_{i \mu}} b_{i \mu s \alpha \beta}(x) p^{\beta} D^{\alpha},
\end{aligned}
$$

where $a_{\alpha \beta}, b_{i \mu s \alpha \beta} \in C^{\infty}\left(\mathbb{R}^{n}\right)$ are complex-valued functions $\left(i=1, \ldots, N_{0} ; \mu=1, \ldots, m\right.$; $\left.s=0, \ldots, S_{i}\right), \beta=\left(\beta_{1}, \ldots, \beta_{d}\right),|\beta|=\left|\beta_{1}\right|+\cdots+\left|\beta_{d}\right|, p^{\beta}=p_{1}^{\beta_{1}} \ldots p_{d}^{\beta_{d}}$, and $m_{i \mu} \leq 2 m-1$.

We study the following nonlocal elliptic problem:

$$
\begin{gathered}
A(p) u \equiv A^{0}(p) u+A^{1}(p) u=f_{0}(x), \quad x \in Q, \\
B_{i \mu}(p) u \equiv \sum_{j=0}^{3} B_{i \mu}^{j}(p) u=f_{i \mu}(x), \quad x \in \Gamma_{i} ; i=1, \ldots, N_{0} ; \mu=1, \ldots, m .
\end{gathered}
$$

Here

$$
B_{i \mu}^{0}(p) u=\left.B_{i \mu 0}^{0}(p) u\right|_{\Gamma_{i}}, \quad B_{i \mu}^{1}(p) u=\left.\sum_{s=1}^{S_{i}}\left(B_{i \mu s}^{0}(x, D, p)(\xi u)\right)\left(\omega_{i s}(x)\right)\right|_{\Gamma_{i}},
$$

the function $\xi$ and the transformations $\omega_{i s}$ are the same as in Sec. 6. In particular, we assume that Conditions 1.3 and 1.4 hold.

Introduce the variable $t=\left(t_{1}, \ldots, t_{d}\right) \in \mathbb{R}^{d}$ and formally replace the expressions $p^{\beta}$ in the operators $A^{0}\left(x, D_{x}, p\right)$ and $B_{i \mu 0}^{0}\left(x, D_{x}, p\right)$ by the differential operators $D_{t}^{\beta}$. Assume that the following conditions hold (cf. [2, 20]).

Condition 7.1. The operator $A^{0}\left(x, D_{x}, D_{t}\right)$ is properly elliptic for $(x, t) \in \bar{Q} \times \mathbb{R}^{d}$.

Condition 7.2. The system $\left\{B_{i \mu 0}^{0}\left(x, D_{x}, D_{t}\right)\right\}_{\mu=1}^{m}$ covers the operator $A^{0}\left(x, D_{x}, D_{t}\right)$ and is normal for all $i=1, \ldots, N_{0}$ and $(x, t) \in \overline{\Gamma_{i}} \times \mathbb{R}^{d}$.

We also assume that the following conditions for the operators $A^{1}(p), B_{i \mu}^{2}(p)$, and $B_{i \mu}^{3}(p)$ hold.

Condition 7.3 (Smallness of perturbations). We have

$$
\begin{gathered}
\left\|A^{1}(p) u\right\|_{H_{a}^{l}(Q)} \leq c_{1}\|u\|_{H_{a}^{l+2 m-1}(Q)}, \\
\left\|B_{i \mu}^{3}(p) u\right\|_{H_{a}^{l+2 m-m_{i \mu}-1 / 2}\left(\Gamma_{i}\right)} \leq c_{2}\|u\|_{H_{a}^{l+2 m-1}(Q)},
\end{gathered}
$$

where $i=1, \ldots, N_{0}, \mu=1, \ldots, m$, and $c_{1}, c_{2}>0$ do not depend on $u$ or $p$.

Condition 7.4 (Separability from the conjugation points). There exist numbers $\sigma>0$ and $\varkappa_{1}>\varkappa_{2}>0$ such that

$$
\left\|B_{i \mu}^{2}(p) u\right\|_{H_{a}^{l+2 m-m_{i \mu}-1 / 2}\left(\Gamma_{i}\right)} \leq c_{1}\|u\|_{H_{a}^{l+2 m}\left(Q \backslash \overline{\mathcal{K}_{1}^{\varkappa_{1}}}\right)}
$$

for all $u \in H_{a}^{l+2 m}\left(Q \backslash \overline{\mathcal{K}_{1}^{\varkappa_{1}}}\right)$ and

$$
\left\|B_{i \mu}^{2}(p) u\right\|_{H_{a}^{l+2 m-m_{i \mu}-1 / 2}\left(\Gamma_{i} \backslash \overline{\mathcal{K}_{1}^{\varkappa 2}}\right)} \leq c_{2}\|u\|_{H_{a}^{l+2 m}\left(Q_{\sigma}\right)}
$$

for all $u \in H_{a}^{l+2 m}\left(Q_{\sigma}\right)$; here $i=1, \ldots, N_{0} ; \mu=1, \ldots, m ; c_{1}, c_{2}>0$ do not depend on $u$ or $p$.

Remark 7.1. 1. It follows from Condition 7.3 and from Lemma 7.1 that

$$
\begin{gathered}
\left\|A^{1}(p) u\right\|_{H_{a}^{l}(Q)} \leq c_{1}|p|^{-1}\|u\|_{H_{a}^{l+2 m}(Q)}, \\
\left\|B_{i \mu}^{3}(p) u\right\|_{H_{a}^{l+2 m-m_{i \mu}-1 / 2}\left(\Gamma_{i}\right)} \leq c_{2}|p|^{-1}\|u\|_{H_{a}^{l+2 m}(Q)} .
\end{gathered}
$$


Therefore, the norms of the operators $A^{1}(p)$ and $B_{i \mu}^{3}(p)$ are small, provided that $|p|$ is large.

2. Condition 7.4 is an analog of Condition 6.2.

Remark 7.2. Let the transformations $\omega_{i s}$ and the set $K$ be the same as in Secs. 15 , Consider the problem

$$
\begin{gathered}
\sum_{|\alpha|+|\beta| \leq 2 m} a_{\alpha \beta}(x) p^{\beta} D^{\alpha} u(x)=f_{0}(x), \quad x \in Q, \\
\left.\sum_{|\alpha|+|\beta| \leq m_{i \mu}} \sum_{s=0}^{S_{i}} b_{i \mu s \alpha \beta}\left(\omega_{i s}(x)\right) p^{\beta}\left(D^{\alpha} u\right)\left(\omega_{i s}(x)\right)\right|_{\Gamma_{i}}=f_{i \mu}(x), \\
x \in \Gamma_{i} ; i=1, \ldots, N_{0} ; \mu=1, \ldots, m .
\end{gathered}
$$

This problem can be represented in the form (7.21), (7.22). Indeed, set

$$
\begin{aligned}
A^{0}(p) & =\sum_{|\alpha|+|\beta|=2 m} a_{\alpha \beta}(x) p^{\beta} D^{\alpha}, \quad A^{1}(p)=\sum_{|\alpha|+|\beta| \leq 2 m-1} a_{\alpha \beta}(x) p^{\beta} D^{\alpha}, \\
B_{i \mu}^{0}(p) u & =\left.\sum_{|\alpha|+|\beta|=m_{i \mu}} b_{i \mu 0 \alpha \beta}(x) p^{\beta} D^{\alpha} u\right|_{\Gamma_{i}}, \\
B_{i \mu}^{1}(p) u & =\left.\sum_{|\alpha|+|\beta|=m_{i \mu}} \sum_{s=1}^{S_{i}} b_{i \mu s \alpha \beta}\left(\omega_{i s}(x)\right) p^{\beta}\left(D^{\alpha}(\xi u)\right)\left(\omega_{i s}(x)\right)\right|_{\Gamma_{i}}, \\
B_{i \mu}^{2}(p) u & =\left.\sum_{|\alpha|+|\beta|=m_{i \mu}} \sum_{s=1}^{S_{i}} b_{i \mu s \alpha \beta}\left(\omega_{i s}(x)\right) p^{\beta}\left(D^{\alpha}((1-\xi) u)\right)\left(\omega_{i s}(x)\right)\right|_{\Gamma_{i}}, \\
B_{i \mu}^{3}(p) u & =\left.\sum_{|\alpha|+|\beta| \leq m_{i \mu}-1} \sum_{s=0}^{S_{i}} b_{i \mu s \alpha \beta}\left(\omega_{i s}(x)\right) p^{\beta}\left(D^{\alpha} u\right)\left(\omega_{i s}(x)\right)\right|_{\Gamma_{i}} .
\end{aligned}
$$

Clearly, the operator $A^{1}(p)$ satisfies Condition 7.3 .

Let $s=0, \ldots, S_{i}$ and $|\alpha|+|\beta| \leq m_{i \mu}-1$. Denote by $u_{1}$ an extension of the function $u$ to $Q \cup \omega_{i s}\left(\Omega_{i}\right)$, defined by Lemma 4.4 and satisfying the inequalities

$$
\left\|u_{1}\right\|_{H_{a}^{\nu}\left(Q \cup \omega_{i s}\left(\Omega_{i}\right)\right)} \leq k_{1}\|u\|_{H_{a}^{\nu}(Q)}, \quad \nu=0, \ldots, l+2 m-1 .
$$

Clearly,

$$
\left.b_{i \mu s \alpha \beta}\left(\omega_{i s}(x)\right) p^{\beta}\left(D^{\alpha} u\right)\left(\omega_{i s}(x)\right)\right|_{\Gamma_{i}}=\left.b_{i \mu s \alpha \beta}\left(\omega_{i s}(x)\right) p^{\beta}\left(D^{\alpha} u_{1}\right)\left(\omega_{i s}(x)\right)\right|_{\Gamma_{i}} .
$$

Therefore, using (7.15) and (7.25), we have

$$
\begin{gathered}
\left\|\left.b_{i \mu s \alpha \beta}\left(\omega_{i s}(x)\right) p^{\beta}\left(D^{\alpha} u\right)\left(\omega_{i s}(x)\right)\right|_{\Gamma_{i}}\right\|_{H_{a}^{l+2 m-m_{i \mu}-1 / 2}\left(\Gamma_{i}\right)} \\
\leq k_{2}\left\|u_{1}\right\|_{H_{a}^{l+2 m-1}\left(\omega_{i s}\left(\Omega_{i}\right)\right)} \leq k_{3}\|u\|_{H_{a}^{l+2 m-1}(Q)}
\end{gathered}
$$

for $\alpha$ and $\beta$ such that $|\alpha|+|\beta| \leq m_{i \mu}-1$. This inequality proves that the operators $B_{i \mu}^{3}(p)$ satisfy Condition 7.3 .

To show that the operators $B_{i \mu}^{2}(p)$ satisfy Condition 7.4 , one must repeat the proof of Lemma 4.6 with the norms $\|\cdot\|$ replaced by the norms $\|\cdot\|$, taking into account inequality (7.15).

We consider the linear bounded operators $\mathbf{L}^{0}(p), \mathbf{L}^{1}(p), \mathbf{L}(p): H_{a}^{l+2 m}(Q) \rightarrow \mathcal{H}_{a}^{l}(Q, \Gamma)$ given by

$$
\mathbf{L}^{0}(p)=\left\{A(p), B_{i \mu}^{0}(p)\right\}, \quad \mathbf{L}^{1}(p)=\left\{A(p), B_{i \mu}^{0}(p)+B_{i \mu}^{1}(p)\right\}, \quad \mathbf{L}(p)=\left\{A(p), B_{i \mu}(p)\right\} .
$$


The invertibility of the operator $\mathbf{L}^{0}(p)$ was proved in $[20$. Our aim is to study the operator $\mathbf{L}^{1}(p)$ and then $\mathbf{L}(p)$. First, we will consider model problems with a parameter corresponding to the points of the sets $\mathcal{K}_{1}$ and $K_{2}$.

7.3. Fix a point $g \in \mathcal{K}_{1}$. Using the reasoning similar to that in Sec. 1, we arrive at the following model problem (cf. (1.9), (1.10)):

$$
\begin{array}{r}
A_{j}\left(x, D_{y}, D_{z}, p\right) v_{j}(x)=f_{j}(x), \quad x \in \Theta_{j} ; j=1, \ldots, N, \\
\left.\sum_{k=1}^{N} \sum_{s=0}^{S_{j \rho k}}\left(B_{j \rho \mu k s}\left(x, D_{y}, D_{z}, p\right) v_{k}\right)\left(\mathcal{G}_{j \rho k s} y, z\right)\right|_{\Gamma_{j \rho}}=f_{j \rho \mu}(x), \quad x \in \Gamma_{j \rho} ; \\
j=1, \ldots, N ; \rho=1,2 ; \mu=1, \ldots, m,
\end{array}
$$

where $A_{j}\left(x, D_{y}, D_{z}, p\right)$ and $B_{j \rho \mu k s}\left(x, D_{y}, D_{z}, p\right)$ are differential operators of order $2 m$ and $m_{j \rho \mu}$, respectively, with the parameter $p$, having variable coefficients of class $C^{\infty}$, while $\Theta_{j}, \Gamma_{j \rho}$, and $\mathcal{G}_{j \rho k s}$ are the same as in (1.9), (1.10).

Introduce the spaces of vector-valued functions

$$
\mathcal{V}_{a}^{k}(\Theta)=\prod_{j=1}^{N} V_{a}^{k}\left(\Theta_{j}\right), \quad \mathcal{V}_{a}^{l}(\Theta, \Gamma)=\mathcal{V}_{a}^{l}(\Theta) \times \prod_{j=1}^{N} \prod_{\rho=1,2} \prod_{\mu=1}^{m} V_{a}^{l+2 m-m_{j \rho \mu}-1 / 2}\left(\Gamma_{j \rho}\right),
$$

where $m_{j \rho \mu}$ is the order of the operator $B_{j \rho \mu k s}\left(x, D_{y}, D_{z}, p\right)$. Consider the linear bounded operator $\mathcal{L}_{g}(p): \mathcal{V}_{a}^{l+2 m}(\Theta) \rightarrow \mathcal{V}_{a}^{l}(\Theta, \Gamma)$ given by

$$
\mathcal{L}_{g}(p) v=\left\{A_{j}\left(D_{y}, D_{z}, p\right) v_{j}(y, z),\left.\sum_{k, s}\left(B_{j \rho \mu k s}\left(D_{y}, D_{z}, p\right) v_{k}\right)\left(\mathcal{G}_{j \rho k s} y, z\right)\right|_{\Gamma_{j \rho}}\right\},
$$

where $A_{j}\left(D_{y}, D_{z}, p\right)$ and $B_{j \rho \mu k s}\left(D_{y}, D_{z}, p\right)$ are principal homogeneous part: 3 of the operators $A_{j}\left(0, D_{y}, D_{z}, p\right)$ and $B_{j \rho \mu k s}\left(0, D_{y}, D_{z}, p\right)$, respectively. Clearly, if we replace $p^{\beta}$ by $D_{t}^{\beta}$, then each of the obtained operators $A_{j}\left(D_{y}, D_{z}, D_{t}\right)$ will be properly elliptic for $(x, t) \in \overline{\Theta_{j}} \times \mathbb{R}^{d}$, while the system $\left\{B_{j \rho \mu j 0}\left(D_{y}, D_{z}, D_{t}\right)\right\}_{\mu=1}^{m}$ will cover the operator $A_{j}\left(D_{y}, D_{z}, D_{t}\right)$ and be normal for all $(x, t) \in \Gamma_{j \rho} \times \mathbb{R}^{d}, j=1, \ldots, N$, and $\rho=1,2$.

We also set

$$
\mathcal{L}_{g}^{\prime}(p) v=\left\{A_{j}^{0}\left(x, D_{y}, D_{z}, p\right) v_{j}(y, z),\left.\sum_{k, s}\left(B_{j \rho \mu k s}^{0}\left(x, D_{y}, D_{z}, p\right) v_{k}\right)\left(\mathcal{G}_{j \rho k s} y, z\right)\right|_{\Gamma_{j \rho}}\right\},
$$

where $A_{j}^{0}\left(x, D_{y}, D_{z}, p\right)$ and $B_{j \rho \mu k s}^{0}\left(x, D_{y}, D_{z}, p\right)$ are principal homogeneous parts of the operators $A_{j}\left(x, D_{y}, D_{z}, p\right)$ and $B_{j \rho \mu k s}\left(x, D_{y}, D_{z}, p\right)$, respectively.

Further, we set

$$
\mathcal{L}_{g}(\eta, p) V=\left\{A_{j}\left(D_{y}, \eta, p\right) V_{j}(y),\left.\sum_{k, s}\left(B_{j \rho \mu k s}\left(D_{y}, \eta, p\right) V_{k}\right)\left(\mathcal{G}_{j \rho k s} y\right)\right|_{\Gamma_{j \rho}}\right\}, \quad \eta \in \mathbb{R}^{n-2} .
$$

Replacing $(\eta, p)$ by $\omega=(\eta, p) /|(\eta, p)|$, we obtain the bounded operator

$$
\mathcal{L}_{g}(\omega): \mathcal{E}_{a}^{l+2 m}(\theta) \rightarrow \mathcal{E}_{a}^{l}(\theta, \gamma)
$$

given by

$$
\mathcal{L}_{g}(\omega) V=\left\{A_{j}\left(D_{y}, \omega\right) V_{j}(y),\left.\sum_{k, s}\left(B_{j \rho \mu k s}\left(D_{y}, \omega\right) V_{k}\right)\left(\mathcal{G}_{j \rho k s} y\right)\right|_{\Gamma_{j \rho}}\right\}, \quad \omega \in S^{n+d-3}
$$

Finally, we consider the analytic operator-valued function

$$
\hat{\mathcal{L}}_{g}(\lambda): \mathcal{W}^{l+2 m}\left(d_{1}, d_{2}\right) \rightarrow \mathcal{W}^{l}\left[d_{1}, d_{2}\right]
$$

given by (2.2).

\footnotetext{
3In this section, the notion "principal homogeneous part" takes into account the parameter $p$, e.g., the operator $A_{j}\left(D_{y}, D_{z}, p\right)$ consists of the terms $a_{j \alpha \beta}(x) p^{\beta} D^{\alpha}$, where $|\alpha|+|\beta|=2 m$.
} 
In this subsection, we prove that the absence of eigenvalues of $\hat{\mathcal{L}}_{g}(\lambda)$ on the line $\operatorname{Im} \lambda=a+1-l-2 m$ and the triviality of the kernel and cokernel of $\mathcal{L}_{g}(\omega)$ guarantee the existence of the inverse operators $\mathcal{L}_{g}^{-1}(p)$ for $p \in \mathbb{R}^{d} \backslash\{0\}$, uniformly bounded in the corresponding norms $\|\cdot\|$. We introduce these norms by setting

$$
\begin{gathered}
\|u\|_{\mathcal{V}_{a}^{k}(\Theta)}=\left(\sum_{j}\|u\|_{V_{a}^{k}\left(\Theta_{j}\right)}^{2}\right)^{1 / 2}, \\
\|f\|_{\mathcal{V}_{a}^{l}(\Theta, \Gamma)}=\left(\sum_{j}\left\|f_{j}\right\|_{V_{a}^{l}\left(\Theta_{j}\right)}^{2}+\sum_{j, \rho, \mu}\left\|f_{j \rho \mu}\right\|_{V_{a}^{l+2 m-m_{j \rho \mu}-1 / 2}\left(\Gamma_{j}\right)}^{2}\right)^{1 / 2}, \quad f=\left\{f_{j}, f_{j \rho \mu}\right\} .
\end{gathered}
$$

Theorem 7.1. Let Conditions 7.1, 7.2, 1.3, and 1.4 hold. Assume that the line $\operatorname{Im} \lambda=$ $a+1-l-2 m$ contains no eigenvalues of $\hat{\mathcal{L}}_{g}(\lambda)$ and $\operatorname{dim} \mathcal{N}\left(\mathcal{L}_{g}(\omega)\right)=\operatorname{codim} \mathcal{R}\left(\mathcal{L}_{g}(\omega)\right)=0$ for any $\omega \in S^{n+d-3}$. Then the operator $\mathcal{L}_{g}(p)$ is an isomorphism for $p \in \mathbb{R}^{d} \backslash\{0\}$ and

$$
\|u\|_{\mathcal{V}_{a}^{l+2 m}(\Theta)} \leq c\left\|\mathcal{L}_{g}(p) u\right\|_{\mathcal{V}_{a}^{l}(\Theta, \Gamma)}, \quad p \in \mathbb{R}^{d} \backslash\{0\}
$$

where $c>0$ does not depend on $u$ or $p$.

To prove Theorem 7.1 we preliminarily consider the bounded operator

$$
\mathcal{L}_{g}(p): \mathcal{E}_{a}^{l+2 m}(\Theta) \rightarrow \mathcal{E}_{a}^{l}(\Theta, \Gamma)
$$

given by (7.28) for $p \in S^{d-1}$, where

$$
\mathcal{E}_{a}^{k}(\Theta)=\prod_{j=1}^{N} E_{a}^{k}\left(\Theta_{j}\right), \quad \mathcal{E}_{a}^{l}(\Theta, \Gamma)=\mathcal{E}_{a}^{l}(\Theta) \times \prod_{j=1}^{N} \prod_{\rho=1,2} \prod_{\mu=1}^{m} E_{a}^{l+2 m-m_{j \rho \mu}-1 / 2}\left(\Gamma_{j \rho}\right),
$$

while $E_{a}^{k}\left(\Theta_{j}\right)$ is the completion of the set $C_{0}^{\infty}\left(\overline{\Theta_{j}} \backslash\{0\}\right)$ with respect to the norm

$$
\|v\|_{E_{a}^{k}\left(\Theta_{j}\right)}=\left(\sum_{|\alpha| \leq k} \int_{\Theta_{j}}|y|^{2 a}\left(|y|^{2(|\alpha|-k)}+1\right)\left|D_{x}^{\alpha} v(x)\right|^{2} d x\right)^{1 / 2}
$$

and $E_{a}^{k-1 / 2}\left(\Gamma_{j \rho}\right)(k \geq 1$ is an integer $)$ is the space of traces on $\Gamma_{j \rho}$ with the norm

$$
\|\psi\|_{E_{a}^{k-1 / 2}\left(\Gamma_{j \rho}\right)}=\inf \|v\|_{E_{a}^{k}\left(\Theta_{j}\right)}\left(v \in E_{a}^{k}\left(\Theta_{j}\right):\left.v\right|_{\Gamma_{j \rho}}=\psi\right) .
$$

Lemma 7.4. Let Conditions 7.2, 1.3, and 1.4 hold, and let $f_{j \rho \mu} \in E_{a}^{l+2 m-m_{j \rho \mu}-1 / 2}\left(\Gamma_{j \rho}\right)$. Then there exists a function $u \in \mathcal{E}_{a}^{l+2 m}(\Theta)$ such that

$$
\begin{array}{ll}
\left.\sum_{k, s}\left(B_{j \rho \mu k s}\left(D_{y}, D_{z}, p\right) u_{k}\right)\left(\mathcal{G}_{j \rho k s} y, z\right)\right|_{\Gamma_{j \rho}}=f_{j \rho \mu}, & p \in S^{d-1} \\
\|u\|_{\mathcal{E}_{a}^{l+2 m}(\Theta)} \leq c \sum_{j, \rho, \mu}\left\|f_{j \rho \mu}\right\|_{E_{a}^{l+2 m-m_{j \rho \mu}-1 / 2}\left(\Gamma_{j \rho}\right)}, & p \in S^{d-1}
\end{array}
$$

where $c>0$ does not depend on $u$ or $p$.

Proof. By Lemma 9.2' in [20], there exists a function $v=\left(v_{1}, \ldots, v_{N}\right) \in \mathcal{E}_{a}^{l+2 m}(\Theta)$ such that $\left.B_{j \rho \mu j 0}\left(D_{y}, D_{z}, p\right) v_{j}\right|_{\Gamma_{j \rho}}=f_{j \rho \mu}$ and

$$
\|v\|_{\mathcal{E}_{a}^{l+2 m}(\Theta)} \leq k_{1} \sum_{j, \rho, \mu}\left\|f_{j \rho \mu}\right\|_{E_{a}^{l+2 m-m_{j \rho \mu}-1 / 2}\left(\Gamma_{j \rho}\right)}, \quad p \in S^{d-1} .
$$

Let $d_{0}$ be the number defined in (5.21). Consider functions $\xi_{k} \in C^{\infty}(\mathbb{R})$ depending on the polar angle $\varphi$ of the point $y \in \mathbb{R}^{2}$, such that $\xi_{k}(\varphi)=1$ for $d_{k 1} \leq \varphi \leq d_{k 1}+d_{0} / 2$ and $d_{k 2}-d_{0} / 2 \leq \varphi \leq d_{k 2}$ and $\xi_{k}(\varphi)=0$ for $d_{k 1}+d_{0} \leq \varphi \leq d_{k 2}-d_{0}$.

Since the operator of multiplication by $\xi_{j}$ is bounded in $E_{a}^{l+2 m}\left(\Theta_{j}\right)$, we see that the function $u=\left(\xi_{1} v_{1}, \ldots, \xi_{N} v_{N}\right)$ is the desired one. 
Lemma 7.5. Let the conditions of Theorem 7.1 be fulfilled. Then the operator $\mathcal{L}_{g}(p)$ : $\mathcal{E}_{a}^{l+2 m}(\Theta) \rightarrow \mathcal{E}_{a}^{l}(\Theta, \Gamma)$ is an isomorphism for $p \in S^{d-1}$ and

$$
\|u\|_{\mathcal{E}_{a}^{l+2 m}(\Theta)} \leq c\left\|\mathcal{L}_{g}(p) u\right\|_{\mathcal{E}_{a}^{l}(\Theta, \Gamma)}, \quad p \in S^{d-1},
$$

where $c>0$ does not depend on $u$ or $p$.

Proof. 1. Due to Lemma 7.4 it suffices to prove the unique solvability of the problem

$$
\begin{gathered}
A_{j}\left(D_{y}, D_{z}, p\right) u_{j}(y, z)=f_{j}(y, z), \quad(y, z) \in \Theta_{j}, \\
\left.\sum_{k, s}\left(B_{j \rho \mu k s}\left(D_{y}, D_{z}, p\right) u_{k}\right)\left(\mathcal{G}_{j \rho k s} y, z\right)\right|_{\Gamma_{j \rho}}=0
\end{gathered}
$$

and to show that

$$
\|u\|_{\mathcal{E}_{a}^{l+2 m}(\Theta)} \leq k_{1}\left\|\left\{f_{j}\right\}\right\|_{\mathcal{E}_{a}^{l}(\Theta)}, \quad p \in S^{d-1},
$$

where $k_{1}>0$ does not depend on $u$ or $p$.

2. Making the Fourier transform with respect to $z$, we see that problem (7.31), (7.32) is equivalent to the following one:

$$
\begin{gathered}
A_{j}\left(D_{y}, \eta, p\right) \tilde{u}_{j}(y, \eta)=\tilde{f}_{j}(y, \eta), \quad y \in \theta_{j}, \quad \eta \in \mathbb{R}^{n-2}, \\
\left.\sum_{k, s}\left(B_{j \rho \mu k s}\left(D_{y}, \eta, p\right) \tilde{u}_{k}\right)\left(\mathcal{G}_{j \rho k s} y, \eta\right)\right|_{\gamma_{j \rho}}=0, \quad \eta \in \mathbb{R}^{n-2},
\end{gathered}
$$

where $\tilde{u}_{j}(y, \eta)$ is the Fourier transform of $u_{j}(y, z)$ with respect to $z$.

Set $\tilde{u}_{j}(y, \eta)=|(\eta, p)|^{-2 m} U_{j}(|(\eta, p)| y, \eta)$ and $\tilde{f}_{j}(y, \eta)=F_{j}(|(\eta, p)| y, \eta)$. Then problem (7.33), (7.34) takes the form

$$
\begin{gathered}
A_{j}\left(D_{Y}, \omega\right) U_{j}(Y, \eta)=F_{j}(Y, \eta), \quad y \in \theta_{j}, \quad \eta \in \mathbb{R}^{n-2}, \\
\left.\sum_{k, s}\left(B_{j \rho \mu k s}\left(D_{Y}, \omega\right) U_{k}\right)\left(\mathcal{G}_{j \rho k s} Y, \eta\right)\right|_{\gamma_{j \rho}}=0, \quad \eta \in \mathbb{R}^{n-2},
\end{gathered}
$$

where $\omega=(\eta, p) /|(\eta, p)| \in S^{n+d-3}$ and $Y=|(\eta, p)| y$.

It follows from the conditions of the lemma and from Theorem 2.1 that problem (7.35), (7.36) has a unique solution $U \in \mathcal{E}_{a}^{l+2 m}(\theta)$ for any right-hand side $\left\{F_{j}\right\} \in \mathcal{E}_{a}^{l}(\theta)$ and

$$
\|U\|_{\mathcal{E}_{a}^{l+2 m}(\theta)} \leq k_{2}\left\|\left\{F_{j}\right\}\right\|_{\mathcal{E}_{a}^{l}(\theta)}, \quad \omega \in S^{n+d-3},
$$

where $k_{2}>0$ does not depend on $u$ or $\omega$.

Thus, the lemma will be proved if we show that

$$
\begin{aligned}
\left\|\left\{f_{j}\right\}\right\|_{\mathcal{E}_{a}^{l}(\Theta)}^{2} & \approx \int_{\mathbb{R}^{n-2}}|(\eta, p)|^{-2(a-l+1)}\left\|\left\{F_{j}(\cdot, \eta)\right\}\right\|_{\mathcal{E}_{a}^{l}(\theta)}^{2} d \eta, \\
\|u\|_{\mathcal{E}_{a}^{l+2 m}(\Theta)}^{2} & \approx \int_{\mathbb{R}^{n-2}}|(\eta, p)|^{-2(a-l+1)}\|U(\cdot, \eta)\|_{\mathcal{E}_{a}^{l+2 m}(\theta)}^{2} d \eta ;
\end{aligned}
$$

here the symbol $\approx$ between two expressions means that the first expression can be estimated from below and from above by the second expression with positive constants independent of $p \in S^{d-1}$.

3. Let us prove relation (7.37). Using the Parseval equality, we have

$$
\begin{aligned}
\left\|f_{j}\right\|_{E_{a}^{l}\left(\Theta_{j}\right)}^{2} & =\sum_{|\alpha|+|\beta| \leq l} \int_{\theta_{j}} \int_{\mathbb{R}^{n-2}}|y|^{2 a}\left(|y|^{2(|\alpha|+|\beta|-l)}+1\right)\left|D_{y}^{\alpha} D_{z}^{\beta} f_{j}(y, z)\right|^{2} d y d z \\
& =\sum_{|\alpha|+|\beta| \leq l} \int_{\theta_{j}} \int_{\mathbb{R}^{n-2}}|y|^{2 a}\left(|y|^{2(|\alpha|+|\beta|-l)}+1\right)\left|\eta^{\beta}\right|^{2}\left|D_{y}^{\alpha} \tilde{f}_{j}(y, \eta)\right|^{2} d y d \eta .
\end{aligned}
$$


Using Fubini's theorem and making the change of variables $Y=|(\eta, p)| y$ for each fixed $\eta$, we obtain

$$
\begin{aligned}
\left\|f_{j}\right\|_{E_{a}^{l}\left(\Theta_{j}\right)}^{2}= & \sum_{|\alpha|+|\beta| \leq l} \int_{\mathbb{R}^{n-2}} \int_{\theta_{j}}|(\eta, p)|^{-2(a+1-|\alpha|)}|Y|^{2 a} \\
& \times\left(|(\eta, p)|^{-2(|\alpha|+|\beta|-l)}|Y|^{2(|\alpha|+|\beta|-l)}+1\right)\left|\eta^{\beta}\right|^{2}\left|D_{Y}^{\alpha} F(Y, \eta)\right|^{2} d Y d \eta \\
= & \sum_{|\alpha| \leq l} \sum_{\nu=0}^{l-|\alpha|} \int_{\mathbb{R}^{n-2}} \int_{\theta_{j}} \Phi_{\alpha \nu}(\eta, p, Y)\left|D_{Y}^{\alpha} F(Y, \eta)\right|^{2} d Y d \eta
\end{aligned}
$$

where

$$
\Phi_{\alpha \nu}(\eta, p, Y)=\sum_{|\beta|=\nu}|(\eta, p)|^{-2(a-l+1)}|Y|^{2 a} \frac{\left|\eta^{\beta}\right|^{2}}{|(\eta, p)|^{2|\beta|}}\left(|Y|^{2(|\alpha|+|\beta|-l)}+|(\eta, p)|^{2(|\alpha|+|\beta|-l)}\right) .
$$

Note that $p \in S^{d-1}$ and $|\alpha|+|\beta|-l \leq 0$, which implies that

$$
|(\eta, p)|^{2(|\alpha|+|\beta|-l)} \leq 1
$$

Therefore,

$$
|Y|^{2(|\alpha|+|\beta|-l)}+|(\eta, p)|^{2(|\alpha|+|\beta|-l)} \leq|Y|^{2(|\alpha|+|\beta|-l)}+1 .
$$

Furthermore, since $p \in S^{d-1}$, we have

$$
\sum_{|\beta|=\nu} \frac{\left|\eta^{\beta}\right|^{2}}{|(\eta, p)|^{2|\beta|}} \leq k_{3} \quad\left(\eta \in \mathbb{R}^{n-2}, p \in S^{d-1}, \nu=0, \ldots, l\right),
$$

where $k_{3}>0$ does not depend on $\eta$ or $p$.

Relations (7.39)-(7.41) imply that

$$
\begin{aligned}
& \quad\left\|f_{j}\right\|_{E_{a}^{l}\left(\Theta_{j}\right)}^{2} \\
& \leq k_{3} \sum_{|\alpha| \leq l} \sum_{\nu=0}^{l-|\alpha|} \int_{\mathbb{R}^{n-2}} \int_{\theta_{j}}|(\eta, p)|^{-2(a-l+1)}|Y|^{2 a}\left(|Y|^{2(|\alpha|+\nu-l)}+1\right)\left|D_{Y}^{\alpha} F_{j}(Y, \eta)\right|^{2} d Y d \eta .
\end{aligned}
$$

Clearly,

$$
|Y|^{2(|\alpha|-l)}+1 \leq \sum_{\nu=0}^{l-|\alpha|}|Y|^{2(|\alpha|+\nu-l)} \leq k_{4}\left(|Y|^{2(|\alpha|-l)}+1\right), \quad Y \in \mathbb{R}^{2},
$$

where $k_{4}>0$ does not depend on $Y$.

Inequalities (7.42) and (7.43) imply that

$$
\left\|f_{j}\right\|_{E_{a}^{l}\left(\Theta_{j}\right)}^{2} \leq k_{3} k_{4} \int_{\mathbb{R}^{n-2}}|(\eta, p)|^{-2(a-l+1)}\left\|F_{j}(\cdot, \eta)\right\|_{E_{a}^{l}\left(\theta_{j}\right)}^{2} d \eta .
$$

Now we estimate the norm $\left\|f_{j}\right\|_{E_{a}^{l}\left(\Theta_{j}\right)}^{2}$ from below. To do this, we write it as follows:

$$
\begin{aligned}
\left\|f_{j}\right\|_{E_{a}^{l}\left(\Theta_{j}\right)}^{2}=\sum_{|\alpha| \leq l} \sum_{\nu=0}^{l-|\alpha|}\left(\int_{|\eta|<1} \int_{\theta_{j}} \Phi_{\alpha \nu}(\eta, p, Y)\left|D_{Y}^{\alpha} F(Y, \eta)\right|^{2} d Y d \eta\right. \\
\left.\quad+\int_{|\eta|>1} \int_{\theta_{j}} \Phi_{\alpha \nu}(\eta, p, Y)\left|D_{Y}^{\alpha} F(Y, \eta)\right|^{2} d Y d \eta\right) .
\end{aligned}
$$


For $|\eta|<1$, we have

$$
\begin{aligned}
& \sum_{\nu=0}^{l-|\alpha|} \sum_{|\beta|=\nu} \frac{\left|\eta^{\beta}\right|^{2}}{|(\eta, p)|^{2|\beta|}}\left(|Y|^{2(|\alpha|+|\beta|-l)}+|(\eta, p)|^{2(|\alpha|+|\beta|-l)}\right) \\
& \quad \geq|Y|^{2(|\alpha|-l)}+|(\eta, p)|^{2(|\alpha|-l)} \geq k_{5}\left(|Y|^{2(|\alpha|-l)}+1\right),
\end{aligned}
$$

where $k_{5}>0$ does not depend on $Y, \eta$, or $p$. Therefore,

$$
\begin{aligned}
& \sum_{|\alpha| \leq l} \sum_{\nu=0}^{l-|\alpha|} \int_{|\eta|<1} \int_{\theta_{j}} \Phi_{\alpha \nu}(\eta, p, Y)\left|D_{Y}^{\alpha} F(Y, \eta)\right|^{2} d Y d \eta \\
& \quad \geq k_{5} \sum_{|\alpha| \leq l} \int_{|\eta|<1} \int_{\theta_{j}}|(\eta, p)|^{-2(a-l+1)}|Y|^{2 a}\left(|Y|^{2(|\alpha|-l)}+1\right)\left|D_{Y}^{\alpha} F(Y, \eta)\right|^{2} d Y d \eta .
\end{aligned}
$$

For $|\eta|>1$, we have

$$
\sum_{|\beta|=\nu} \frac{\left|\eta^{\beta}\right|^{2}}{|(\eta, p)|^{2|\beta|}} \geq k_{6} \quad\left(p \in S^{d-1}, \nu=0, \ldots, l\right)
$$

where $k_{6}>0$ does not depend on $\eta$ or $p$. It follows from (7.43) and (7.47) that

$$
\begin{aligned}
& \sum_{|\alpha| \leq l} \sum_{\nu=0}^{l-|\alpha|} \int_{|\eta|>1} \int_{\theta_{j}} \Phi_{\alpha \nu}(\eta, p, Y)\left|D_{Y}^{\alpha} F(Y, \eta)\right|^{2} d Y d \eta \\
& \quad \geq k_{6} \sum_{|\alpha| \leq l}^{l-|\alpha|} \sum_{\nu=0} \int_{|\eta|>1} \int_{\theta_{j}}|(\eta, p)|^{-2(a-l+1)}|Y|^{2(a+|\alpha|+\nu-l)}\left|D_{Y}^{\alpha} F(Y, \eta)\right|^{2} d Y d \eta \\
& \quad \geq k_{6} \sum_{|\alpha| \leq l} \int_{|\eta|>1} \int_{\theta_{j}}|(\eta, p)|^{-2(a-l+1)}|Y|^{2 a}\left(|Y|^{2(|\alpha|-l)}+1\right)\left|D_{Y}^{\alpha} F(Y, \eta)\right|^{2} d Y d \eta .
\end{aligned}
$$

Inequalities (7.45), (7.46), and (7.48) imply that

$$
\left\|f_{j}\right\|_{E_{a}^{l}\left(\Theta_{j}\right)}^{2} \geq k_{7} \int_{\mathbb{R}^{n-2}}|(\eta, p)|^{-2(a-l+1)}\left\|F_{j}(\cdot, \eta)\right\|_{E_{a}^{l}\left(\theta_{j}\right)}^{2} d \eta
$$

where $k_{7}>0$ does not depend on $p$.

Relation (7.37) follows from (7.44) and (7.49). Similarly, one can prove (7.38).

Now we can prove Theorem 7.1 .

Proof of Theorem 7.1. It is easy to see that $u(x)$ is a solution of the problem

$$
\begin{gathered}
A_{j}\left(D_{y}, D_{z}, p\right) u_{j}(y, z)=f_{j}(y, z), \quad(y, z) \in \Theta_{j}, \\
\left.B_{j \rho \mu}(p) u \equiv \sum_{k, s}\left(B_{j \rho \mu k s}\left(D_{y}, D_{z}, p\right) u_{k}\right)\left(\mathcal{G}_{j \rho k s} y, z\right)\right|_{\Gamma_{j \rho}}=f_{j \rho \mu}(y, z), \quad(y, z) \in \Gamma_{j \rho},
\end{gathered}
$$

iff the function $v(x)=u\left(|p|^{-1} x\right)$ is a solution of the problem

$$
\begin{aligned}
& A_{j}\left(D_{y}, D_{z}, p|p|^{-1}\right) v_{j}(y, z)=|p|^{-2 m} f_{j}\left(|p|^{-1} y,|p|^{-1} z\right), \quad(y, z) \in \Theta_{j} \text {, } \\
& \left.B_{j \rho \mu}\left(p|p|^{-1}\right) v \equiv \sum_{k, s}\left(B_{j \rho \mu k s}\left(D_{y}, D_{z}, p|p|^{-1}\right) v_{k}\right)\left(\mathcal{G}_{j \rho k s} y, z\right)\right|_{\Gamma_{j \rho}} \\
& =|p|^{-m_{j \rho \mu}} f_{j \rho \mu}\left(|p|^{-1} y,|p|^{-1} z\right), \quad(y, z) \in \Gamma_{j \rho},
\end{aligned}
$$

where $p \in \mathbb{R}^{d} \backslash\{0\}$. 
Further, we shall use the following inequalities:

$$
\begin{aligned}
& \left\|v_{j}\right\|_{E_{a}^{l+2 m}\left(\Theta_{j}\right)}^{2} \geq|p|^{2 a+n-2(l+2 m)}\left\|u_{j}\right\|_{V_{a}^{l+2 m}\left(\Theta_{j}\right)}^{2}, \quad p \in \mathbb{R}^{d} \backslash\{0\}, \\
& \left\|A_{j}\left(D_{y}, D_{z}, p|p|^{-1}\right) v_{j}\right\|_{E_{a}^{l}\left(\Theta_{j}\right)}^{2} \\
& \quad \leq k_{1}|p|^{2 a+n-2(l+2 m)}\left\|A_{j}\left(D_{y}, D_{z}, p\right) u_{j}\right\|_{V_{a}^{l}\left(\Theta_{j}\right)}^{2}, \quad p \in \mathbb{R}^{d} \backslash\{0\}, \\
& \quad\left\|B_{j \rho \mu}\left(p|p|^{-1}\right) v\right\|_{E_{a}^{l+2 m-m_{j \rho \mu}-1 / 2}\left(\Gamma_{j \rho}\right)}^{2} \\
& \quad \leq k_{2}|p|^{2 a+n-2(l+2 m)}\left\|B_{j \rho \mu}(p) u\right\|_{V_{a}^{l+2 m-m_{j \rho \mu}-1 / 2}\left(\Gamma_{j \rho}\right)}^{2}, \quad p \in \mathbb{R}^{d} \backslash\{0\},
\end{aligned}
$$

where $k_{1}, k_{2}>0$ do not depend on $u$ and $p$. To obtain inequality (7.50), we introduce the new variables $x^{\prime}=|p|^{-1} x$ and $y^{\prime}=|p|^{-1} y$, where $x=(y, z), x^{\prime}=\left(y^{\prime}, z^{\prime}\right), y, y^{\prime} \in \mathbb{R}^{2}$, and $z, z^{\prime} \in \mathbb{R}^{n-2}$. Then we have

$$
\begin{aligned}
& \left\|v_{j}\right\|_{E_{a}^{l+2 m}\left(\Theta_{j}\right)}^{2}=\sum_{|\alpha| \leq l+2 m} \int_{\Theta_{j}}|y|^{2 a}\left(|y|^{2(|\alpha|-l-2 m)}+1\right)\left|D_{x}^{\alpha} u_{j}\left(|p|^{-1} x\right)\right|^{2} d x \\
& \quad=|p|^{2 a+n-2(l+2 m)} \sum_{|\alpha| \leq l+2 m} \int_{\Theta_{j}}\left|y^{\prime}\right|^{2 a}\left(\left|y^{\prime}\right|^{2(|\alpha|-l-2 m)}+|p|^{2(l+2 m-|\alpha|)}\right)\left|D_{x^{\prime}}^{\alpha} u_{j}\left(x^{\prime}\right)\right|^{2} d x^{\prime} \\
& \quad \geq|p|^{2 a+n-2(l+2 m)}\left\|u_{j}\right\|_{V_{a}^{l+2 m}\left(\Theta_{j}\right)}^{2} .
\end{aligned}
$$

Using Lemma 7.1, similarly to (7.50), we derive (7.51).

To obtain inequality (7.52), one can use the equivalent norms in $E_{a}^{k-1 / 2}\left(\Gamma_{j \rho}\right)$ and $H_{a}^{k-1 / 2}\left(\Gamma_{j} \rho\right)$ given by

$$
\begin{aligned}
&\|u\|_{E_{a}^{k-1 / 2}\left(\Gamma_{j \rho}\right)}^{\prime}=\left(\left.\sum_{|\alpha|=k-1} \int_{\Gamma_{j \rho}} \int_{\Gamma_{j \rho}}|| y_{1}\right|^{a} D^{\alpha} u\left(x_{1}\right)-\left.\left|y_{2}\right|^{a} D^{\alpha} u\left(x_{2}\right)\right|^{2} \frac{d x_{1} d x_{2}}{\left|x_{1}-x_{2}\right|^{n}}\right. \\
&\left.+\sum_{|\alpha| \leq k-1} \int_{\Gamma_{j \rho}}|y|^{2 a}\left(|y|^{2(|\alpha|-k+1 / 2)}+1\right)\left|D^{\alpha} u(x)\right|^{2} d x\right)^{1 / 2}, \\
&\|u\|_{H_{a}^{k-1 / 2}\left(\Gamma_{j \rho}\right)}^{\prime}= \\
&\left.\quad \sum_{|\alpha|=k-1} \int_{\Gamma_{j \rho}} \int_{\Gamma_{j \rho}}|| y_{1}\right|^{a} D^{\alpha} u\left(x_{1}\right)-\left.\left|y_{2}\right|^{a} D^{\alpha} u\left(x_{2}\right)\right|^{2} \frac{d x_{1} d x_{2}}{\left|x_{1}-x_{2}\right|^{n}} \\
&\left.+\sum_{|\alpha| \leq k-1} \int_{\Gamma_{j \rho}}|y|^{2(a+|\alpha|-k+1 / 2)}\left|D^{\alpha} u(x)\right|^{2} d x\right)^{1 / 2}
\end{aligned}
$$

(see Lemmas 9.1 and 1.3 in 20]) and Lemma 7.3 .

Now the assertion of the theorem follows from Lemma 7.5 and inequalities (7.50), (7.51), and (7.52).

Further, we prove an analog of Corollary 2.1. Introduce the linear bounded operator $\mathcal{L}_{g}^{\prime \prime}(p): \mathcal{V}_{a}^{l+2 m}(\Theta) \rightarrow \mathcal{V}_{a}^{l}(\Theta, \Gamma)$ by the formula

$$
\mathcal{L}_{g}^{\prime \prime}(p) v=\mathcal{L}_{g}(p) v+\eta\left(\mathcal{L}_{g}^{\prime}(p)-\mathcal{L}_{g}(p)\right) v,
$$

where $\eta$ is the same function as in Sec. 2.3 ,

Corollary 7.1. Let the conditions of Theorem 7.1 hold. Then the operator $\mathcal{L}_{g}^{\prime \prime}(p)$ : $\mathcal{V}_{a}^{l+2 m}(\Theta) \rightarrow \mathcal{V}_{a}^{l}(\Theta, \Gamma)$ is an isomorphism for all sufficiently small $\delta>0$ and $p \in \mathbb{R}^{d} \backslash\{0\}$ 
and

$$
\|u\|_{\mathcal{V}_{a}^{l+2 m}(\Theta)} \leq c\left\|\mathcal{L}_{g}^{\prime \prime}(p) u\right\|_{\mathcal{V}_{a}^{l}(\Theta, \Gamma)}, \quad p \in \mathbb{R}^{d} \backslash\{0\}
$$

where $c>0$ does not depend on $u$ or $p$.

Proof. By Theorem 7.1, there is a bounded operator $\mathcal{L}_{g}^{-1}(p)$ and estimate (7.30) holds. We have

$$
\mathcal{L}_{g}^{\prime \prime}(p) \mathcal{L}_{g}^{-1}(p)=\mathcal{I}+\mathcal{M}(p)
$$

where $\mathcal{I}$ denotes the identity operator on $\mathcal{V}_{a}^{l}(\Theta, \Gamma)$ and

$$
\mathcal{M}(p)=\eta\left(\mathcal{L}_{g}^{\prime}(p)-\mathcal{L}_{g}(p)\right) \mathcal{L}_{g}^{-1}(p)
$$

It follows from (7.14) that

$$
\left\|\left.u\right|_{\mathcal{G}_{j \rho k s}\left(\Gamma_{i}\right)}\right\|_{V_{a}^{l+2 m-m_{j \rho \mu}-1 / 2}\left(\mathcal{G}_{j \rho k s}\left(\Gamma_{i}\right)\right)} \leq k_{1}\|u\|_{V_{a}^{l+2 m-m_{j \rho \mu}}\left(\Theta_{k}\right)},
$$

where $k_{1}, k_{2}, \ldots>0$ do not depend on $u$ or $p$. Therefore, similarly to the proof of Corollary 2.1, we obtain

$$
\|\mathcal{M}(p) f\|_{\mathcal{V}_{a}^{l}(\Theta, \Gamma)} \leq k_{2} \delta\left\|\mathcal{L}_{g}^{-1}(p) f\right\|_{\mathcal{V}_{a}^{l+2 m}(\Theta)} .
$$

Combining this inequality with (7.30) yields

$$
\|\mathcal{M}(p) f\|_{\mathcal{V}_{a}^{l}(\Theta, \Gamma)} \leq k_{3} \delta\|f\|_{\mathcal{V}_{a}^{l}(\Theta, \Gamma)} \cdot
$$

If $\delta>0$ is so small that $k_{3} \delta \leq 1 / 2$, then there exists the inverse operator $(\mathcal{I}+\mathcal{M}(p))^{-1}$ bounded in the norms $\|\cdot\|_{\mathcal{V}_{a}^{l}(\Theta, \Gamma)}$ uniformly with respect to $p \in \mathbb{R}^{d} \backslash\{0\}$.

Clearly, the operator $\mathcal{L}_{g}^{-1}(p)(\mathcal{I}+\mathcal{M}(p))^{-1}$ is the right inverse for $\mathcal{L}_{g}^{\prime \prime}(p)$ and

$$
\left\|\mathcal{L}_{g}^{-1}(p)(\mathcal{I}+\mathcal{M}(p))^{-1} f\right\|_{\mathcal{V}_{a}^{l+2 m}(\Theta)} \leq k_{4}\|f\|_{\mathcal{V}_{a}^{l}(\Theta, \Gamma)}, \quad p \in \mathbb{R}^{d} \backslash\{0\} .
$$

Similarly, one can prove the existence of a left inverse operator for $\mathcal{L}_{g}^{\prime \prime}(p)$.

7.4. Now we fix an arbitrary point $g \in K_{2}$. Similarly to Sec. 1.4, we arrive at the following model operator:

$$
\begin{aligned}
\mathcal{L}_{g}(p): V_{a}^{l+2 m}\left(\mathbb{R}_{+}^{n}\right) \rightarrow & \mathcal{V}_{a}^{l}\left(\mathbb{R}_{+}^{n}, \Gamma\right) \\
& =V_{a}^{l}\left(\mathbb{R}_{+}^{n}\right) \times V_{a}^{l+2 m-m_{i \mu}-1 / 2}\left(\mathbb{R}_{-}^{n-1}\right) \times V_{a}^{l+2 m-m_{i \mu}-1 / 2}\left(\mathbb{R}_{+}^{n-1}\right)
\end{aligned}
$$

given by

$$
\mathcal{L}_{g}(p) u=\left(A\left(D_{y}, D_{z}, p\right) u,\left.B_{i \mu 0}\left(D_{y}, D_{z}, p\right) u\right|_{\varphi=-\pi / 2},\left.B_{i \mu 0}\left(D_{y}, D_{z}, p\right) u\right|_{\varphi=\pi / 2}\right)
$$

(cf. (1.16) ). We assume that the space $V_{a}^{l+2 m}\left(\mathbb{R}_{+}^{n}\right)$ is equipped with the norm (7.3) and $\mathcal{V}_{a}^{l}\left(\mathbb{R}_{+}^{n}, \Gamma\right)$ with the norm

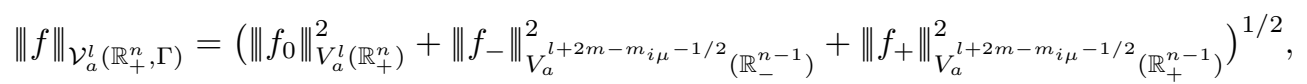

where $f=\left(f_{0}, f_{-}, f_{+}\right)$and the norm $\left\|f_{ \pm}\right\|_{V_{a}^{l+2 m-m_{i \mu}-1 / 2}}{\mathbb{\mathbb { R } _ { \pm } ^ { n - 1 }}}$ is defined by (7.4).

Analogously to Sec. 2.5, we consider the linear bounded operator

$$
\mathcal{L}_{g}(\omega): E_{a}^{l+2 m}\left(\mathbb{R}_{+}^{2}\right) \rightarrow \mathcal{E}_{a}^{l}\left(\mathbb{R}_{+}^{2}, \gamma\right)
$$

given by

$$
\mathcal{L}_{g}(\omega) V=\left(A\left(D_{y}, \omega\right) V,\left.B_{i \mu 0}\left(D_{y}, \omega\right) V\right|_{\mathbb{R}_{-}},\left.B_{i \mu 0}\left(D_{y}, \omega\right) V\right|_{\mathbb{R}_{+}}\right),
$$

where $\omega=(\eta, p) /|(\eta, p)| \in S^{n+d-3}$ (cf. (2.26) and (17.29) ).

Finally, we consider the analytic operator-valued function

$$
\hat{\mathcal{L}}_{g}(\lambda): W^{l+2 m}(-\pi / 2, \pi / 2) \rightarrow \mathcal{W}^{l}[-\pi / 2, \pi / 2]
$$

given by (2.27). 
The following theorem is an analog of Theorem 2.3 (cf. Theorem 9.1 and Corollary 9.1 in [20]).

Theorem 7.2. Let Conditions 7.1 and 7.2 hold. Assume that the line $\operatorname{Im} \lambda=a+1-l-2 m$ contains no eigenvalues of $\hat{\mathcal{L}}_{g}(\lambda)$ and $\operatorname{dim} \mathcal{N}\left(\mathcal{L}_{g}(\omega)\right)=\operatorname{codim} \mathcal{R}\left(\mathcal{L}_{g}(\omega)\right)=0$ for any $\omega \in S^{n+d-3}$. Then the operator $\mathcal{L}_{g}(p): \mathcal{V}_{a}^{l+2 m}\left(\mathbb{R}_{+}^{n}\right) \rightarrow \mathcal{V}_{a}^{l}\left(\mathbb{R}_{+}^{n}, \Gamma\right)$ is an isomorphism for $p \in \mathbb{R}^{d} \backslash\{0\}$ and

$$
\|u\|_{\mathcal{V}_{a}^{l+2 m}\left(\mathbb{R}_{+}^{n}\right)} \leq c\left\|\mathcal{L}_{g}(p) u\right\|_{\mathcal{V}_{a}^{l}\left(\mathbb{R}_{+}^{n}, \Gamma\right)}, \quad p \in \mathbb{R}^{d} \backslash\{0\},
$$

where $c>0$ does not depend on $u$ or $p$.

The proof is similar to the proof of Theorem 7.1 .

\section{Solvability of NONLOCAL ELliptiC PROBLEMS With A PARAMETER}

8.1. In this section, we prove the unique solvability of nonlocal elliptic problems with a parameter in bounded domains.

Lemma 8.1. Let $H$ be a Hilbert space and I the identity operator on $H$. Let $M_{\varepsilon}(p)$ and $S_{\varepsilon}(p)\left(\varepsilon>0, p \in \mathbb{R}^{d}\right.$, and $|p|$ is sufficiently large) be families of bounded operators on $H$ such that

$$
\left\|M_{\varepsilon}(p)\right\| \leq c_{1} \varepsilon, \quad\left\|S_{\varepsilon}(p)\right\| \leq c_{2}, \quad\left\|S_{\varepsilon}^{2}(p)\right\| \leq c_{3}|p|^{-1},
$$

where $c_{1}, c_{2}, c_{3}>0$ do not depend on $\varepsilon$ or $p$. Then the operators

$$
L_{\varepsilon}(p)=I+M_{\varepsilon}(p)+S_{\varepsilon}(p)
$$

have bounded inverse operators $L_{\varepsilon}^{-1}(p)$ and the estimate

$$
\left\|L_{\varepsilon}^{-1}(p)\right\| \leq c_{4}
$$

holds for sufficiently small $\varepsilon>0$ and sufficiently large $|p|$, where $c_{4}>0$ does not depend on $\varepsilon$ or $p$.

Proof. To prove the lemma, we will construct a right and a left inverse operator for $L_{\varepsilon}(p)$. We have

$$
L_{\varepsilon}(p)\left(I-\left(M_{\varepsilon}(p)+S_{\varepsilon}(p)\right)\right)=I-M_{\varepsilon}^{2}(p)-M_{\varepsilon}(p) S_{\varepsilon}(p)-S_{\varepsilon}(p) M_{\varepsilon}(p)-S_{\varepsilon}^{2}(p) .
$$

It follows from (8.1) that

for sufficiently large $|p|$ and

$$
\left\|S_{\varepsilon}^{2}(p)\right\| \leq 1 / 6
$$

$$
\left\|M_{\varepsilon}(p)\right\| \leq \min \left(1 / \sqrt{6}, 1 /\left(12 c_{2}\right)\right)
$$

for sufficiently small $\varepsilon$. Therefore,

$$
\left\|M_{\varepsilon}^{2}(p)+M_{\varepsilon}(p) S_{\varepsilon}(p)+S_{\varepsilon}(p) M_{\varepsilon}(p)+S_{\varepsilon}^{2}(p)\right\| \leq 1 / 2 .
$$

Thus, the operators $\left(I-M_{\varepsilon}^{2}(p)-M_{\varepsilon}(p) S_{\varepsilon}(p)-S_{\varepsilon}(p) M_{\varepsilon}(p)-S_{\varepsilon}^{2}(p)\right)^{-1}$ exist and are uniformly bounded with respect to $\varepsilon$ and $p$. Combining this fact with the uniform boundedness of the operators $I-\left(M_{\varepsilon}(p)+S_{\varepsilon}(p)\right)$, we see that each of the operators

$$
L_{\varepsilon}^{-1}(p)=\left(I-\left(M_{\varepsilon}(p)+S_{\varepsilon}(p)\right)\right)\left(I-M_{\varepsilon}^{2}(p)-M_{\varepsilon}(p) S_{\varepsilon}(p)-S_{\varepsilon}(p) M_{\varepsilon}(p)-S_{\varepsilon}^{2}(p)\right)^{-1}
$$

is the right inverse for the operator $L_{\varepsilon}(p)$ and

$$
\left\|L_{\varepsilon}^{-1}(p)\right\| \leq c_{4} .
$$

Similarly, one can prove that there exist uniformly bounded left inverse operators for the operators $L_{\varepsilon}(p)$. 
Lemma 8.2. Let Conditions 7.1, 7.2, 1.3, and 1.4 hold. Assume that the line $\operatorname{Im} \lambda=$ $a+1-l-2 m$ contains no eigenvalues of $\hat{\mathcal{L}}_{g}(\lambda)$ for any $g \in K$ and $\operatorname{dim} \mathcal{N}\left(\mathcal{L}_{g}(\omega)\right)=$ $\operatorname{codim} \mathcal{R}\left(\mathcal{L}_{g}(\omega)\right)=0$ for any $g \in K$ and $\omega \in S^{n+d-3}$. Then there is a number $p_{0}>0$ such that the operator $\mathbf{L}^{1}(p),|p| \geq p_{0}$, has a bounded inverse and

$$
c_{1}\left\|\mathbf{L}^{1}(p) u\right\|_{\mathcal{H}_{a}^{l}(Q, \Gamma)} \leq\|u\|_{H_{a}^{l+2 m}(Q)} \leq c_{2}\left\|\mathbf{L}^{1}(p) u\right\|_{\mathcal{H}_{a}^{l}(Q, \Gamma)}, \quad|p| \geq p_{0},
$$

where $c_{1}, c_{2}>0$ do not depend on $u$ or $p$.

Proof. 1. The first inequality in (8.2) follows from the definition of the norms $\|\cdot\|$, from Lemma 7.1, and from estimate (7.15). To prove the second inequality in (8.2), we repeat the proof of Lemma 4.3, replacing there the norms $\|\cdot\|$ by the norms $\|\cdot\|$, Corollary 2.1 and Theorem 2.3 by Corollary 7.1 and Theorem 7.2 respectively, and the results on elliptic problems in the interior of the domain and near a smooth part of the boundary by the corresponding results on elliptic problems with a parameter [2] and taking into account estimate (7.15). Then we obtain the following a priori estimate:

$$
\|u\|_{H_{a}^{l+2 m}(Q)} \leq k_{1}\left(\left\|\mathbf{L}^{1}(p) u\right\|_{\mathcal{H}_{a}^{l}(Q, \Gamma)}+\|u\|_{H_{a}^{l+2 m-1}(Q)}\right), \quad p \in \mathbb{R}^{d} \backslash\{0\},
$$

where $k_{1}>0$ does not depend on $u$ and $p$. Combining this estimate with the relation

$$
\|u\|_{H_{a}^{l+2 m-1}(Q)} \leq|p|^{-1}\|u\|_{H_{a}^{l+2 m}(Q)}
$$

and taking $|p| \geq p^{\prime}$, where $p^{\prime}>0$ is sufficiently large, we obtain the second inequality in (8.2).

2. It remains to prove the existence of a right inverse operator for $\mathbf{L}^{1}(p)$.

Using the notation from the proof of Lemma 5.2, we introduce the operator

$$
R_{\mathcal{K}_{1}}(p) f=\sum_{t}\left(U^{t}\right)^{-1}\left(\hat{\xi}^{t}\left(\mathcal{L}_{g^{t}}^{\prime \prime}(p)\right)^{-1} F^{t}\left(\sum_{q} \xi_{q}^{t} f\right)\right)
$$

(cf. (5.9)). Similarly to (5.24), we prove that

$$
\mathbf{L}^{1}(p) R_{\mathcal{K}_{1}}(p) f=\xi_{0} f+T_{\mathcal{K}_{1}}(p) f,
$$

where $T_{\mathcal{K}_{1}}(p): \mathcal{H}_{a}^{l}(Q, \Gamma) \rightarrow \mathcal{H}_{a}^{l}(Q, \Gamma)$ is a bounded operator such that

$$
\begin{gathered}
\left\|T_{\mathcal{K}_{1}}(p) f\right\|_{\mathcal{H}_{a}^{l}(Q, \Gamma)} \leq c_{1}\|f\|_{\mathcal{H}_{a}^{l}(Q, \Gamma)}, \\
\left\|T_{\mathcal{K}_{1}}^{2}(p) f\right\|_{\mathcal{H}_{a}^{l}(Q, \Gamma)} \leq c_{2}|p|^{-1}\|f\|_{\mathcal{H}_{a}^{l}(Q, \Gamma)}
\end{gathered}
$$

and $c_{1}, c_{2}, \ldots>0$ do not depend on $f, p$, and on the number $\varepsilon$ in the definition of the function $\xi,|p| \geq p^{\prime}$.

Estimate (8.4) follows from Corollary 7.1 and inequalities (7.6) and (7.15). Let us prove (8.5). By analogy with the operators $\mathcal{T}_{j \rho \mu}^{t}, A_{k}^{\prime \prime}$, and $T_{i}, i=1,2,3$, from the proof of Lemma [5.2] we consider the corresponding operators $\mathcal{T}_{j \rho \mu}^{t}(p), A_{k}^{\prime \prime}(p)$ and $T_{i}(p), i=$ $1,2,3$, depending on the parameter $p$. First, we estimate the norm of $T_{3}^{2}(p) f$. Introduce functions $\psi_{k}^{t} \in C_{0}^{\infty}\left(\Theta_{k}\right)$ such that $\psi_{k}^{t}\left(x^{\prime}\right)=1$ for $x^{\prime} \in \Omega_{k}^{t}$. Using inequality (5.20) with the norms $\|\cdot\|$ replaced by the norms $\|\cdot\|$, the equivalence of the norms $\|\cdot\|$ in the subspaces of $H_{a}^{l}\left(\Theta_{k}\right)$ and $W^{l}\left(\Theta_{k}\right)$ consisting of compactly supported functions vanishing near the edge $\mathcal{P}$, Theorem 4.1 in [2], equality (5.22), Leibniz' formula, inequality (7.6), 
and Corollary 7.1, we have

$$
\begin{aligned}
\left\|\mathcal{T}_{j \rho \mu}^{t}(p)\left(\mathcal{L}_{g^{t}}^{\prime \prime}(p)\right)^{-1} F^{t}\left(\sum_{q} \xi_{q}^{t} T_{3}(p) f\right)\right\| \|_{V_{a}^{l+2 m-m_{j \rho \mu}-1 / 2}\left(\Gamma_{j \rho}\right)} \\
\leq k_{1} \sum_{k}\|\| A_{k}^{\prime \prime}(p)\left(\psi_{k}^{t}\left[\left(\mathcal{L}_{g^{t}}^{\prime \prime}(p)\right)^{-1} F^{t}\left(\sum_{q} \xi_{q}^{t} T_{3}(p) f\right)\right]_{k}\right)\|\|_{V_{a}^{l}\left(\Theta_{k}\right)} \\
\leq k_{2}\left\|\left(\mathcal{L}_{g^{t}}^{\prime \prime}(p)\right)^{-1} F^{t}\left(\sum_{q} \xi_{q}^{t} T_{3}(p) f\right)\right\|\left\|_{\mathcal{V}_{a}^{l+2 m-1}(\Theta)} \leq k_{3}|p|^{-1}\right\| f \|_{\mathcal{H}_{a}^{l}(Q, \Gamma)},
\end{aligned}
$$

where $p \in \mathbb{R}^{d} \backslash\{0\}$ and $k_{1}, \ldots, k_{4}>0$ do not depend on $f, p$, or $\varepsilon$.

The latter inequality implies that

$$
\left\|T_{3}^{2}(p) f\right\|_{\mathcal{H}_{a}^{l}(Q, \Gamma)} \leq k_{4}|p|^{-1}\|f\|_{\mathcal{H}_{a}^{l}(Q, \Gamma)}, \quad p \in \mathbb{R}^{d} \backslash\{0\} .
$$

Similarly, we estimate the norm of $T_{1}^{2}(p) f$. The analogous estimate for $T_{2}(p) f$ is evident. Thus, we obtain inequality (8.5) for $T_{\mathcal{K}_{1}}(p)=T_{1}(p)+T_{2}(p)+T_{3}(p)$.

Let $\zeta$ be a function defined in (5.28). Set $\zeta_{1}=1-\zeta$. Since $\zeta(x)=1$ for $x \in \mathcal{K}_{1}^{2 \varepsilon}$, it follows that $\operatorname{supp} \zeta_{1} \subset \bar{Q} \backslash \mathcal{K}_{1}^{2 \varepsilon}$. Introduce a function $\hat{\zeta}_{1} \in C^{\infty}\left(\mathbb{R}^{n}\right)$ such that $\hat{\zeta}_{1}(x)=1$ for $x \in \bar{Q} \backslash \mathcal{K}_{1}^{2 \varepsilon}$ and supp $\hat{\zeta}_{1} \subset \bar{Q} \backslash \mathcal{K}_{1}^{\varepsilon}$.

Due to Theorem 10.4 in [20], there exists a bounded operator $\mathbf{R}^{0}(p)$ such that

$$
\mathbf{L}_{0}(p) \mathbf{R}_{0}(p) f=f
$$

for $f \in \mathcal{H}_{a}^{l}(Q, \Gamma)$, supp $f \subset \bar{Q} \backslash \mathcal{K}_{1}^{2 \varepsilon}$, provided that $|p| \geq p^{\prime \prime}$, where $p^{\prime \prime} \geq p^{\prime}$ is sufficiently large. Thus, we can set

$$
R(p) f=R_{\mathcal{K}_{1}}(p) f+R_{\mathcal{K}_{1}}(p)(\eta f)+\hat{\zeta}_{1} \mathbf{R}^{0}(p)\left(\zeta_{1} f\right),
$$

where $\eta(x)=\zeta_{0}(x)\left(1-\xi_{0}(x)\right) / \xi_{0}(x)$ for $x \in \mathcal{K}_{1}^{4 \varepsilon}$ and $\eta(x)=0$ for $x \notin \mathcal{K}_{1}^{4 \varepsilon}$ (cf. (55.30) ). Since $\operatorname{supp} \hat{\zeta}_{1} \subset \bar{Q} \backslash \mathcal{K}_{1}^{\varepsilon}$, we have

$$
B_{i \mu}^{1}(p)\left(\hat{\zeta}_{1} \mathbf{R}^{0}(p)\left(\zeta_{1} f\right)\right)=0
$$

and hence

$$
\mathbf{L}^{1}(p) R(p) f=\mathbf{L}^{1}(p) R_{\mathcal{K}_{1}}(p) f+\mathbf{L}^{1}(p) R_{\mathcal{K}_{1}}(p)(\eta f)+\mathbf{L}^{0}(p)\left(\hat{\zeta}_{1} \mathbf{R}^{0}(p)\left(\zeta_{1} f\right)\right) .
$$

Combining this relation with (8.3) and using Leibniz' formula and Lemmas 7.1 and 7.2 , we obtain

$$
\begin{aligned}
\mathbf{L}^{1}(p) R(p) f & =\xi_{0} f+T_{\mathcal{K}_{1}}(p) f+\zeta_{0}\left(1-\xi_{0}\right) f+T_{\mathcal{K}_{1}}(p)(\eta f)+\zeta_{1} f+T(p) f \\
& =f+T_{\mathcal{K}_{1}}(p) f+M(p) f+T(p) f
\end{aligned}
$$

or, equivalently,

$$
\mathbf{L}^{1}(p) R(p)=\mathbf{I}+T_{\mathcal{K}_{1}}(p)+M(p)+T(p),
$$

where

$$
M(p) f=T_{\mathcal{K}_{1}}(p)(\eta f),
$$

while $T(p): \mathcal{H}_{a}^{l}(Q, \Gamma) \rightarrow \mathcal{H}_{a}^{l}(Q, \Gamma)$ is a bounded operator such that

$$
\|T(p) f\|_{\mathcal{H}_{a}^{l}(Q, \Gamma)} \leq k_{1}|p|^{-1}\|f\|_{\mathcal{H}_{a}^{l}(Q, \Gamma)},
$$

where $k_{1}=k_{1}(\varepsilon)>0$ does not depend on $f$ or $p$.

By inequality (8.4), we have

$$
\|M(p) f\|_{\mathcal{H}_{a}^{l}(Q, \Gamma)} \leq c_{3}\|\eta f\|_{\mathcal{H}_{a}^{l}(Q, \Gamma)} .
$$


However, $\left(1-\xi_{0}(x)\right) / \xi_{0}(x)=0$ for $x \in \mathcal{K}_{1}$, while the function $\zeta_{0}$ is supported in $\mathcal{K}_{1}^{4 \varepsilon}$ and satisfies the inequality in (5.27). Therefore, it follows from the last estimate, from Lemmas 4.1 and 4.2 and from Remark 4.1 that

$$
\|M(p) f\|_{\mathcal{H}_{a}^{l}(Q, \Gamma)} \leq c_{4} \varepsilon\|f\|_{\mathcal{H}_{a}^{l}(Q, \Gamma)} .
$$

By virtue of inequalities (8.4), (8.5), and (8.9) and Lemma 8.1, the operator

$$
\left(\mathbf{I}+T_{\mathcal{K}_{1}}(p)+M(p)\right)^{-1}
$$

exists and is bounded in the norms $\|\cdot\|_{\mathcal{H}_{a}^{l}(Q, \Gamma)}$, uniformly with respect to $p,|p| \geq p^{\prime \prime \prime}$, where $p^{\prime \prime \prime} \geq p^{\prime \prime}$ is sufficiently large, provided that $\varepsilon>0$ is a sufficiently small fixed number. Therefore, relation (8.7) is equivalent to the following one:

$$
\mathbf{L}^{1}(p) R(p)\left(\mathbf{I}+T_{\mathcal{K}_{1}}(p)+M(p)\right)^{-1}=\mathbf{I}+T^{\prime}(p),
$$

where

$$
T^{\prime}(p)=T(p)\left(\mathbf{I}+T_{\mathcal{K}_{1}}(p)+M(p)\right)^{-1} .
$$

By virtue of the uniform boundedness of the operator (8.10) and estimate (8.8), there is a sufficiently large number $p_{0} \geq p^{\prime \prime \prime}$ such that $\left\|T^{\prime}(p)\right\| \leq 1 / 2$ for $|p| \geq p_{0}$ (recall that $\varepsilon$ is fixed) and hence

$$
\mathbf{L}^{1}(p) R(p)\left(\mathbf{I}+T_{\mathcal{K}_{1}}(p)+M(p)\right)^{-1}\left(\mathbf{I}+T^{\prime}(p)\right)^{-1}=\mathbf{I} .
$$

Thus, we have proved the existence of the right inverse operator for $\mathbf{L}^{1}(p),|p| \geq p_{0}$. Combining this with the second estimate in (8.2), we complete the proof.

8.2. In this subsection, we generalize the result of the previous subsection to the operator $\mathbf{L}(p)$.

Theorem 8.1. Let Conditions 7.1 7.4, 1.3, and 1.4 hold. Assume that the line $\operatorname{Im} \lambda=$ $a+1-l-2 m$ contains no eigenvalues of $\hat{\mathcal{L}}_{g}(\lambda)$ for any $g \in K$ and $\operatorname{dim} \mathcal{N}\left(\mathcal{L}_{g}(\omega)\right)=$ $\operatorname{codim} \mathcal{R}\left(\mathcal{L}_{g}(\omega)\right)=0$ for any $g \in K$ and $\omega \in S^{n+d-3}$. Then the following assertions are true:

(1) there is a number $p_{1}>0$ such that the operator $\mathbf{L}(p),|p| \geq p_{1}$, has a bounded inverse and

$$
c_{1}\|\mathbf{L}(p) u\|_{\mathcal{H}_{a}^{l}(Q, \Gamma)} \leq\|u\|_{H_{a}^{l+2 m}(Q)} \leq c_{2}\|\mathbf{L}(p) u\|_{\mathcal{H}_{a}^{l}(Q, \Gamma)}, \quad|p| \geq p_{1},
$$

where $c_{1}, c_{2}>0$ do not depend on $u$ or $p$;

(2) the operator $\mathbf{L}(p)$ has the Fredholm property and ind $\mathbf{L}(p)=0$ for $p \in \mathbb{R}^{d}$.

To prove Theorem 8.1, we preliminarily consider the operators

$$
L_{t}(p)=\mathbf{L}^{1}(p)+t\left(\mathbf{L}(p)-\mathbf{L}^{1}(p)\right), \quad 0 \leq t \leq 1 .
$$

Clearly, $L_{0}(p)=\mathbf{L}^{1}(p), L_{1}(p)=\mathbf{L}(p)$.

Lemma 8.3. Let the conditions of Theorem 8.1 hold. Then there is a number $p_{1}>0$ such that the following estimates hold for $u \in H_{a}^{l+2 m}(Q)$ :

$$
c_{1}\left\|L_{t}(p) u\right\|_{\mathcal{H}_{a}^{l}(Q, \Gamma)} \leq\|u\|_{H_{a}^{l+2 m}(Q)} \leq c_{2}\left\|L_{t}(p) u\right\|_{\mathcal{H}_{a}^{l}(Q, \Gamma)}, \quad|p| \geq p_{1}, \quad 0 \leq t \leq 1,
$$

where $c_{1}, c_{2}>0$ do not depend on $u$, $p$, or $t$.

Proof. The definition of the norms $\|\cdot\|$, , Lemma 7.1, inequality (7.15), and Conditions 7.3 and 7.4 imply the first estimate in (8.12). 
Let us prove the second estimate in (8.12). Applying Lemma 8.2 and using Condition 7.3 and the fact that $0 \leq t \leq 1$, we have

$$
\begin{aligned}
& \|u\|_{H_{a}^{l+2 m}(Q)} \leq k_{1}\left\|L_{0}(p) u\right\|_{\mathcal{H}_{a}^{l}(Q, \Gamma)} \\
& \quad \leq k_{2}\left(\left\|L_{t}(p) u\right\|_{\mathcal{H}_{a}^{l}(Q, \Gamma)}+\sum_{i, \mu}\left\|B_{i \mu}^{2}(p)\right\|_{H_{a}^{l+2 m-m_{i \mu}-1 / 2}\left(\Gamma_{i}\right)}+\|u\|_{H_{a}^{l+2 m-1}(Q)}\right),
\end{aligned}
$$

where $|p| \geq p_{0}$ and $k_{1}, k_{2}, \ldots>0$ do not depend on $u, p$, or $t$.

Further, we can repeat the proof of inequalities (4.35) and (4.36), using Lemma 7.1 and estimate (7.15) and replacing the operators $B_{i \mu}^{2}$ by $B_{i \mu}^{2}(p)$, the norms $\|\cdot\|$ by the norms $\|\cdot\|$, Lemma 4.6 by Condition 7.4 , and the results on elliptic problems in the interior of the domain and near a smooth part of the boundary by the corresponding results for elliptic problems with a parameter [2]. Thus, we obtain

$$
\left\|B_{i \mu}^{2}(p) u\right\|_{H_{a}^{l+2 m-m_{i \mu}-1 / 2}\left(\Gamma_{i}\right)} \leq k_{3}\left(\left\|L_{t} u\right\|_{\mathcal{H}_{a}^{l}(Q, \Gamma)}+\|u\|_{H_{a}^{l+2 m-1}(Q)}\right) .
$$

Combining estimates (8.13) and (8.14) with Lemma 7.1 and taking $|p| \geq p_{1}$, where $p_{1} \geq p_{0}$ is sufficiently large, we complete the proof.

Now we will prove Theorem 8.1 using Lemmas 8.2 and 8.3 , and the method of continuation along the parameter $t$.

Proof of Theorem 8.1, 1. Applying Lemmas 8.2 and 8.3 , we see that the operator

$$
L_{t}(p)=L_{0}(p)\left(\mathbf{I}+t L_{0}^{-1}(p)\left(L_{1}(p)-L_{0}(p)\right)\right)
$$

has a bounded inverse for $0 \leq t \leq t_{1}=c_{1} /\left(4 c_{2}\right)$ with the norm $\left\|L_{t}^{-1}(p)\right\| \leq c_{2}$. Therefore, the operator

$$
L_{t}(p)=L_{t_{1}}(p)\left(\mathbf{I}+\left(t-t_{1}\right) L_{t_{1}}^{-1}(p)\left(L_{1}(p)-L_{0}(p)\right)\right)
$$

has a bounded inverse for $t_{1} \leq t \leq 2 t_{1}$ with the norm $\left\|L_{t}^{-1}(p)\right\| \leq c_{2}$. Repeating this procedure finitely many times, we see that the operator $L_{1}(p)=\mathbf{L}(p)$ has a bounded inverse. Estimate (8.11) follows from (8.12) for $t=1$.

2. Fix $\hat{p} \subset \mathbb{R}^{d}$ such that $|\hat{p}| \geq p_{0}$. In this case, there exists a bounded operator $\left(\mathbf{L}^{1}(\hat{p})\right)^{-1}: \mathcal{H}_{a}^{l}(Q, \Gamma) \rightarrow H_{a}^{l+2 m}(Q)$ due to Lemma 8.2 Thus, we have

$$
\mathbf{L}^{1}(p)=\mathbf{L}^{1}(\hat{p})(\mathbf{I}+\mathbf{T}(p)),
$$

where

$$
\mathbf{T}(p)=\left(\mathbf{L}^{1}(\hat{p})\right)^{-1}\left(\mathbf{L}^{1}(p)-\mathbf{L}^{1}(\hat{p})\right) .
$$

Clearly, the operator $\mathbf{L}^{1}(p)-\mathbf{L}^{1}(\hat{p}): H_{a}^{l+2 m-1}(Q) \rightarrow \mathcal{H}_{a}^{l}(Q, \Gamma)$ is bounded. It follows from the compactness of the embedding $H_{a}^{l+2 m}(Q) \subset H_{a}^{l+2 m-1}(Q)$ that the operator $\mathbf{L}^{1}(p)-\mathbf{L}^{1}(\hat{p}): H_{a}^{l+2 m}(Q) \rightarrow \mathcal{H}_{a}^{l}(Q, \Gamma)$ is compact. Therefore, the operator $\mathbf{T}(p):$ $H_{a}^{l+2 m}(Q) \rightarrow H_{a}^{l+2 m}(Q)$ is also compact. By Theorem 13.2 in [18, the operator $\mathbf{I}+\mathbf{T}(p)$ has the Fredholm property and ind $(\mathbf{I}+\mathbf{T}(p))=0$. Now, applying Theorem 12.2 in [18, we see that the operator $\mathbf{L}^{1}(p)$ has the Fredholm property and

$$
\operatorname{ind} \mathbf{L}^{1}(p)=\operatorname{ind} \mathbf{L}^{1}(\hat{p})+\operatorname{ind}(\mathbf{I}+\mathbf{T}(p))=0 \text {. }
$$

Finally, we note that the fulfillment of Conditions 7.3 and 7.4 implies the fulfillment of Conditions 6.1 and 6.2. respectively. Therefore, by Theorem 6.3. the operator $\mathbf{L}(p)$ has the Fredholm property and

$$
\operatorname{ind} \mathbf{L}(p)=\operatorname{ind} \mathbf{L}^{1}(p)=0 .
$$


8.3. In this subsection, we consider an example of an elliptic problem with a parameter, having distributed nonlocal terms and satisfying Conditions 7.1] 7.4, 1.3, and 1.4.

Example 8.1. 1. In the notation of Example 6.1, we consider the following nonlocal problem:

$$
\begin{gathered}
-\Delta u+e^{i h} p^{2} u=f_{0}(x), \quad x \in Q, \\
\left.u\right|_{\Gamma_{l}}+B_{l}^{1} u+B_{l}^{2} u=f_{l}(x), \quad x \in \Gamma_{l}, l=1,2,
\end{gathered}
$$

where $-\pi / 2<h<\pi / 2$ and $p \geq 0$.

For each point $g \in \mathcal{K}_{1}$, the operator $\mathcal{L}_{g}(p)=\mathcal{L}(p): H_{a}^{2}(\Theta) \rightarrow \mathcal{H}_{a}^{0}(\Theta, \Gamma)$ given by (7.28) takes the form (cf. 6.14)

$$
\begin{array}{r}
\mathcal{L}(p) v=\left(-\Delta v+e^{i h} p^{2},\left.v(\varphi, r, z)\right|_{\Gamma_{11}}-\left.\alpha_{1} v(\varphi+\pi / 4, r, z)\right|_{\Gamma_{11}},\right. \\
\left.\left.v(\varphi, r, z)\right|_{\Gamma_{12}}-\left.\alpha_{2} v(\varphi-\pi / 4, r, z)\right|_{\Gamma_{12}}\right) .
\end{array}
$$

Hence, the operators

$$
\begin{gathered}
\mathcal{L}_{g}(\omega)=\mathcal{L}(\omega): E_{a}^{2}(\theta) \rightarrow \mathcal{E}_{a}^{0}(\theta, \gamma), \\
\hat{\mathcal{L}}_{g}(\lambda)=\hat{\mathcal{L}}(\lambda): W^{2}(-\pi / 4, \pi / 4) \rightarrow \mathcal{W}^{0}[-\pi / 4, \pi / 4]
\end{gathered}
$$

given by (7.29) and (2.2) have the form

$$
\begin{aligned}
\mathcal{L}(\omega) V=\left(-\Delta_{y} V+\left(\omega_{1}^{2}+e^{i h} \omega_{2}^{2}\right) V\right. & \\
& \left.\left.V(\varphi, r)\right|_{\gamma_{11}}-\left.\alpha_{1} V(\varphi+\pi / 4, r)\right|_{\gamma_{11}},\left.V(\varphi, r)\right|_{\gamma_{12}}-\left.\alpha_{2} V(\varphi-\pi / 4, r)\right|_{\gamma_{12}}\right)
\end{aligned}
$$

and

$$
\hat{\mathcal{L}}(\lambda) w=\left(-w_{\varphi \varphi}+\lambda^{2} w, w(-\pi / 4)-\alpha_{1} w(0), w(\pi / 4)-\alpha_{2} w(0)\right),
$$

respectively, where $\omega=\left(\omega_{1}, \omega_{2}\right) \in S^{1}$.

Let the numbers $a, \alpha_{1}, \alpha_{2}$ satisfy the following relations:

$$
0 \leq a \leq 2, \quad 0<\left|\alpha_{1}+\alpha_{2}\right|<2, \quad \pi / 4<\arctan \sqrt{4\left(\alpha_{1}+\alpha_{2}\right)^{-2}-1} .
$$

2. We claim that there is a number $h_{1}=h_{1}\left(\alpha_{1}, \alpha_{2}\right)>0$ such that the operator $\mathcal{L}(\omega)$, $\omega \in S^{1}$, is an isomorphism for $|h| \leq h_{1}$. To prove this fact, one must repeat the reasoning of Example 2.1. where the sesquilinear form (2.14) is replaced by the form

$$
b_{\mathcal{R}}[u, v]=\int_{\theta}\left(\sum_{i=1,2}\left(\mathcal{R}_{\theta} u\right)_{y_{i}} \overline{v_{y_{i}}}+\left(\omega_{1}^{2}+e^{i h} \omega_{2}^{2}\right) \mathcal{R}_{\theta} u \bar{v}\right) d y
$$

with the same domain $\mathrm{D}\left(b_{\mathcal{R}}\right)=\stackrel{\circ}{W}^{1}(\theta)$. Let us show that this sesquilinear form is again a closed sectorial form.

It follows from the Schwarz inequality and from (2.15) that

$$
\left|b_{\mathcal{R}}[u, v]\right| \leq k_{1}\|u\|_{{\stackrel{\circ}{W^{1}}(\theta)}}\|v\|_{{\stackrel{\circ}{W^{1}}(\theta)}},
$$

where $k_{1}>0$ does not depend on $u$ or $v$.

Introduce the isomorphism $\mathcal{U}: L_{2}(\theta) \rightarrow L_{2}\left(\theta_{1}\right) \times L_{2}\left(\theta_{1}\right)$ by the formula

$$
(\mathcal{U} u)_{i}(y)=u(\varphi+(i-1) d / 2, r), \quad y \in \theta_{1}, i=1,2,
$$

and let $R_{1}=\left(\begin{array}{cc}1 & \alpha_{1} \\ \alpha_{2} & 1\end{array}\right)$. Then, using (2.15) and (2.17), we obtain

$$
\begin{aligned}
\operatorname{Re} b_{\mathcal{R}}[u, u]=\int_{\theta_{1}}\left\{\sum _ { i } \left(\frac{\left(R_{1}+R_{1}^{*}\right)}{2}\right.\right. & \left.\left(\mathcal{U} u_{y_{i}}\right), \mathcal{U} u_{y_{i}}\right)_{\mathbb{C}^{2}}+\omega_{1}^{2}\left(\frac{\left(R_{1}+R_{1}^{*}\right)}{2} \mathcal{U} u, \mathcal{U} u\right)_{\mathbb{C}^{2}} \\
& \left.+\omega_{2}^{2}\left(\frac{\left.e^{i h} R_{1}+\left(e^{i h} R_{1}\right)^{*}\right)}{2} \mathcal{U} u, \mathcal{U} u\right)_{\mathbb{C}^{2}}\right\} d y .
\end{aligned}
$$


Since $\left|\alpha_{1}+\alpha_{2}\right|<2$, it follows that the matrix

$$
R_{1}+R_{1}^{*}=\left(\begin{array}{cc}
2 & \alpha_{1}+\alpha_{2} \\
\alpha_{1}+\alpha_{2} & 2
\end{array}\right)
$$

is positive definite. Using the Silvester criterion, we will show that the matrix

$$
e^{i h} R_{1}+\left(e^{i h} R_{1}\right)^{*}=\left(\begin{array}{cc}
e^{i h}+e^{-i h} & e^{i h} \alpha_{1}+e^{-i h} \alpha_{2} \\
e^{i h} \alpha_{2}+e^{-i h} \alpha_{1} & e^{i h}+e^{-i h}
\end{array}\right)
$$

is also positive definite. Since $-\pi / 2<h<\pi / 2$, it follows that $e^{i h}+e^{-i h}>0$. Thus, we have to prove that $\operatorname{det}\left(e^{i h} R_{1}+\left(e^{i h} R_{1}\right)^{*}\right)>0$. Let $e^{i h}=\mu+i \nu$, where $\mu>0$. Using the equality $\nu^{2}=1-\mu^{2}$, we obtain

$$
\operatorname{det}\left(e^{i h} R_{1}+\left(e^{i h} R_{1}\right)^{*}\right)=4 \mu^{2}-\left[\left(\alpha_{1}+\alpha_{2}\right)^{2}+\left(4 \mu^{2}-4\right) \alpha_{1} \alpha_{2}\right] .
$$

Since $\left|\alpha_{1}+\alpha_{2}\right|<2$, it follows that, for $h=0$ (i.e., $\mu=1$ ),

$$
\operatorname{det}\left(e^{i h} R_{1}+\left(e^{i h} R_{1}\right)^{*}\right)=4-\left(\alpha_{1}+\alpha_{2}\right)^{2}>0 .
$$

Therefore, there is a number $h_{1}=h_{1}\left(\alpha_{1}, \alpha_{2}\right)>0$ such that

$$
\operatorname{det}\left(e^{i h} R_{1}+\left(e^{i h} R_{1}\right)^{*}\right)>0, \quad|h| \leq h_{1} .
$$

It follows from (8.19), from the positive definiteness of the matrices (8.20) and (8.21), and from the relation $\omega_{1}^{2}+\omega_{2}^{2}=1$ that

$$
\operatorname{Re} b_{\mathcal{R}}[u, u] \geq k_{2} \int_{\theta_{1}}\left\{\sum_{i}\left(\mathcal{U} u_{y_{i}}, \mathcal{U} u_{y_{i}}\right)_{\mathbb{C}^{2}}+(\mathcal{U} u, \mathcal{U} u)_{\mathbb{C}^{2}}\right\} d y=k_{2}\|u\|_{W^{1}(\theta)}^{2},
$$

where $k_{2}>0$ does not depend on $u$.

Inequalities (8.18) and (8.22) imply that $b_{\mathcal{R}}$ is a closed sectorial form on $L_{2}(\theta)$, with the domain $\mathrm{D}\left(b_{\mathcal{R}}\right)=\stackrel{\circ}{W}^{1}(\theta)$ and vertex $k_{2}>0$ (see [13, Chap. 6]).

Thus, repeating the reasoning of Example 2.1, we see that $\mathcal{L}(\omega), \omega \in S^{1}$, is an isomorphism for the above $a, \alpha_{1}, \alpha_{2}$, and $h$. Moreover, since $h$ and $\omega$ belong to compact sets, it follows that the inequality

$$
\|V\|_{E_{a}^{2}(\theta)} \leq k_{3}\|\mathcal{L}(\omega) V\|_{\mathcal{E}_{a}^{0}(\theta, \gamma)}, \quad \omega \in S^{1},
$$

holds with a constant $k_{3}>0$ that does not depend 4 on $V, \omega$, or $h$.

Now Theorem 8.1 implies that the operator $\mathcal{L}(p)$ is also an isomorphism for $p \geq p_{0}$, where $p_{0}>0$. Moreover, it follows from (8.23) that the inequality

$$
\|u\|_{H_{a}^{2}(\Theta)} \leq k_{4}\|\mathcal{L}(p) u\|_{\mathcal{H}_{a}^{0}(\Theta, \Gamma)}, \quad p>p_{0},
$$

holds with a constant $k_{4}>0$ that does not depend on $u$ or $h$.

3. Similarly to (6.17) and (6.18), using Lemma 7.1) estimate (7.15), and the interpolation inequalities in Sobolev spaces (see [2, Chap. 1, Sec. 1]), we obtain

$$
\begin{aligned}
& \left\|B_{l}^{2} u\right\|_{H_{a}^{3 / 2}\left(\Gamma_{l}\right)} \leq k_{5}\|u\|_{H_{a}^{2}\left(Q \backslash \overline{\mathcal{K}_{1}^{2 \varkappa}}\right)}, \\
& \left\|B_{l}^{2} u\right\|_{H_{a}^{3 / 2}\left(\Gamma_{l} \backslash \overline{\mathcal{K}_{1}^{\varkappa}}\right)} \leq k_{6}\|u\|_{H_{a}^{2}\left(Q_{\varkappa}\right)},
\end{aligned}
$$

where $a>1$ and $k_{5}, k_{6}>0$ do not depend on $u$. Thus, the operators $B_{l}^{2}$ satisfy Condition 7.4 for $a>1$.

We consider the linear bounded operators

$$
\mathbf{L}(p), \mathbf{L}^{1}(p): H_{a}^{2}(Q) \rightarrow H_{a}^{0}(Q) \times H_{a}^{3 / 2}\left(\Gamma_{1}\right) \times H_{a}^{3 / 2}\left(\Gamma_{2}\right)
$$

\footnotetext{
${ }^{4}$ Otherwise, denoting $L_{h}(\omega)=L(\omega)$, we see that there are sequences $h^{(k)}, \omega^{(k)}$, and $V^{(k)}, k=$ $1,2, \ldots$, such that $h^{(k)} \rightarrow h, \omega^{(k)} \rightarrow \omega,\left\|\mathcal{L}_{h^{(k)}}\left(\omega^{(k)}\right) V^{(k)}\right\|_{\mathcal{E}_{a}^{0}(\theta, \gamma)} \rightarrow 0$, and $\left\|V^{(k)}\right\|_{E_{a}^{2}(\theta)}=1$. This leads to a contradiction since $1=\left\|V^{(k)}\right\|_{E_{a}^{2}(\theta)} \leq c\left\|\mathcal{L}_{h}(\omega) V^{(k)}\right\|_{\mathcal{E}_{a}^{0}(\theta, \gamma)} \leq c\left(\left\|\mathcal{L}_{h^{(k)}}\left(\omega^{(k)}\right) V^{(k)}\right\|_{\mathcal{E}_{a}^{0}(\theta, \gamma)}+\right.$ $\left.\left\|\left(\mathcal{L}_{h^{(k)}}\left(\omega^{(k)}\right) V^{(k)}-\mathcal{L}_{h}(\omega) V^{(k)}\right)\right\|_{\mathcal{E}_{a}^{0}(\theta, \gamma)}\right) \stackrel{a}{\rightarrow}$.
} 
given by

$$
\begin{aligned}
\mathbf{L}(p) u & =\left\{-\Delta u+e^{i h} p^{2} u,\left.u\right|_{\Gamma_{l}}+B_{l}^{1} u+B_{l}^{2} u\right\}, \\
\mathbf{L}^{1}(p) u & =\left\{-\Delta u+e^{i h} p^{2} u,\left.u\right|_{\Gamma_{l}}+B_{l}^{1} u\right\} .
\end{aligned}
$$

The two results below follow from Lemma 8.2 and Theorem 8.1

Corollary 8.1. Let the numbers a, $\alpha_{1}, \alpha_{2}$ satisfy relations (8.17). Then there exist a number $h_{1}=h_{1}\left(\alpha_{1}, \alpha_{2}\right)>0$ and a number $p_{0}>0$, independent of $h$, such that the operator $\mathbf{L}^{1}(p), p \geq p_{0},|h| \leq h_{1}$, has a bounded inverse and

$$
c_{1}\left\|\mathbf{L}^{1}(p) u\right\|_{\mathcal{H}_{a}^{0}(Q, \Gamma)} \leq\|u\|_{H_{a}^{2}(Q)} \leq c_{2}\left\|\mathbf{L}^{1}(p) u\right\|_{\mathcal{H}_{a}^{0}(Q, \Gamma)},
$$

where $c_{1}, c_{2}>0$ do not depend on $u$, $h$, or $p$.

Corollary 8.2. Let $1<a \leq 2$, while the numbers $\alpha_{1}, \alpha_{2}$, and $h_{1}$ are the same as in Corollary 8.1. Then there is a number $p_{1}>0$, independent of $h$, such that the operator $\mathbf{L}(p), p \geq p_{1},|h| \leq h_{1}$, has a bounded inverse and

$$
c_{1}\|\mathbf{L}(p) u\|_{\mathcal{H}_{a}^{0}(Q, \Gamma)} \leq\|u\|_{H_{a}^{2}(Q)} \leq c_{2}\|\mathbf{L}(p) u\|_{\mathcal{H}_{a}^{0}(Q, \Gamma)},
$$

where $c_{1}, c_{2}>0$ do not depend on $u, h$, or $p$.

Remark 8.1. Let $\alpha_{1}=\alpha_{2}$ and $\left|\alpha_{1}\right|<1$. In this case, Corollaries 8.1 and 8.2 are true for any $h_{1}$ satisfying the relation $0<h_{1}<\pi / 2$. Indeed, the matrix (8.21) remains positive definite because

$$
e^{i h}+e^{-i h}=2 \mu>0, \quad \operatorname{det}\left(e^{i h} R_{1}+\left(e^{i h} R_{1}\right)^{*}\right)=4 \mu^{2}\left(1-\alpha_{1}^{2}\right)>0,
$$

where $\mu=\operatorname{Re} e^{i h}>0$. Therefore, the form $b_{\mathcal{R}}$ is again a closed sectorial form on $L_{2}(\theta)$ with the domain $\mathrm{D}\left(b_{\mathcal{R}}\right)=\stackrel{\circ}{W}^{1}(\theta)$ and vertex $k_{2}>0$. Further argument does not change.

4. Consider the unbounded operators $\mathbf{A}, \mathbf{A}^{1}: H_{a}^{0}(Q) \rightarrow H_{a}^{0}(Q)$ given by

$$
\begin{aligned}
\mathbf{A} u & =-\Delta u, & u & \in \mathrm{D}(\mathbf{A})=\left\{u \in H_{a}^{2}(Q):\left.u\right|_{\Gamma_{l}}+B_{l}^{1} u+B_{l}^{2} u=0\right\}, \\
\mathbf{A}^{1} u & =-\Delta u, & u & \in \mathrm{D}(\mathbf{A})=\left\{u \in H_{a}^{2}(Q):\left.u\right|_{\Gamma_{l}}+B_{l}^{1} u=0\right\} .
\end{aligned}
$$

Corollary 8.1 implies the following result (see Figure 5).

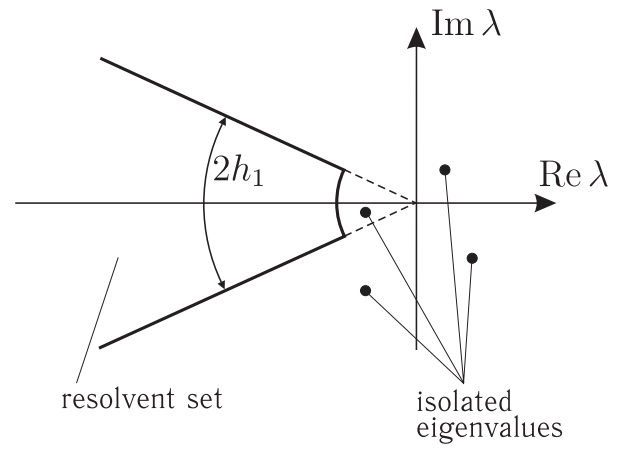

Figure 5. Spectra of the operators $\mathbf{A}^{1}$ and $\mathbf{A}$

Corollary 8.3. Let the conditions of Corollary 8.1 hold. Then the following assertions are true:

(1) the spectrum $\sigma\left(\mathbf{A}^{1}\right)$ is discrete 5

\footnotetext{
${ }^{5}$ This means that the spectrum consists of a finite or a countable set of isolated eigenvalues of finite multiplicity.
} 
(2) there exist numbers $h_{1}=h_{1}\left(\alpha_{1}, \alpha_{2}\right)>0$ and $\lambda_{1}>0$ such that $\sigma\left(\mathbf{A}^{1}\right) \subset \mathbb{C} \backslash \Omega_{h_{1}, \lambda_{1}}$, where

$$
\Omega_{h_{1}, \lambda_{1}}=\left\{\lambda \in \mathbb{C}:|\arg (\lambda-\pi)| \leq h_{1},|\lambda| \geq \lambda_{1}\right\}
$$

(3) the estimate

$$
\left\|\left(\mathbf{A}^{1}-\lambda \mathbf{I}\right)^{-1}\right\|_{H_{a}^{0}(Q) \rightarrow H_{a}^{0}(Q)} \leq c_{1} /|\lambda|, \quad \lambda \in \Omega_{h_{1}, \lambda_{1}}
$$

holds with a constant $c_{1}=c_{1}\left(h_{1}\right)>0$.

Proof. Set $-\lambda=e^{i h} p^{2}$ and $\lambda_{1}=p_{0}^{2}$, where $p_{0}$ is the constant occurring in Corollary 8.1, In this case, assertions 2 and 3 follow from Corollary 8.1. By the same corollary, the operator $(\mathbf{A}-\lambda \mathbf{I})^{-1}: H_{a}^{0}(Q) \rightarrow H_{a}^{2}(Q)$ is bounded for $\lambda \in \Omega_{h_{1}, \lambda_{1}}$. Combining this fact with the compactness of the embedding $H_{a}^{2}(Q) \subset H_{a}^{0}(Q)$, we see that the resolvent $(\mathbf{A}-\lambda \mathbf{I})^{-1}: H_{a}^{0}(Q) \rightarrow H_{a}^{0}(Q)$ is compact for $\lambda \in \Omega_{h_{1}, \lambda_{1}}$. Therefore, by Theorem 6.29 in [13, Chap. 3, Sec. 6], assertion 1 is true.

Using Corollary 8.2 instead of Corollary 8.1, we obtain the following result (see Figure 5).

Corollary 8.4. Let the conditions of Corollary 8.2 hold. Then the following assertions are true:

(1) the spectrum $\sigma(\mathbf{A})$ is discrete;

(2) there exists a number $\lambda_{2}>0$ such that $\sigma(\mathbf{A}) \subset \mathbb{C} \backslash \Omega_{h_{1}, \lambda_{2}}$;

(3) the estimate

$$
\left\|(\mathbf{A}-\lambda \mathbf{I})^{-1}\right\|_{H_{a}^{0}(Q) \rightarrow H_{a}^{0}(Q)} \leq c_{2} /|\lambda|, \quad \lambda \in \Omega_{h_{1}, \lambda_{2}},
$$

holds with a constant $c_{2}=c_{2}\left(h_{1}\right)>0$, where $h_{1}>0$ is the constant from Corollary 8.3 .

Remark 8.2. Let $\alpha_{1}=\alpha_{2}$ and $\left|\alpha_{1}\right|<1$. In this case, Corollaries 8.3 and 8.4 are true for any $h_{1}$ satisfying the relation $0<h_{1}<\pi / 2$ (cf. Remark 8.1).

The following questions are unanswered. Do there exist a number $h_{1}, \pi / 2 \leq h_{1}<\pi$, and numbers $\lambda_{1}, \lambda_{2}>0$ such that

$$
\sigma\left(\mathbf{A}^{1}\right) \subset \mathbb{C} \backslash \Omega_{h_{1}, \lambda_{1}}, \quad \sigma(\mathbf{A}) \subset \mathbb{C} \backslash \Omega_{h_{1}, \lambda_{2}} ?
$$

Can one find, for any $h_{1}, 0<h_{1}<\pi$, numbers $\lambda_{1}, \lambda_{2}>0$ such that relations (8.25) hold (cf. Problem 13.1 in [28, Sec. 13])?

\section{REFERENCES}

[1] S. Agmon, A. Douglis, L. Nirenberg, "Estimates near the boundary for solutions of elliptic partial differential equations satisfying general boundary conditions. I," Comm. Pure Appl. Math., 12, 623-727 (1959). MR0125307(23:A2610)

[2] M. S. Agranovich and M. I. Vishik, "Elliptic problems with a parameter and parabolic problems of general type," Uspekhi Mat. Nauk, 19, 53-161 (1964); English transl.: Russian Math. Surveys, 19 (1964). MR0192188 (33:415)

[3] A. V. Bitsadze, A. A. Samarskiř, "On some simple generalizations of linear elliptic boundary value problems," Dokl. Akad. Nauk SSSR., 185, 739-740 (1969); English transl.: Soviet Math. Dokl., 10 (1969). MR0247271(40:540)

[4] I. C. Gohberg and E. I. Sigal, "An operator generalization of the logarithmic residue theorem and the theorem of Rouché," Mat. Sb., 84 (126), 607-629 (1971); English transl.: Math. USSR Sb., 13, 603-625 (1971). MR0313856 (47:2409)

[5] P. L. Gurevich, "Nonlocal problems for elliptic equations in dihedral angles and the Green formula," Mitteilungen aus dem Mathem. Seminar Giessen, Math. Inst. Univ. Giessen, Germany, 247, 1-74 (2001). MR 1867955 (2003a:35047) 
[6] P. L. Gurevich, "Asymptotics of solutions for nonlocal elliptic problems in plane angles," Trudy Seminara imeni I. G. Petrovskogo, 23, 93-126 (2003); English transl.: J. Math. Sci., 120, No. 3, 1295-1312 (2004). MR2085182(2005e:35049)

[7] P. L. Gurevich, "Non-local elliptic problems with non-linear argument transformations near the points of conjugation," Izv. Ross. Akad. Nauk. Ser. Mat., 67, No. 6, 71-110 (2003); English transl.: Izv. Math., 67, No. 6, 1149-1186 (2003). MR2032091(2005j:35037)

[8] P. L. Gurevich, "Solvability of nonlocal elliptic problems in Sobolev spaces, I" Russ. J. Math. Phys., 10, No. 4, 436-466 (2003). MR2096605 (2005g:35062)

[9] P. L. Gurevich, "Solvability of nonlocal elliptic problems in Sobolev spaces, II" Russ. J. Math. Phys., 11, No. 1, 1-44 (2004). MR2134647(2005k:35086)

[10] P. L. Gurevich, "Smoothness of Generalized Solutions to Nonlocal Elliptic Problems on the Plane" Dokl. Akad. Nauk, 398, No. 3, 295-299 (2004); English transl.: Russian Acad. Sci. Dokl. Math., 70, 714-718 (2004). MR2116507 (2005k:35080)

[11] A. K. Gushchin, "A condition for the compactness of operators in a certain class and its application to the analysis of the solubility of non-local problems for elliptic equations," Mat. Sb., 193, No. 5, 17-36 (2002); English transl.: Sb. Math., 193, No. 5, 649-668 (2002). MR.1918245 (2004c:35147)

[12] A. K. Gushchin, V. P. Mikhailov, "On solvability of nonlocal problems for elliptic equations of second order," Mat. Sb., 185, 121-160 (1994); English transl.: Russian Acad. Sci. Sb. Math., 81, 101-136 (1995). MR1264079 (94m:35088)

[13] T. Kato, Perturbation Theory for Linear Operators, Springer-Verlag, Berlin-Heidelberg-New York, 1966. MR0203473(34:3324)

[14] K. Yu. Kishkis, "The index of a Bitsadze-Samarskii Problem for harmonic functions," Differentsial'nye Uravneniya., 24, No. 1, 105-110 (1988); English transl.: Diff. Equ., 24, 83-87 (1988). MR.930160 (89h:35092)

[15] V. A. Kondrat'ev, "Boundary value problems for elliptic equations in domains with conical or angular points," Trudy Moskov. Mat. Obshch., 16, 209-292 (1967); English transl.: Trans. Moscow Math. Soc., 16 (1967). MR0226187 (37:1777)

[16] V. A. Kondrat'ev and O. A. Oleinik, "Boundary value problems for partial differential equations in non-smooth domains," Uspekhi Mat. Nauk., 38, 3-75 (1983); English transl.: Russian Math. Surveys, 38, 1-86 (1983). MR695471 (85j:35002)

[17] O. A. Kovaleva and A. L. Skubachevskii, "Solvability of nonlocal elliptic problems in weighted spaces," Mat. Zametki., 67, 882-898 (2000); English transl.: Math. Notes., 67 (2000). MR1820643 (2002j:35089)

[18] S. G. Krein, Linear Equations in a Banach Space, Nauka, Moscow, 1971; English transl.: Birkhäuser, Boston, 1982. MR0374949 (51:11145)

[19] J.-L. Lions and E. Magenes, Non-Homogeneous Boundary Value Problems and Applications, Vol. 1, Springer-Verlag, New York-Heidelberg-Berlin, 1972. MR0350177 (50:2670)

[20] V. G. Maz'ya and B. A. Plamenevskii, " $L_{p}$-estimates of solutions of elliptic boundary value problems in domains with edges," Trudy Moskov. Mat. Obshch., 37, 49-93 (1978); English transl.: Trans. Moscow Math. Soc., 37 (1980). MR514327 (81b:35027)

[21] C. Miranda, Equazioni Alle Derivate Parziali di Tipo Ellittico, Springer-Verlag, Berlin-GöttingenHiedelberg, 1955. MR0087853 (19:421d)

[22] S. A. Nazarov and B. A. Plamenevskii, Elliptic Problems in Domains with Piecewise Smooth Boundaries, Moscow: Nauka, 1991; English transl. in De Gruyter Expositions in Mathematics, 13. Walter de Gruyter Publishers, Berlin-New York, 1994. MR1283387 (95h:35001)

[23] M. A. Skryabin, "Nonlocal Elliptic Problems in Dihedral Angles and Functional Differential Equations," Sovremennaya Matematika. Fundamental'nye Napravleniya, 4, 121-143 (2003); English transl.: J. Math. Sci., 129, No. 5, 4227-4249 (2005). MR2122347(2005j:35075)

[24] A. L. Skubachevskii, "Elliptic problems with nonlocal conditions near the boundary," Mat. Sb., 129 (171), 279-302 (1986); English transl.: Math. USSR Sb., 57, 279-302 (1987). MR832122 (87h:35089)

[25] A. L. Skubachevskii, "Model nonlocal problems for elliptic equations in dihedral angles," Differentsial'nye Uravneniya, 26, 119-131 (1990); English transl.: Differ. Equ., 26, 106-115 (1990). MR $1050367(91 \mathrm{~h}: 35119)$

[26] A. L. Skubachevskii, "Truncation-function method in the theory of nonlocal problems," Differentsial'nye Uravneniya, 27, 128-139 (1991); English transl.: Diff. Equ., 27, 103-112 (1991). MR:1133518 (92k:35055)

[27] A. L. Skubachevskii, "On the stability of index of nonlocal elliptic problems," J. of Mathematical Analysis and Applications, 160, 323-341 (1991). MR1126121(92m:58128) 
[28] A. L. Skubachevskii, Elliptic Functional Differential Equations and Applications, Basel-BostonBerlin, Birkhäuser, 1997. MR:1437607 (98c:35164)

[29] A. L. Skubachevskii, "Regularity of solutions of a nonlocal elliptic problem," Russ. J. Math. Phys., 8, 365-374 (2001). MR1930382 (2003g:35044)

[30] L. N. Slobodetskii, "Generalized Sobolev spaces and their application to boundary problems for partial differential equations," Leningrad. Gos. Ped. Inst. Uchen.Zap., 197, 54-112 (1958); English transl.: Amer. Math. Soc. Transl. Ser. 2, 57, 207-275 (1966). MR0203222 (34:3075)

[31] L. R. Volevich "Solvability of boundary-value problems for general elliptic systems," Mat. Sb., 68, No. 3, 373-416 (1965). MR0192191 (33:418)

Department of Differential Equations and Mathematical Physics, Peoples' Friendship University of Russia, Miklukho-Maklaya St. 6, 117198 Moscow, Russia

Current address: Interdisciplinary Center for Scientific Computing of the University of Heidelberg Im Neuenheimer Feld 368, D-69120 Heidelberg, Germany

E-mail address: pavel.gurevich@iwr.uni-heidelberg.de

Department of Differential Equations and Mathematical Physics, Peoples' Friendship UniVersity of Russia, Miklukho-Maklaya St. 6, 117198 Moscow, Russia

E-mail address: skub@lector.ru

Translated by THE AUTHORS 